\title{
Reach Control on Simplices By Piecewise Affine Feedback
}

by

\author{
Marcus Ganness
}

A thesis submitted in conformity with the requirements for the degree of Master of Applied Science Graduate Department of Electrical and Computer Engineering University of Toronto

Copyright (C) 2010 by Marcus Ganness 


\begin{abstract}
Reach Control on Simplices By Piecewise Affine Feedback

Marcus Ganness

Master of Applied Science

Graduate Department of Electrical and Computer Engineering

University of Toronto

2010
\end{abstract}

This thesis provides a deep study of the Reach Control Problem (RCP) for affine systems defined on simplices. Necessary conditions for solvability of the problem by open loop control are presented, improving upon the results in the literature which are for continuous state feedback only. So-called reach control indices are introduced and developed which inform on the structural properties of the system which cause continuous state feedbacks to fail. A novel synthesis method is presented consisting of a subdivision algorithm based on these indices and an associated piecewise affine feedback. The method is shown to solve RCP for all cases in the literature where continuous state feedback fails, provided it is solvable by open loop control. Textbook examples of existing synthesis methods for RCP are provided. The motivation for studying RCP and its relevance to complex control specifications is illustrated using a biomedical application. 


\section{Dedication}

To God, the creator of all things, and to all students throughout the world who were not as fortunate to have received the great learning opportunity that I have been blessed with. 


\section{Acknowledgements}

First and foremost I must thank my supervisor Professor Mireille Broucke, for allowing me to be part of the exciting and groundbreaking research in which she is undertaking at the University of Toronto. Also, for her clarity, patience and commitment to making this thesis possible I am extremely grateful. To my parents and my family who have supported my during the past two years, I am also indebted. Finally I would like to thank all the staff and students within the Systems Control Group for making this experience an unforgettable and overall enjoyable learning experience. 


\section{Contents}

1 Introduction $\quad 1$

1.1 Bibliographic Notes . . . . . . . . . . . . . . . . 3

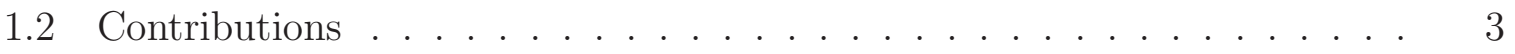

2 Background $\quad 5$

2.1 Notation ............................. 5

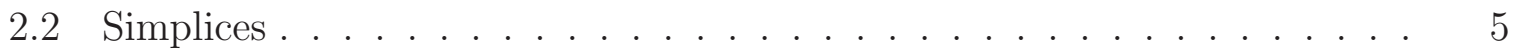

2.3 Placing Triangulation . . . . . . . . . . . . . . . . 8

$2.4 \mathscr{M}$-Matrices . . . . . . . . . . . . . . . . . . . . 9

2.5 Convex Analysis . . . . . . . . . . . . . . . . . . . . . . 10

2.6 Nonsmooth Analysis . . . . . . . . . . . . . . . . . . . . . 11

2.7 Fixed Point Theory . . . . . . . . . . . . . . . . . . . 12

2.7 .1 Sperner's Lemma . . . . . . . . . . . . . . 12

3 Reach Control: Basic Principles 14

3.1 Reach Control Problem . . . . . . . . . . . . . . . . . 14

3.2 Necessary Conditions . . . . . . . . . . . . . . . . . . 17

3.3 Affine Systems . . . . . . . . . . . . . . . . . . . 22

3.4 Affine Feedback . . . . . . . . . . . . . . . . . . . . 26

4 Triangulations and Affine Feedback $\quad 29$ 
4.1 Motivation . . . . . . . . . . . . . . . . . . . . . . . . . . . . . . . 29

4.2 Affine Feedback . . . . . . . . . . . . . . . . . . 30

$5 \quad$ Reach Control and Continuous State Feedback $\quad 37$

5.1 Preliminaries . . . . . . . . . . . . . . . . . . . . . . . . 38

5.2 Affine Feedback Transformations _ . . . . . . . . . . . . . . . . . . 39

5.3 Reach Control Indices _. . . . . . . . . . . . . . . . . . . . . . . 42

5.4 Existence of Equilibria . . . . . . . . . . . . . . . . . . 49

5.5 Continuous State Feedback . . . . . . . . . . . . . . . . . 53

6 Reach Control and Piecewise Affine Feedback 55

6.1 RCP Revisited . . . . . . . . . . . . . . . . . . 55

6.2 Piecewise Affine Feedback . . . . . . . . . . . . . . . . 56

6.3 Main Result . . . . . . . . . . . . . . . . . . . . . . 66

$\begin{array}{lll}7 & \text { Examples } & 67\end{array}$

7.1 RCP solution using affine feedback $: \mathcal{G}=\emptyset \ldots \ldots \ldots 7$

7.1 .1 Example $1 \ldots \ldots \ldots \ldots \ldots$

7.1 .2 Example $2 \ldots \ldots \ldots \ldots \ldots \ldots \ldots$

7.2 RCP solution using affine feedback: $\mathcal{G} \neq \emptyset$ and $\mathcal{B} \cap \operatorname{cone}(\mathcal{S}) \neq \mathbf{0} \ldots 70$

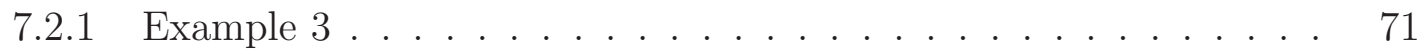

7.3 RCP solution using piecewise affine feedback . . . . . . . . . . 73

7.3 .1 Example $4 \ldots \ldots \ldots \ldots \ldots \ldots \ldots \ldots$

7.3 .2 Example $5 \ldots \ldots \ldots \ldots \ldots \ldots \ldots \ldots$

7.3 .3 Example $6 \ldots \ldots \ldots \ldots \ldots \ldots \ldots \ldots$

7.3 .4 Example $7 \ldots \ldots \ldots \ldots \ldots \ldots \ldots \ldots$

7.3 .5 Example $8 \ldots \ldots \ldots \ldots \ldots \ldots \ldots \ldots$

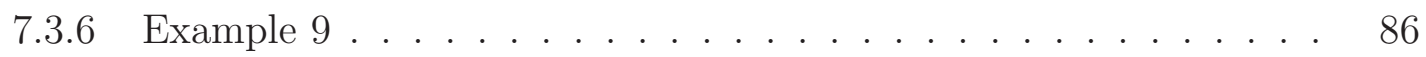

7.3 .7 Example $10 \ldots \ldots \ldots \ldots \ldots$ 
7.4 Further ideas on RCP solution . . . . . . . . . . . . . . . . . . . . 91

7.4 .1 Example $11 \ldots \ldots \ldots \ldots 2 \ldots \ldots \ldots \ldots$

7.4 .2 Example $12 \ldots \ldots \ldots \ldots$

8 A Biomedical Application $\quad 96$

8.1 Anesthesia Delivery and Control . . . . . . . . . . . . . . . . . 96

8.2 A Full Model For BIS Control . . . . . . . . . . . . . . . . . . . . . . . 100

8.3 Case 1: A Simplified model in $\mathbb{R}^{2} \ldots \ldots$. . . . . . . . . . . 104

8.3.1 Complex Control Specifications . . . . . . . . . . . . 106

8.3 .2 Control Implementation . . . . . . . . . . . . . . . . 109

8.4 Case 2: Simplified model in $\mathbb{R}^{3} \ldots \ldots \ldots \ldots$

8.4.1 Complex Control Specifications _. . . . . . . . . . . . . 119

8.4 .2 Control Implementation _. . . . . . . . . . . . . . . 122

9 Conclusions 132

$\begin{array}{ll}\text { Bibliography } & 134\end{array}$ 


\section{List of Figures}

2.1 Example of Sperner's Lemma _ . . . . . . . . . . . . . . . . . . . 13

3.1 Geometric constructs for the reach control problem. . . . . . . . . . 18

4.1 Continuous Assignment Along $\mathcal{G}$ of Vectors in $\mathcal{B} . \ldots \ldots 33$

4.2 Not enough degrees of freedom in $\mathcal{B} \ldots \ldots \ldots$

6.1 Subdivision into two simplices $\mathcal{S}^{\prime}$ and $\mathcal{S}_{1} \ldots \ldots \ldots \ldots$

7.1 Example 1: Closed loop vector field on $\mathcal{S} \ldots \ldots \ldots$

7.2 Example 2: Closed loop vector field on $\mathcal{S}$. . . . . . . . . . . 71

7.3 Example 3: Closed loop vector field on $\mathcal{S} \ldots \ldots \ldots$

7.4 Example 4: Closed loop vector field on $\mathcal{S} \ldots \ldots \ldots \ldots$

7.5 Example 4: Closed loop vector field on $\mathcal{S}_{2} \ldots \ldots \ldots \ldots$

7.6 Example 4: Closed loop vector field on $\mathcal{S}_{1} \ldots \ldots \ldots \ldots$

7.7 Example 4: Closed loop vector field on $\mathcal{S}$ under piecewise affine feedback 76

7.8 Example 5: Closed loop vector field on $\mathcal{S}_{2} \ldots \ldots \ldots$

7.9 Example 5: Closed loop vector field on $\mathcal{S}_{1} \ldots \ldots \ldots 78$

7.10 Example 5: Closed loop vector field on $\mathcal{S}$ under piecewise affine feedback 78

7.11 Example 6: Trajectories of initial conditions in $\mathcal{S}$ under piecewise affine feedback $($ view 1$) \ldots \ldots \ldots \ldots \ldots \ldots \ldots \ldots$

7.12 Example 6: Trajectories of initial conditions in $\mathcal{S}$ under piecewise affine

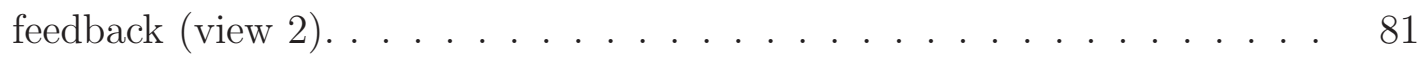


7.13 Example 6: Trajectories of initial conditions in $\mathcal{S}$ under piecewise affine feedback (view 3). . . . . . . . . . . . . . . . 82

7.14 Example 11: View 1 showing trajectories with initial conditions not in $\mathcal{G}$ all leave $\mathcal{S} \ldots \ldots \ldots$. . . . . . . . . . . . . . . . . . . . . . . . . . 93

7.15 Example 11: View 2 showing trajectories with initial conditions not in $\mathcal{G}$ all leave $\mathcal{S} \ldots \ldots \ldots \ldots \ldots$. . . . . . . . . . . . . . . . . . . . 94

7.16 Example 12: Closed loop vector field on $\mathcal{S} \ldots \ldots$. . . . . . . . . . . . 95

8.1 A three-compartment PK model . . . . . . . . . . . . . . . . . . . 98

8.2 Overall PK-PD model . . . . . . . . . . . . . . . . . . . . . . . . . . . 99

8.3 Schematic representation of breathing model as shown in [30] . . . . . . . 101

8.4 Five compartment mamilliary model . . . . . . . . . . . . . . . . . . 102

8.5 Diffusion Constants . . . . . . . . . . . . . . . . . . . 104

8.6 Compartment Volumes . . . . . . . . . . . . . . . . 104

8.7 BIS variation under various stages of anesthesia as reported in [33] . . . . 107

8.8 Discrete Automaton representing control specification: Case 1 . . . . . 110

8.9 BIS variation with central compartment concentration . . . . . . . . . 112

8.10 State-space areas satisfying constraints for modes/events of control speci-

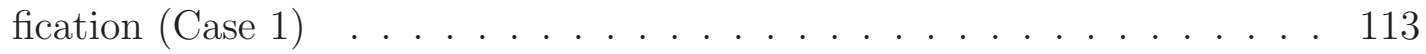

8.11 Triangulated state-space showing state trajectory under piecewise affine controller: Case 1 . . . . . . . . . . . . . . . . . 116

8.12 BIS variation under piecewise affine controller: Case 1 . . . . . . . . . 117

$8.13 x_{1}$ variation under piecewise affine controller: Case $1 \ldots \ldots$. . . . . . 117

$8.14 x_{2}$ variation under piecewise affine controller: Case $1 \ldots \ldots$. . . . 118

8.15 Discrete Automaton representing control specification: Case 2 . . . . . . 122

8.16 State-space areas satisfying constraints for modes/events of control specification $($ Case 2) . . . . . . . . . . . . . . . . . . . . . 125 
8.17 Triangulated state-space showing state trajectory under piecewise affine

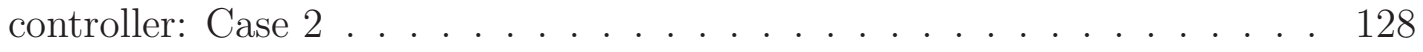

8.18 BIS variation under piecewise affine controller: Case 2 . . . . . . . . . . 129

8.19 Inspired drug concentration under piecewise affine controller: Case 2 . . . 129

8.20 Central compartment drug concentration under piecewise affine controller: Case $2 \ldots \ldots \ldots \ldots$. . . . . . . . . . . . . . . . . . . . . . 130

8.21 Second compartment drug concentration under piecewise affine controller: Case $2 \ldots \ldots \ldots \ldots 131$ 


\section{Chapter 1}

\section{Introduction}

Control theory has traditionally been focused on problems of stabilization and regulation, and design methods arising out of research on these problems have been extensively applied in industry. The increasingly complex requirements for modern engineering systems, however, have highlighted the need for the development of control techniques which achieve complex specifications. For instance, a control problem in an industrial or biomedical setting will typically have some steady state control objectives. In addition, specific requirements on the transient response may also be present for various reasons such as safety or efficiency. In essence, the system must be transferred from initial condition to steady state according to constraints requiring a high degree of expressivity in the specification terminology, requiring precise sequences of behaviors or repetitions of behaviors, and having qualitative temporal properties which are not easily encoded as a regulation-based or steady-state problem.

Piecewise affine feedback control has been proposed as a paradigm to achieve complex control specifications [15]. This paradigm involves the subdivision of the state space into simplices via a process of triangulation. With the control specification encoded as a discrete automaton and groups of simplices corresponding to nodes, a piecewise affine feedback can be applied so that the state moves through the sequence of simplices 
representative of the automaton behavior. The problem of ensuring that trajectories reach a facet of a simplex in finite time, known as the reach control problem $(\mathrm{RCP})$, is extremely relevant to such control designs.

This thesis presents a deep study of the reach control problem for an affine system defined on a simplex. The problem was first introduced in [14] and further developed in $[15,16,22,9]$. While we do not dwell upon the larger context of the problem, such explanations are well elaborated in the literature. Our focus, instead, is on developing synthesis methods to solve RCP in those cases where no solution is known. This thesis expands on previous work and, in particular, proposes a solution to RCP by piecewise affine feedback. The main outcome of the study is that piecewise affine feedback solves RCP in all known cases of the problem where the application of continuous state feedback fails.

The thesis is organized as follows. Chapter 2 introduces the necessary technical background including notation and important concepts relating to simplex geometry, convex analysis, non-smooth analysis, and fixed point theory. In Chapter 3, the reach control problem, the central problem studied in the thesis, is formulated. The main features of the problem are developed and a first set of necessary and sufficient conditions for a solution by affine feedback is presented. Chapter 4 examines the problem under specific assumptions on the triangulation of the state space. New necessary and sufficient conditions for solvability by affine feedback are presented. These improve on the previous conditions since they are verifiable geometric properties of the system together with the simplex. Chapter 5 shows the equivalence of continuous state feedback and affine feedback from the point of view of solvability of RCP. So called reach control indices are introduced and developed in order to expose the structural properties of the system which cause continuous state feedbacks to fail. It is seen that the failure is manifested as a closed-loop equilibrium inside the simplex. Chapter 6 presents a subdivision algorithm based on the reach control indices for obtaining a piecewise affine feedback solution to 
RCP. We also present the main result of the thesis, which states that if RCP is solvable by open-loop controls, then it is solvable by piecewise affine feedback. Chapter 7 gives textbook examples of RCP and documents the implementation of the synthesis methods corresponding to the theory developed in the previous chapters. Chapter 8 illustrates the general idea of complex specifications via a biomedical application. Chapter 9 summarizes the main results of the thesis.

\subsection{Bibliographic Notes}

Chapter 2 includes review material drawn from the following sources. Properties of simplices can be found in several references on polytopes such as [27]. The placing triangulation is discussed in [18]. Extensive background on $\mathscr{M}$-matrices can be found in [4]. The material on non-smooth analysis is drawn from [11]. The material on fixed point theory can be found in several sources; see for instance [8] or [23]. The results of Chapter 3 represent the early results on RCP and are found in [15, 16, 22]. However, the section on necessary conditions for solvability is new to the thesis. Chapter 4 is largely drawn from [9]. Chapters 5-8 represent the original contributions of the thesis. Chapter 8 utilizes a model of anesthesia which is adapted from [30].

\subsection{Contributions}

We summarize the novel contributions of the thesis. In previous work [9] it was shown that if RCP is solvable by continuous state feedback, then it is solvable by affine feedback. This result was obtained by a geometric analysis which revealed cases when the problem was not solvable by continuous state feedback, yet the necessary conditions for solvability by open-loop control are satisfied. This suggested a need to study new control strategies beyond the class of continuous state feedback, and it is this gap which the thesis fills. 
- Chapter 3 includes necessary conditions for solvability of RCP by open-loop control. These results improve the results in the literature [15] which are for continuous state feedback only.

- In Chapter 5 we introduce so-called reach control indices. These indices provide geometric, structural information about the relationship between the control system and the simplex. This information, not suprizingly, arises in the form of certain rank conditions. Alternatively, the indices catalog parts of the simplex which are starved of sufficient inputs. It is shown that using the reach control indices, one can isolate closed-loop equilibria in those input-starved sub-simplices, whenever appropriate continuous state feedbacks are applied.

- In Chapter 6 we present a novel synthesis method that consists of a subdivision of the simplex and an associated piecewise affine feedback, with the property that any rank deficiency is "broken up" among several sub-simplices. We conclude that $\mathrm{RCP}$ is solvable by piecewise affine feedback whenever it is solvable by open-loop control.

- We present textbook examples of currently available synthesis methods for RCP in Chapter 7 .

- A biomedical application illustrating the general ideas underlying the research area is presented. It is intended to evoke further explorations of the possible applications of the theory. 


\section{Chapter 2}

\section{Background}

\section{$2.1 \quad$ Notation}

Let $\mathcal{K} \subset \mathbb{R}^{n}$ be a set. The complement of $\mathcal{K}$ is $\mathcal{K}^{c}:=\mathbb{R}^{n} \backslash \mathcal{K}$, the closure is $\overline{\mathcal{K}}$, and the interior is $\mathcal{K}^{\circ}$. For a vector $x \in \mathbb{R}^{n}$, the notation $x \succ 0(x \succeq 0)$ means $x_{i}>0\left(x_{i} \geq 0\right)$ for $1 \leq i \leq n$. The notation $x \prec 0(x \preceq 0)$ means $-x \succ 0(-x \succeq 0)$. For a matrix $A \in \mathbb{R}^{n \times n}$, the notation $A \succ 0(A \succeq 0)$ means $a_{i j}>0\left(a_{i j} \geq 0\right)$ for $1 \leq i, j \leq n$. Notation 0 denotes the subset of $\mathbb{R}^{n}$ containing only the zero vector. Notation $\mathscr{B}$ denotes the open unit ball, and $\overline{\mathscr{B}}$ denotes its closure. Notation co $\left\{v_{1}, v_{2}, \ldots\right\}$ denotes the convex hull of a set of points $v_{i} \in \mathbb{R}^{n}$. Notation aff $\left\{v_{1}, v_{2}, \ldots\right\}$ denotes the affine hull of a set of points $v_{i} \in \mathbb{R}^{n}$. Finally, $T_{\mathcal{S}}(x)$ denotes the Bouligand tangent cone to set $\mathcal{S}$ at a point $x$.

\subsection{Simplices}

The standard $n$-simplex is the subset of $\mathbb{R}^{n+1}$ given by

$$
\Delta^{n}:=\left\{\left(\alpha_{0}, \ldots, \alpha_{n}\right) \in \mathbb{R}^{n+1} \mid \sum_{i} \alpha_{i}=1, \alpha_{i} \geq 0\right\}
$$


More generally, an $n$-dimensional simplex is an $n$-dimensional polytope with $n+1$ vertices. Let

$$
V:=\left\{v_{0}, v_{1}, \ldots, v_{n}\right\}
$$

be a set of $n+1$ points in $\mathbb{R}^{n}$. We say $\left\{v_{0}, \ldots, v_{n}\right\}$ are affinely independent if they do not lie in an $(n-1)$-dimensional plane in $\mathbb{R}^{n}$. Equivalently, $\left\{v_{0}, \ldots, v_{n}\right\}$ are affinely independent if $\left\{v_{1}-v_{0}, \ldots, v_{n}-v_{0}\right\}$ are linearly independent. This provides another way to define a simplex: an $n$-dimensional simplex is the convex hull of $n+1$ affinely independent points in $\mathbb{R}^{n}$. The affine hull of $V$, denoted aff $\{V\}$, is the smallest affine space containing $V$.

Suppose that $V$ is affinely independent and define the simplex

$$
\mathcal{S}:=\operatorname{co}\left\{v_{0}, v_{1}, \ldots, v_{n}\right\}
$$

A face of $\mathcal{S}$ is any sub-simplex which makes up its boundary. An $(n-1)$-dimensional face of $\mathcal{S}$ is called a facet. We denote the facets of $\mathcal{S}$ by $\mathcal{F}_{0}, \ldots, \mathcal{F}_{n}$. Our numbering convention is such that

$$
\mathcal{F}_{i}=\operatorname{co}\left\{v \in V \backslash\left\{v_{i}\right\}\right\}, \quad i \in\{0,1, \ldots, n\}
$$

That is, each facet is indexed by the vertex it does not contain. Let $h_{i}$ denote the unit normal vector to $\mathcal{F}_{i}$, pointing out of $\mathcal{S}$. An implicit description of $\mathcal{S}$ is obtained using these normal vectors. Namely, there exist $\alpha_{0}, \ldots, \alpha_{n} \in \mathbb{R}$ such that

$$
\mathcal{S}=\left\{x \in \mathbb{R}^{n} \mid h_{i} \cdot x \leq \alpha_{i}, \quad \forall i \in\{0, \ldots, n\}\right\}
$$

Finally we collect some useful properties about simplices.

Lemma 2.1. Let $\mathcal{S}$ be a simplex. Then the following hold:

(i) If $x \in \operatorname{co}\left\{v_{1}, \ldots, v_{k}\right\}$, then $x \in \mathcal{F}_{j}$, for $k+1 \leq j \leq n$.

(ii) $h_{j} \cdot\left(v_{i}-v_{0}\right)=0$ for all $1 \leq i, j \leq n$ and $j \neq i$. 
(iii) $h_{j} \cdot\left(v_{i}-v_{k}\right)=0$ for all $0 \leq i, k \leq n$ and $j \neq i, k$.

(iv) $h_{i} \cdot\left(v_{i}-v_{0}\right)<0$, for all $1 \leq i \leq n$.

(v) $h_{j} \cdot\left(v_{i}-x\right)>0$ for all $x \in \mathcal{S} \backslash \mathcal{F}_{j}$ and $1 \leq i, j \leq n$ and $i \neq j$.

(vi) $h_{0} \cdot\left(v_{i}-v_{0}\right)>0$ for all $1 \leq i \leq n$.

(vii) The vectors $\left\{v_{1}-v_{0}, \ldots, v_{n}-v_{0}\right\}$ are a basis for $\mathbb{R}^{n}$.

(viii) The vectors $\left\{h_{1}, \ldots, h_{n}\right\}$ are a basis for $\mathbb{R}^{n}$.

(ix) There exist $\gamma_{1}>0, \ldots, \gamma_{n}>0$ such that $h_{0}=-\gamma_{1} h_{1}-\cdots-\gamma_{n} h_{n}$.

Proof. We present only the proofs for properties (viii)-(ix).

(viii) The implicit description of $\mathcal{S}$ says that there exist $\alpha_{0}, \ldots, \alpha_{n} \in \mathbb{R}$ such that $\mathcal{S}=$ $\left\{x \in \mathbb{R}^{n} \mid h_{i} \cdot x \leq \alpha_{i}, \quad \forall i \in\{0, \ldots, n\}\right\}$. Also, in this implicit description we have $\mathcal{F}_{i}=\left\{x \in \mathbb{R}^{n} \mid h_{i} \cdot x=\alpha_{i}\right\} \cap \mathcal{S}$. Assume there exist $c_{1}, \ldots, c_{n} \in \mathbb{R}$ such that $\sum_{j=1}^{n} c_{j} h_{j}=0$. It then follows that for all $i \in I, \sum_{j=1}^{n} c_{j} h_{j} \cdot\left(v_{i}-v_{0}\right)=0$. Since $v_{0}$ is contained in all facets except $\mathcal{F}_{0}$ and similarly $v_{i}$ is contained in all facets except $\mathcal{F}_{i}$, we have

$$
\sum_{j=1}^{n} c_{j} h_{j} \cdot\left(v_{i}-v_{0}\right)=\sum_{j=1}^{n} c_{j}\left(h_{j} \cdot v_{i}-\alpha_{j}\right)=c_{i}\left(h_{i} \cdot v_{i}-\alpha_{i}\right)=0
$$

But $h_{i} \cdot v_{i}<\alpha_{i}$ and hence $h_{i} \cdot v_{i}-\alpha_{i}<0$. Therefore $c_{i}=0, \forall i \in I$. We conclude $\left\{h_{1}, \ldots, h_{n}\right\}$ are linearly independent.

(ix) We again use the implicit description of $\mathcal{S}$ in which $\mathcal{S}=\left\{x \in \mathbb{R}^{n} \mid h_{i} \cdot x \leq \alpha_{i}, \quad \forall i \in\right.$ $\{0, \ldots, n\}\}$ and $\mathcal{F}_{i}=\left\{x \in \mathbb{R}^{n} \mid h_{i} \cdot x=\alpha_{i}\right\} \cap \mathcal{S}$. By (vii), $\mathbb{R}^{n}=\operatorname{span}\left\{h_{1}, \ldots, h_{n}\right\}$, so there exist $\gamma_{1}, \ldots, \gamma_{n} \in \mathbb{R}$ such that

$$
h_{0}=\sum_{j=1}^{n} \gamma_{j} h_{j}
$$


Applying (v) we have for each $i \in I$

$$
h_{0} \cdot\left(v_{i}-v_{0}\right)=\sum_{j=1}^{n} \gamma_{j} h_{j} \cdot\left(v_{i}-v_{0}\right)=\sum_{j=1}^{n} \gamma_{j}\left(h_{j} \cdot v_{i}-\alpha_{j}\right)=\gamma_{i}\left(h_{i} \cdot v_{i}-\alpha_{i}\right)>0
$$

But $h_{i} \cdot v_{i}<\alpha_{i}$, so $\gamma_{i}<0$ for each $i \in I$.

\subsection{Placing Triangulation}

In this section we describe a method to triangulate a polytopic space with respect to another space. Let $\mathcal{P}$ be a polytope and let $\mathcal{O}$ be an affine space of dimension less than $n$. We assume $\mathcal{P} \cap \mathcal{O}$ is a polytope with vertices $V_{\mathcal{O}}:=\left\{o_{1}, \ldots, o_{r}\right\}$. We want to triangulate $\mathcal{P}$ with respect to $\mathcal{O}$.

First, we define an ordered point set $V:=\left\{v_{1}, \ldots, v_{p}\right\}$ such that $\mathcal{P}=$ co $(V)$ and the first $r$ points of $V$ are $V_{\mathcal{O}}$. Note that not every element of $V_{\mathcal{O}}$ need be a vertex of $\mathcal{P}$. Now we propose a triangulation of $\mathcal{P}$ which will have the feature that $\mathcal{O}$ can only lie in lower dimensional faces of simplices of the triangulation. We use a standard procedure called the placing triangulation (see $[18,17]$ ). To describe this triangulation method we need a few definitions.

Suppose $V$ is a finite set of points such that $\mathcal{P}=\operatorname{co}(V)$ is an $n$-dimensional polytope. A subdivision of $V$ is a finite collection $\mathbb{S}=\left\{\mathcal{P}_{1}, \ldots, \mathcal{P}_{q}\right\}$ of $n$-dimensional polytopes such that: (1) The vertices of each $\mathcal{P}_{i}$ are drawn from $V$ (though not every point in $V$ need be used); (2) $\mathcal{P}=\cup_{i} \mathcal{P}_{i}$; (3) If $i \neq j$, then $\mathcal{P}_{i} \cap \mathcal{P}_{j}$ is a common (possibly empty) face of the boundaries of $\mathcal{P}_{i}$ and $\mathcal{P}_{j}$.

Definition 2.1. Let $x \in \mathbb{R}^{n}, \mathcal{P}$ an $n$-dimensional polytope, and $\mathcal{F}$ a facet of $\mathcal{P}$. The hyperplane $\mathcal{H}=\operatorname{aff}(\mathcal{F})$ defines an open half-space containing $\operatorname{int}(\mathcal{P})$. If $x$ is contained in the opposite open half-space, then $\mathcal{F}$ is said to be visible from $x$. (If $\mathcal{P}$ is a $k$-dimensional 
polytope in $\mathbb{R}^{n}$ with $k<n$ and $x \in$ aff $(\mathcal{P})$, then the ambient space is viewed to be aff $(\mathcal{P})$.)

Now we can describe what it means to place a vertex. Let $\mathbb{S}=\left\{\mathcal{P}_{1}, \ldots, \mathcal{P}_{q}\right\}$ be a subdivision of $V$ and $v \in \mathbb{R}^{n}$ such that $v \notin V$.

Definition 2.2. The subdivision $\mathbb{T}$ of $V \cup\{v\}$ that results from placing $v$ is obtained as follows:

1. If $v \notin$ aff $(V)$, then for each $\mathcal{P}_{i} \in \mathbb{S}$, include co $\left(\mathcal{P}_{i} \cup\{v\}\right)$ in $\mathbb{T}$.

2. If $v \in$ aff $(V)$, then for each $\mathcal{P}_{i} \in \mathbb{S}, \mathcal{P}_{i} \in \mathbb{T}$ and if $\mathcal{F}$ is a facet of $\mathcal{P}_{i}$ that is contained in a facet of co $(V)$ visible from $v$, then co $(\mathcal{F} \cup\{v\}) \in \mathbb{T}$.

Theorem 2.1. [18] Suppose $V$ is a finite set of points such that $V_{\mathcal{O}} \subset V$ and $\mathcal{P}=\operatorname{co}(V)$ is an $n$-dimensional polytope. If the points of $V$ are ordered such that $\left\{o_{1}, \ldots, o_{r}\right\}$, the vertices of $\mathcal{P} \cap \mathcal{O}$, are listed first and if $\mathbb{T}$ is the subdivision obtained by placing the points of $V$ in order, then $\mathbb{T}$ is a triangulation of $V$ such that for every $n$-dimensional simplex $\mathcal{S} \in \mathbb{T}$, $\operatorname{int}(\mathcal{S}) \cap \mathcal{O}=\emptyset$ and if $\mathcal{S} \cap \mathcal{O} \neq \emptyset$, then $\mathcal{S} \cap \mathcal{O}$ is a face of $\mathcal{S}$.

\section{$2.4 \mathscr{M}$-Matrices}

We say a matrix $M$ is a $\mathscr{Z}$-matrix if the off-diagonal elements are non-positive; i.e. $m_{i j} \leq 0$ for all $i \neq j[4]$. A special case of a $\mathscr{Z}$-matrix is a nonsingular $\mathscr{M}$. A nonsingular $\mathscr{M}$-matrix is a $\mathscr{Z}$-matrix with eigenvalues whose real parts are positive. The following theorem characterizes non-singular $\mathscr{M}$-matrices. For a exhaustive treatment see [4], Chapter 6 .

Theorem 2.2. Let $M \in \mathbb{R}^{k \times k}$ be a $\mathscr{Z}$-matrix. Then the following are equivalent:

(i) $M$ is a non-singular $\mathscr{M}$-matrix.

(ii) $\Re(\lambda)>0$ for all eigenvalues $\lambda$ of $M$. 
(iii) There exists a vector $\xi \succeq 0$ in $\mathbb{R}^{k}$ such that $M \xi \succ 0$.

(iv) The inequalities $y \succeq 0$ and $M y \preceq 0$ have only the trivial solution $y=0$, and $M$ is non-singular.

(v) $M$ is monotone; that is, $M y \succeq 0$ implies $y \succeq 0$ for all $y \in \mathbb{R}^{k}$.

(vi) $M$ is nonsingular and $M^{-1}$ is a non-negative matrix.

\subsection{Convex Analysis}

The following background is extracted from [21]. Let $\mathcal{C}$ be a non-empty convex set in $\mathbb{R}^{n}$. We say that $\mathcal{C}$ recedes in direction $v \in \mathbb{R}^{n}$ if

$$
(\exists x \in \mathcal{C})\{x+\lambda v \mid \lambda \geq 0\} \subset \mathcal{C}
$$

Equivalently, $v$ is said to be a direction of recession of $\mathcal{C}$.

Let $\mathcal{C}_{1}$ and $\mathcal{C}_{2}$ be non-empty sets in $\mathbb{R}^{n}$. A hyperplane $\mathcal{H}$ is said to separate $\mathcal{C}_{1}$ and $\mathcal{C}_{2}$ if $\mathcal{C}_{1}$ is contained in one of the closed half-spaces associated with $\mathcal{H}$ and $\mathcal{C}_{2}$ lies in the opposite closed half-space. It is said to separate $\mathcal{C}_{1}$ and $\mathcal{C}_{2}$ properly if $\mathcal{C}_{1}$ and $\mathcal{C}_{2}$ are not both actually contained in $\mathcal{H}$ itself. It is said to separate $\mathcal{C}_{1}$ and $\mathcal{C}_{2}$ strongly if there exists some $\epsilon>0$ such that $\mathcal{C}_{1}+\epsilon \mathscr{B}$ is contained in one of the open half-spaces associated with $\mathcal{H}$, and $\mathcal{C}_{2}+\epsilon \mathscr{B}$ is contained in the opposite half-space.

Theorem 2.3. Let $\mathcal{C}_{1}$ and $\mathcal{C}_{2}$ be non-empty convex sets in $\mathbb{R}^{n}$. In order that there exist a hyperplane $\mathcal{H}$ separating $\mathcal{C}_{1}$ and $\mathcal{C}_{2}$ strongly, it is necessary and sufficient that

$$
\inf \left\{\left\|x_{1}-x_{2}\right\| \mid x_{1} \in \mathcal{C}_{1}, x_{2} \in \mathcal{C}_{2}\right\}>0
$$

The relative interior of a convex set $\mathcal{C}$ in $\mathbb{R}^{n}$, denoted by ri $\mathcal{C}$, is defined as the interior of $\mathcal{C}$ which results when $\mathcal{C}$ is regarded as a subset of its affine hull aff $\mathcal{C}$. A convex set $\mathcal{C}$ is said to be relatively open if ri $\mathcal{C}=\mathcal{C}$. 
Theorem 2.4. Let $\mathcal{C}_{1}$ be a non-empty relatively open convex set in $\mathbb{R}^{n}$, and let $\mathcal{C}_{2}$ be a non-empty affine set in $\mathbb{R}^{n}$ not meeting $\mathcal{C}_{1}$. Then there exists a hyperplane $\mathcal{H}$ containing $\mathcal{C}_{2}$ such that one of the open half-spaces associated with $\mathcal{H}$ contains $\mathcal{C}_{1}$.

Theorem 2.5. Let $\mathcal{C}_{1}$ and $\mathcal{C}_{2}$ be non-empty disjoint closed convex sets in $\mathbb{R}^{n}$ having no commmon directions of recession. Then there exists a hyperplane separating $\mathcal{C}_{1}$ and $\mathcal{C}_{2}$ strongly.

Theorem 2.6. Let $\mathcal{C}_{1}$ and $\mathcal{C}_{2}$ be non-empty convex sets in $\mathbb{R}^{n}$ whose closures are disjoint. If either set is bounded, there exists a hyperplane separating $\mathcal{C}_{1}$ and $\mathcal{C}_{2}$ strongly.

\subsection{Nonsmooth Analysis}

A set-valued map $\mathcal{Y}: \mathbb{R}^{n} \rightarrow 2^{\mathbb{R}^{q}}$ is said to be upper semicontinuous at $x \in \mathbb{R}^{n}$ if for all $\epsilon>0$ there exists $\delta>0$ such that

$$
\left\|x-x^{\prime}\right\|<\delta \quad \Longrightarrow \mathcal{Y}\left(x^{\prime}\right) \subset \mathcal{Y}(x)+\epsilon \mathscr{B}
$$

Lemma 2.2. Consider the set-valued map

$$
\mathcal{Y}(x):=\left\{A x+B u+a \mid u \in \mathbb{R}^{m}\right\}
$$

$\mathcal{Y}(x)$ is upper semicontinuous.

Proof. Let $L:=\|A\|$ and fix $\epsilon>0$. Select $\delta=\frac{\epsilon}{L}$. Let $x, x^{\prime} \in \mathbb{R}^{n}$ be such that $\left\|x-x^{\prime}\right\|<\delta$. Let $y^{\prime} \in \mathcal{Y}\left(x^{\prime}\right)$ be arbitrary. There exists $u^{\prime} \in \mathbb{R}^{m}$ such that $y^{\prime}=A x^{\prime}+B u^{\prime}+a$. Now consider $y:=A x+B u^{\prime}+a \in \mathcal{Y}(x)$. We have

$$
\begin{aligned}
\left\|y-y^{\prime}\right\| & =\left\|A x+B u^{\prime}+a-A x^{\prime}-B u^{\prime}-a\right\| \\
& \leq\|A\|\left\|x-x^{\prime}\right\| \\
& \leq L \cdot \frac{\epsilon}{L}=\epsilon .
\end{aligned}
$$


Since $y^{\prime} \in \mathcal{Y}\left(x^{\prime}\right)$ is arbitrary, we obtain

$$
\mathcal{Y}\left(x^{\prime}\right) \subset \mathcal{Y}(x)+\epsilon \mathscr{B}
$$

as desired.

Let $\mathcal{S} \subset \mathbb{R}^{n}$ be a closed set. Define the distance function

$$
d_{\mathcal{S}}(x):=\inf \{\|x-y\| \mid y \in \mathcal{S}\}
$$

The Bouligand tangent cone (or simply tangent cone) to $\mathcal{S}$ at $x$, denoted $T_{\mathcal{S}}(x)$, is defined as

$$
T_{\mathcal{S}}(x):=\left\{v \in \mathbb{R}^{n} \mid \liminf _{t \downarrow 0} \frac{d_{\mathcal{S}}(x+t v)}{t}=0\right\}
$$

The following is a useful characterization of the tangent cone (which is sometimes taken as the definition).

Lemma 2.3. Let $\mathcal{S}$ be a closed set in $\mathbb{R}^{n}$. Let $\left\{x^{k}\right\}$ be a sequence such that $x^{k} \in \mathcal{S}$ and $\lim _{k \rightarrow \infty} x^{k}=x$. Also, let $\left\{t^{k}\right\}$ be a sequence such that $t^{k}>0$ and $\lim _{k \rightarrow \infty} t^{k}=0$. Then

$$
\lim _{k \rightarrow \infty} \frac{x^{k}-x}{t^{k}} \in T_{\mathcal{S}}(x)
$$

Theorem 2.7. Consider the differential equation

$$
\dot{x}=f(x)
$$

where $f: \mathbb{R}^{n} \rightarrow \mathbb{R}^{n}$ is Lipschitz continuous. Let $\mathcal{P}$ be a compact, convex set. If for all $x_{0} \in \mathcal{P}$, solutions of (2.2) satisfy $x(t) \in \mathcal{P}, \forall t \geq 0$, then $\mathcal{P}$ contains an equilibrium of $(2.2)$.

\subsection{Fixed Point Theory}

\subsubsection{Sperner's Lemma}

Let $\mathbb{T}$ be a triangulation of $n$-dimensional simplex $\mathcal{S}$. A proper labeling of the vertices of $\mathbb{T}$ is as follows: 
(P1) Vertices of the original simplex $\mathcal{S}$ have $n+1$ distinct labels.

(P2) Vertices of $\mathbb{T}$ on a face of $\mathcal{S}$ are labeled using only the labels of the vertices forming the face.

Given a properly labeled triangulation of $\mathcal{S}$, we say a simplex in $\mathbb{T}$ is distinguished if its vertices have all $n+1$ labels.

Lemma 2.4 (Sperner). Every properly labeled triangulation of $\mathcal{S}$ has an odd number of distinguished simplices.

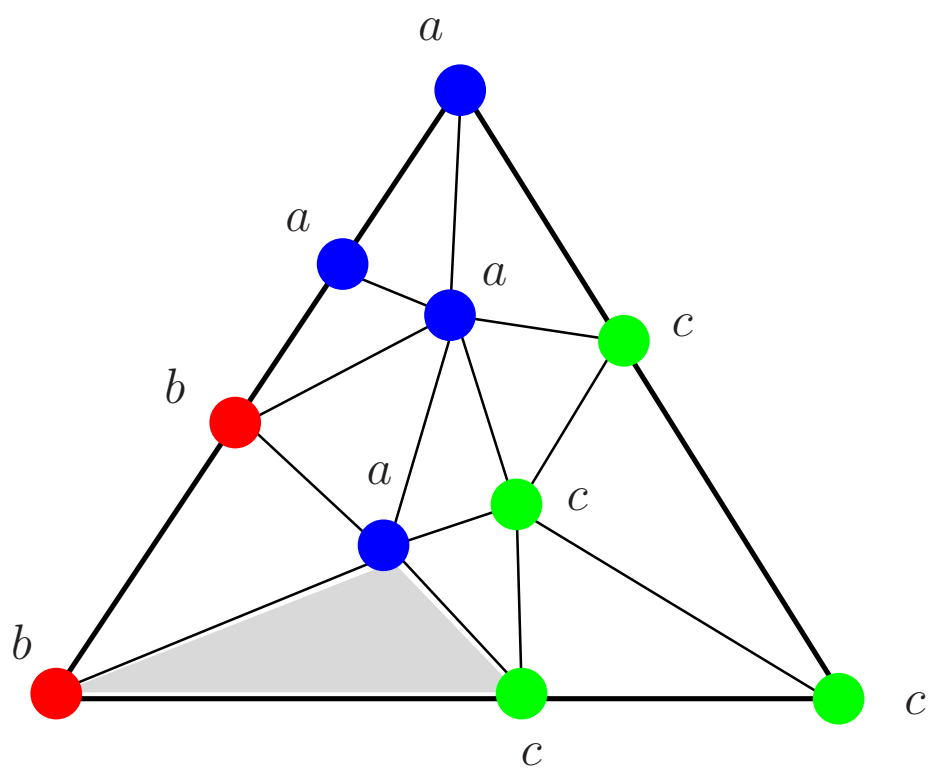

Figure 2.1: Example of Sperner's Lemma

Example 2.1. By way of example, consider the simplex $\mathcal{S}$ in Figure 2.1 and suppose the possible labels are $a$ (blue), $b$ (red), or $c$ (green). The vertices each have a distinct label, so condition (P1) is met. Also, for the shown triangulation of $\mathcal{S},(\mathrm{P} 2)$ is satisfied. For example, along the left edge, vertices are labelled only by $a$ or $b$. Consequently there exists at least one distinguished subsimplex, shaded in the figure, with vertices with all three labels. 


\section{Chapter 3}

\section{Reach Control: Basic Principles}

This thesis studies the reach control problem on simplices. The problem is for an affine system defined on a simplex to reach a prespecified facet of the simplex in finite time. In this chapter we explore basic principles which shape the features of the problem. These principles are derived from the geometry of the simplex and from convexity properties of affine systems.

\subsection{Reach Control Problem}

We study an $n$-dimensional simplex defined by

$$
\mathcal{S}:=\operatorname{co}\left\{v_{0}, \ldots, v_{n}\right\}
$$

where $v_{i} \in \mathbb{R}^{n}$ are its vertices. Define the vertex set

$$
V:=\left\{v_{0}, \ldots, v_{n}\right\} .
$$

We denote the $(n-1)$-dimensional facets by $\mathcal{F}_{0}, \ldots, \mathcal{F}_{n}$, where the index of each facet is determined by the vertex it does not contain. Consider the affine control system defined on $\mathcal{S}$ :

$$
\dot{x}=A x+a+B u, \quad x \in \mathcal{S},
$$


where $A \in \mathbb{R}^{n \times n}, a \in \mathbb{R}^{n}, B \in \mathbb{R}^{n \times m}$, and $\operatorname{rank}(B)=m$. Let $\phi_{u}\left(t, x_{0}\right)$ be the trajectory of (3.1) under a control $u(t)$ starting from $x_{0} \in \mathcal{S}$ and evaluated at time $t$. We are interested in studying reachability of the target $\mathcal{F}_{0}$ from $\mathcal{S}$. By a control assignment $\mathscr{U}$ we mean either an assignment of an open-loop control $u(t)$ for each initial condition $x_{0} \in \mathcal{S}$, or a feedback control $u(x)$ which is defined on all of $\mathcal{S}$. Let $u_{x}$ denote the value of control used at a point $x \in \mathcal{S}$ associated with control assignment $\mathscr{U}$.

Problem 3.1 (Reach Control Problem (RCP)). Consider system (3.1) defined on $\mathcal{S}$. Find a control assignment $\mathscr{U}$ such that:

(i) For every $x_{0} \in \mathcal{S}$ there exist $T \geq 0$ and $\gamma>0$ such that $\phi_{u}\left(t, x_{0}\right) \in \mathcal{S}$ for all $t \in[0, T], \phi_{u}\left(T, x_{0}\right) \in \mathcal{F}_{0}$, and $\phi_{u}\left(t, x_{0}\right) \notin \mathcal{S}$ for all $t \in(T, T+\gamma)$.

(ii) There exists $\varepsilon>0$ such that for all $x \in \mathcal{S}$, and for all $u_{x}$ corresponding to the control assigment $\mathscr{U}, \quad\left\|A x+a+B u_{x}\right\|>\varepsilon$.

Condition (i) of RCP ensures that trajectories of (3.1) starting from initial conditions in $\mathcal{S}$ reach the target $\mathcal{F}_{0}$ in finite time, while not first leaving $\mathcal{S}$. Condition (ii) is a robustness condition that ensures that trajectories leave $\mathcal{S}$ with sufficient speed.

Definition 3.1. A point $x_{0} \in \mathcal{S}$ can reach $\mathcal{F}_{0}$ with constraint in $\mathcal{S}$ with control type $\mathbb{U}$, denoted by $x_{0} \stackrel{\mathcal{S}}{\longrightarrow} \mathcal{F}_{0}$, if there exists a control $u$ of type $\mathbb{U}$ such that properties (i)-(ii) of Problem 3.1 hold. We write $\mathcal{S} \stackrel{\mathcal{S}}{\longrightarrow} \mathcal{F}_{0}$ by control type $\mathbb{U}$ if for every $x_{0} \in \mathcal{S}, x_{0} \stackrel{\mathcal{S}}{\longrightarrow} \mathcal{F}_{0}$ with control of type $\mathbb{U}$.

Let $\mathcal{B}=\operatorname{Im} B$, the image of $B$. Define the set

$$
\mathcal{O}:=\left\{x \in \mathbb{R}^{n}: A x+a \in \mathcal{B}\right\}
$$

Notice that the vector field $A x+a+B u$ can vanish at any $x \in \mathcal{O}$ for an appropriate choice of $u \in \mathbb{R}^{m}$, so $\mathcal{O}$ is the set of all possible equilibrium points of (3.1). Thus, if $x_{0}$ 
is an equilibrium of (3.1) under feedback control, then $x_{0} \in \mathcal{O}$. We also define the set of possible equilibrium points of (3.1) on $\mathcal{S}$ by

$$
\mathcal{G}:=\mathcal{S} \cap \mathcal{O}
$$

Associated with $\mathcal{G}$ is its vertex index set

$$
I_{\mathcal{G}}:=\left\{i: v_{i} \in V \cap \mathcal{G}\right\}
$$

The following lemma provides a characterization of $\mathcal{O}$.

\section{Lemma 3.1.}

(i) If $\operatorname{Im}(A) \subseteq \mathcal{B}$ and $a \notin \mathcal{B}$, then $\mathcal{O}=\emptyset$;

(ii) If $\operatorname{Im}(A) \subseteq \mathcal{B}$ and $a \in \mathcal{B}$, then $\mathcal{O}=\mathbb{R}^{n}$;

(iii) Otherwise, $\mathcal{O}$ is an affine space.

Lemma 3.2. Let $(A, B)$ be a controllable pair. If $\beta \in \operatorname{Ker}\left(B^{T}\right) \cap \operatorname{Ker}\left(A^{T}\right)$, then $\beta=0$.

Proof. By duality, if $(A, B)$ is controllable, then $\left(B^{T}, A^{T}\right)$ is observable. Thus, $\mathcal{N}:=$ $\cap_{i=1}^{n} \operatorname{Ker}\left(B^{T}\left(A^{T}\right)^{i-1}\right)=0$. Suppose there is $\beta \neq 0$ such that $B^{T} \beta=0$ and $A^{T} \beta=0$. Then $B^{T}\left(A^{T}\right)^{k} \beta=0$, for all $k \in \mathbb{N}^{+}$, so $0 \neq \beta \in \mathcal{N}$, a contradiction.

Lemma 3.3. If $(A, B)$ is controllable, then $\mathcal{O}$ is an affine subspace with $\operatorname{dim}(\mathcal{O})=m$.

Proof. Let $\mathcal{B}=\operatorname{span}\left\{b_{1}, \ldots, b_{m}\right\}$ and choose a basis

$$
\mathbb{R}^{n}=\operatorname{span}\left\{b_{1}, \ldots, b_{m}, \beta_{1}, \ldots, \beta_{n-m}\right\}
$$

with $\beta_{j} \cdot b_{i}=0$. Then

$$
\mathcal{O}=\left\{x \in \mathbb{R}^{n} \mid \beta_{i} \cdot(A x+a)=0, i=1, \ldots, n-m\right\} .
$$

Thus, $\mathcal{O}$ is determined by $n-m$ affine constraints. The result is obtained if we can show the constraints are independent. First, we must show the row vectors $\left\{\beta_{1}^{T} A, \ldots, \beta_{n-m}^{T} A\right\}$ 
are linearly independent. Suppose not. Then there exist constants $c_{1}, \ldots, c_{n-m}$, not all zero, such that

$$
c_{1} \beta_{1}^{T} A+\cdots+c_{n-m} \beta_{n-m}^{T} A=0 .
$$

Let $\beta:=c_{1} \beta_{1}+\cdots+c_{n-m} \beta_{n-m} \neq 0$. By (3.4), $\beta \in \operatorname{Ker}\left(A^{T}\right)$ and $\beta \in \operatorname{Ker}\left(B^{T}\right)$. This contradicts Lemma 3.2.

Finally, we must show

$$
\left(\beta_{1} \cdot a, \ldots, \beta_{n-m} \cdot a\right) \in \operatorname{Im}\left[\begin{array}{c}
-\beta_{1}^{T}- \\
\vdots \\
-\beta_{n-m}^{T}-
\end{array}\right] A .
$$

Since $(A, B)$ is controllable, $a \in \operatorname{Im}(A)+\mathcal{B}$. Thus, $a=a_{1}+a_{2}$, with $a_{1} \in \mathcal{B}$ and $a_{2} \in \operatorname{Im}(A)$. Then $\beta_{i} \cdot a=\beta_{i} \cdot a_{2}$ so we get

$$
\left(\left[\begin{array}{c}
-\beta_{1}^{T}- \\
\vdots \\
-\beta_{n-m}^{T}-
\end{array}\right] a_{2}\right) \in \operatorname{Im}\left(\left[\begin{array}{c}
-\beta_{1}^{T}- \\
\vdots \\
-\beta_{n-m}^{T}-
\end{array}\right] A\right)
$$

as desired.

\subsection{Necessary Conditions}

In this section we investigate necessary conditions for solvability of RCP using open-loop controls. We say that a function $u:[0, \infty) \rightarrow \mathbb{R}^{m}$ is an open-loop control if it is bounded on any compact interval and it is measurable. By Caratheodory's theorem, solutions of (3.1) using open-loop controls exist and are unique.

Let $h_{i} \in \mathbb{R}^{n}$ be the unit normal vector to each facet $\mathcal{F}_{i}$ pointing outside of $\mathcal{S}$. Define the index sets

$$
I:=\{1, \ldots, n\}
$$

and $I_{i}:=I \backslash\{i\}$, where $I_{0}=I$. Define the closed, convex cone $\mathcal{C}_{i}$ at $v_{i} \in V$ by

$$
\mathcal{C}_{i}:=\left\{y \in \mathbb{R}^{n}: h_{j} \cdot y \leq 0, j \in I_{i}\right\} .
$$




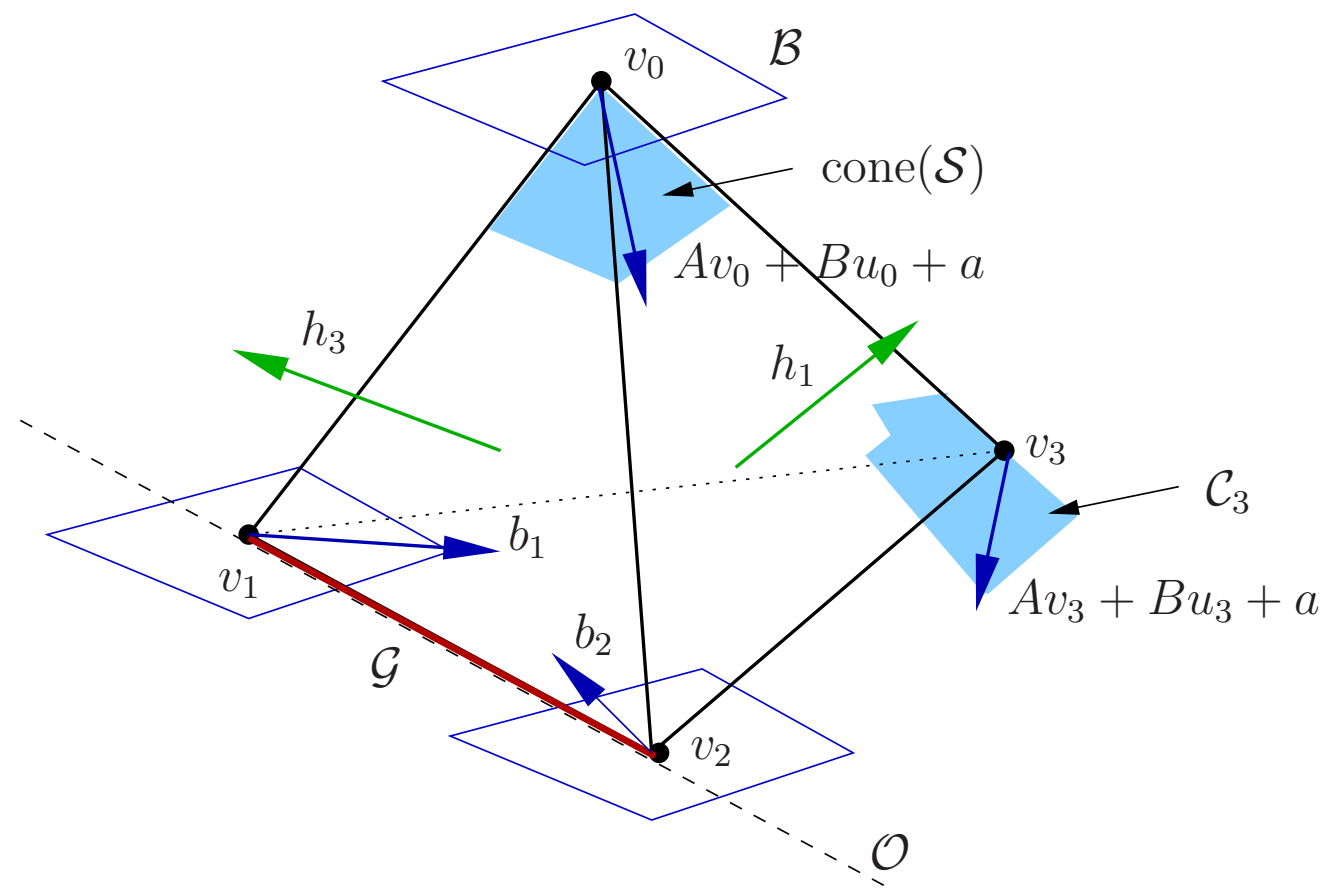

Figure 3.1: Geometric constructs for the reach control problem.

Also define

$$
\operatorname{cone}(\mathcal{S}):=\mathcal{C}_{0}=\operatorname{cone}\left\{v_{1}-v_{0}, \ldots, v_{n}-v_{0}\right\}
$$

Note that cone $(\mathcal{S})$ is the tangent cone to $\mathcal{S}$ at $v_{0}$.

Definition 3.2. We say the invariance conditions are solvable if there exist $u_{0}, \ldots, u_{n} \in$ $\mathbb{R}^{m}$ such that

$$
A v_{i}+a+B u_{i} \in \mathcal{C}_{i} .
$$

Equivalently,

$$
h_{j} \cdot\left(A v_{i}+a+B u_{i}\right) \leq 0, \quad i \in\{0, \ldots, n\}, \quad j \in I_{i} .
$$

We will see in the sequel that the invariance conditions (3.5) are required for constructing affine feedbacks. For general state feedbacks, stronger conditions are needed.

Definition 3.3. We say a state feedback $u(x)$ satisfies the invariance conditions if for 
all $j \in I$ and $x \in \mathcal{F}_{j}$,

$$
h_{j} \cdot(A x+B u(x)+a) \leq 0 .
$$

Example 3.1. Figure 3.1 illustrates the definitions so far for the case $n=3$ and $m=2$. We have a simplex $\mathcal{S}$ with normal vectors $h_{i}$ to each facet $\mathcal{F}_{i}$. Depicted by a shaded section is cone $(\mathcal{S})$, the tangent cone at $v_{0}$. The space $\mathcal{B}$ is copied to $v_{0}$, and in this view we see that $\mathcal{B} \cap \operatorname{cone}(\mathcal{S})=\mathbf{0}$. That is, $\mathcal{B}$ does not "dip" into the tangent cone at $v_{0}$. The set $\mathcal{O}$ intersects $\mathcal{S}$ along the face $\overline{v_{1} v_{2}}$, and this forms $\mathcal{G}$. It is interpreted as the set of possible equilibria of the system. We know that in $\mathcal{G}$, the only velocity vectors available to the closed loop system are vectors in $\mathcal{B}$. This is depicted by placing copies of $\mathcal{B}$ at each of the vertices of $\mathcal{G}$. Two velocity vectors $b_{1}$ and $b_{2}$ are shown, and these clearly satisfy the invariance conditions at $v_{1}$ and $v_{2}$, respectively. At vertices not in $\mathcal{G}$, the drift term $A x+a$ becomes relevant, and the figure depicts closed-loop velocity vectors at $v_{0}, v_{3} \notin \mathcal{G}$ which satisfy their respective invariance conditions. The invariance conditions can be interpreted in terms of the cones $\mathcal{C}_{i}$. Consider vertex $v_{3}$ where $\mathcal{C}_{3}$ is depicted by a shaded region. This cone is shaped like an open book whose spine is parallel to the face $\overline{v_{0} v_{3}}$ and whose cover and back cover lie in $\mathcal{F}_{2}$ and $\mathcal{F}_{1}$, respectively. The invariance condition at $v_{3}$ is satisfied if the closed-loop velocity vector $A v_{3}+B u_{3}+a$ lies in $\mathcal{C}_{3}$.

The invariance conditions are central to the solution of RCP on simplices, as seen by the next result.

Theorem 3.1. Suppose $\mathcal{S} \stackrel{\mathcal{S}}{\longrightarrow} \mathcal{F}_{0}$ by open-loop controls. Then the invariance conditions (3.5) are solvable.

Proof. Define $\mathcal{Y}(x):=\left\{A x+B u+a \mid u \in \mathbb{R}^{m}\right\}$. Let $x_{0} \in \mathcal{S} \backslash \mathcal{F}_{0}$. By assumption there exists $u(t)$ and a time $T>0$ such that $\phi_{u}\left(t, x_{0}\right) \in \mathcal{S}$ for all $t \in[0, T]$. Since $u(\cdot)$ is an open-loop control, there exists $c>0$ such that $\|u(t)\| \leq c$, for all $t \in[0, T]$. Consider the set $\mathcal{Y}_{c}(x):=\left\{A x+B u+a \mid u \in \mathbb{R}^{m},\|u\| \leq c\right\}$. It is easy to show that both $x \mapsto \mathcal{Y}_{c}(x)$ and $x \mapsto \mathcal{Y}(x)$ are upper semicontinuous. Now take a sequence $\left\{t_{i} \mid t_{i} \in(0, T]\right\}$ with $t_{i} \downarrow 0$. 
Since $\left\{v \in \mathcal{Y}_{c}(x) \mid x \in \mathbb{R}^{n}\right\}$ is uniformly bounded on $\mathcal{S}$, there exists $M>0$ such that $\left\|\phi_{u}\left(t_{i}, x_{0}\right)-x_{0}\right\| \leq M t_{i}$. Therefore $\left\{\frac{\phi_{u}\left(t_{i}, x_{0}\right)-x_{0}}{t_{i}}\right\}$ is a bounded sequence and there exists a convergence subsequence (with indices relabeled) such that $\lim _{i \rightarrow \infty} \frac{\phi_{u}\left(t_{i}, x_{0}\right)-x_{0}}{t_{i}}=v$. Since $\phi_{u}\left(t_{i}, x_{0}\right) \in \mathcal{S}$, by the definition of the Bouligand tangent cone, $v \in T_{\mathcal{S}}\left(x_{0}\right)$. Now we show $v \in \mathcal{Y}\left(x_{0}\right)$.

We have

$$
\frac{\phi_{u}\left(t_{i}, x_{0}\right)-x_{0}}{t_{i}}=\frac{1}{t_{i}} \int_{0}^{t_{i}}\left[A \phi_{u}\left(\tau, x_{0}\right)+B u(\tau)+a\right] d \tau .
$$

Let

$$
\begin{aligned}
y_{0} & :=A x_{0}+B u(0)+a \in \mathcal{Y}\left(x_{0}\right) \\
y(\tau) & :=A \phi_{u}\left(\tau, x_{0}\right)+B u(\tau)+a \in \mathcal{Y}\left(\phi_{u}\left(\tau, x_{0}\right)\right)
\end{aligned}
$$

By the upper semicontinuity of $\mathcal{Y}(x)$, given $\epsilon>0$, there exists $\delta>0$ such that if $\left\|x_{0}-\phi_{u}\left(\tau, x_{0}\right)\right\|<\delta$, then $\left\|y_{0}-y(\tau)\right\|<\epsilon$. Equivalently for $i$ sufficiently large and for all $\tau \in\left[0, t_{i}\right],\left\|y_{0}-y(\tau)\right\|<\epsilon$. This can be rewritten as : for $i$ sufficiently large and for all $\tau \in\left[0, t_{i}\right]$,

$$
y(\tau)=y_{0}+\rho(\tau)
$$

for some function $\rho(\tau)$ satisfying $\left\|y_{0}-y(\tau)\right\|<\epsilon$, for all $\tau \in\left[0, t_{i}\right]$. Thus, for $i$ sufficiently large

$$
\frac{1}{t_{i}} \int_{0}^{t_{i}} y(\tau) d \tau=\frac{1}{t_{i}} \int_{0}^{t_{i}}\left(y_{0}+\rho(\tau)\right) d \tau=y_{0}+\frac{1}{t_{i}} \int_{0}^{t_{i}} \rho(\tau) d \tau .
$$

However,

$$
\begin{aligned}
\left\|\frac{1}{t_{i}} \int_{0}^{t_{i}} \rho(\tau) d \tau\right\| & \leq \frac{1}{t_{i}} \int_{0}^{t_{i}}\|\rho(\tau)\| d \tau \\
& \leq \frac{1}{t_{i}} \cdot \epsilon t_{i}=\epsilon
\end{aligned}
$$

We conclude, for $i$ sufficiently large

$$
\frac{1}{t_{i}} \int_{0}^{t_{i}}\left[A \phi_{u}\left(\tau, x_{0}\right)+B u(\tau)+a\right] d \tau \in \mathcal{Y}\left(x_{0}\right)+\epsilon \mathscr{B}
$$


Using (3.7), this gives for $i$ sufficiently large

$$
\frac{\phi_{u}\left(t_{i}, x_{0}\right)-x_{0}}{t_{i}} \in \mathcal{Y}\left(x_{0}\right)+\epsilon \mathscr{B} .
$$

Since $\mathcal{Y}\left(x_{0}\right)$ is a closed subset of $\mathbb{R}^{n}, v \in \mathcal{Y}\left(x_{0}\right)+\epsilon \overline{\mathscr{B}}$, and since $\epsilon$ is arbitrary, $v \in \mathcal{Y}\left(x_{0}\right)$. We conclude that

$$
\mathcal{Y}\left(x_{0}\right) \cap T_{\mathcal{S}}\left(x_{0}\right) \neq \emptyset, \quad x_{0} \in \mathcal{S} \backslash \mathcal{F}_{0} .
$$

Since $T_{\mathcal{S}}\left(v_{0}\right)=\operatorname{cone}(\mathcal{S})$, and $T_{\mathcal{S}}(x)=\mathcal{C}_{i}$ for $x \in\left(v_{0}, v_{i}\right)$, it follows that the invariance conditions are solvable at $v_{0}$ and along edges $\left(v_{0}, v_{i}\right), i \in I$.

Now consider $v_{i}, i \in I$. If $v_{i} \in \mathcal{O}$, then the invariance conditions are solvable by selecting $u_{i} \in \mathbb{R}^{m}$ such that $A v_{i}+B u_{i}+a=0$. Instead suppose $v_{i} \notin \mathcal{O}$. Suppose by way of contradiction that $\mathcal{Y}\left(v_{i}\right) \cap \mathcal{C}_{i}=\emptyset$. Both $\mathcal{Y}\left(v_{i}\right)$ and $\mathcal{C}_{i}$ are non-empty closed convex sets in $\mathbb{R}^{n}$ and by assumption they have no common directions of recession. By Theorem 2.5, they are strongly separated. That is, there exists $\epsilon>0$ such that

$$
\inf _{y \in \mathcal{Y}\left(v_{i}\right), w \in \mathcal{C}_{i}}\|y-w\|>\epsilon .
$$

By the upper semicontinuity of $\mathcal{Y}(\cdot)$, there exists $\delta>0$ such that if $\left\|x-v_{i}\right\|<\delta$, then $\mathcal{Y}(x) \subset \mathcal{Y}\left(v_{i}\right)+\frac{\epsilon}{2} \mathscr{B}$. In particular, taking $x \in\left(v_{0}, v_{i}\right)$, we get $\mathcal{Y}(x) \cap \mathcal{C}_{i}=\emptyset$, a contradiction.

The next result says that if a controller abides by the invariance conditions at a vertex of $\mathcal{S}$, then an equilibrium of the controlled system may not be automatically induced at that vertex.

Theorem 3.2. If $\mathcal{S} \stackrel{\mathcal{S}}{\longrightarrow} \mathcal{F}_{0}$ by open-loop controls, then for all vertices $v_{i} \in \mathcal{O}$,

$$
\mathcal{B} \cap \mathcal{C}_{i} \neq \mathbf{0}
$$

Proof. Consider $v_{i} \in \mathcal{O}$. Suppose by way of contradiction that $\mathcal{B} \cap \mathcal{C}_{i}=\mathbf{0}$. Since $A v_{i}+a \in \mathcal{B}$, there exists $u_{i} \in \mathbb{R}^{m}$ such that $A v_{i}+a+B u_{i}=0$. By condition (ii) of RCP and by Theorem 3.1, there exists $\varepsilon>0$ such that for all $x \in \mathcal{S}$, there exists $u_{x} \in \mathbb{R}^{m}$ such that 
- $A x+B u_{x}+a \in T_{\mathcal{S}}(x)$ if $x \in \mathcal{S} \backslash \mathcal{F}_{0}$;

- $\left\|A x+B u_{x}+a\right\|>\varepsilon$.

By continuity there exists $\delta>0$ such that if $\left\|x-v_{i}\right\|<\delta$, then $\left\|A x+a+B u_{i}\right\|<\varepsilon / 2$. Thus, it must be that $\left\|B\left(u_{x}-u_{i}\right)\right\|>\varepsilon / 2$ for all $x \in \mathcal{S}$ satisfying $\left\|x-v_{i}\right\|<\delta$. Since $\mathcal{C}_{i}$ is a closed cone, there exists $\alpha>0$ such that if $b \in \mathcal{B}$ satisfies $\|b\|>\varepsilon / 2$, then $b+\alpha \mathscr{B} \notin \mathcal{C}_{i}$. In particular, we can choose $x \in\left(v_{0}, v_{i}\right)$ sufficiently close to $v_{i}$ such that $\left\|A x+a+B u_{i}\right\|<\min \{\alpha, \epsilon / 2\}$. Then

$$
A x+B u_{x}+a=\left(A x+a+B u_{i}\right)+B\left(u_{x}-u_{i}\right) \notin \mathcal{C}_{i},
$$

a contradiction.

\subsection{Affine Systems}

In this section we explore properties of affine systems on compact, convex sets. First we examine the relationship between positively invariant sets, existence of equilibria, and trajectories leaving the set in finite time. Second we explore the way in which trajectories exit a compact, convex set when invariance conditions such as (3.5) hold.

Let $\mathcal{P} \subset \mathbb{R}^{n}$ be a compact, convex set and consider the affine system defined on $\mathcal{P}$ :

$$
\dot{x}=A x+a, \quad x \in \mathcal{P},
$$

where $A \in \mathbb{R}^{n \times n}$ and $a \in \mathbb{R}^{n}$. Let $\phi\left(t, x_{0}\right)$ denote the unique trajectory of (3.9) starting from $x_{0} \in \mathcal{P}$.

Lemma 3.4. Consider the affine system (3.9) defined on a compact, convex set $\mathcal{P}$. Suppose there exists $x_{0} \in \mathcal{P}$ such that $\phi\left(t, x_{0}\right) \in \mathcal{P}$ for all $t \geq 0$. Then the set

$$
\Phi:=\operatorname{co}\left\{\phi\left(t, x_{0}\right) \mid t \geq 0\right\} \subset \mathcal{P}
$$

is a positively invariant set. Moreover, $\Phi$ contains an equilibrium of (3.9), i.e. there exists $\bar{x} \in \Phi$ such that $A \bar{x}+a=0$. 
Proof. Consider any $x_{1} \in \Phi$. Then there exist $w_{0}, \ldots, w_{n} \in\left\{\phi\left(t, x_{0}\right) \mid t \geq 0\right\}$ and $\alpha_{i} \in[0,1]$ with $\sum_{i} \alpha_{i}=1$ such that $x_{1}=\sum_{i=0}^{n} \alpha_{i} w_{i}$. Since (3.9) is affine, we have

$$
\phi\left(t, x_{1}\right)=\sum_{i=0}^{n} \alpha_{i} \phi\left(t, w_{i}\right), \quad t \geq 0 .
$$

Also, there exist $t_{i} \geq 0$ such that $w_{i}=\phi\left(t_{i}, x_{0}\right)$. Thus

$$
\phi\left(t, x_{1}\right)=\sum_{i=0}^{n} \alpha_{i} \phi\left(t, \phi\left(t_{i}, x_{0}\right)\right)=\sum_{i=0}^{n} \alpha_{i} \phi\left(t+t_{i}, x_{0}\right) \in \Phi, \quad t \geq 0 .
$$

By continuity with respect to initial conditions for (3.9), we have that $\bar{\Phi}$, the closure of $\Phi$, is also positively invariant. Then by Theorem $2.7, \Phi \subset \mathcal{P}$ contains an equilibrium of (3.9).

Lemma 3.5. Consider the affine system (3.9) defined on a compact, convex set $\mathcal{P}$. We have $A x+a \neq 0$ for all $x \in \mathcal{P}$ if and only if there exists $\xi \in \mathbb{R}^{n}$ such that

$$
\xi \cdot(A x+a)<0, \quad x \in \mathcal{P} .
$$

Proof. $\quad(\Longleftarrow)$ If $\xi \cdot(A x+a)<0$ for all $x \in \mathcal{P}$, then clearly $A x+a \neq 0$ for all $x \in \mathcal{P}$.

$(\Longrightarrow)$ Since $\mathcal{P}$ is compact and convex it follows that the image of $\mathcal{P}$ under the map $x \mapsto A x+a$, denoted by $\mathcal{C}_{1}=A \mathcal{P}+a$ is also compact and convex, and by assumption, it does not contain the origin. Let $\mathcal{C}_{2}:=\mathbf{0}$. Then we can apply Theorem 2.6 to obtain that there exists a hyperplane $\mathcal{H}$ that separates $\mathcal{C}_{1}$ and $\mathcal{C}_{2}$ strongly. In other words, there exists $\epsilon>0$ and some $\xi \in \mathbb{R}^{n}$ such that for all $x \in \mathcal{P}, \xi \cdot(A x+a) \leq-\epsilon$, or $\xi \cdot(A x+a)<0$.

We call the equation (3.10) a flow condition for system (3.9) on $\mathcal{P}$. A consequence of the existence of a flow condition on a compact, convex set is that all trajectories of (3.9) originating in the set eventually leave it.

Lemma 3.6. Consider the affine system (3.9) defined on a compact, convex set $\mathcal{P}$. Suppose that for all $x \in \mathcal{P}, A x+a \neq 0$. Then for each $x_{0} \in \mathcal{P}$, the trajectory starting at $x_{0}$ eventually leaves $\mathcal{P}$, i.e. there exists $t_{1}>0$ such that $\phi\left(t_{1}, x_{0}\right) \notin \mathcal{P}$. 
Proof. Suppose $A x+a \neq 0$, for all $x \in \mathcal{P}$. From Lemma 3.5 there exists a vector $\xi$ and a constant $\epsilon>0$ such that for all $x \in \mathcal{P}, \xi \cdot(A x+a) \leq-\epsilon$. Suppose there exists $x_{0} \in \mathcal{P}$ such that $\phi\left(t, x_{0}\right)$ lies in $\mathcal{P}$ for all $t \geq 0$. Then

$$
\xi \cdot\left(A \phi\left(t, x_{0}\right)+a\right) \leq-\epsilon, \quad \forall t \geq 0 .
$$

Integrating both sides, we obtain

$$
\xi \cdot \phi\left(t, x_{0}\right) \leq \xi \cdot x_{0}-\epsilon t, \quad \forall t \geq 0 .
$$

This contradicts the compactness of $\mathcal{P}$.

The existence of a flow condition is also related to the set $\mathcal{O}$ for an affine control system. To see this, consider again the control system (3.1). The next result shows that a flow condition naturally arises on any compact, convex set that does not intersect $\mathcal{O}$. Importantly, the control input plays no role and, moreover, the origin of the vector $\xi$ can be made explicit.

Lemma 3.7. Consider the affine control system (3.1) defined on a compact, convex set $\mathcal{P}$. If $\mathcal{P} \cap \mathcal{O}=\emptyset$, then there exists $\xi \in \operatorname{Ker} B^{T}$ such that

$$
\xi \cdot(A x+a)<0, \quad \forall x \in \mathcal{P} .
$$

Proof. As in Lemma 3.5, we know that the image of $\mathcal{P}$ under the map $x \mapsto A x+a$ is compact and convex, and we denote it $\mathcal{C}_{1}=A \mathcal{P}+a$. First we show that $\mathcal{B} \cap \mathcal{C}_{1}=\emptyset$. Suppose not. That is, there is a point $x \in \mathcal{P}$ such that $A x+a \in \mathcal{B}$. Then $x \in \mathcal{O}$, by definition, which contradicts $\mathcal{O} \cap \mathcal{P}=\emptyset$. Since both $\mathcal{B}$ and $\mathcal{C}_{1}$ are convex sets, by Theorem 2.6 there exists a hyperplane $\mathcal{H}$ separating $\mathcal{B}$ and $\mathcal{C}_{1}$ strongly. This implies $\mathcal{B}$ is parallel to $\mathcal{H}$ since $\mathcal{B}$ is a subspace. Let $\xi$ be the normal vector to $\mathcal{H}$ pointing to the side containing $\mathcal{B}$. Then, $\xi \in \operatorname{Ker} B^{T}$ and $\xi \cdot(A x+a)<0$ for all $x \in \mathcal{P}$.

Lemma 3.8. Consider the affine control system (3.1) defined on a compact, convex set $\mathcal{P}$. If ri $(\mathcal{P}) \cap \mathcal{O}=\emptyset$, then there exists $\xi \in \operatorname{Ker} B^{T}$ such that

$$
\xi \cdot(A x+a)<0, \quad \forall x \in \operatorname{ri} \mathcal{P} .
$$


Proof. By the same argument as in Lemma 3.7, $\mathcal{B} \cap \mathcal{C}_{1}=\emptyset$, where $\mathcal{C}_{1}=\operatorname{Ari}(\mathcal{P})+a$. Since both $\mathcal{B}$ and $\mathcal{C}_{1}$ are convex sets, and since $\mathcal{C}_{1}$ is relative open, by Theorem 2.4 there exists a hyperplane $\mathcal{H}$ separating $\mathcal{B}$ and $\mathcal{C}_{1}$ properly. This implies $\mathcal{B}$ is parallel to $\mathcal{H}$ since $\mathcal{B}$ is a subspace. Let $\xi$ be the normal vector to $\mathcal{H}$ pointing to the side containing $\mathcal{B}$. Then, $\xi \in \operatorname{Ker} B^{T}$ and $\xi \cdot(A x+a)<0$ for all $x \in \operatorname{ri} \mathcal{P}$.

Finally, we examine the way that trajectories of an affine system exit a compact, convex set when invariance conditions such as (3.5) or (3.6) hold. The following result is quite standard and the proof is omitted.

Lemma 3.9. Consider the affine system (3.9) defined on a compact, convex set $\mathcal{P}$. Suppose additionally that $\mathcal{P}$ is a polytope with facets $\left\{\mathcal{F}_{0}, \ldots, \mathcal{F}_{k}\right\}$. Let $h_{i}$ be the outward normal vector of $\mathcal{F}_{i}$. Suppose that for some facet $\mathcal{F}_{i}$ the following conditions hold:

$$
h_{i} \cdot(A x+a) \leq 0, \quad \forall x \in \mathcal{F}_{i}
$$

Then all trajectories originating in $\mathcal{P}$ that leave $\mathcal{P}$ do so via a facet $\mathcal{F}_{j}, j \neq i$.

The next result shows that for affine systems, if trajectories exit from the proper target facet, than the invariance conditions hold. The argument is a minor adaptation of Theorem 3.1, where it is shown that the invariance conditions must be solvable (but need not actually hold).

Lemma 3.10. Consider the affine system (3.9) defined on a compact, convex set $\mathcal{P}$. Suppose additionally that $\mathcal{P}$ is a polytope with facets $\left\{\mathcal{F}_{0}, \ldots, \mathcal{F}_{k}\right\}$. Let $h_{i}$ be the outward normal vector of $\mathcal{F}_{i}$. Suppose that $\mathcal{P} \stackrel{\mathcal{P}}{\longrightarrow} \mathcal{F}_{0}$. Then

$$
h_{i} \cdot(A x+a) \leq 0, \quad \forall x \in \mathcal{F}_{i}, i=1, \ldots, k
$$

Proof. Let $x_{0} \in \mathcal{P} \backslash \mathcal{F}_{0}$. By assumption there exists $T>0$ such that $\phi_{u}\left(t, x_{0}\right) \in \mathcal{P}$ for all $t \in[0, T]$. Take a sequence $\left\{t_{i} \mid t_{i} \in(0, T]\right\}$ with $t_{i} \downarrow 0$. Since $\left\{A x+a \mid x \in \mathbb{R}^{n}\right\}$ is uniformly bounded on $\mathcal{P}$, there exists $M>0$ such that $\left\|\phi\left(t_{i}, x_{0}\right)-x_{0}\right\| \leq M t_{i}$. Therefore 
$\left\{\frac{\phi\left(t_{i}, x_{0}\right)-x_{0}}{t_{i}}\right\}$ is a bounded sequence and there exists a convergence subsequence (with indices relabeled) such that $\lim _{i \rightarrow \infty} \frac{\phi\left(t_{i}, x_{0}\right)-x_{0}}{t_{i}}=v$. Since $\phi\left(t_{i}, x_{0}\right) \in \mathcal{P}$, by the definition of the Bouligand tangent cone, $v \in T_{\mathcal{P}}\left(x_{0}\right)$. However, we also know that

$$
\lim _{t_{i} \rightarrow 0} \frac{\phi\left(t_{i}, x_{0}\right)-x_{0}}{t_{i}}=:\left.\frac{d \phi\left(t, x_{0}\right)}{d t}\right|_{t=0}=A x_{0}+a .
$$

Thus, $A x_{0}+a \in T_{\mathcal{P}}\left(x_{0}\right)$, for all $x_{0} \in \mathcal{P} \backslash \mathcal{F}_{0}$. From the definition of the tangent cone, this implies

$$
h_{i} \cdot\left(A x_{0}+a\right) \leq 0, \quad x_{0} \in \mathcal{F}_{i} \backslash \mathcal{F}_{0}, \quad i=1, \ldots, k
$$

These inequalities extend to points in $\mathcal{F}_{0}$ by continuity of the functions $x \mapsto h_{i} \cdot(A x+$ a).

\subsection{Affine Feedback}

In this section we use the properties of affine systems to propose a solution of RCP by affine feedback. It relies on a synthesis procedure described in the next result.

Lemma 3.11. Consider two sets of points $V=\left\{v_{0}, \ldots, v_{n} \mid v_{i} \in \mathbb{R}^{n}\right\}$ and $\left\{u_{0}, \ldots, u_{n} \mid u_{i} \in\right.$ $\left.\mathbb{R}^{m}\right\}$. Suppose $V$ is affinely independent. Then there exist unique matrices $K \in \mathbb{R}^{m \times n}$ and $g \in \mathbb{R}^{m}$ such that

$$
u_{i}=K v_{i}+g, \quad i \in\{0, \ldots, n\} .
$$

Proof. We must show there exist matrices $K$ and $g$ such that

$$
\left[\begin{array}{ll}
v_{0}^{T} & 1 \\
\vdots & \\
v_{n}^{T} & 1
\end{array}\right]\left[\begin{array}{l}
K^{T} \\
g^{T}
\end{array}\right]=\left[\begin{array}{l}
u_{0}^{T} \\
\vdots \\
u_{n}^{T}
\end{array}\right] .
$$

If the $(n+1) \times(n+1)$ left-hand matrix is full rank, then multiplying by its inverse yields 
the unique solutions $K$ and $g$. However,

$$
\operatorname{rank}\left[\begin{array}{ll}
v_{0}^{T} & 1 \\
\vdots & \\
v_{n}^{T} & 1
\end{array}\right]=1+\operatorname{rank}\left[\begin{array}{l}
v_{1}^{T}-v_{0}^{T} \\
v_{2}^{T}-v_{0}^{T} \\
\vdots \\
v_{n}^{T}-v_{0}^{T}
\end{array}\right]=1+n .
$$

The last equality follows since the points $\left\{v_{0}, \ldots, v_{n}\right\}$ are affinely independent if and only if $\left\{v_{1}-v_{0}, \ldots, v_{n}-v_{0}\right\}$ are linearly independent.

Using the results in Section 3.3 and the synthesis procedure of Lemma 3.11, we obtain necessary and sufficient conditions for solvability of RCP by affine feedback.

Theorem 3.3. Given the system (3.1) and an affine feedback $u(x)=K x+g$, where $K \in \mathbb{R}^{m \times n}, g \in \mathbb{R}^{m}$, and $u_{0}=u\left(v_{0}\right), \ldots, u_{n}=u\left(v_{n}\right)$, the closed-loop system satisfies $\mathcal{S} \stackrel{\mathcal{S}}{\longrightarrow} \mathcal{F}_{0}$ if and only if

(a) The invariance conditions (3.5) hold.

(b) There is no equilibrium in $\mathcal{S}$.

Proof.

$\left(\Rightarrow\right.$ If $\mathcal{S} \stackrel{\mathcal{S}}{\longrightarrow} \mathcal{F}_{0}$ by affine feedback, then clearly the closed-loop system does not have equilibria in $\mathcal{S}$, for otherwise trajectories starting at an equilibrium would not leave $\mathcal{S}$. The invariance conditions (3.5) hold by Lemma 3.10.

$(\Leftarrow)$ By assumption, for vertex set $V$ there exist inputs $\left\{u_{0}, \ldots, u_{n}\right\}$ satisfying the invariance conditions (3.5). Invoking Lemma 3.11, there exists an affine feedback $u=$ $K x+g$ such that the invariance conditions are satisfied at the vertices. The resulting closed-loop system is

$$
\dot{x}=(A+B K) x+(B g+a)=\tilde{A} x+\tilde{a} .
$$


By assumption, $\tilde{A} x+\tilde{a} \neq 0$ for all $x \in \mathcal{S}$, so by Lemma 3.6, all trajectories leave $\mathcal{S}$ in finite time. From (3.5),

$$
h_{j} \cdot\left(\tilde{A} v_{i}+\tilde{a}\right) \leq 0, \quad i \in\{0, \ldots, n\}, \quad j \in I_{i} .
$$

By convexity

$$
h_{i} \cdot(\tilde{A} x+\tilde{a}) \leq 0, \quad \forall x \in \mathcal{F}_{i}, \quad i \in I
$$

By Lemma 3.9 trajectories cannot leave $\mathcal{S}$ via $\mathcal{F}_{1}, \ldots, \mathcal{F}_{n}$. This proves condition (i) of RCP. For condition (ii), since $\|\tilde{A} x+\tilde{a}\| \neq 0$ for all $x \in \mathcal{S}, \mathcal{S}$ is compact, and $x \mapsto\|\tilde{A} x+\tilde{a}\|$ is continuous, there exists $\varepsilon>0$ such that $\|\tilde{A} x+\tilde{a}\|>\varepsilon$ for all $x \in \mathcal{S}$.

Theorem 3.3 gives conditions for the solvability of RCP which are primarily of theoretical interest. In practice, these conditions do not realize a synthesis procedure, for one cannot guarantee that a proposed affine feedback satisfying the invariance conditions does not place closed-loop equilibria in $\mathcal{S}$. On the other hand, existence of a flow condition guarantees there are no equilibria in $\mathcal{S}$ by Lemma 3.5, so one can replace the requirement of no closed-loop equilibria with existence of a flow condition. This observation leads to an alternative set of necessary and sufficient conditions for solvability of RCP.

Theorem 3.4. $\mathcal{S} \stackrel{\mathcal{S}}{\longrightarrow} \mathcal{F}_{0}$ by affine feedback if and only if there exist $u_{0}, \ldots, u_{n} \in \mathbb{R}^{m}$ and $\xi \in \mathbb{R}^{n}$ such that

(a) The invariance conditions (3.5) hold.

(b) The flow condition holds: $\quad \xi \cdot\left(A v_{i}+B u_{i}+a\right)<0, \quad i \in\{0, \ldots, n\}$.

Theorem 3.4 can be viewed as a computational solution to the problem. If the invariance and flow conditions can be solved simultaneously for the unknowns $\xi \in \mathbb{R}^{n}$ and $u_{i} \in \mathbb{R}^{m}$, then an affine feedback can be constructed by the procedure of Lemma 3.11. Unfortunately, this approach relies on solving bilinear inequalities, which are known to be $N P$-hard [24]. 


\section{Chapter 4}

\section{Triangulations and Affine Feedback}

In this chapter we investigate RCP under the assumption of a special triangulation of the state space. This triangulation is selected in order to be able to obtain more geometric necessary and sufficient conditions for solvability of RCP. Some necessary conditions have already been presented in Section 3.2. This chapter instead focuses on geometric sufficient conditions for solvability by affine feedback. The motivation for doing so is clarified next.

\subsection{Motivation}

Our goal is to develop a general methodology involving triangulations of polytopic state spaces. The triangulation scheme is typically determined by some triangulation software, and one verifies that a set of necessary and sufficient conditions for reach control on the simplices of the triangulation are satisfied. This information is used in a dynamic programming algorithm that constructs a reachability graph on the triangulation. This approach has two significant drawbacks. First, the necessary and sufficient conditions for reach control on each simplex (say using Theorem 3.3) are precisely the equations which the control must satisfy to solve the problem. In other words, the computation of the controller is inextricably linked to the solvability of RCP. Thus, while one may be able to 
solve for the controller, there is no structural information about the system which tells you why. Second, if the design fails, there is no indication whether a better triangulation exists which would solve the problem.

This situation can best be understood by comparison with pole placement for MIMO linear systems. One can say that pole placement is achievable if a certain formula for the closed-loop characteristic polynomial matches a desired characteristic polynomial, and from this algebraic problem one computes the desired gain matrix. Or one can say that pole placement is possible because a Kalman rank condition holds. The latter condition not only provides the structural information about the system that is needed to determine if the problem is solvable, but it is also a gateway to solving many other problems. It decouples the computation of the controller from the properties of the system which make that solution exist. Indeed, the dichotomy between solvability and computation is the central premise of geometric control theory [26]. Quoting from the preface of [26]:

"Instead of looking directly for a feedback law (say $u=F x)$ which would solve your synthesis problem if a solution exists, first characterize solvability as a verifiable property of some constructible state subspace, say $Y$. Then, if all is well, you may calculate F from Y quite easily."

The results of Chapter 3 have provided the second step: you may calculate $u=K x+g$ quite easily to solve RCP. The first step, to characterize solvability as a verifiable property of the system together with the simplex has not been addressed, and it is the focus of this chapter.

\subsection{Affine Feedback}

Consider again a simplex $\mathcal{S}=$ co $\left\{v_{0}, v_{1}, \ldots, v_{n}\right\}$ and an affine system defined on $\mathcal{S}$

$$
\dot{x}=A x+B u+a, \quad x \in \mathcal{S},
$$


where $A \in \mathbb{R}^{n \times n}, a \in \mathbb{R}^{n}, B \in \mathbb{R}^{n \times m}$, and $\operatorname{rank}(B)=m$.

We begin by exploring geometric sufficient conditions for existence of affine feedbacks solving RCP in terms of the sets $\mathcal{O}$ and $\mathcal{G}$, defined in (3.2) and (3.3), respectively. We have seen in Theorem 3.3 that the invariance conditions by themselves are generally not enough to establish that RCP is solvable by affine feedback. However, there is one extreme case when the invariance conditions are also sufficient to solve the problem. These depend on combining Theorem 3.3 with the fact that $\mathcal{O}$ is the only place in the state space where equilibria can appear.

Theorem 4.1. Suppose $\mathcal{G}=\emptyset$. If the invariance conditions are solvable, then $\mathcal{S} \stackrel{\mathcal{S}}{\longrightarrow} \mathcal{F}_{0}$ by affine feedback.

Proof. Select the control $u_{i} \in \mathbb{R}^{m}$ for each vertex $v_{i} \in V$ to satisfy the invariance conditions. Using the method of Section 3.4 and since the vertices are affinely independent one can find unique $K$ and $g$ corresponding to the affine feedback $u(x)=K x+g$ such that $u\left(v_{i}\right)=u_{i}, 0 \leq i \leq n$. We obtain the affine closed-loop system $\dot{x}=(A+B K) x+(a+B g)$. Since $\mathcal{O} \cap \mathcal{S}=\emptyset$, we know the closed-loop system has no equilibria in $\mathcal{S}$. Therefore, applying Theorem 3.3, the result is obtained.

In general it is difficult to extend results such as Theorem 4.1. Instead, we consider a special triangulation of the state space, first discussed in Section 2.3, which significantly simplifies the problem of finding geometric necessary and sufficient conditions for solvability of RCP. It is based on the following assumption.

Assumption 4.1. Simplex $\mathcal{S}$ and system (3.1) satisfy the following condition: if $\mathcal{G} \neq \emptyset$, then $\mathcal{G}$ is a $\kappa$-dimensional face of $\mathcal{S}$, where $0 \leq \kappa \leq n$.

Remark 4.1.1. We have discussed that there are three possibilities for $\mathcal{O}$. If $\mathcal{O}=\emptyset$, then one applies Theorem 4.1. If $\mathcal{O}$ is the entire state space then we will see later in Chapter 5 that there are easily derived necessary and sufficient conditions for solvability. The only 
interesting case is when $\mathcal{O}$ is a $\kappa$-dimensional affine subspace with $\kappa<n$. This case arises, for example, when $(A, B)$ is controllable, and then the placing triangulation can be applied.

We can now find several new sufficient conditions for existence of an affine feedback to solve RCP. The main idea is to exploit the fact that if the state space is triangulated with respect to $\mathcal{O}$, then using Lemma 3.7, a flow condition fulfilling Theorem 3.4 is (almost) achievable on $\mathcal{S}$.

Theorem 4.2. Suppose Assumption 4.1 holds and $\mathcal{G} \neq \emptyset$. Suppose the following conditions hold.

1. The invariance conditions (3.5) are solvable.

2. $\mathcal{B} \cap \operatorname{cone}(\mathcal{S}) \neq \mathbf{0}$.

Then $\mathcal{S} \stackrel{\mathcal{S}}{\longrightarrow} \mathcal{F}_{0}$ by affine feedback.

Proof. Let $\mathcal{G}=$ co $\left\{v_{i_{1}}, \ldots, v_{i_{\kappa+1}}\right\}$, a $\kappa$-dimensional facet of $\mathcal{S}$ where $0 \leq \kappa \leq n$. Thus, $I_{\mathcal{G}}=\left\{i_{1}, \ldots, i_{\kappa+1}\right\}$. Let $b \in \mathcal{B} \cap \operatorname{cone}(\mathcal{S}), b \neq 0$, and select control values $u_{i}$ such that $y\left(v_{i}\right)=A v_{i}+B u_{i}+a=b$ for all $i \in I_{\mathcal{G}}$ (notice this is always achievable for $v_{i} \in \mathcal{O}$ ). Clearly, by the assumption that $b \in \operatorname{cone}(\mathcal{S}), y\left(v_{i}\right)$ satisfies the invariance conditions for $v_{i} \in V \cap \mathcal{O}$. We can select the remaining controls $u_{i}$ for $i \in\{0, \ldots, n\} \backslash I_{\mathcal{G}}$ such that $y\left(v_{i}\right) \neq 0$ (since $v_{i} \notin \mathcal{O}$ ) and $y\left(v_{i}\right)$ satisfies the invariance conditions. Finally, using $\left\{u_{0}, \ldots, u_{n}\right\}$ and the synthesis procedure in Section 3.4, construct the affine feedback $u(x)=K x+g$.

Define the closed-loop vector field $y(x):=(A+B K) x+B g+a$. By convexity of $y(x)$, $y(x)=b \neq 0$ for $x \in \mathcal{G}$. Also, $y(x) \neq 0$ for all $x \in \mathcal{S} \backslash \mathcal{G}$ since equilibria only lie in $\mathcal{O}$. Applying Theorem 3.3, the result is obtained.

One can also obtain sufficient conditions for existence of affine feedback even when $\mathcal{B} \cap \operatorname{cone}(\mathcal{S})=\mathbf{0}$. Of course, this will only be possible if $v_{0} \notin \mathcal{G}$ by Theorem 3.2. This 
relies on the idea that there are enough degrees of freedom in $\mathcal{B}$ with respect to $\mathcal{G}$. We make the following assumptions.

\section{Assumption 4.2.}

$(\mathrm{A} 1) \mathcal{G}=\operatorname{co}\left\{v_{1}, \ldots, v_{\kappa+1}\right\}$, with $0 \leq \kappa<m$.

$(\mathrm{A} 2) \mathcal{B} \cap \operatorname{cone}(\mathcal{S})=\mathbf{0}$.

(A3) There exists a linearly independent set $\left\{b_{i} \in \mathcal{B} \cap \mathcal{C}_{i} \mid i \in I_{\mathcal{G}}\right\}$.

The important new assumption is (A3) which says that $\mathcal{B}$ and $\mathcal{G}$ are arranged with respect to each other so that there are enough degrees of freedom in $\mathcal{B}$ both to span a $\kappa+1$-dimensional subspace of $\mathcal{B}$ and at the same time satisfy all the invariance conditions for the vertices of $\mathcal{G}$. For this to work, it is of course necessary that $\kappa<m$. It is helpful to obtain some intuition as to why linear independence is a central property which determines whether or not an affine feedback exists in the case when $\mathcal{B} \cap \operatorname{cone}(\mathcal{S})=\mathbf{0}$. Also this intuition will help motivate the developments of Chapter 5 .

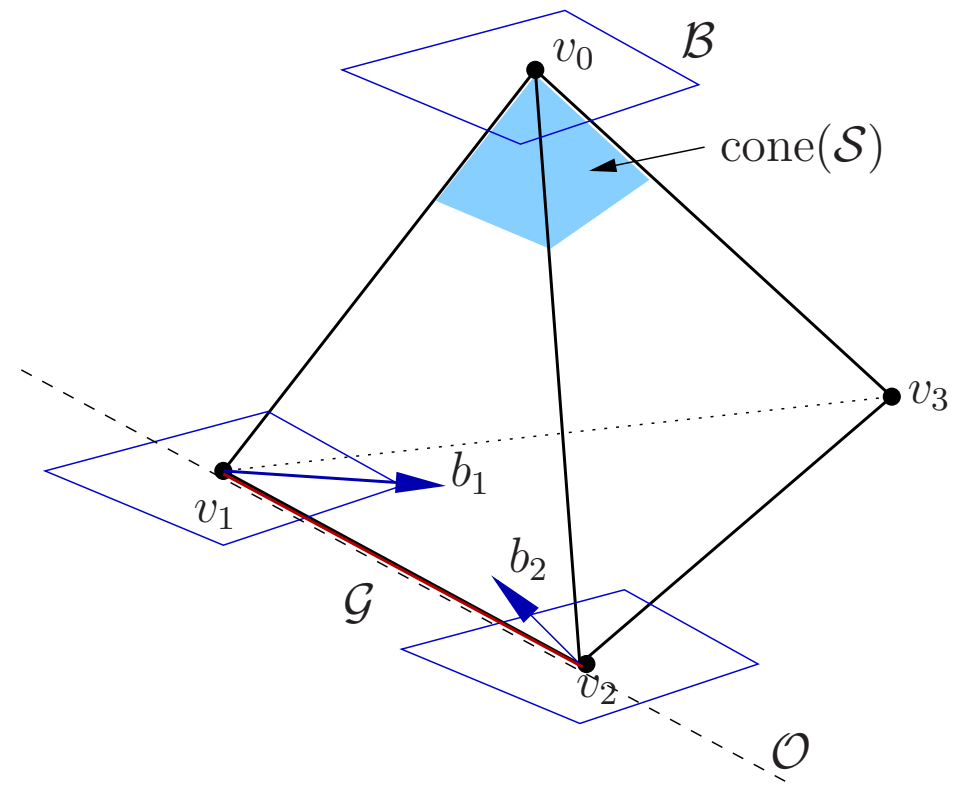

Figure 4.1: Continuous Assignment Along $\mathcal{G}$ of Vectors in $\mathcal{B}$. 
Consider Figure 4.1 in which $\mathcal{B} \cap \operatorname{cone}(\mathcal{S})=\mathbf{0}$ and $\mathcal{G}=\overline{v_{1} v_{2}}$. Suppose we find a linearly independent set $\left\{b_{1}, b_{2} \mid b_{i} \in \mathcal{B} \cap \mathcal{C}_{i}\right\}$ as shown in the figure (a copy of $\mathcal{B}$ is attached at each vertex). Then along $\left[v_{1}, v_{2}\right]$ we can always choose a feedback $u(x)$ such that the closed-loop vector field

$$
y(x):=A x+B u(x)+a=c_{1}(x) b_{1}+c_{2}(x) b_{2}, \quad c_{i}(x) \geq 0
$$

continuously interpolates between $y\left(v_{1}\right)=b_{1}$ and $y\left(v_{2}\right)=b_{2}$. This is also evident from examining the figure. Indeed with this controller, the invariance conditions are guaranteed to hold not only at $v_{1}$ and $v_{2}$ (by the definition of $\mathcal{C}_{i}$ ) but also on the open interval $\left(v_{1}, v_{2}\right)$. Namely, because $c_{i}(x) \geq 0$,

$$
h_{j} \cdot y(x) \leq 0, \quad j=3, \ldots, n
$$

Now consider the opposite situation as depicted in Figure 4.2. Again we have $\mathcal{B} \cap$ cone $(\mathcal{S})=\mathbf{0}$ and $\mathcal{G}=\overline{v_{1} v_{2}}$, but in this case $m=1$. Thus, for every choice of $b_{i} \in \mathcal{B} \cap \mathcal{C}_{i}$, $i=1,2$, we obtain that $\left\{b_{1}, b_{2}\right\}$ are linearly dependent. Pick any $b_{1} \in \mathcal{B} \cap \mathcal{C}_{1}$ with $b_{1} \neq 0$, as shown. Then we know that for any $b_{2} \in \mathcal{B} \cap \mathcal{C}_{2}$,

$$
b_{2}=c_{1} b_{1}, \quad c_{1} \in \mathbb{R} .
$$

Now check the invariance conditions at $v_{2}$. In particular, we have the invariance condition

$$
h_{1} \cdot b_{2}=c_{1}\left(h_{1} \cdot b_{1}\right) \leq 0
$$

If we have assumed that $\mathcal{B} \cap \operatorname{cone}(\mathcal{S})=\mathbf{0}$, then it must be that $h_{1} \cdot b_{1}>0$, for otherwise we would have $0 \neq b_{1} \in \mathcal{B} \cap \operatorname{cone}(S)$. Then from (4.3) we obtain that $c_{1} \leq 0$. This is illustrated in Figure 4.2, where $b_{2}$ points in the opposite direction of $b_{1}$. Consider a continuous vector field $y(x)$ on $\mathcal{S}$ and suppose we assign $y\left(v_{1}\right)=b_{1}$. Then we know that $y(x)$ is irrevocably constrained to be $y\left(v_{2}\right)=c_{1} b_{1}$ with $c_{1} \leq 0$. Now suppose that $y(x)$ continuously interpolates between $y\left(v_{1}\right)$ and $y\left(v_{2}\right)$ along $\left[v_{1}, v_{2}\right]$ using only $\left\{b_{1}, b_{2}\right\}$. Then along $\left[v_{1}, v_{2}\right], y(x)$ has the form:

$$
y(x)=c(x) b_{1},
$$




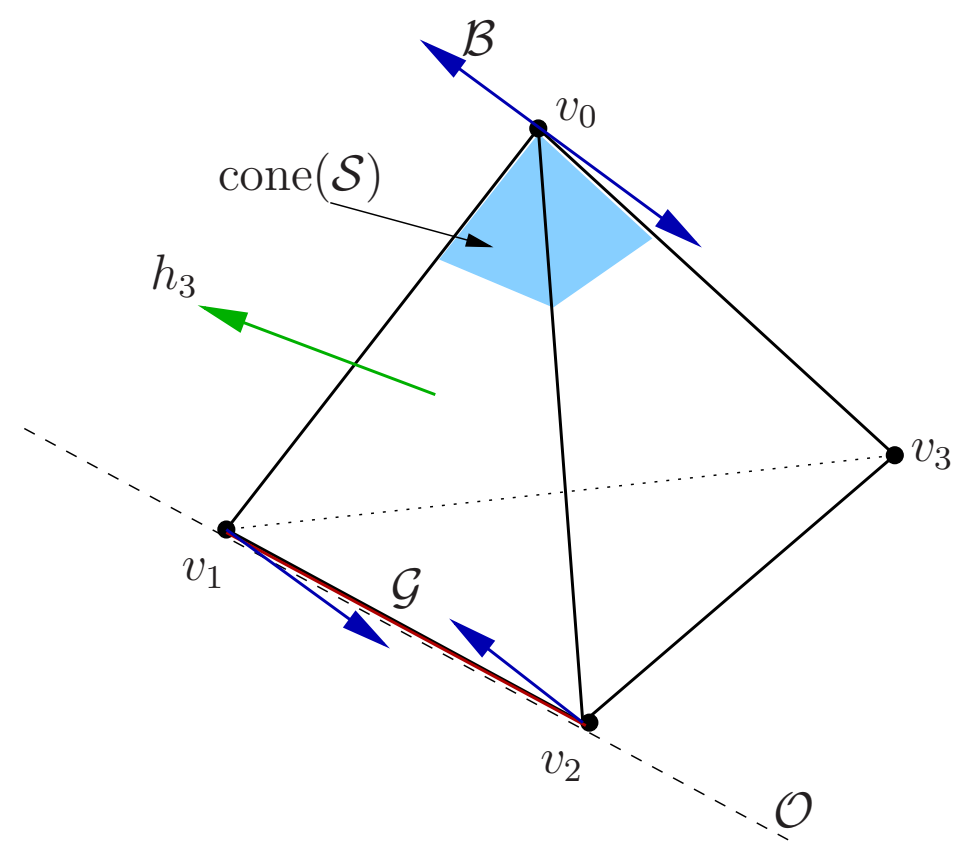

Figure 4.2: Not enough degrees of freedom in $\mathcal{B}$.

where $c(x)$ is a continuous function of $x \in\left[v_{1}, v_{2}\right]$ with $c\left(v_{1}\right)>0$ and $c\left(v_{2}\right) \leq 0$. By the Intermediate Value Theorem, there exists $\bar{x} \in\left[v_{1}, v_{2}\right]$ such that $c(\bar{x})=0$. Thus, there is an equilibrium along $\left[v_{1}, v_{2}\right]$ for the closed loop system.

Therefore, it is clear that $y(x)$ cannot simply interpolate between $\left\{b_{1}, b_{2}\right\}$ along $\left[v_{1}, v_{2}\right]$ and other directions in $\mathcal{B}$ must be invoked. This argument can now be carried on inductively to higher dimensions and in each dimension one finds that more degrees of freedom are needed in $\mathcal{B}$ to carrying out the continuous assignment of the vector field. Finally, the procedure either terminates with exhausting all the vertices of $\mathcal{G}$, without first exhausting the degrees of freedom in $\mathcal{B}$, or instead one first exhausts all the usable degrees of freedom in $\mathcal{B}$. This question of which is exhausted first determines a sharp boundary between existence of affine feedbacks and existence of equilibria.

Theorem 4.3. Suppose Assumption 4.1 holds and $\mathcal{G}=\operatorname{co}\left\{v_{1}, \ldots, v_{\kappa+1}\right\}$, with $0 \leq \kappa<$ m. Suppose the following conditions hold.

1. The invariance conditions (3.5) are solvable. 
2. There exists a linearly independent set $\left\{b_{i} \in \mathcal{B} \cap \mathcal{C}_{i} \mid i \in I_{\mathcal{G}}\right\}$.

Then $\mathcal{S} \stackrel{\mathcal{S}}{\longrightarrow} \mathcal{F}_{0}$ by affine feedback.

Proof. If $\mathcal{B} \cap \operatorname{cone}(\mathcal{S}) \neq \mathbf{0}$ then the result follows from Theorem 4.2. Therefore, we assume that $\mathcal{B} \cap \operatorname{cone}(\mathcal{S})=\mathbf{0}$. For all $i \in I_{\mathcal{G}}$, we assign control values $u_{i}$ such that $y\left(v_{i}\right)=A v_{i}+$ $B u_{i}+a=b_{i} \in \mathcal{B} \cap \mathcal{C}_{i}$. We can select the remaining controls $u_{i}$ for $i \in\{0, \ldots, n\} \backslash I_{\mathcal{G}}$ such that $y\left(v_{i}\right) \neq 0$ (since $v_{i} \notin \mathcal{O}$ ) and $y\left(v_{i}\right)$ satisfies the invariance conditions. Finally, using $\left\{u_{0}, \ldots, u_{n}\right\}$ and the synthesis procedure in Section 3.4, construct the affine feedback $u(x)=K x+g$.

We claim a flow condition holds for the closed loop vector field $y(x):=(A+B K) x+$ $B g+a$ on $\mathcal{G}$. To that end, let $\mathcal{C}_{1}:=\mathbf{0}$ and $\mathcal{C}_{2}:=\operatorname{co}\left\{b_{1}, \ldots, b_{\kappa+1}\right\}$. We observe that $\mathcal{C}_{1} \cap \mathcal{C}_{2}=\emptyset$, for if not, then there exist $\lambda_{i} \geq 0, \sum_{i} \lambda_{i}=1$ such that $0=\lambda_{1} b_{1}+\cdots+$ $\lambda_{\kappa+1} b_{\kappa+1}$. This contradicts that $\left\{b_{1}, \ldots, b_{\kappa+1}\right\}$ are linearly independent. Since $\mathcal{C}_{1}$ and $\mathcal{C}_{2}$ are compact, convex, and disjoint, we can apply Separating Hyperplane Theorem 2.6 to conclude that there exists a hyperplane $\mathcal{H}$ separating $\mathcal{C}_{1}$ and $\mathcal{C}_{2}$ strongly in $\mathcal{B}$. That is, there exists $\beta \in \mathcal{B}$ such that for all $y \in \mathcal{C}_{2}, \beta \cdot y<0$. By the convexity of $y(x)$, we have $\beta \cdot y(x)<0$ for all $x \in \mathcal{G}$. Clearly $y(x) \neq 0$ for $x \in \mathcal{G}$. Also, $y(x) \neq 0$ for all $x \in \mathcal{S} \backslash \mathcal{G}$ since equilibria only lie in $\mathcal{O}$. Applying Theorem 3.3, the result is obtained. 


\section{Chapter 5}

\section{Reach Control and Continuous State}

\section{Feedback}

The goal of this chapter is to explore solvability of RCP in cases where affine feedback cannot be used. Using a geometric approach, we identify the system structure that causes affine feedbacks to fail. This structure is encoded in so-called reach control indices. The first part of the chapter elucidates these indices. In the second part, we show that the failure to solve RCP is manifested in the appearance of equilibria inside the simplex. We show how the reach control indices can be used to isolate closed-loop equilibria. Finally, we conclude that if RCP is not solvable by affine feedback, then it is not solvable by continuous state feedback.

Based on the findings of the previous chapter, we focus on the following special case.

Assumption 5.1. Simplex $\mathcal{S}$ and system (3.1) satisfy the following conditions.

$(\mathrm{C} 1) \mathcal{G}=\operatorname{co}\left\{v_{1}, \ldots, v_{\kappa+1}\right\}$, with $0 \leq \kappa<n$.

$(\mathrm{C} 2) \mathcal{B} \cap \operatorname{cone}(\mathcal{S})=\mathbf{0}$.

(C3) The maximum number of linearly independent vectors in any set $\left\{b_{i} \in \mathcal{B} \cap \mathcal{C}_{i} \mid i \in\right.$ $\left.I_{\mathcal{G}}\right\}$ is $m^{\star}$ with $1 \leq m^{\star} \leq \kappa+1$. 
Assumption (C1) rules out the application of Theorem 4.1, and it enforces that $v_{0} \notin$ $\mathcal{O}$. The latter requirement is because, by Theorem 3.2, when $v_{0} \in \mathcal{O}$ and $(\mathrm{C} 2)$ holds, then RCP is not solvable. Assumption (C2) rules out the application of Theorem 4.2. Asssumption (C3) says there does not exist a full linearly independent set $\left\{b_{i} \in \mathcal{B} \cap \mathcal{C}_{i} \mid i \in\right.$ $I_{\mathcal{G}}$, in contrast with Assumption 4.2. Thus, Theorem 4.3 cannot be used. We remark that $m^{\star}$ is well-defined (for $\operatorname{dim}\left(\operatorname{span}\left\{b_{i} \in \mathcal{B} \cap \mathcal{C}_{i} \mid i \in I_{\mathcal{G}}\right\}\right) \in\{0, \ldots, \kappa+1\}$ defines a finite set of integers for which the maximum exists).

\subsection{Preliminaries}

We introduce a family of matrices which concisely characterize the invariance conditions on $\mathcal{G}$. Let $1 \leq p \leq q \leq \kappa+1$ and define

$$
M_{p, q}:=\left[\begin{array}{cccc}
\left(h_{p} \cdot b_{p}\right) & \left(h_{p} \cdot b_{p+1}\right) & \cdots & \left(h_{p} \cdot b_{q}\right) \\
\vdots & \vdots & & \vdots \\
\left(h_{q} \cdot b_{p}\right) & \left(h_{q} \cdot b_{p+1}\right) & \cdots & \left(h_{q} \cdot b_{q}\right)
\end{array}\right] \in \mathbb{R}^{(q-p+1) \times(q-p+1)}
$$

Define the matrices

$$
H_{p, q}:=\left[h_{p} \cdots h_{q}\right] \in \mathbb{R}^{n \times(q-p+1)}, \quad Y_{p, q}:=\left[b_{p} \cdots b_{q}\right] \in \mathbb{R}^{n \times(q-p+1)} .
$$

Then

$$
M_{p, q}=H_{p, q}^{T} Y_{p, q}
$$

Recall from Section 2.4 that $M$ is a $\mathscr{Z}$-matrix if the off-diagonal elements are nonpositive; i.e. $m_{i j} \leq 0$ for all $i \neq j$. Since $b_{i} \in \mathcal{B} \cap \mathcal{C}_{i}, i \in I_{\mathcal{G}}$, each $M_{p, q}$ is a $\mathscr{Z}$-matrix. Also under the condition that $\mathcal{B} \cap \operatorname{cone}(\mathcal{S})=\mathbf{0}, M_{p, q}$ adopts further algebraic properties.

Lemma 5.1. Suppose $\mathcal{B} \cap \operatorname{cone}(\mathcal{S})=0$. Let $1 \leq p \leq q \leq \kappa+1$ and suppose $\left\{b_{p}, \ldots, b_{q} \mid b_{i} \in\right.$ $\left.\mathcal{B} \cap \mathcal{C}_{i}\right\}$ are linearly independent. Then $M_{p, q}$ is a non-singular $\mathscr{M}$-matrix.

Proof. First we note that since $\operatorname{rank}\left(H_{p, q}\right)=q-p+1$ and by $\operatorname{assumption} \operatorname{rank}\left(Y_{p, q}\right)=$ $q-p+1$, we have that $M_{p, q}$ is non-singular. Next, we claim that $M_{p, q}$ has a positive 
diagonal; that is, $\left(M_{p, q}\right)_{i i}>0$ for $i=1, \ldots, q-p+1$. For if not, we would have $h_{j} \cdot b_{p+i-1} \leq 0$ for all $j=1, \ldots, n$, which implies $0 \neq b_{p+i-1} \in \mathcal{B} \cap \operatorname{cone}(\mathcal{S})$, a contradiction. Now suppose there exists $c \in \mathbb{R}^{q-p+1}$ with $c \neq 0$ and $c \succeq 0$ such that $M_{p, q} c \preceq 0$. Define the vector $\bar{y}=Y_{p, q} c \in \mathcal{B}$. Note that $\bar{y} \neq 0$ because $\left\{b_{p}, \ldots, b_{q}\right\}$ are linearly independent. Then $M_{p, q} c=H_{p, q}^{T} Y_{p, q} c=H_{p, q}^{T} \bar{y} \preceq 0$ implies $h_{j} \cdot \bar{y} \leq 0$ for $j=p, \ldots, q$. Also,

$$
h_{j} \cdot \bar{y}=\sum_{i=p}^{q} c_{i}\left(h_{j} \cdot b_{i}\right) \leq 0, \quad j \notin\{p, \ldots, q\} .
$$

This implies $0 \neq \bar{y} \in \mathcal{B} \cap \operatorname{cone}(\mathcal{S})$, a contradiction. Therefore, $M_{p, q}$ has the property that the only solution of the inequalities $c \succeq 0$ and $M_{p, q} c \preceq 0$ is $c=0$. By Theorem 2.2(iv) this implies that $M_{p, q}$ is a non-singular $\mathscr{M}$-matrix.

\subsection{Affine Feedback Transformations}

Assumption 5.1 specifies there is a maximal set of linearly independent vectors in $\mathcal{B}$ available to vertices in $\mathcal{G}$ in terms of the variable $m^{\star}$. Let

$$
\hat{\mathcal{B}}:=\operatorname{span}\left\{b_{1}, \ldots, b_{m^{*}} \mid b_{i} \in \mathcal{B} \cap \mathcal{C}_{i}\right\}
$$

Condition (C3) implies that for all $v_{i} \in \mathcal{G}$,

$$
\mathcal{B} \cap \mathcal{C}_{i} \subset \hat{\mathcal{B}} \cap \mathcal{C}_{i}
$$

We show that $\mathcal{B}$ can be restricted to $\hat{\mathcal{B}}$ without affecting solvability of RCP. Thus, a distinction between $m^{\star}$ and $m=\operatorname{rank}(B)$ is not needed. To that end, suppose we have a simplex $\mathcal{S}$ and a system (3.1) such that Assumption 5.1 holds, $m^{\star}<m$, and the invariance conditions are solvable. We construct an affine feedback transformation

$$
u=K_{1} x+g_{1}+G_{1} w
$$

where $w \in \mathbb{R}^{m}$ is a new exogenous input. This yields a new system

$$
\dot{x}=\left(A+B K_{1}\right) x+\left(a+B g_{1}\right)+B G_{1} w=: \hat{A} x+\hat{a}+\hat{B} w .
$$


The parameters $K_{1}, g_{1}$, and $G_{1}$ are selected so that

$$
\begin{array}{rlrl}
\hat{A} v_{i}+\hat{a} & =0, & i \in I_{\mathcal{G}} \\
\hat{A} v_{i}+\hat{a} & \in \mathcal{C}_{i} \backslash \mathcal{B}, & & i \notin I_{\mathcal{G}} \\
\hat{B} & =\left[\begin{array}{llll}
b_{1} & b_{2} & \cdots & b_{m^{\star}}
\end{array}\right] . &
\end{array}
$$

The columns of $\hat{B}$ are given by $(\mathrm{C} 3)$ and $\operatorname{rank}(\hat{B})=m^{\star}$. Note that when $w=0$, the invariance conditions are satisfied at $v_{i} \notin \mathcal{G}$ and equilibria appear at $v_{i} \in \mathcal{G}$. The following is an immediate consequence.

Lemma 5.2. If the invariance conditions for $\mathcal{S}$ are solvable for (3.1), then they are solvable for the system (5.2).

The next result shows that the proposed state feedback transformation does not affect $\mathcal{O}$.

Lemma 5.3. $\mathcal{O}=\hat{\mathcal{O}}:=\left\{x \in \mathbb{R}^{n}: \hat{A} x+\hat{a} \in \hat{\mathcal{B}}\right\}$.

Proof. Let $v_{i} \in \mathcal{O}$. Then by (5.3), $\hat{A} v_{i}+\hat{a}=0$, so $\hat{A} v_{i}+\hat{a} \in \hat{\mathcal{B}}$. Thus, $v_{i} \in \hat{\mathcal{O}}$. Conversely, let $v_{i} \in \hat{\mathcal{O}}$. Then $\hat{A} v_{i}+\hat{a} \in \hat{\mathcal{B}} \subset \mathcal{B}$. Now $\hat{A} v_{i}+\hat{a}=A v_{i}+a+B K_{1} v_{i}+B g_{1}$, so $A v_{i}+a=\hat{A} v_{i}+\hat{a}-B\left(K_{1} v_{i}+g_{1}\right) \in \mathcal{B}$. Therefore, $v_{i} \in \mathcal{O}$. The result now follows by taking the affine hull of $\left\{v_{1}, \ldots, v_{\kappa+1}\right\}$.

We claim it is no loss of generality to work with the reduced system, assuming one has fixed an appropriate class of controls. First, if we find $w=f(x)$ such that $\mathcal{S} \stackrel{\mathcal{S}}{\longrightarrow} \mathcal{F}_{0}$ for the reduced system, then we have a solution for the original system (3.1) by setting

$$
u=K_{1} x+g_{1}+G_{1} f(x) .
$$

For the converse statement we consider here affine feedbacks.

Proposition 5.1. If there exists $u=K x+g$ such that $\mathcal{S} \stackrel{\mathcal{S}}{\longrightarrow} \mathcal{F}_{0}$ for (3.1), then there exist $u=K_{1} x+g_{1}+G_{1} w$ and $w=K_{2} x+g_{2}$ such that (5.3)-(5.4) hold and $\mathcal{S} \stackrel{\mathcal{S}}{\longrightarrow} \mathcal{F}_{0}$ for (5.2). 
Proof. Let $u_{i}:=K v_{i}+g$ for $v_{i} \in V$. Also fix $G_{1}$ such that $\hat{B}=B G_{1}$. In the first step we show that for each $v_{i} \in V$, there exist $u_{i}^{\prime}$ and $w_{i}$ such that

$$
A v_{i}+a+B u_{i}=A v_{i}+a+B u_{i}^{\prime}+\hat{B} w_{i}
$$

and (5.3)-(5.4) hold. First consider $v_{i} \in \mathcal{G}$. Since $A v_{i}+a \in \mathcal{B}$, there exists $u_{i}^{\prime} \in \mathbb{R}^{m}$ such that

$$
A v_{i}+a+B u_{i}^{\prime}=0 .
$$

Set $q_{i}=u_{i}-u_{i}^{\prime}$. Invoking Theorem 3.3(a) we have

$$
A v_{i}+a+B u_{i}=A v_{i}+a+B\left(u_{i}^{\prime}+q_{i}\right)=B q_{i} \in \mathcal{B} \cap \mathcal{C}_{i} \subset \hat{B} \cap \mathcal{C}_{i} .
$$

This implies there exists $w_{i}$ such that $B q_{i}=\hat{B} w_{i}$. Therefore we obtain

$$
A v_{i}+a+B u_{i}=A v_{i}+a+B u_{i}^{\prime}+\hat{B} w_{i}
$$

with $A v_{i}+a+B u_{i}^{\prime}=0$. Next, consider $v_{i} \notin \mathcal{G}$. Then $A v_{i}+a \notin \mathcal{B}$ and by Theorem 3.3(a), $A v_{i}+a+B u_{i} \in \mathcal{C}_{i} \backslash \mathcal{B}$. This can be written as

$$
A v_{i}+a+B u_{i}=A v_{i}+a+B u_{i}^{\prime}+\hat{B} w_{i}
$$

with $u_{i}^{\prime}:=u_{i}$ and $w_{i}:=0$.

In the second step, the affine feedback $u=K_{1} x+g_{1}$ can be obtained by solving the system of equations

$$
u_{i}^{\prime}=K_{1} v_{i}+g_{1}, \quad i=0, \ldots, n
$$

for unknowns $K_{1}$ and $g_{1}$. See [15]. By construction, (5.3)-(5.4) follow. Similiarly, $w=$ $K_{2} x+g_{2}$ is obtained by solving

$$
w_{i}=K_{2} v_{i}+g_{2}, \quad i=0, \ldots, n
$$

for unknowns $K_{2}$ and $g_{2}$. In the final step, we observe that the right-hand sides of the closed-loop systems (3.1) and (5.2) are identical, so $\mathcal{S} \stackrel{\mathcal{S}}{\longrightarrow} \mathcal{F}_{0}$ for (5.2). 


\subsection{Reach Control Indices}

In this section we adopt the necessary conditions of Section 3.2, we apply the feedback transformation of Section 5.2, and we consider those cases when RCP is not solvable by affine state feedback. The situation is summarized by the following assumptions.

Assumption 5.2. Simplex $\mathcal{S}$ and system (3.1) satisfy the following conditions.

$(\mathrm{R} 1) \mathcal{G}=$ co $\left\{v_{1}, \ldots, v_{\kappa+1}\right\}$, where $m \leq \kappa<n$

$(\mathrm{R} 2) \mathcal{B} \cap \operatorname{cone}(\mathcal{S})=\mathbf{0}$.

(R3) $\mathcal{B}=\operatorname{span}\left\{b_{1}, \ldots, b_{m} \mid b_{i} \in \mathcal{B} \cap \mathcal{C}_{i}\right\}$

$(\mathrm{R} 4) \mathcal{B} \cap \mathcal{C}_{i} \neq \mathbf{0}, \quad i \in I_{\mathcal{G}}$

From condition (R3) we have a preferred set of vectors $\left\{b_{1}, \ldots, b_{m} \mid b_{i} \in \mathcal{B} \cap \mathcal{C}_{i}\right\}$ which span $\mathcal{B}$. Of course there are many possible choices for this basis, but we assume for the following development that these vectors have been fixed. By condition (R1), we have

$$
\kappa+1=m+p
$$

for some $p \geq 1$. Using (R4), if we select any set $\left\{b_{m+1}, \ldots, b_{\kappa+1} \mid b_{i} \in \mathcal{B} \cap \mathcal{C}_{i}\right\}$ and we use $\left\{b_{1}, \ldots, b_{m}\right\}$ as in (R3), then $p$ denotes the number of linearly dependent vectors in the set $\left\{b_{1}, \ldots, b_{\kappa+1}\right\}$. Consider now $\mathcal{B} \cap \mathcal{C}_{m+1}$. It satisfies $b_{m+1} \in \operatorname{span}\left\{b_{1}, \ldots, b_{m}\right\}$ for every $b_{m+1} \in \mathcal{B} \cap \mathcal{C}_{m+1}$. Using (R4), there exists $1 \leq \rho_{1} \leq m$ such that w.l.o.g. (reordering indices $1, \ldots, m)$,

$$
\mathcal{B} \cap \mathcal{C}_{m+1} \subset \operatorname{span}\left\{b_{1}, \ldots, b_{\rho_{1}}\right\}
$$

and $\operatorname{span}\left\{b_{1}, \ldots, b_{\rho_{1}}\right\}$ is the smallest such subspace generated by the basis $\left\{b_{1}, \ldots, b_{m}\right\}$. One can think of $\operatorname{span}\left\{b_{1}, \ldots, b_{\rho_{1}}\right\}$ as a container subspace for the cone $\mathcal{B} \cap \mathcal{C}_{m+1}$. 
This argument can be repeated for each of the remaining cones $\mathcal{B} \cap \mathcal{C}_{i}, \quad i=m+$ $2, \ldots, m+p$ to obtain indices $\pi_{k} \leq \rho_{k}, k=1, \ldots, p$, with $\pi_{1}=1$, such that

$$
\begin{aligned}
\mathcal{B} \cap \mathcal{C}_{m+1} & \subset \operatorname{span}\left\{b_{\pi_{1}}, \ldots, b_{\rho_{1}}\right\} \\
\mathcal{B} \cap \mathcal{C}_{m+2} & \subset \operatorname{span}\left\{b_{\pi_{2}}, \ldots, b_{\rho_{2}}\right\} \\
& \vdots \\
\mathcal{B} \cap \mathcal{C}_{m+p} & \subset \operatorname{span}\left\{b_{\pi_{p}}, \ldots, b_{\rho_{p}}\right\} .
\end{aligned}
$$

Moreover, for the fixed basis $\left\{b_{1}, \ldots, b_{m}\right\}$, these subspaces represent the minimal subspaces that contain the respective cones. This has the interpretation that every basis vector $b_{j}$ in a container subspace contributes to generating at least one vector in the associated cone $\mathcal{B} \cap \mathcal{C}_{k}$. The following result establishes that one can always find a vector in each cone which depends all on the basis vectors of its container subspace.

Lemma 5.4. Suppose Assumption 5.2 and (5.6)-(5.8) hold. For each $k=1, \ldots, p$, there exists $b_{m+k} \in \mathcal{B} \cap \mathcal{C}_{m+k}$ such that

$$
b_{m+k}=c_{\pi_{k}} b_{\pi_{k}}+\cdots+c_{\rho_{k}} b_{\rho_{k}}, \quad c_{i} \neq 0 .
$$

Proof. We consider only the case $k=1$; the other cases follow by reordering indices $k=1, \ldots, p$. Suppose w.l.o.g. that for all $b \in \mathcal{B} \cap \mathcal{C}_{m+1}$,

$$
b=c_{1} b_{1}+\cdots+c_{\rho_{1}-1} b_{\rho_{1}-1} .
$$

That is, no $b \in \mathcal{B} \cap \mathcal{C}_{m+1}$ depends on $b_{\rho_{1}}$. Then $\mathcal{B} \cap \mathcal{C}_{m+1} \subset \operatorname{span}\left\{b_{1}, \ldots, b_{\rho_{1}-1}\right\}$, which contradicts the definition of $\rho_{1}$. Therefore, for each $i \in\left\{1, \ldots, \rho_{1}\right\}$ there exists $\beta_{i} \in$ $\mathcal{B} \cap \mathcal{C}_{m+1}$ such that

$$
\beta_{i}=\mu_{1}^{i} b_{1}+\cdots+\mu_{\rho_{1}}^{i} b_{\rho_{1}}
$$

with $\mu_{i}^{i} \neq 0$. Now we show inductively that $b$ satisfying (5.9) exists. Let $j=1$. Set

$$
b=\beta_{1} \in \mathcal{B} \cap \mathcal{C}_{m+1} .
$$


Then $b$ satisfies $c_{1}:=\mu_{1}^{1} \neq 0$. Next suppose there exists $b \in \mathcal{B} \cap \mathcal{C}_{m+1}$ such that

$$
b=c_{1} b_{1}+\cdots+c_{j} b_{j}+\cdots+c_{\rho_{1}} b_{\rho_{1}}
$$

and $c_{i} \neq 0$, for $i=1, \ldots, j$. Moreover, by reordering indices $j+1, \ldots, \rho_{1}$, it can be assumed that $c_{j+1}=0$ (for if this is not possible, then $b$ satisfies (5.9) and the induction is finished). Let

$$
\bar{c}:=\min _{i \in\{1, \ldots, j\}}\left|c_{i}\right|>0
$$

Consider $\beta_{j+1}=\mu_{1}^{j+1} b_{1}+\cdots+\mu_{\rho_{1}}^{j+1} b_{\rho_{1}}$, with $\mu_{j+1}^{j+1} \neq 0$. We choose $\alpha \in(0,1)$ such that

$$
\alpha\left|\mu_{i}^{j+1}\right|<\bar{c}, \quad i=1, \ldots, j
$$

Now let

$$
b^{\prime}:=b+\alpha \beta_{j+1} \in \mathcal{B} \cap \mathcal{C}_{m+1} .
$$

Then $b^{\prime}=c_{1}^{\prime} b_{1}+\cdots+c_{\rho_{1}}^{\prime} b_{\rho_{1}}$, where, using (5.10),

$$
\begin{aligned}
c_{i}^{\prime} & =c_{i}+\alpha \mu_{i}^{j+1} \neq 0, \quad i=1, \ldots, j \\
c_{j+1}^{\prime} & =\alpha \mu_{j+1}^{j+1} \neq 0 .
\end{aligned}
$$

Therefore $b^{\prime}$ replaces $b$ and the induction step is complete.

The dependency of cones on a limited number of vectors in $\mathcal{B}$ places restrictions on the orientation of those vectors with respect to $\mathcal{S}$.

Lemma 5.5. Suppose Assumption 5.2 and (5.6)-(5.8) hold. For each $k=1, \ldots, p$ and for any $b_{m+k} \in \mathcal{B} \cap \mathcal{C}_{m+k}$, we have

$$
h_{j} \cdot b_{i}=0, \quad i=\pi_{k}, \ldots, \rho_{k}, m+k, \quad j=I \backslash\left\{\pi_{k}, \ldots, \rho_{k}, m+k\right\}
$$

Proof. We consider only the case $k=1$; the other cases follow by reordering indices $k=1, \ldots, p$. Also let $\rho:=\rho_{1}$. Following Lemma 5.4, select $b_{m+1} \in \mathcal{B} \cap \mathcal{C}_{m+1}$ such that

$$
b_{m+1}=c_{1} b_{1}+\cdots+c_{\rho} b_{\rho}, \quad c_{i} \neq 0 .
$$


Define $c:=\left(c_{1}, \ldots, c_{\rho}\right)$. Since $\left\{b_{1}, \ldots, b_{\rho}\right\}$ are linearly independent and $\mathcal{B} \cap \operatorname{cone}(\mathcal{S})=\mathbf{0}$, by Lemma 5.1, $M_{1, \rho}$ is a non-singular $\mathscr{M}$-matrix. Consider the following invariance conditions for $v_{m+1}$ :

$$
H_{1, \rho}^{T} b_{m+1}=H_{1, \rho}^{T} Y_{1, \rho} c=M_{1, \rho} c \preceq 0 .
$$

By Theorem $2.2($ case $(v))$ and the fact that $c_{i} \neq 0$, we obtain $c \prec 0$. Now consider the remaining invariance conditions for $v_{m+1}$ :

$$
h_{j} \cdot b_{m+1}=h_{j} \cdot\left(c_{1} b_{1}+\cdots+c_{\rho} b_{\rho}\right) \leq 0, \quad j=\rho+1, \ldots, m, m+2, \ldots, n .
$$

Every term in the sum is non-negative, since $b_{i} \in \mathcal{B} \cap \mathcal{C}_{i}$ and $c_{i}<0$, and so we obtain

$$
h_{j} \cdot b_{i}=0, \quad i=1, \ldots, \rho, m+1, \quad j=\rho+1, \ldots, m, m+2, \ldots, n .
$$

Using the restrictions placed in $\mathcal{B}$, it can be shown that the container subspaces in (5.6)-(5.8) split into independent subspaces, thus yielding a decomposition of $\mathcal{B}$ with respect to $\mathcal{G}$.

Lemma 5.6. Suppose Assumption 5.2 and (5.6)-(5.8) hold. Then there exists an ordering of indices $\{1, \ldots, m\}$ such that

$$
\rho_{k}<\pi_{k+1}, \quad k=1, \ldots, p-1 .
$$

Proof. We consider only the case $k=1$; the other cases follow by reordering indices. Suppose by way of contradiction that $\rho_{1} \geq \pi_{2}$. Applying Lemma 5.5 we obtain

$$
\begin{aligned}
& h_{j} \cdot b_{i}=0, \quad i=1, \ldots, \rho_{1}, \quad j=\rho_{1}+1, \ldots, m, m+2, \ldots, n \\
& h_{j} \cdot b_{i}=0, \quad i=\pi_{2}, \ldots, \rho_{2}, \quad j=1, \ldots, \pi_{2}-1, \rho_{2}+1, \ldots, m+1, m+3, \ldots,
\end{aligned}
$$

Consider

$$
M_{\pi_{2}, \rho_{1}}=H_{\pi_{2}, \rho_{1}}^{T} Y_{\pi_{2}, \rho_{1}}
$$


where $Y_{\pi_{2}, \rho_{1}}=\left[b_{\pi_{2}} \cdots b_{\rho_{1}}\right]$. Since $\left\{b_{\pi_{2}}, \ldots, b_{\rho_{1}}\right\}$ are linearly independent, by Lemma 5.1, $M_{\pi_{2}, \rho_{1}}$ is a non-singular $\mathscr{M}$-matrix. By Theorem 2.2 (case $\left.(i i i)\right)$ there exists $c^{\prime}=$ $\left(c_{\pi_{2}}^{\prime}, \ldots, c_{\rho_{1}}^{\prime}\right)$ such that $c^{\prime} \preceq 0$ and $M_{\pi_{2}, \rho_{1}} c^{\prime} \prec 0$. Define $\beta:=Y_{\pi_{2}, \rho_{1}} c^{\prime} \neq 0$. The statement $H_{\pi_{2}, \rho_{1}}^{T} \beta=M_{\pi_{2}, \rho_{1}} c^{\prime} \prec 0$ gives

$$
h_{j} \cdot \beta<0, \quad j=\pi_{2}, \ldots, \rho_{1} .
$$

Also, by (5.12)-(5.13)

$$
h_{j} \cdot \beta=h_{j} \cdot\left(c_{\pi_{2}}^{\prime} b_{\pi_{2}}+\cdots+c_{\rho_{1}}^{\prime} b_{\rho_{1}}\right)=0, \quad j=1, \ldots, \pi_{2}-1, \rho_{1}+1, \ldots, n
$$

This implies $\beta \in \mathcal{B} \cap \operatorname{cone}(\mathcal{S})$. By Assumption (R2) we obtain $\beta=0$, a contradiction.

We can show that the decomposition obtained so far extends to all cones $\mathcal{B} \cap \mathcal{C}_{i}$ associated with $\mathcal{G}$. This is based on the observation that the selection of basis vectors $\left\{b_{1}, \ldots, b_{m}\right\}$ is arbitrary. For example $\left\{b_{2}, \ldots, b_{m+1}\right\}$, with $b_{m+1}$ as in Lemma 5.4 , could have been used as a basis for $\mathcal{B}$ and all foregoing results would apply. In recognition of the fact that no special role is played by vertices $v_{m+1}, \ldots, v_{m+p}$, we define a new set of indices which place dependent cones within consecutively numbered container subspaces. To do so, we first reorder indices so that the container subspaces have basis elements which are consecutively numbered:

$$
\left\{b_{1}, \ldots, b_{\rho_{1}}\right\},\left\{b_{\rho_{1}+1}, \ldots, b_{\rho_{2}}\right\}, \ldots,\left\{b_{\rho_{p-1}+1}, \ldots, b_{\rho_{p}}\right\} .
$$

Now adjoint to these lists the vectors $b_{m+k}, k=1, \ldots, p$, obtained from Lemma 5.4, to get the new lists

$$
\left\{b_{1}, \ldots, b_{\rho_{1}}, b_{m+1}\right\},\left\{b_{\rho_{1}+1}, \ldots, b_{\rho_{2}}, b_{m+2}\right\}, \ldots,\left\{b_{\rho_{p-1}+1}, \ldots, b_{\rho_{p}}, b_{m+p}\right\} .
$$

Finally, define new indices so that also these lists are consecutive. That is, there exist indices $r_{1}, \ldots, r_{p}$ such that the previous list becomes

$$
\left\{b_{1}, \ldots, b_{r_{1}}\right\},\left\{b_{r_{1}+1}, \ldots, b_{r_{1}+r_{2}}\right\}, \ldots,\left\{b_{r_{1}+\cdots+r_{p-1}+1}, \ldots, b_{r}\right\}
$$


where

$$
r:=r_{1}+\cdots+r_{p}
$$

Using (5.6)-(5.8) and Lemma 5.6 with our new numbering, we get

$$
\begin{aligned}
\mathcal{B} \cap \mathcal{C}_{r_{1}} & \subset \mathcal{B}_{1}:=\operatorname{span}\left\{b_{1}, \ldots, b_{r_{1}}\right\} \\
\mathcal{B} \cap \mathcal{C}_{r_{1}+r_{2}} & \subset \mathcal{B}_{2}:=\operatorname{span}\left\{b_{r_{1}+1}, \ldots, b_{r_{1}+r_{2}}\right\} \\
& \vdots \\
\mathcal{B} \cap \mathcal{C}_{r_{1}+\cdots+r_{p}} & \subset \mathcal{B}_{p}:=\operatorname{span}\left\{b_{r_{1}+\cdots+r_{p-1}+1}, \ldots, b_{r}\right\} .
\end{aligned}
$$

Theorem 5.1. Suppose Assumption 5.2 holds. Then there exist integers $r_{1}, \ldots, r_{p} \geq 0$ and a decomposition of $\mathcal{B}$ into $p$ subsets such that

$$
\begin{array}{rlrl}
\mathcal{B} \cap \mathcal{C}_{i} \subset \operatorname{span}\left\{b_{1}, \ldots, b_{r_{1}}\right\}, & i=1, \ldots, r_{1}, \\
\mathcal{B} \cap \mathcal{C}_{i} \subset \operatorname{span}\left\{b_{r_{1}+1}, \ldots, b_{r_{1}+r_{2}}\right\}, & i=r_{1}+1, \ldots, r_{1}+r_{2} \\
\vdots & & \vdots \\
\mathcal{B} \cap \mathcal{C}_{i} \subset \operatorname{span}\left\{b_{r_{1}+\cdots+r_{p-1}+1}, \ldots, b_{r}\right\}, & i=r_{1}+\cdots+r_{p-1}+1, \ldots, r .
\end{array}
$$

The proof relies on a preliminary lemma proved only for $k=1$.

Lemma 5.7. Suppose Assumption 5.2 and (5.14)-(5.16) hold. There does not exist $\beta \in \operatorname{span}\left\{b_{r_{1}+1}, \ldots, b_{m+p}\right\}, \beta \neq 0$, such that $\left\{b_{1}, \ldots, b_{r_{1}-1}, \beta\right\}$ are linearly independent and

$$
h_{j} \cdot \beta \leq 0, \quad j=r_{1}+1, \ldots, n .
$$

Proof. Since $\left\{b_{1}, \ldots, b_{r_{1}-1}\right\}$ are linearly independent and $\mathcal{B} \cap \operatorname{cone}(\mathcal{S})=\mathbf{0}$, by Lemma 5.1, $M_{1, r_{1}-1}$ is a non-singular $\mathscr{M}$-matrix. Applying Theorem 2.2 (case $(i i i)$ ) there exists $c^{\prime}=\left(c_{1}^{\prime}, \ldots, c_{r_{1}-1}^{\prime}\right)$ such that $c^{\prime} \preceq 0$ and $M_{1, r_{1}-1} c^{\prime} \prec 0$. Define $b_{r_{1}}^{\prime}:=Y_{1, r_{1}-1} c^{\prime}$. The vector $H_{1, n}^{T} b_{r_{1}}^{\prime} \in \mathbb{R}^{n}$ has the following sign pattern:

$$
(-, \ldots,-, *, 0, \ldots, 0)
$$


where the $*$ appears in the $\left(r_{1}\right)$ th component and the zero components are due to Lemma 5.5. In particular $b_{r_{1}}^{\prime} \in \mathcal{B} \cap \mathcal{C}_{r_{1}}$ and the first $r_{1}-1$ invariance conditions are strictly negative. Now suppose we find a non-zero vector $\beta \in \operatorname{span}\left\{b_{r_{1}+1}, \ldots, b_{m+p}\right\}$ such that (5.20) holds and $\left\{b_{1}, \ldots, b_{r_{1}-1}, \beta\right\}$ are linearly independent. Then for $\alpha>0$ we can form

$$
b_{r_{1}}^{\prime \prime}:=b_{r_{1}}^{\prime}+\alpha \beta .
$$

Using (5.21) and (5.20), $\alpha$ can be selected sufficiently small so that $h_{j} \cdot b_{r_{1}}^{\prime \prime} \leq 0$ for all $j=1, \ldots, r_{1}-1, r_{1}+1, \ldots, n$. That is, $b_{r_{1}}^{\prime \prime} \in \mathcal{B} \cap \mathcal{C}_{r_{1}}$. Moreover, with $\beta \neq 0$,

$$
\left\{b_{1}, \ldots, b_{r_{1}-1}, b_{r_{1}}^{\prime \prime}\right\}
$$

is a linearly independent set. This contradicts (5.14).

Proof of Theorem 5.1. We consider only $k=1$. We must show $\mathcal{B} \cap \mathcal{C}_{i} \subset \operatorname{span}\left\{b_{1}, \ldots, b_{r_{1}}\right\}$, for $i=1, \ldots, r_{1}$. (By (5.14) we already have $\left.\mathcal{B} \cap \mathcal{C}_{r_{1}} \subset \operatorname{span}\left\{b_{1}, \ldots, b_{r_{1}-1}\right\}\right)$. Consider any $i \in\left\{1, \ldots, r_{1}\right\}$ and any $\beta_{i} \in \mathcal{B} \cap \mathcal{C}_{i}$ such that

$$
\beta_{i}=c_{1} b_{1}+\cdots+c_{r_{1}} b_{r_{1}}+\beta
$$

where $c_{i} \in \mathbb{R}$ and $\beta \in \operatorname{span}\left\{b_{r_{1}+1}, \ldots, b_{m+p}\right\}$. W.l.o.g. assume that $\beta$ is independent of $\left\{b_{1}, \ldots, b_{r_{1}-1}\right\}$ (otherwise the $c_{i}$ 's can be redefined so that $\beta=0$ and the result is immediately obtained). From the invariance conditions associated with $v_{i}$ and by Lemma 5.5, we have

$$
h_{j} \cdot \beta_{i}=h_{j} \cdot\left(c_{1} b_{1}+\cdots+c_{r_{1}} b_{r_{1}}+\beta\right)=h_{j} \cdot \beta \leq 0, \quad j=r_{1}+1, \ldots, n .
$$

By Lemma 5.7, $\beta=0$. Hence, for any $i \in\left\{1, \ldots, r_{1}\right\}$ and any $\beta_{i} \in \mathcal{B} \cap \mathcal{C}_{i}, \beta_{i} \in$ $\operatorname{span}\left\{b_{1}, \ldots, b_{r_{1}}\right\}$, as desired.

The lists in (5.17)-(5.19) have the property that any vector in a list on the right is dependent on all the other vectors in its list. Also, if any vector is removed from a list, the remaining vectors are linearly independent. In particular, the $k$ th list has $r_{k}-1$ linearly 
independent vectors of $\mathcal{B}$. We can say that $\mathcal{B}$ has been decomposed into $p$ independent cycles of dependency. Also, because of (R4), $p \leq m$. Thus, in order for (R4) to hold it is necessary that

$$
m \geq \frac{\kappa+1}{2}
$$

This condition is interpreted to say that RCP is only solvable if there are sufficient inputs. Since each of the $p$ lists comprises $r_{k}-1$ independent vectors in $\mathcal{B}$ and there are a total of $m$ such vectors, we deduce that

$$
r-p \leq m
$$

Definition 5.1. The integers $\left\{r_{1}, \ldots, r_{p}\right\}$ are called the reach control indices of system (3.1) with respect to simplex $\mathcal{S}$.

From the previous developments, it is straightforward to show the following.

Proposition 5.2. The reach control indices are: (i) invariant under affine feedback transformations $u=K x+g+w$; (ii) unique up to ordering of indices; and (iii) independent of choice of maximal linearly independent set in $\left\{b_{1}, \ldots, b_{\kappa+1} \mid b_{i} \in \mathcal{B} \cap \mathcal{C}_{i}\right\}$.

\subsection{Existence of Equilibria}

In this section we explore cases when equilibria appear on $\mathcal{G}$ when an assignment of a continuous state feedback $y(x)$ is made on $\mathcal{S}$, so that RCP is not solvable by continuous state feedback. Let $u(x)$ be a continuous state feedback defined on $\mathcal{S}$. We restrict our attention to such controls that yield unique solutions on $\mathcal{S}$ and which satisfy the invariance conditions $(3.6)$ on $\mathcal{S}$. Define the closed-loop system

$$
\dot{x}=A x+B u(x)+a=: y(x) .
$$


Our main tool for isolating closed-loop equilibria when using continuous state feedback is the reach control indices. To that end,, define for $k=1, \ldots, p$

$$
\begin{aligned}
m_{k} & :=r_{1}+\cdots+r_{k-1}+1 \\
\widehat{\mathcal{S}}_{k} & :=\operatorname{co}\left\{v_{m_{k}}, \ldots, v_{m_{k}+r_{k}-1}\right\}
\end{aligned}
$$

The following lemma gives the direct consequence of Lemma 5.7 in the case of continuous state feedback.

Proposition 5.3. Suppose Assumption 5.2 holds. Let $u(x)$ be a continuous state feedback satisfying the invariance conditions (3.6). Then for each $k=1, \ldots, p$ and $x \in \widehat{\mathcal{S}}_{k}$,

$$
h_{j} \cdot y(x)=0, \quad j \in I \backslash\left\{m_{k}, \ldots, m_{k}+r_{k}-1\right\} .
$$

Proof. We prove the result only for $k=1$. Let $y(x)=A x+B u(x)+a$ be the closed-loop vector field on $\mathcal{S}$ satisfying the invariance conditions (3.6). For $x \in \widehat{\mathcal{S}}_{1}$, we have

$$
y(x)=c_{1}(x) b_{1}+\cdots+c_{r_{1}}(x) b_{r_{1}}+\beta(x),
$$

where $\beta(x) \in \operatorname{span}\left\{b_{r_{1}+1}, \ldots, b_{m+p}\right\}$. We may assume w.l.o.g. (as in the proof of Theorem 5.1) that $\beta(x)$ is independent of $\left\{b_{1}, \ldots, b_{r_{1}}\right\}$. From (3.6) we know

$$
h_{j} \cdot y(x) \leq 0, \quad j=r_{1}+1, \ldots, n
$$

Using (5.24) and Lemma 5.5, these conditions become

$$
h_{j} \cdot \beta(x) \leq 0, \quad j=r_{1}+1, \ldots, n .
$$

By Lemma 5.7, this implies $\beta(x)=0$. Therefore, for each $x \in \widehat{\mathcal{S}}_{1}$,

$$
h_{j} \cdot y(x)=0, \quad j=r_{1}+1, \ldots, n .
$$


Remark 5.1.1. Proposition 5.3 has the following intuitive meaning. For simplicity suppose $v_{0}=0$. We know from the geometry of the simplex (see Lemma 2.1) that the state space can be decomposed as follows:

$$
\mathbb{R}^{n}=\operatorname{aff}\left\{v_{0}, \ldots, v_{r_{1}}\right\} \oplus \operatorname{span}\left\{h_{r_{1}+1}, \ldots, h_{n}\right\} \simeq \mathbb{R}^{r_{1}} \oplus \mathbb{R}^{n-r_{1}}
$$

Therefore, Proposition 5.3 says that

$$
\operatorname{span}\left\{b_{1}, \ldots, b_{r_{1}}\right\} \subset \text { aff }\left\{v_{0}, \ldots, v_{r_{1}}\right\}
$$

Moreover, for all $x \in \widehat{\mathcal{S}}_{1}$,

$$
y(x) \in \operatorname{span}\left\{b_{1}, \ldots, b_{r_{1}}\right\} .
$$

Geometrically, $\widehat{\mathcal{S}}_{1}$ lies in aff $\left\{v_{0}, \ldots, v_{r_{1}}\right\}$, an $r_{1}$-dimensional affine space in $\mathbb{R}^{n}$, and it is itself a $\left(r_{1}-1\right)$-dimensional simplex in this space. Meanwhile, $\mathcal{B}$ provides to $\widehat{\mathcal{S}}_{1}$ only $r_{1}-1$ usable directions (which also lie in aff $\left\{v_{0}, \ldots, v_{r_{1}}\right\}$ ) to resolve all its invariance conditions. We will see that this is not enough to establish a flow condition on $\widehat{\mathcal{S}}_{1}$.

Consider again Figure 4.2 in which $\mathcal{B} \cap \operatorname{cone}(\mathcal{S})=\mathbf{0}$. We have that $n=3, m=1$, and $\kappa=1$. Thus, $\mathcal{G}=\overline{v_{1} v_{2}}$. Also, $\mathcal{B}=\operatorname{span}\left\{b_{1} \mid b_{1} \in \mathcal{B} \cap \mathcal{C}_{1}\right\}$. We can see that in order not to have any $\mathcal{B} \cap \mathcal{C}_{i}=\{0\}$, for $i=1,2$, it must be that $\mathcal{B} \subset$ aff $\left\{v_{0}, v_{1}, v_{2}\right\}$, which is the affine (linear) space that contains the convex hull of $\mathcal{G}$ and $v_{0}$. Notice that this space is equivalently definable by $\left\{y \mid h_{3} \cdot y=0\right\}$. Proposition 5.3 then says that all velocity vectors available to $\mathcal{G}$ lie in aff $\left\{v_{0}, v_{1}, v_{2}\right\}$.

Proposition 5.3 describes the fundamental geometric property that forces the existence of an equilibrium. The proof that an equilibrium exists is based on Sperner's Lemma. See Section 2.7.1.

Theorem 5.2. Suppose Assumption 5.2 holds. Let $u(x)$ be a continuous state feedback satisfying the invariance conditions (3.6). Then each $\widehat{\mathcal{S}}_{k}$ contains an equilibrium of the closed-loop system. 
Proof. We consider only $k=1$. Let $\widehat{I}_{1}:=\left\{1, \ldots, r_{1}\right\}$ and define the closed-loop vector field

$$
y(x):=A x+B u(x)+a, \quad x \in \mathcal{S}
$$

Now we show how to obtain a proper labeling of $\widehat{\mathcal{S}}_{1}$, as defined in Section 2.7.1. We begin by defining the sets

$$
\mathcal{Q}_{i}:=\left\{x \in \widehat{\mathcal{S}}_{1} \mid h_{i} \cdot y(x)>0\right\}, \quad i \in \widehat{I}_{1}
$$

Observe that $v_{i} \in \mathcal{Q}_{i}$ and $v_{i} \notin \mathcal{Q}_{j}, i, j \in \widehat{I_{1}}, i \neq j$, for otherwise, we would have $y\left(v_{i}\right) \in \mathcal{B} \cap \operatorname{cone}(\mathcal{S})$ which either contradicts that $\mathcal{B} \cap \operatorname{cone}(\mathcal{S})=\mathbf{0}$ or implies $y\left(v_{i}\right)$ is an equilibrium. Therefore, either the proof concludes with an equilibrium on a vertex of $\widehat{\mathcal{S}}_{1}$, or we can infer that inclusion in a set $\mathcal{Q}_{i}$ provides a distinct label for the vertices $v_{i} \in \widehat{\mathcal{S}}_{1}$. This satisfies (L1) of a proper labeling of $\widehat{\mathcal{S}}_{1}$. Next, let $\mathbb{T}$ be any triangulation of $\widehat{\mathcal{S}}_{1}$ and consider a vertex $v$ of $\mathbb{T}$ which is not a vertex of $\widehat{\mathcal{S}}_{1}$ and lies in $\partial \widehat{\mathcal{S}}_{1}$. W.l.o.g. let $v \in$ co $\left\{v_{1}, \ldots, v_{l}\right\}$ for some $2 \leq l \leq r_{1}-1$. Then it must be that $v \in \mathcal{Q}_{k}$ for some $1 \leq k \leq l$, by the same reasoning that otherwise $y(v) \in \mathcal{B} \cap \operatorname{cone}(\mathcal{S})$. Clearly this labeling of $v$ satisfies the second condition (L2) for a proper labeling. Finally, for vertices $v$ of $\mathbb{T}$ in the interior of $\widehat{\mathcal{S}}_{1}$, any label $\mathcal{Q}_{i}$ such that $h_{i} \cdot y(v)>0$ can be used (at least one such exists because if all $h_{i} \cdot y(v) \leq 0, i \in \widehat{I}_{1}$, it implies $h_{i} \cdot y(v) \leq 0$ for all $i=1, \ldots, n$ or $y(v) \in \mathcal{B} \cap \operatorname{cone}(\mathcal{S}))$.

Now for each $\alpha>0, \alpha \in \mathbb{Z}$, define a triangulation $\mathbb{T}^{\alpha}$ of $\widehat{\mathcal{S}}_{1}$ such that each simplex of $\mathbb{T}^{k}$ has diameter $\frac{1}{\alpha}$. Apply Sperner's lemma for each $\mathbb{T}^{\alpha}$ to obtain a distinguished simplex co $\left\{v_{1}^{\alpha}, \ldots, v_{r_{1}}^{\alpha}\right\}$ and its baricenter $x^{\alpha}$. The set $\left\{x^{\alpha}\right\}$ defines a bounded sequence in $\widehat{\mathcal{S}}_{1}$ which has a convergent subsequence, again denoted $\left\{x^{\alpha}\right\}$. We have $\lim _{\alpha \rightarrow \infty} x^{\alpha}=\bar{x} \in \widehat{\mathcal{S}}_{1}$, since $\widehat{\mathcal{S}}_{1}$ is closed. Also, by construction $v_{i}^{\alpha} \rightarrow \bar{x}, i \in \widehat{I}_{1}$. By Sperner's lemma we know that $h_{i} \cdot y\left(v_{i}^{\alpha}\right)>0, i \in \widehat{I}_{1}$, so by continuity of $y(x)$ this implies $h_{i} \cdot y(\bar{x}) \geq 0, i \in \widehat{I}_{1}$. Combined with $(5.23)$, we obtain that $-y(\bar{x}) \in \mathcal{B} \cap \operatorname{cone}(\mathcal{S})=\mathbf{0}$, which implies $\bar{x} \in \widehat{\mathcal{S}}_{1}$ is an equilibrium of the closed-loop system $\dot{x}=y(x)$. 


\subsection{Continuous State Feedback}

The next result resolves the boundary between continuous state feedback and affine feedback.

Theorem 5.3. Suppose Assumption 4.1 holds. Then the following statements are equivalent:

(1) $\mathcal{S} \stackrel{\mathcal{S}}{\longrightarrow} \mathcal{F}_{0}$ by affine feedback.

(2) $\mathcal{S} \stackrel{\mathcal{S}}{\longrightarrow} \mathcal{F}_{0}$ by continuous state feedback.

Proof. (1) $\Longrightarrow(2)$ is obvious.

$(2) \Longrightarrow(1)$ Suppose there exists a continuous state feedback $u(x)$ such that the closed loop system (5.22) has a unique solution for each initial condition in $\mathcal{S}$ and RCP is solved using $u(x)$. By Theorem 3.1 the invariance conditions (3.6) are solvable, implying (3.5) are solvable. Suppose $\mathcal{G}=\emptyset$. Then by Theorem $4.1, \mathcal{S} \stackrel{\mathcal{S}}{\longrightarrow} \mathcal{F}_{0}$ by affine feedback. Suppose $\mathcal{G} \neq \emptyset$. Also, suppose $\mathcal{B} \cap \operatorname{cone}(\mathcal{S}) \neq \mathbf{0}$. Then by Theorem $4.2, \mathcal{S} \stackrel{\mathcal{S}}{\longrightarrow} \mathcal{F}_{0}$ by affine feedback. Instead suppose $\mathcal{G} \neq \emptyset$ and $\mathcal{B} \cap \operatorname{cone}(\mathcal{S})=\mathbf{0}$. Suppose $v_{0} \in \mathcal{G}$. Then by Proposition 5.1, the closed-loop system has an equilibrium point $v_{0} \in \mathcal{S}$, a contradiction. Instead suppose $v_{0} \notin \mathcal{G}$ and w.l.o.g. $\mathcal{G}=$ co $\left\{v_{1}, \ldots, v_{\kappa+1}\right\}$, with $0 \leq \kappa<n$. Suppose there does not exist a linearly independent set $\left\{b_{i} \in \mathcal{B} \cap \mathcal{C}_{i} \mid i \in I_{\mathcal{G}}\right\}$. Then by Theorem 5.2 the closed-loop system has an equilibrium point $x_{0} \in \mathcal{S}$, a contradiction. Instead suppose there does exist a linearly independent set $\left\{b_{i} \in \mathcal{B} \cap \mathcal{C}_{i} \mid i \in I_{\mathcal{G}}\right\}$. Then by Theorem 4.3, $\mathcal{S} \stackrel{\mathcal{S}}{\longrightarrow} \mathcal{F}_{0}$ by affine feedback.

The following result provides necessary and sufficient conditions for solvability of RCP by affine feedback.

Theorem 5.4. Suppose Assumption 4.1 holds. $\mathcal{S} \stackrel{\mathcal{S}}{\longrightarrow} \mathcal{F}_{0}$ by affine feedback if and only if the invariance conditions are solvable and one of the following conditions holds: 
(i) $\mathcal{G}=\emptyset$.

(ii) $\mathcal{B} \cap \operatorname{cone}(\mathcal{S})=\mathbf{0}$.

(iii) $\mathcal{G}=\operatorname{co}\left\{v_{1}, \ldots, v_{\kappa+1}\right\}$, with $0 \leq \kappa<n$ and there exists a linearly independent set $\left\{b_{i} \in \mathcal{B} \cap \mathcal{C}_{i} \mid i \in I_{\mathcal{G}}\right\}$. 


\section{Chapter 6}

\section{Reach Control and Piecewise Affine Feedback}

In Chapter 5 it was shown that affine feedback and continuous state feedback are equivalent from the point of view of solvability of the reach control problem. The approach is based, fundamentally, on fixed point theory. The latter allows to deduce that continuous state feedbacks always generate closed-loop equilibria inside the simplex when affine feedbacks do. In this chapter, we depart from these findings and using the reach control indices, we obtain a subdivision of the simplex and an associated piecewise affine feedback to solve RCP in those cases when the problem is not solvable by continuous state feedback. It is shown that RCP is solvable by piecewise affine feedback if it is solvable by open-loop controls.

\subsection{RCP Revisited}

We begin our study of RCP by discontinuous feedback by formulating a slightly stronger version of RCP, which eliminates pathological solutions using discontinuous feedbacks. ${ }^{1}$

\footnotetext{
${ }^{1}$ We suspect this revised version of RCP is not needed, but further work is required to prove that pathologies do not arise with the former problem statement.
} 
Problem 6.1 (Reach Control Problem (RCP)). Consider system (3.1) defined on $\mathcal{S}$. Find a feedback control $u(x)$ such that:

(i) For every $x_{0} \in \mathcal{S}$ there exist $T \geq 0$ such that $\phi_{u}\left(t, x_{0}\right) \in \mathcal{S}$ for all $t \in[0, T]$, and $\phi_{u}\left(T, x_{0}\right) \in \mathcal{F}_{0}$.

(ii) There exists $\varepsilon>0$ such that for all $x \in \mathcal{S}, \quad\|A x+a+B u(x)\|>\varepsilon$.

(iii) Feedback $u(x)$ satisfies the invariance conditions $(3.6)$ on $\mathcal{F}_{0}$.

The new condition is condition (iii), and it is placed for practical reasons to avoid tra-

jectories "spraying out" outside of $\mathcal{S}$ at $\mathcal{F}_{0}$. Importantly, if $\mathcal{S} \stackrel{\mathcal{S}}{\longrightarrow} \mathcal{F}_{0}$ by continuous state feedback in the sense of conditions (i) and (ii) of RCP, then (iii) automatically follows. Therefore, the new condition has a bearing only on solutions of RCP by discontinuous (and open-loop) controls.

\subsection{Piecewise Affine Feedback}

The relevance of the reach control indices is that they catalog the degeneracies (caused by insufficient inputs) that lead to the appearance of equilibria in $\mathcal{S}$ whenever $p \geq 1$ and continuous state feedback is applied. Thus, any control method which overcomes the limits of continuous state feedback must confront this degeneracy and will necessarily draw upon the degrees of freedom in $\mathcal{B}$ provided to $\mathcal{G}$ which are inscribed by the indices. In this section we investigate the extent to which piecewise affine feedback can solve $\mathrm{RCP}$, in cases when continuous state feedback cannot. We construct a triangulation of the simplex $\mathcal{S}$ comprised of sub-simplices such that a sub-RCP is solvable for each sub-simplex. We make the following standing assumptions.

Assumption 6.1. Simplex $\mathcal{S}$ and system (3.1) satisfy the following conditions.

$(\mathrm{P} 1) \mathcal{G}=$ co $\left\{v_{1}, \ldots, v_{\kappa+1}\right\}$, where $m \leq \kappa<n$. 
$(\mathrm{P} 2) \mathcal{B} \cap \operatorname{cone}(\mathcal{S})=\mathbf{0}$.

(P3) $\mathcal{B}=\operatorname{span}\left\{b_{1}, \ldots, b_{m} \mid b_{i} \in \mathcal{B} \cap \mathcal{C}_{i}\right\}$

(P4) $\mathcal{B} \cap \mathcal{C}_{i} \neq \mathbf{0}, \quad i \in I_{\mathcal{G}}$

(P5) $\exists\left\{r_{1}, \ldots, r_{p}\right\}$ such that (5.17)-(5.19) hold.

(P6) $\mathcal{B}_{k} \not \subset \mathcal{F}_{0}, \quad k=1, \ldots, p$.

Conditions (P1)-(P3) define the problem setup. The necessity of (P4) is proved in Theorem 3.2, (P5) is proved in Theorem 5.1, and the necessity of (P6) is proved next.

Lemma 6.1. If $\mathcal{S} \stackrel{\mathcal{S}}{\longrightarrow} \mathcal{F}_{0}$ by open-loop controls, then $\mathcal{B}_{k} \not \subset \mathcal{F}_{0}$ for each $k=1, \ldots, p$.

Proof. We consider only $k=1$. Define

$$
\hat{\mathcal{F}}_{0}:=\operatorname{co}\left\{v_{1}, \ldots, v_{r_{1}}\right\} .
$$

By condition (iii) of RCP, for any open-loop control values $\left\{u_{x}\right\}$ solving RCP,

$$
h_{j} \cdot\left(A x+B u_{x}+a\right) \leq 0, \quad x \in \mathcal{F}_{j} .
$$

By the definition of $\mathcal{B}_{1}$ and by the same proof as in Lemma 5.3, we also have that

$$
h_{j} \cdot\left(A x+B u_{x}+a\right)=0, \quad j=r_{1}+1, \ldots, n .
$$

Suppose by way of contradiction that $\mathcal{B}_{1} \subset \mathcal{F}_{0}$. Then

$$
h_{0} \cdot\left(A x+B u_{x}+a\right)=0 .
$$

Now we observe that for any $x \in \mathcal{S}$

$$
T_{\hat{\mathcal{F}}_{0}}(x)=\left\{y \in \mathbb{R}^{n} \mid h_{j} \cdot y=0, h_{l} \cdot y \leq 0, j=0, r_{1}+1, \ldots, n, x \in \mathcal{F}_{l}\right\}
$$

We conclude that $A x+B u_{x}+a \in T_{\hat{\mathcal{F}}_{0}}(x)$, for all $x \in \hat{\mathcal{F}}_{0}$. By Theorem 3.8 of [11], this implies $\hat{\mathcal{F}}_{0}$ is a strongly invariant set, a contradiction to the statement that $\mathcal{S} \stackrel{\mathcal{S}}{\longrightarrow} \mathcal{F}_{0}$ by open-loop controls. 
Definition 6.1. Given a state feedback $u(x)$, we say $u$ is a piecewise affine feedback if there exists a triangulation $\mathbb{T}$ of $\mathcal{S}$ such that for each (full-dimensional) $\mathcal{S}_{i} \in \mathbb{T}$, there exist $K_{i} \in \mathbb{R}^{m \times n}$ and $g_{i} \in \mathbb{R}^{m}$ such that $u(x)=K_{i} x+g_{i}, x \in \mathcal{S}_{i}$.

We now explain in general terms an inductive procedure for subdividing $\mathcal{S}$ in order that RCP can be solved by piecewise affine feedback. First, in Lemma 6.2 we show that because of condition (P6), each subsimplex $\widehat{\mathcal{S}}_{k}$, for $k=1, \ldots, p$, has a vertex (among $\left.\left\{v_{m_{k}}, \ldots, v_{m_{k}+r_{k}-1}\right\}\right)$ with $b_{i} \in \mathcal{B} \cap \mathcal{C}_{i}$ pointing out of $\mathcal{S}$. By convention, we reorder indices so this vertex is the first one in each list $\left\{v_{m_{k}}, \ldots, v_{m_{k}+r_{k}-1}\right\}$. We make a subdivision of $\mathcal{S}$ by placing a new vertex $v^{\prime}$ along the edge $\left(v_{0}, v_{m_{k}}\right)$. In particular, at the first iteration we would have $v^{\prime} \in\left(v_{0}, v_{1}\right)$, and we form two sub-simplices $\mathcal{S}_{1}$ and $\mathcal{S}^{\prime}$ as in Figure 6.1. Lemma 6.4 shows that because $b_{m_{k}} \in \mathcal{B} \cap \mathcal{C}_{m_{k}}$ points out of $\mathcal{S}$ at $v_{m_{k}}$ and because the invariance conditions for $\mathcal{S}$ are solvable at $v_{0}$, a convexity argument gives that $v^{\prime}$ can be placed along $\left(v_{0}, v_{m_{k}}\right)$ so that $\mathcal{B} \cap \operatorname{cone}\left(\mathcal{S}_{1}\right) \neq 0$. Then in Lemma 6.5 one applies Theorem 4.2 to obtain that RCP is solved for $\mathcal{S}_{1}$. Essentially $\mathcal{S}_{1}$ can be removed from further consideration, and the induction step is repeated with $\mathcal{S}$ replaced by the remainder $\mathcal{S}^{\prime}$. To guarantee that the induction is sound, one must show that $\mathcal{S}^{\prime}$ inherits the relevant properties of $\mathcal{S}$, especially condition $(\mathrm{P} 6)$. This is done in Lemma 6.6.

Lemma 6.2. Suppose Assumption 6.1 holds. Then w.l.o.g. (by reordering indices)

$$
h_{0} \cdot b_{m_{k}}>0, \quad k=1, \ldots, p .
$$

Proof. We prove the result for $k=1$. The other cases follow by reordering indices. If for any $j \in\left\{1, \ldots, r_{1}\right\}, h_{0} \cdot b_{j}>0$, then the proof is finished. Instead suppose that for all $i \in\left\{1, \ldots, r_{1}\right\}, h_{0} \cdot b_{i} \leq 0$. Using (P6) and by reordering the indices $1, \ldots, r_{1}$, assume $h_{0} \cdot b_{r_{1}}<0$. By Lemma 5.4 there exists $b_{1} \in \mathcal{B} \cap \mathcal{C}_{1}$ such that

$$
b_{1}=c_{2} b_{2}+\cdots+c_{r_{1}} b_{r_{1}}, \quad c_{i} \neq 0
$$

and $\left\{b_{2}, \ldots, b_{r_{1}}\right\}$ are linearly independent. Let $c:=\left(c_{2}, \ldots, c_{r_{1}}\right)$. Since $b_{1} \in \mathcal{C}_{1}$, it satisfies 


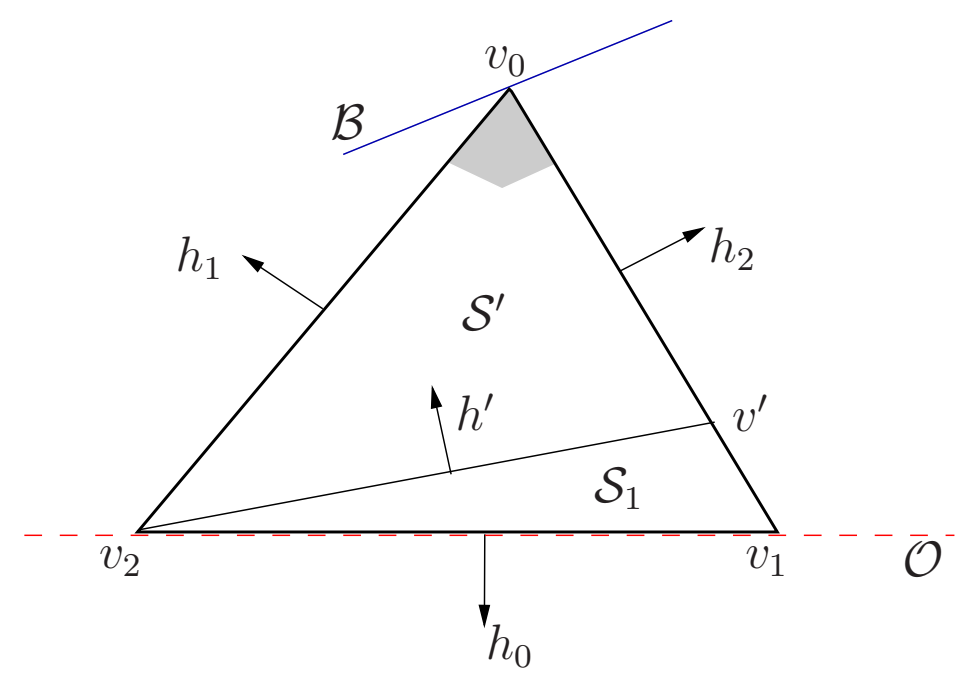

Figure 6.1: Subdivision into two simplices $\mathcal{S}^{\prime}$ and $\mathcal{S}_{1}$.

the invariance conditions:

$$
H_{2, r_{1}}^{T} b_{1}=H_{2, r_{1}}^{T} Y_{2, r_{1}} c=M_{2, r_{1}} c \preceq 0 .
$$

By (P2) and Lemma 5.1, $M_{2, r_{1}}$ is a non-singular $\mathscr{M}$-matrix. By Theorem 2.3 (case $N_{39}$ ) of [4], this implies $c \preceq 0$. Since $c_{i} \neq 0$, we have moreover $c \prec 0$. Thus we obtain

$$
h_{0} \cdot b_{1}=h_{0} \cdot\left(c_{2} b_{2}+\cdots+c_{r_{1}} b_{r_{1}}\right)>0 .
$$

Following Lemma 6.2, suppose that $b_{1}$ satisfies $h_{0} \cdot b_{1}>0$. We consider any point $v^{\prime}$ in the open segment $\left(v_{0}, v_{1}\right)$. That is, let $\lambda \in(0,1)$ and define

$$
v^{\prime}=\lambda v_{1}+(1-\lambda) v_{0} .
$$

Now define the following sub-simplices of $\mathcal{S}$ :

$$
\begin{aligned}
& \mathcal{S}^{\prime}=\operatorname{co}\left\{v_{0}, v^{\prime}, v_{2}, \ldots, v_{n}\right\} \\
& \mathcal{S}_{1}=\operatorname{co}\left\{v^{\prime}, v_{1}, v_{2}, \ldots, v_{n}\right\} .
\end{aligned}
$$

Also define the new exit facet for $\mathcal{S}^{\prime}$ by

$$
\mathcal{F}_{0}^{\prime}:=\operatorname{co}\left\{v^{\prime}, v_{2}, \ldots, v_{n}\right\} .
$$


See Figure 6.1. The following lemma provides a formula for the normal vector $h^{\prime}$ of $\mathcal{F}_{0}^{\prime}$.

Lemma 6.3. Suppose $h_{0}=-\gamma_{1} h_{1}-\ldots-\gamma_{n} h_{n}$ with $\gamma_{i}>0$. Then the normal vector to $\mathcal{F}_{0}^{\prime}$ pointing out of $\mathcal{S}_{1}$ is

$$
h^{\prime}=\gamma_{1} h_{1}+\lambda \sum_{j=2}^{n} \gamma_{j} h_{j} .
$$

Proof. We show that for every $i \in\{2, \ldots, n\}, h^{\prime} \cdot\left(v^{\prime}-v_{i}\right)=0$. From (6.1) we have $v^{\prime}-v_{i}=\lambda\left(v_{1}-v_{0}\right)-\left(v_{i}-v_{0}\right)$. Using (6.2) we have

$$
h^{\prime} \cdot\left(v^{\prime}-v_{i}\right)=\left(\gamma_{1} h_{1}+\lambda \sum_{j=2}^{n} \gamma_{j} h_{j}\right) \cdot\left(\lambda\left(v_{1}-v_{0}\right)-\left(v_{i}-v_{0}\right)\right)
$$

Using Lemma 2.1(iii) it follows that

$$
h^{\prime} \cdot\left(v^{\prime}-v_{i}\right)=\lambda\left[\gamma_{1} h_{1} \cdot\left(v_{1}-v_{0}\right)-\gamma_{i} h_{i} \cdot\left(v_{i}-v_{0}\right)\right] .
$$

Again from Lemma 2.1(iii) we have that $h_{1} \cdot\left(v_{i}-v_{0}\right)=0$ and $h_{i} \cdot\left(v_{1}-v_{0}\right)=0$. This implies $h_{1} \cdot v_{0}=h_{1} \cdot v_{i}$ and $h_{i} \cdot v_{0}=h_{i} \cdot v_{1}$. Therefore

$$
h^{\prime} \cdot\left(v^{\prime}-v_{i}\right)=\lambda\left(\gamma_{1} h_{1}+\gamma_{i} h_{i}\right) \cdot\left(v_{1}-v_{i}\right) .
$$

Substituting the expression for $h_{0}$ we get

$$
h^{\prime} \cdot\left(v^{\prime}-v_{i}\right)=\lambda\left(\gamma_{1} h_{1}+\gamma_{i} h_{i}\right) \cdot\left(v_{1}-v_{i}\right)=\lambda\left(-h_{0}-\sum_{\substack{k=2 \\ k \neq i}}^{n} \gamma_{k} h_{k}\right) \cdot\left(v_{1}-v_{i}\right)=0,
$$

where we again apply Lemma 2.1(iii) in the last step. Hence, for all $i \in\{2, \cdots, n\}$, $h^{\prime} \cdot\left(v^{\prime}-v_{i}\right)=0$ which implies $h^{\prime} \perp \mathcal{F}_{0}^{\prime}$.

Finally, we must show $h^{\prime}$ points out of $\mathcal{S}_{1}$. Suppose instead it points out of $\mathcal{S}^{\prime}$. Then by Lemma 2.1(vi), $h^{\prime} \cdot\left(v_{i}-v_{0}\right)>0$, for $i=1, \ldots, n$. Using (6.2) and Lemma 2.1(ii), we have $h^{\prime} \cdot\left(v_{i}-v_{0}\right)=\lambda \gamma_{i} h_{i} \cdot\left(v_{i}-v_{0}\right)$, for $i=2, \ldots, n$. We know $\lambda, \gamma_{i}>0$ and by Lemma 2.1(iv), $h_{i} \cdot\left(v_{i}-v_{0}\right)<0$. Thus, $h^{\prime} \cdot\left(v_{i}-v_{0}\right)<0$, a contradiction. Therefore, it must be that $h^{\prime}$ points out of $\mathcal{S}_{1}$. 
Lemma 6.4. Suppose Assumption 6.1 holds. There exists $v^{\prime} \in\left(v_{0}, v_{1}\right)$, such that $\mathcal{B} \cap$ cone $\left(\mathcal{S}_{1}\right) \neq \mathbf{0}$. Moreover, $h^{\prime} \cdot b_{1}<0$ with $b_{1}$ provided by Lemma 6.2.

Proof. Observe that

$$
\operatorname{cone}\left(\mathcal{S}_{1}\right)=\left\{y \in \mathbb{R}^{n} \mid h^{\prime} \cdot y \leq 0, h_{j} \cdot x \leq 0, \quad j \in\{2, \cdots, n\}\right\}
$$

We show there is an interval of values for $\lambda$ such that $0 \neq b_{1} \in \mathcal{B} \cap \operatorname{cone}\left(\mathcal{S}_{1}\right)$, where $b_{1}$ is provided by Lemma 6.2. First, since $b_{1} \in \mathcal{B} \cap \mathcal{C}_{1}$ we know $h_{j} \cdot b_{1} \leq 0$ for $j \in\{2, \cdots, n\}$. We must only show that there exists $\lambda \in(0,1)$ such that $h^{\prime} \cdot b_{1} \leq 0$. From Lemma 6.3 we have

$$
h^{\prime} \cdot b_{1}=\gamma_{1} h_{1} \cdot b_{1}+\lambda\left(\sum_{j=2}^{n} \gamma_{j} h_{j} \cdot b_{1}\right) .
$$

We claim that $c:=\left(\sum_{j=2}^{n} \gamma_{j} h_{j} \cdot b_{1}\right) \neq 0$. It is known that $\gamma_{j} h_{j} \cdot b_{1} \leq 0$ for $j \in\{2, \cdots, n\}$ since $b_{1} \in \mathcal{B} \cap \mathcal{C}_{1}$ and $\gamma_{j}>0$. Suppose by way of contradiction that $c=0$. This implies $h_{j} \cdot b_{1}=0$ for $j \in\{2, \cdots, n\}$. This implies $b_{1}$ takes the form $b_{1}=\xi\left(v_{1}-v_{0}\right)$ where $\xi \in \mathbb{R}$. But $h_{0} \cdot\left(v_{1}-v_{0}\right)>0$ by Lemma $2.1(\mathrm{vi})$, so $\xi>0$. Since $v_{1}-v_{0} \in \operatorname{cone}(\mathcal{S})$, we deduce $0 \neq b_{1} \in \mathcal{B} \cap \operatorname{cone}(\mathcal{S})$, a contradiction. Hence $c \neq 0$.

Since $c \neq 0$, we can solve the linear equation (6.3) in $\lambda$ when $h^{\prime} \cdot b_{1}=0$. This yields

$$
\lambda^{*}:=\frac{-\gamma_{1} h_{1} \cdot b_{1}}{\left(\sum_{j=2}^{n} \gamma_{j} h_{j} \cdot b_{1}\right)} .
$$

By the linearity of (6.3), we have $h^{\prime} \cdot b_{1}<0$ for each $\lambda \in\left(\lambda^{*}, 1\right)$. If we set $v *=\lambda^{*} v_{1}+$ $\left(1-\lambda^{*}\right) v_{0}$ then for every $v^{\prime} \in\left(v^{*}, v_{1}\right)$ we have $h^{\prime} \cdot b_{1}<0$, implying $b_{1} \in \mathcal{B} \cap \operatorname{cone}\left(\mathcal{S}_{1}\right)$.

Lemma 6.5. Suppose Assumption 6.1 holds. If the invariance conditions for $\mathcal{S}$ are solvable, then $\mathcal{S}_{1} \stackrel{\mathcal{S}_{1}}{\longrightarrow} \mathcal{F}_{0}$ by affine feedback.

Proof. By Lemma 6.4, we have $\mathcal{B} \cap \operatorname{cone}\left(\mathcal{S}_{1}\right) \neq 0$. We show that the invariance conditions are solvable for $\mathcal{S}_{1}$. First, consider the vertex $v^{\prime}$. By assumption there exist control inputs $u_{0}, u_{1} \in \mathbb{R}^{m}$ such that the invariance conditions for $\mathcal{S}$ at $v_{0}$ and $v_{1}$ are satisfied, i.e.

$$
\begin{aligned}
& y_{0}:=A v_{0}+B u_{0}+a \in \operatorname{cone}(\mathcal{S}) \\
& y_{1}:=A v_{1}+B u_{1}+a \in \mathcal{B} \cap \mathcal{C}_{1} .
\end{aligned}
$$


In particular, $h_{j} \cdot y_{i} \leq 0$ for $i=0,1$ and $j=2, \ldots, n$. Now by Lemma 6.4 , there exists $\lambda \in(0,1)$ such that with $v^{\prime}:=\lambda v_{1}+(1-\lambda) v_{0}, h^{\prime} \cdot b_{1}<0$ and $h_{j} \cdot b_{1} \leq 0$ for $j=2, \ldots, n$. Let $w_{1}$ be such that $b_{1}=B w_{1}$. Set $\epsilon_{1}>0$ and let $u^{\prime}:=\lambda u_{1}+(1-\lambda) u_{0}+\epsilon_{1} w_{1}$. Then

$$
y^{\prime}:=A v^{\prime}+B u^{\prime}+a=\lambda y_{1}+(1-\lambda) y_{0}+\epsilon_{1} b_{1} .
$$

Thus, $h_{j} \cdot y^{\prime} \leq 0$ for $j=2, \ldots, n$ and for $\epsilon_{1}>0$ sufficiently large, $h^{\prime} \cdot y^{\prime}<0$. That is, the invariance conditions for $\mathcal{S}_{1}$ are solvable at $v^{\prime}$.

Next consider $v_{1}$. Since the invariance conditions for $\mathcal{S}_{1}$ at $v_{1}$ are identical to those for $\mathcal{S}$ at $v_{1}$, and since the latter are by assumption solvable, the former are also solvable. Finally, consider vertices $v_{i}, i=2, \ldots, n$. There exist control inputs $u_{i} \in \mathbb{R}^{m}$ such that

$$
y_{i}:=A v_{i}+B u_{i}+a
$$

satisfy $h_{j} \cdot y_{i} \leq 0$ for $j=2, \ldots, i-1, i+1, \ldots, n$. As above let $w_{1}$ be such that $b_{1}=B w_{1}$. Set $\epsilon_{1}>0$ and let $u_{i}^{\prime}:=u_{i}+\epsilon_{1} w_{1}$. Then the closed-loop vector field for $\mathcal{S}_{1}$ at $v_{i}$ is

$$
y_{i}^{\prime}=A v_{i}+B u_{i}^{\prime}+a=y_{i}+\epsilon_{1} b_{1} .
$$

Thus, $h_{j} \cdot y_{i}^{\prime} \leq 0$ for $j=2, \ldots, i-1, i+1, \ldots, n$ and for $\epsilon_{1}>0$ sufficiently large, $h^{\prime} \cdot y_{i}^{\prime}<0$. That is, the invariance conditions for $\mathcal{S}_{1}$ are solvable at $v_{i}$. In sum, we can apply Theorem 4.2 to obtain that $\mathcal{S}_{1} \stackrel{\mathcal{S}_{1}}{\longrightarrow} \mathcal{F}_{0}$ by affine feedback.

Lemma 6.6. Suppose Assumption 6.1 holds. If the invariance conditions for $\mathcal{S}$ are solvable then

(i) The invariance conditions for $\mathcal{S}^{\prime}$ are solvable.

(ii) $\left(-h^{\prime}\right) \cdot b_{m_{k}}>0, \quad k=2, \ldots, p$.

Proof. First we prove (i). By assumption the invariance conditions are solvable for $\mathcal{S}$, and since the invariance conditions for $\mathcal{S}^{\prime}$ are identical (the only facet which changed for $\mathcal{S}^{\prime}$ is $\mathcal{F}_{0}$, which plays no role in invariance conditions), they are also solvable for $\mathcal{S}^{\prime}$. 
Next we prove (ii). Using the expressions for $h^{\prime}$ and $h_{0}$ in Lemma 6.3, $h^{\prime}$ can be re-expressed as

$$
h^{\prime}=\gamma_{1}(1-\lambda) h_{1}-\lambda h_{0},
$$

where $\gamma_{1}>0$ and $\lambda \in(0,1)$. Since $b_{m_{k}} \in \mathcal{B} \cap \mathcal{C}_{m_{k}}$, we have $h_{1} \cdot b_{m_{k}} \leq 0$, for $k=2, \ldots, p$. Also by Lemma $6.2, h_{0} \cdot b_{m_{k}}>0$, for $k=2, \ldots, p$. Thus,

$$
\left(-h^{\prime}\right) \cdot b_{m_{k}}=-\gamma_{1}(1-\lambda) h_{1} \cdot b_{m_{k}}+\lambda h_{0} \cdot b_{m_{k}}>0, \quad k=2, \ldots, p .
$$

We have demonstrated the first step of a triangulation procedure that partitions $\mathcal{S}$ into sub-simplices on which sub-reach control problems are solvable. Now we present a triangulation algorithm that iterates on the presented subdivision method. It consists of $p$ iterations, one for each set $\left\{v_{m_{k}}, \ldots, v_{m_{k}+r_{k}-1}\right\}, k=1, \ldots, p$.

\section{Subdivision Algorithm.}

1. Set $m_{0}:=0 ; k:=1 ; v_{i}^{0}:=v_{i}, i=0, \ldots, n$.

\section{Subdivision:}

- Set $v_{i}^{k}:= \begin{cases}v_{i}^{k-1}, & i \in I \backslash\left\{m_{(k-1)}\right\} \\ v_{0}^{k-1}, & i=m_{(k-1)}\end{cases}$

- Select $v_{0}^{k} \in\left(v_{0}, v_{m_{k}}\right)$ according to Lemma 6.4.

- Set $\mathcal{S}_{k}:=\operatorname{co}\left\{v_{0}^{k}, \ldots, v_{n}^{k}\right\}$.

- If $k<p$, set $k:=k+1$ and repeat step (2).

3. Termination:

$-\operatorname{Set} v_{i}^{p+1}:= \begin{cases}v_{0}, & i=0 \\ v_{i}^{p}, & i \in I \backslash\left\{m_{p}\right\} \\ v_{0}^{p}, & i=m_{p} .\end{cases}$

- Set $\mathcal{S}_{p+1}:=\operatorname{co}\left\{v_{0}^{p+1}, \ldots, v_{n}^{p+1}\right\}$. 
Let $\mathcal{F}_{0}^{k}=$ co $\left\{v_{1}^{k}, \ldots, v_{n}^{k}\right\}$ denote the exit facet of $\mathcal{S}_{k}$. The triangulation generated by the algorithm has the property that

$$
\mathcal{S}_{k} \cap \mathcal{S}_{k-1}=\mathcal{F}_{0}^{k}, \quad k=2, \ldots, p+1,
$$

and closed-loop trajectories follow paths through sub-simplices with decreasing indices. Thus, $\mathcal{S} \stackrel{\mathcal{S}}{\longrightarrow} \mathcal{F}_{0}$ is achieved by implementing affine controllers that achieve $\mathcal{S}_{k} \stackrel{\mathcal{S}_{k}}{\longrightarrow} \mathcal{F}_{0}^{k}$ for $k=1, \ldots, p+1$. In order to guarantee that switching occurs in the proper sequence (with decreasing simplex indices), and to avoid chattering caused by measurement errors, a supervisor should accompany the implementation of the piecewise affine feedback. The supervisor has two functions:

(i) Once the piecewise affine controller has switched to simplex $\mathcal{S}_{k}$, then all affine controllers for $\mathcal{S}_{j}, j>k$, are disabled.

(ii) The affine controller for $\mathcal{S}_{j}$ is released to $\mathcal{S}_{j-1}$ only after the closed-loop trajectory exits $\mathcal{S}_{j}$. Thus, at a point $x \in \mathcal{S}_{j} \cap \mathcal{S}_{j-1}$, the controller for the simplex with the higher index is used.

Theorem 6.1. Suppose Assumption 6.1 holds. If the invariance conditions for $\mathcal{S}$ are solvable, then $\mathcal{S} \stackrel{\mathcal{S}}{\longrightarrow} \mathcal{F}_{0}$ by piecewise affine feedback.

Proof. Form the triangulation $\left\{\mathcal{S}_{1}, \ldots, \mathcal{S}_{p+1}\right\}$ of $\mathcal{S}$ based on the Subdivision Algorithm. To show that $\mathcal{S} \stackrel{\mathcal{S}}{\longrightarrow} \mathcal{F}_{0}$ by piecewise affine feedback, we first show that $\mathcal{S}_{k} \stackrel{\mathcal{S}_{k}}{\longrightarrow} \mathcal{F}_{0}^{k}$ by affine feedback for $k=1, \ldots, p+1$.

Lemmas 6.4 and 6.5 depend on two properties of $\mathcal{S}$ : condition (P6) and solvability of its invariance conditions. Let

$$
\mathcal{S}_{k}^{\prime}:=\operatorname{co}\left\{v_{0}, v_{1}^{k}, \ldots, v_{n}^{k}\right\}
$$

with $v_{i}^{k}$ as in step (2) of the algorithm. Then $\mathcal{S}_{k} \subset \mathcal{S}_{k}^{\prime}$ and $\mathcal{S}_{k}^{\prime}$ takes the role of $\mathcal{S}$ in Lemmas 6.2, 6.4, and 6.5. Thus, we must verify that $\mathcal{S}_{k}^{\prime}$ inherits the needed properties 
of $\mathcal{S}$. However, Lemma 6.6 guarantees by an inductive argument that for each successor $\mathcal{S}_{k}^{\prime}$, the invariance conditions remain solvable and $\left(-h^{k}\right) \cdot b_{m_{k}}>0$ for $k=2, \ldots, p$. The latter statement means that Lemma 6.2 applies to each $\mathcal{S}_{k}$ (with $h^{k}$ representing the $k$ th iterate of $h^{\prime}$ ); and this, in turn, means Lemmas 6.4 and 6.5 also apply to $\mathcal{S}^{k} \subset \mathcal{S}_{k}^{\prime}$. We conclude $\mathcal{S}_{k} \stackrel{\mathcal{S}_{k}}{\longrightarrow} \mathcal{F}_{0}^{k}$ by affine feedback for $k=1, \ldots, p$.

Next consider $\mathcal{S}_{p+1}$. By Lemma 6.6, the invariance conditions for $\mathcal{S}_{p+1}$ are solvable $\left(\mathcal{S}_{p+1}\right.$ and $\mathcal{S}$ share the same invariance conditions since they only differ in their exit facets). Now let $\mathcal{G}_{p+1}:=\mathcal{S}_{p+1} \cap \mathcal{O}$. Then by the algorithm,

$$
\mathcal{G}_{p+1}=\text { co }\left\{v_{2}, \ldots, v_{m_{1}-1}, v_{m_{1}+1}, \ldots, v_{m_{p}-1}, v_{m_{p}+1}, \ldots, v_{m+p}\right\}
$$

We can see that the algorithm has removed the $p$ vertices $v_{1}, v_{m_{2}}, \ldots, v_{m_{p}}$ and this has the effect to break up the dependencies of $\mathcal{B}$ associated with $\mathcal{G}$. There remain $m$ vectors in $\mathcal{B}$ associated with $\mathcal{G}_{p+1}$ such that

$$
\mathcal{B}=\operatorname{span}\left\{b_{2}, \ldots, b_{m_{1}-1}, b_{m_{1}+1}, \ldots, b_{m_{p}-1}, b_{m_{p}+1}, \ldots, b_{m+p}\right\}
$$

is a maximal linearly independent set. Therefore, we can apply Theorem 4.3 to obtain $\mathcal{S}_{p+1} \stackrel{\mathcal{S}_{p+1}}{\longrightarrow} \mathcal{F}_{0}^{p+1}$.

Finally, we must prove that trajectories progress through sub-simplices with decreasing indices (thereby guaranteeing that the supervisor cannot block). Consider w.l.og. the boundary between $\mathcal{S}_{1}$ and $\mathcal{S}_{2}$ given by $\mathcal{F}_{0}^{1}=$ co $\left\{v^{\prime}, v_{2}, \ldots, v_{n}\right\}$, and let $u=K_{1} x+g_{1}$ be the affine feedback obtained for $\mathcal{S}_{1}$. We must show that for any $x_{0} \in \mathcal{S}_{1} \backslash \mathcal{F}_{0}^{1}$, closed-loop trajectories do not reach $\mathcal{F}_{0}^{1}$. This can be deduced from the proof of Lemma 6.5 where it is shown that the controls $\left\{u^{\prime}, u_{2}, \ldots, u_{n}\right\}$ can be selected so that

$$
\begin{aligned}
& h^{\prime} \cdot\left(A v^{\prime}+B u^{\prime}+a\right)<0 \\
& h^{\prime} \cdot\left(A v_{i}+B u_{i}+a\right)<0, \quad i=2, \ldots, n .
\end{aligned}
$$

By convexity, $h^{\prime} \cdot\left(A x+B\left(K_{1} x+g_{1}\right)+a\right)<0$ for all $x \in \mathcal{F}_{0}^{1}$, from which the result easily follows. 


\subsection{Main Result}

Theorem 6.2. Suppose Assumption 4.1 holds. Then the following statements are equivalent:

(1) $\mathcal{S} \stackrel{\mathcal{S}}{\longrightarrow} \mathcal{F}_{0}$ by piecewise affine feedback.

(2) $\mathcal{S} \stackrel{\mathcal{S}}{\longrightarrow} \mathcal{F}_{0}$ by open-loop controls.

Proof. (1) $\Longrightarrow(2)$ is obvious.

$(2) \Longrightarrow(1)$ Suppose $\mathcal{S} \stackrel{\mathcal{S}}{\longrightarrow} \mathcal{F}_{0}$ by open-loop controls. By Theorem 3.1, the invariance conditions are solvable. Let $\mathcal{G}:=\mathcal{S} \cap \mathcal{O}$. If $\mathcal{G}=\emptyset$, then by Theorem $4.1, \mathcal{S} \stackrel{\mathcal{S}}{\longrightarrow} \mathcal{F}_{0}$ by affine feedback. Suppose instead $\mathcal{G} \neq \emptyset$. If $\mathcal{B} \cap \operatorname{cone}(\mathcal{S}) \neq \mathbf{0}$, then by Theorem 4.2 , $\mathcal{S} \stackrel{\mathcal{S}}{\longrightarrow} \mathcal{F}_{0}$ by affine feedback. Suppose instead $\mathcal{B} \cap \operatorname{cone}(\mathcal{S})=\mathbf{0}$. In this case, by reordering indices, $\mathcal{G}=\operatorname{co}\left\{v_{1}, \ldots, v_{\kappa+1}\right\}$, where $0 \leq \kappa<n$. By Theorem $3.2, \mathcal{B} \cap \mathcal{C}_{i} \neq \mathbf{0}$ for $i \in I_{\mathcal{G}}$. Define $\hat{\mathcal{B}}=\operatorname{span}\left\{b_{1}, \ldots, b_{\kappa+1} \mid b_{i} \in \mathcal{B} \cap \mathcal{C}_{i}\right\}$. Notice that by Lemma 5.2 , the invariance conditions remain solvable using $\hat{\mathcal{B}}$. Also, by Lemma $5.3, \mathcal{G}$ is unchanged. Finally, since $\mathcal{B} \cap \mathcal{C}_{i} \subset \hat{\mathcal{B}} \cap \mathcal{C}_{i}$, we have $\hat{\mathcal{B}} \cap \mathcal{C}_{i} \neq \mathbf{0}$, for $i \in I_{\mathcal{G}}$. Thus, we can perform the affine feedback transformation given in (5.3)-(5.5) and redefine $m$ and $\mathcal{B}$ such that $\mathcal{B}=\operatorname{span}\left\{b_{1}, \ldots, b_{m}\right\}$ for some $b_{i} \in \mathcal{B} \cap \mathcal{C}_{i}$. If $\kappa<m$, then by Theorem $4.3, \mathcal{S} \stackrel{\mathcal{S}}{\longrightarrow} \mathcal{F}_{0}$ by affine feedback. Suppose instead $\kappa \geq m$. In this case the reach control indices can be defined, and by Lemma $6.1, \mathcal{B}_{k} \not \subset \mathcal{F}_{0}$ for $k=1, \ldots, p$. Thus, all conditions of Assumption 6.1 hold. By Theorem $6.1, \mathcal{S} \stackrel{\mathcal{S}}{\longrightarrow} \mathcal{F}_{0}$ by piecewise affine feedback. 


\section{Chapter 7}

\section{Examples}

In this chapter, various examples are presented which illustrate the Reach Control Problem and its solution using the appropriate control assignment according to the theory developed in the previous chapters. Examples in which RCP is solvable using affine feedback are first presented. We then focus on examples where continuous state feedback cannot solve the problem (due to existence of closed-loop equilibria within the simplex). In these cases, we apply the subdivision algorithm to obtain a piecewise affine feedback that solves RCP. The geometric sufficient conditions for solvability of RCP, which focus on the relationship between $\mathcal{B}$, $\operatorname{cone}(\mathcal{S})$ and $\mathcal{G}$ are verified for each example.

\subsection{RCP solution using affine feedback : $\mathcal{G}=\emptyset$.}

Simple examples in which $\mathcal{G}=\emptyset$ and the invariance conditions are solvable are first presented. By, Theorem 4.1 we have $\mathcal{S} \stackrel{\mathcal{S}}{\longrightarrow} \mathcal{F}_{0}$ by affine feedback. 


\subsubsection{Example 1}

Consider the simplex $\mathcal{S}$ determined by $v_{0}=(0,0), v_{1}=(0.5,1)$ and $v_{2}=(1,1)$. Let the affine dynamics on $\mathcal{S}$ be defined as

$$
\dot{x}=\left[\begin{array}{rr}
-1 & 1 \\
0 & 0
\end{array}\right] x+\left[\begin{array}{l}
0 \\
1
\end{array}\right] u+\left[\begin{array}{l}
1 \\
1
\end{array}\right] .
$$

The set of points $\mathcal{O}$ where $A x+a \in \mathcal{B}$ is given by

$$
\mathcal{O}=\left\{x \mid x_{2}-x_{1}+1=0\right\}
$$

Hence $\mathcal{S} \cap \mathcal{O}=\mathcal{G}=\emptyset$. The normal vectors of the simplex $\mathcal{S}$ are $h_{0}=(0,1), h_{1}=(1,-1)$ and $h_{2}=(-1,0.5)$. This results in the following invariance conditions for $\mathcal{S}$.

At $v_{0}$

$$
\begin{aligned}
& h_{1} \cdot y_{0} \leq 0 \Longrightarrow(1,-1) \cdot\left(1,1+u_{0}\right) \leq 0 \\
& h_{2} \cdot y_{0} \leq 0 \Longrightarrow(-1,0.5) \cdot\left(1,1+u_{0}\right) \leq 0
\end{aligned}
$$

At $v_{1}$

$$
h_{2} \cdot y_{1} \leq 0 \Longrightarrow(-1,0.5) \cdot\left(1.5,1+u_{1}\right) \leq 0
$$

At $v_{2}$

$$
h_{1} \cdot y_{2} \leq 0 \Longrightarrow(1,-1) \cdot\left(1,1+u_{2}\right) \leq 0
$$

In order to satisfy the invariance conditions the control inputs at the vertices of $\mathcal{S}$ can be chosen as $u_{0}=0.5, u_{1}=-2$ and $u_{2}=5$. The affine feedback law can be solved using the following equation.

$$
\begin{aligned}
& u_{i}=F v_{i}+g \quad i=0, \ldots, 2 \\
& {\left[\begin{array}{c}
-v_{0}^{T}-1 \\
-v_{1}^{T}-1 \\
-v_{2}^{T}-1
\end{array}\right]\left[\begin{array}{c}
F^{T} \\
g^{T}
\end{array}\right]=\left[\begin{array}{l}
u_{0} \\
u_{1} \\
u_{2}
\end{array}\right] .}
\end{aligned}
$$


Solution of the above yields an affine feedback law:

$$
u=\left[\begin{array}{ll}
14 & -9.5
\end{array}\right] x+0.5 \quad x \in \mathcal{S}
$$

Simulation of the closed-loop system is shown in Figure 7.1. The vector field satisfies the invariance conditions and points out of $\mathcal{S}$ only though the exit facet $\mathcal{F}_{0}$. All trajectories originating within $\mathcal{S}$ will leave via $\mathcal{F}_{0}$. Therefore we have $\mathcal{S} \stackrel{\mathcal{S}}{\longrightarrow} \mathcal{F}_{0}$ by affine feedback.

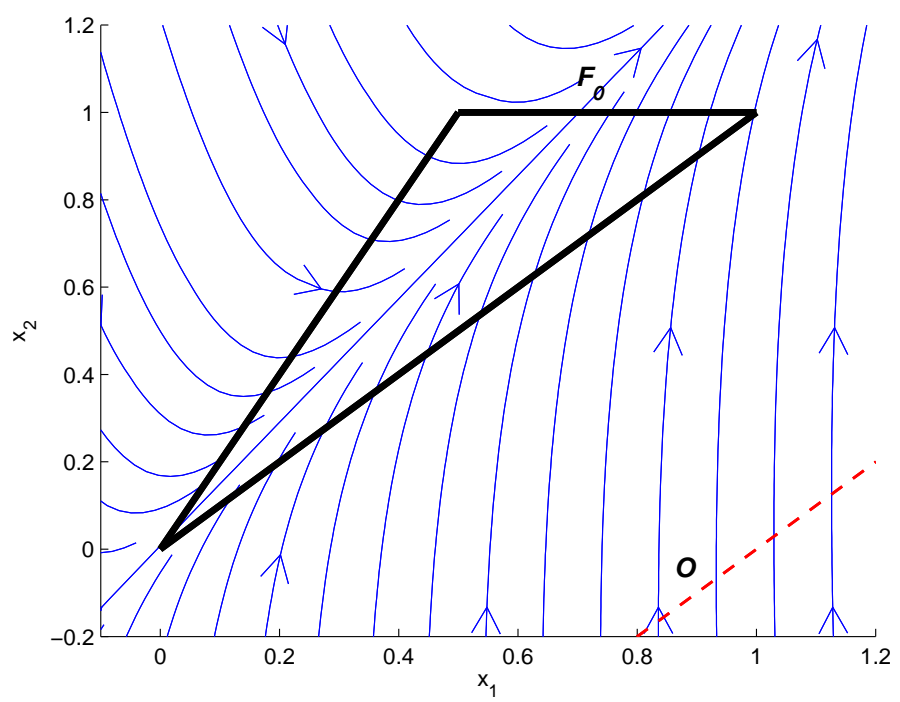

Figure 7.1: Example 1: Closed loop vector field on $\mathcal{S}$

\subsubsection{Example 2}

Consider another simplex $\mathcal{S}$ determined by $v_{0}=(0,1), v_{1}=(1,0)$ and $v_{2}=(-1,0)$, and also consider the affine dynamics:

$$
\dot{x}=\left[\begin{array}{ll}
0 & 1 \\
0 & 0
\end{array}\right] x+\left[\begin{array}{l}
0 \\
1
\end{array}\right] u+\left[\begin{array}{l}
1 \\
1
\end{array}\right] \text {. }
$$

We have

$$
\mathcal{O}=\left\{x \mid x_{2}=-1\right\}
$$


Thus $\mathcal{S} \cap \mathcal{O}=\mathcal{G}=\emptyset$. The normal vectors of the simplex $\mathcal{S}$ are $h_{0}=(0,-1), h_{1}=(-1,1)$ and $h_{2}=(1,1)$. The corresponding invariance conditions for $\mathcal{S}$ are:

At $v_{0}$

$$
\begin{aligned}
& h_{1} \cdot y_{0} \leq 0 \Longrightarrow \quad(-1,1) \cdot\left(\left(2,1+u_{0}\right) \leq 0\right. \\
& h_{2} \cdot y_{0} \leq 0 \Longrightarrow \quad(1,1) \cdot\left(2,1+u_{0}\right) \leq 0
\end{aligned}
$$

At $v_{1}$

$$
h_{2} \cdot y_{1} \leq 0 \Longrightarrow(1,1) \cdot\left(1,1+u_{1}\right) \leq 0
$$

At $v_{2}$

$$
h_{1} \cdot y_{2} \leq 0 \Longrightarrow(-1,1) \cdot\left(1,1+u_{2}\right) \leq 0
$$

In order to satisfy the invariance conditions we choose the control inputs at the vertices as $u_{0}=-4, u_{1}=-4$ and $u_{2}=0$. The choice of control inputs at the vertices yields an affine feedback law

$$
u=\left[\begin{array}{ll}
-2 & -2
\end{array}\right] x-2 \quad x \in \mathcal{S}
$$

Simulation of the closed-loop system is shown in Figure 7.2. The vector field satisfies the invariance conditions and points out of $\mathcal{S}$ only though the exit facet $\mathcal{F}_{0}$. Note the equilibrium point located on the set $\mathcal{O}$ which is outside of $\mathcal{S}$. All trajectories originating within $\mathcal{S}$ will leave via $\mathcal{F}_{0}$, and RCP is therefore solved using affine feedback.

\subsection{RCP solution using affine feedback: $\mathcal{G} \neq \emptyset$ and $\mathcal{B} \cap \operatorname{cone}(\mathcal{S}) \neq 0$}

In the previous examples solvability of the invariance conditions was sufficient to solve $\mathrm{RCP}$ by affine feedback. We consider the case where $\mathcal{G} \neq \emptyset$ and Assumption 4.1 holds. If $\mathcal{B} \cap \operatorname{cone}(\mathcal{S}) \neq \mathbf{0}$, by Theorem 4.2 we have $\mathcal{S} \stackrel{\mathcal{S}}{\longrightarrow} \mathcal{F}_{0}$ by affine feedback provided the invariance conditions are solvable. 


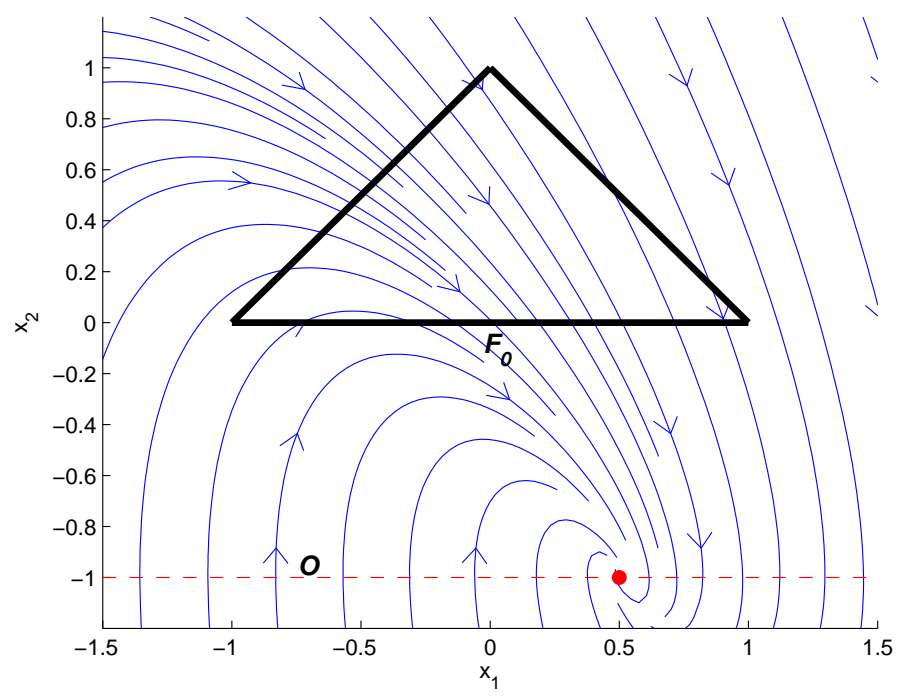

Figure 7.2: Example 2: Closed loop vector field on $\mathcal{S}$.

\subsubsection{Example 3}

Let the simplex $\mathcal{S}$ be determined by the vertices $v_{0}=(0,1), v_{1}=(1,0)$ and $v_{2}=(-1,0)$.

The affine system on $\mathcal{S}$ is as follows

$$
\dot{x}=\left[\begin{array}{ll}
0 & 1 \\
0 & 0
\end{array}\right] x+\left[\begin{array}{l}
0 \\
1
\end{array}\right] u+\left[\begin{array}{l}
0 \\
0
\end{array}\right] .
$$

We have

$$
\mathcal{O}=\left\{x \mid x_{2}=0\right\}
$$

Hence $\mathcal{S} \cap \mathcal{O}=\mathcal{G}=$ co $\left\{v_{1}, v_{2}\right\}=\mathcal{F}_{0}$. The normal vectors are $h_{1}=(-1,1)$ and $h_{2}=(1,1)$.

The invariance conditions are:

At $v_{0}$

$$
\begin{aligned}
& h_{1} \cdot y_{0} \leq 0 \Longrightarrow(-1,1) \cdot\left(1, u_{0}\right) \leq 0 \\
& h_{2} \cdot y_{0} \leq 0 \Longrightarrow(1,1) \cdot\left(1, u_{0}\right) \leq 0
\end{aligned}
$$

At $v_{1}$

$$
h_{2} \cdot y_{1} \leq 0 \Longrightarrow(1,1) \cdot\left(0, u_{1}\right) \leq 0
$$


At $v_{2}$

$$
h_{1} \cdot y_{2} \leq 0 \Longrightarrow(-1,1) \cdot\left(0, u_{2}\right) \leq 0
$$

In order to satisfy the invariance conditions we choose the control inputs at the vertices of $\mathcal{S}$ as $u_{0}=-4, u_{1}=-1$ and $u_{2}=-1$. The control input choices yield an affine feedback law

$$
u=\left[\begin{array}{ll}
0 & -3
\end{array}\right] x-1 \quad x \in \mathcal{S}
$$

Simulation of the closed-loop system is given in Figure 7.3. The closed loop vector field satisfies the invariance conditions and points out of $\mathcal{S}$ only though the exit facet $\mathcal{F}_{0}$. Although $\mathcal{G}=\mathcal{F}_{0}$, the condition $\mathcal{B} \cap \operatorname{cone}(\mathcal{S}) \neq \mathbf{0}$ ensures that the closed loop vectors at the vertices of $\mathcal{G}$ both point outward of $\mathcal{F}_{0}$ in the direction of $\mathcal{B}$ guaranteeing no equilibrium. All trajectories originating within $\mathcal{S}$ will leave via $\mathcal{F}_{0}$ and RCP is solved using the affine feedback.

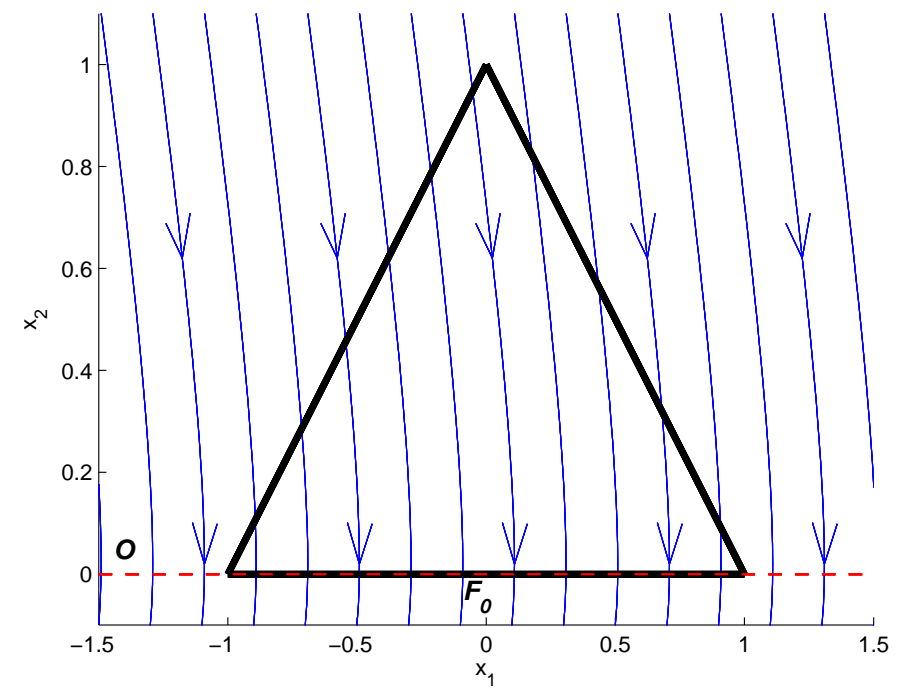

Figure 7.3: Example 3: Closed loop vector field on $\mathcal{S}$ 


\subsection{RCP solution using piecewise affine feedback}

We consider RCP where $\mathcal{G} \neq \emptyset$ and Assumption 4.1 holds. Furthermore we assume that $\mathcal{B} \cap \operatorname{cone}(\mathcal{S})=\mathbf{0}$ and there does not exist a linearly independent set $\left\{b_{1}, \ldots, b_{\kappa+1} \mid b_{i} \in \mathcal{B} \cap\right.$ $\mathcal{G}\}$. By Theorem 5.3 RCP is not solvable by continuous state feedback due to equilibrium formation on $\mathcal{S}$. If however the additional conditions (P4) - (P6) of Assumption 6.1 hold and the invariance conditions for $\mathcal{S}$ are solvable then $\mathcal{S} \stackrel{\mathcal{S}}{\longrightarrow} \mathcal{F}_{0}$ by a piecewise affine feedback according to Theorem 6.1. Examples of these cases are presented, illustrating the execution of the subdivision algorithm which yields the piecewise affine feedback to solve RCP where continuous state feedback cannot.

\subsubsection{Example 4}

Consider a simplex $\mathcal{S}$ in $\mathbb{R}^{2}$ determined by $v_{0}=(-1,1), v_{1}=(1,0)$ and $v_{2}=(0,0)$. The affine dynamics are

$$
\dot{x}=\left[\begin{array}{ll}
0 & 1 \\
0 & 0
\end{array}\right] x+\left[\begin{array}{l}
0 \\
1
\end{array}\right] u+\left[\begin{array}{l}
0 \\
0
\end{array}\right] \text {. }
$$

We have

$$
\mathcal{O}=\left\{x \mid x_{2}=0\right\}
$$

Hence $\mathcal{S} \cap \mathcal{O}=\mathcal{G}=$ co $\left\{v_{1}, v_{2}\right\}=\mathcal{F}_{0}$, with $\kappa=1$ and $m=1$. Also it can be easily verified that $\mathcal{B} \cap \operatorname{cone}(\mathcal{S})=\mathbf{0}$. By Theorem 5.3 the problem is not solvable using continuous state feedback due to the existence of equilibria on $\mathcal{S}$. This can be illustrated clearly as follows. Suppose we choose the control inputs at the vertices of $\mathcal{S}$ to satisfy the invariance conditions as $u_{0}=-\frac{3}{4}, u_{1}=-1$ and $u_{2}=1$. The resulting affine feedback law is

$$
u=\left[\begin{array}{ll}
-2 & -3.75
\end{array}\right] x+1 \quad x \in \mathcal{S}
$$

A simulation of the closed-loop system is shown in Figure 7.4. The vector field satisfies the invariance conditions however there exists an equilibrium point on the set 
$\mathcal{G}=\operatorname{co}\left\{v_{1}, v_{2}\right\}$ rendering RCP unsolved using the affine feedback. We show instead, that the problem is solvable using a piecewise affine feedback.

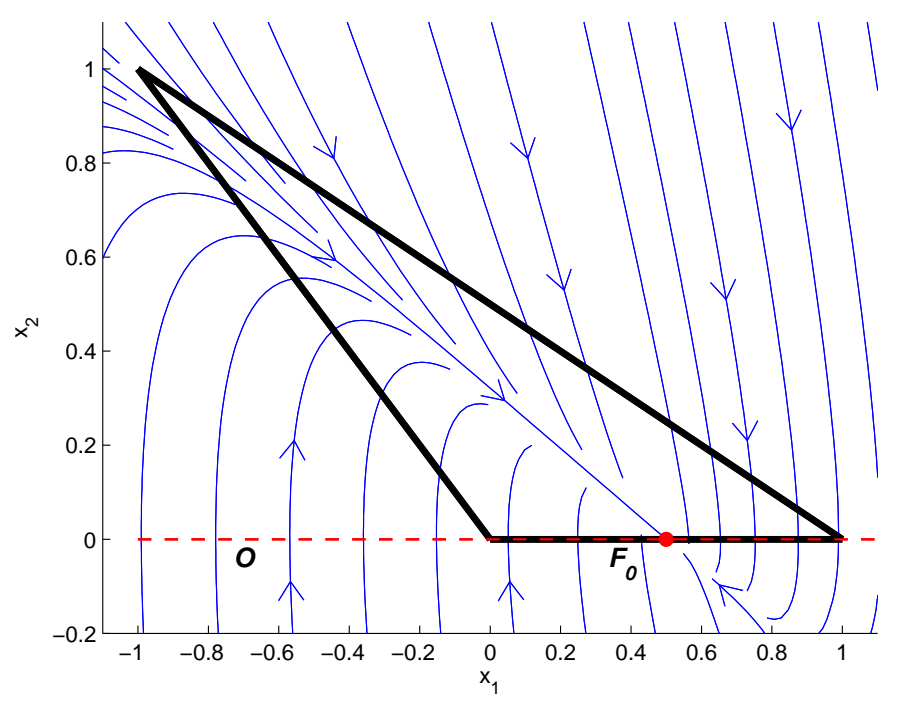

Figure 7.4: Example 4: Closed loop vector field on $\mathcal{S}$

We first verify that the relevant conditions of Assumption 6.1 which are necessary to allow for solution by piecewise affine feedback hold. By inspection $b_{1}:=(0,-1) \in$ $\mathcal{B} \cap \mathcal{C}_{1}$ and $\mathcal{B}=\operatorname{span}\left\{b_{1}\right\} . \mathcal{B}$ exhibits a single dependent cycle with respect to $\mathcal{G}$ with $b_{2}:=-b_{1} \in \mathcal{B} \cap \mathcal{C}_{2}$. It therefore clear that conditions (P3)-(P5) of Assumption 6.1 hold and furthermore it easily verified that (P6) also holds. Using Algorithm 6.2 we choose $v^{\prime}=(0.5,0.25) \in \overline{v_{0} v_{1}}$ such that we obtain the condition $\mathcal{B} \cap \operatorname{cone}\left(\mathcal{S}_{1}\right) \neq 0$ where $\mathcal{S}_{1}:=\operatorname{co}\left\{v^{\prime}, v_{1}, v_{2}\right\}$ and $\mathcal{S}_{2}:=\operatorname{co}\left\{v_{0}, v^{\prime}, v_{2}\right\}$. Also $\mathcal{F}_{0}=\operatorname{co}\left\{v^{\prime}, v_{2}\right\}$ and $h^{\prime}=(-0.25,0.5)$. In order to satisfy the invariance conditions for $\mathcal{S}_{2}$ the control inputs at the vertices are chosen to be $u_{0}=-\frac{3}{4}, u^{\prime}=-1$ and $u_{22}=1$. In order to satisfy the invariance conditions for $\mathcal{S}_{1}$ we choose $u^{\prime}=-1, u_{11}=-1$ and $u_{12}=-1$. The resulting piecewise affine feedback law for $\mathcal{S}$ is

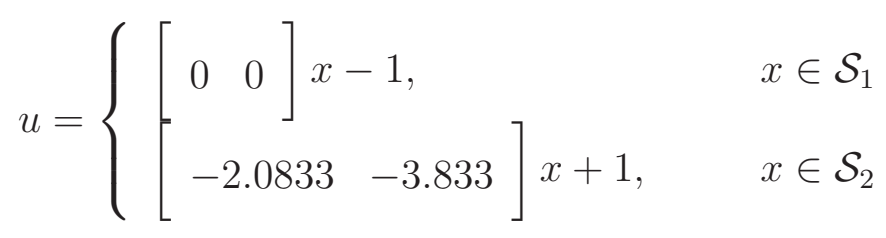


As shown below in Figure 7.5 and Figure 7.6 each sub-simplex under its individual controller has no equilibrium. The closed loop vector field for $\mathcal{S}$ using the piecewise affine feedback law is given in Figure 7.7. It is clear that now all trajectories originating in $\mathcal{S}$ will exit via $\mathcal{F}_{0}$ thus rendering RCP solved.

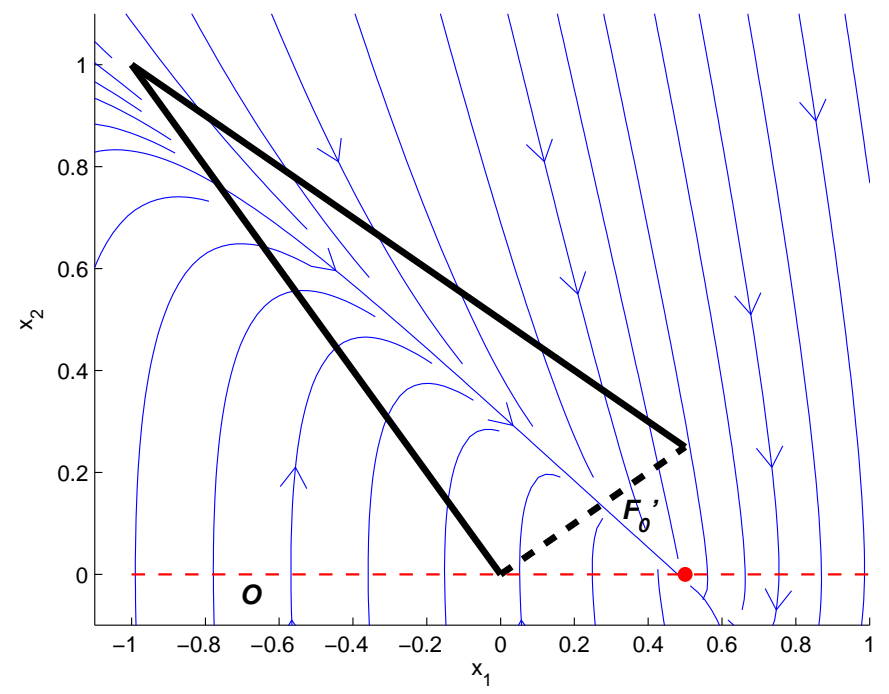

Figure 7.5: Example 4: Closed loop vector field on $\mathcal{S}_{2}$

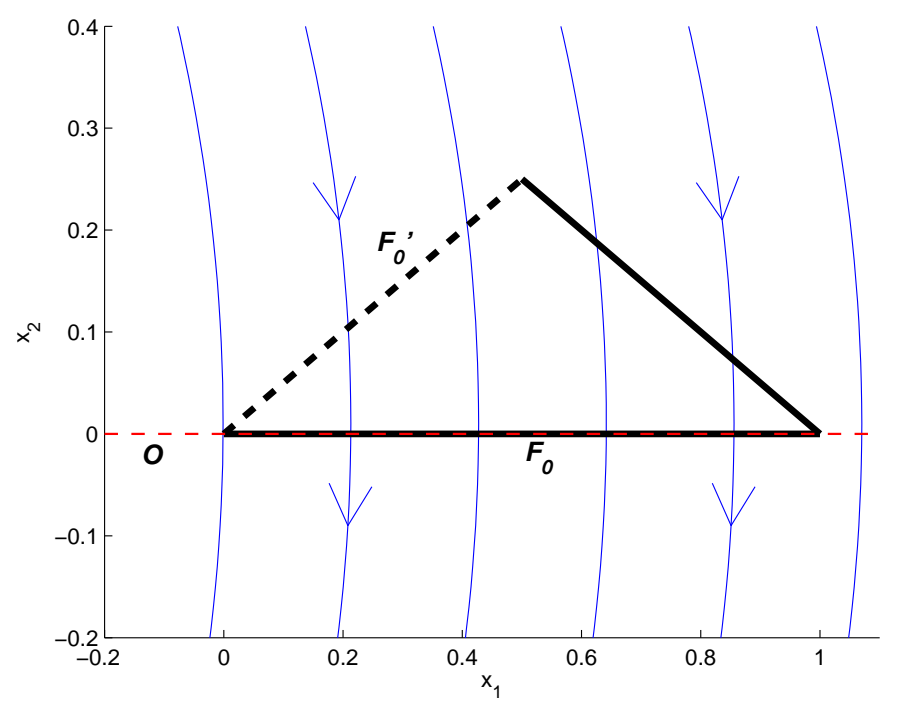

Figure 7.6: Example 4: Closed loop vector field on $\mathcal{S}_{1}$ 


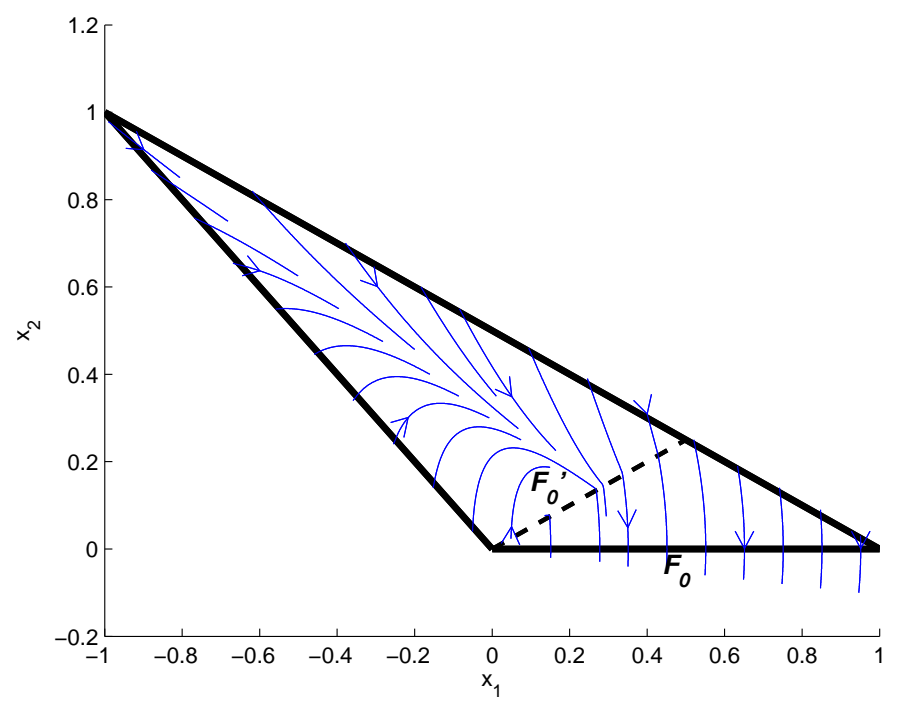

Figure 7.7: Example 4: Closed loop vector field on $\mathcal{S}$ under piecewise affine feedback

\subsubsection{Example 5}

We illustrate another case in $\mathbb{R}^{2}$. Let $\mathcal{S}$ be determined by $v_{0}=(0,0), v_{1}=(0,1)$ and $v_{2}=(1,0)$, and consider the affine dynamics

$$
\dot{x}=\left[\begin{array}{rr}
-3 & 1 \\
0 & -2
\end{array}\right] x+\left[\begin{array}{r}
-2 \\
1
\end{array}\right] u+\left[\begin{array}{l}
1 \\
1
\end{array}\right] \text {. }
$$

We have

$$
\mathcal{O}=\left\{x \mid x_{1}+x_{2}-1=0\right\}
$$

Hence $\mathcal{S} \cap \mathcal{O}=\mathcal{G}=\operatorname{co}\left\{v_{1}, v_{2}\right\}=\mathcal{F}_{0}$, with $\kappa=1$ and $m=1$. It is easily shown also that $\mathcal{B} \cap \operatorname{cone}(\mathcal{S})=\mathbf{0}$. By Theorem 5.3, RCP is therefore not solvable using a continuous state feedback. Consider however, the solution using a piecewise affine feedback.

We have $b_{1}:=(2,-1) \in \mathcal{B} \cap \mathcal{C}_{1}, \mathcal{B}=\operatorname{span}\left\{b_{1}\right\}$ and therefore a single dependent cycle of $\mathcal{B}$ with respect to $\mathcal{G}$, where $b_{2}:=-b_{1} \in \mathcal{B} \cap \mathcal{C}_{2}$. It is therefore clear that conditions (P3)-(P5) of Assumption 6.1 hold while it is also easily verified that (P6) holds. Using Algorithm 6.2 we choose $v^{\prime}=(0,0.75) \in \overline{v_{0} v_{1}}$ such that we obtain the condition $\mathcal{B} \cap \operatorname{cone}\left(\mathcal{S}_{1}\right) \neq 0$ where $\mathcal{S}_{1}:=\operatorname{co}\left\{v^{\prime}, v_{1}, v_{2}\right\}, \mathcal{S}_{2}:=\operatorname{co}\left\{v_{0}, v^{\prime}, v_{2}\right\}, \mathcal{F}_{0}=\operatorname{co}\left\{v^{\prime}, v_{2}\right\}$ and 
$h^{\prime}=(-0.75,-1)$. In order to satisfy the invariance conditions for $\mathcal{S}_{2}$ the control inputs at the vertices are chosen to be $u_{0}=u^{\prime}=u_{22}=0$. In order to satisfy the invariance conditions for $\mathcal{S}_{1}$ the control inputs at the vertices are chosen to be $u^{\prime}=0, u_{11}=0$ and $u_{12}=-2$. This choice of control inputs yields the piecewise affine feedback law

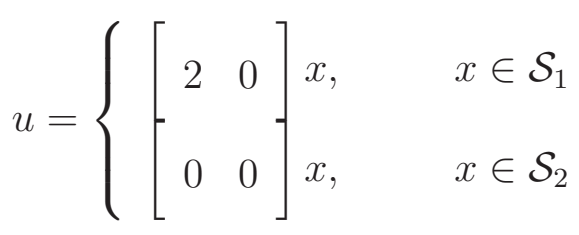

As shown below in Figure 7.8 and Figure 7.9, each sub-simplex under its individual controller has no equilibrium. The use of the piecewise feedback law solves RCP as all trajectories originating in $\mathcal{S}$ exit via $\mathcal{F}_{0}$ as shown in Figure 7.10.

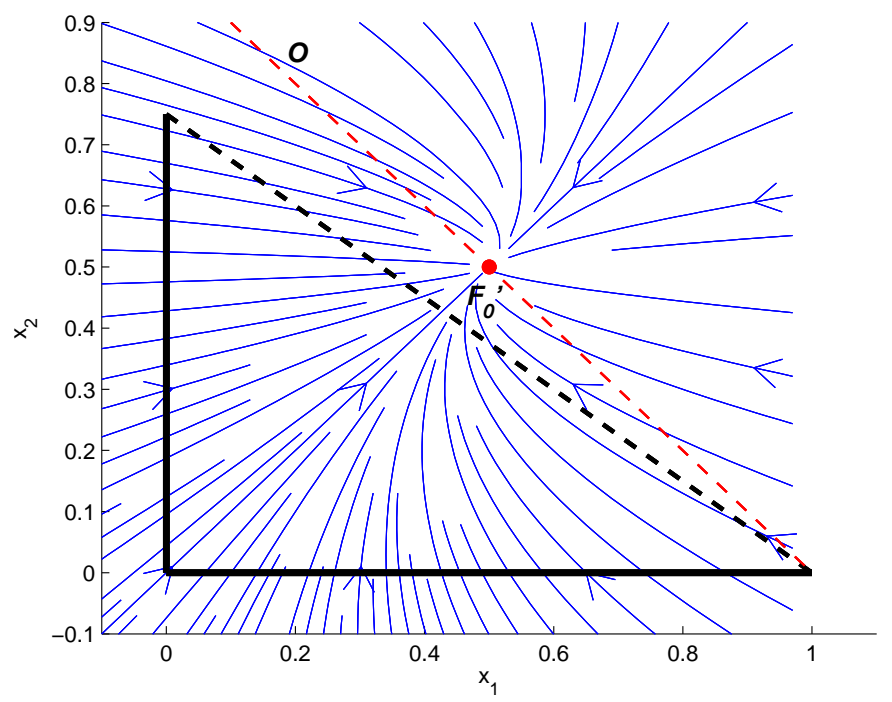

Figure 7.8: Example 5: Closed loop vector field on $\mathcal{S}_{2}$ 


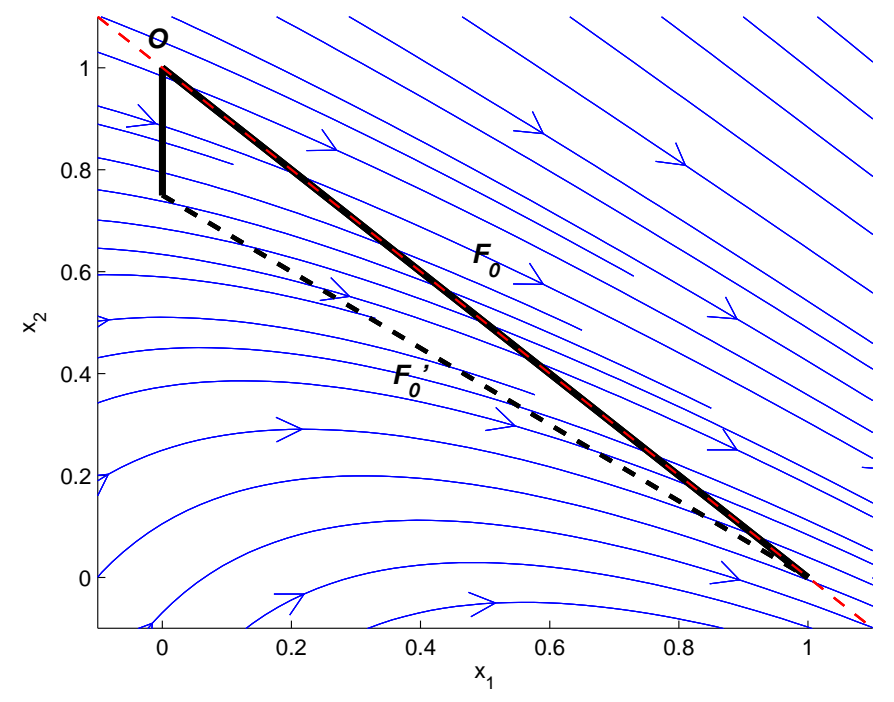

Figure 7.9: Example 5: Closed loop vector field on $\mathcal{S}_{1}$

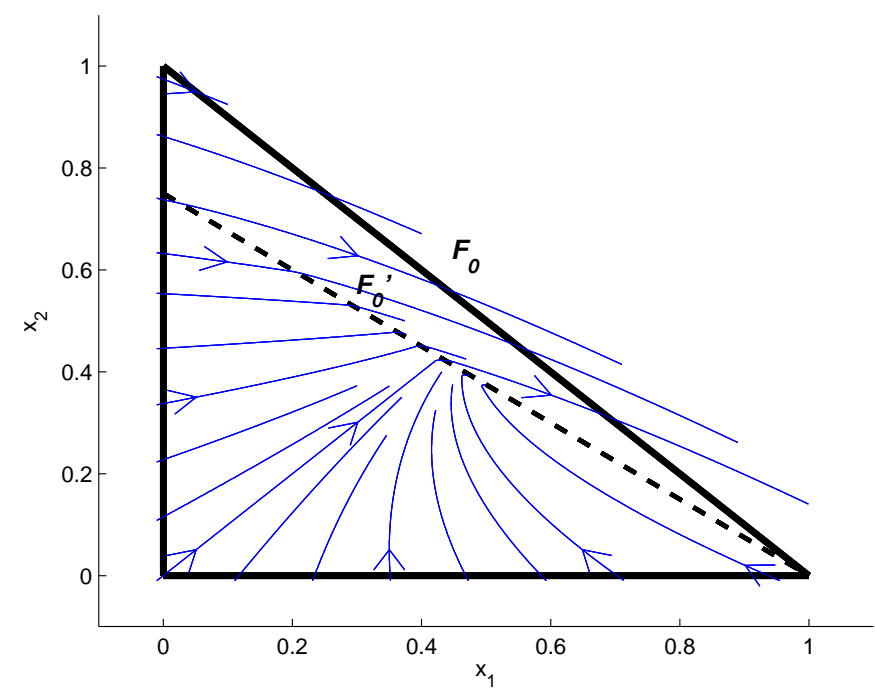

Figure 7.10: Example 5: Closed loop vector field on $\mathcal{S}$ under piecewise affine feedback

\subsubsection{Example 6}

Now we demonstrate a case in $\mathbb{R}^{3}$ with simplex $\mathcal{S}$ determined by $v_{0}=(0,1,0)$, $v_{1}=$ $(1,0,0), v_{2}=(-1,0,0)$ and $v_{3}=(0,0,1)$. The affine dynamics on $\mathcal{S}$ in $\mathbb{R}^{3}$ are defined as

$$
\dot{x}=\left[\begin{array}{rrr}
2 & 0 & 0 \\
1 & -1 & 0 \\
0 & 0 & -1
\end{array}\right] x+\left[\begin{array}{l}
2 \\
1 \\
0
\end{array}\right] u+\left[\begin{array}{l}
0 \\
0 \\
0
\end{array}\right] \text {. }
$$


With

$$
\mathcal{O}=\left\{x \mid x_{2}=0, x_{3}=0\right\}
$$

The set $\mathcal{G}=\operatorname{co}\left\{v_{1}, v_{2}\right\}$ and we note $\kappa=1, m=1$ and $\mathcal{B} \cap \operatorname{cone}(\mathcal{S})=\mathbf{0}$ thereby rendering RCP unsolvable using a continuous state feedback.

We verify that the necessary conditions for solution using piecewise affine feedback exist. Since $b_{1}:=(-2,-1,0) \in \mathcal{B} \cap \mathcal{C}_{1}, \mathcal{B}=\operatorname{span}\left\{b_{1}\right\}$ and $b_{2}:=-b_{1} \in \mathcal{B} \cap \mathcal{C}_{2}$, conditions (P3)-(P5) of Assumption 6.1 hold. It is clear (P6) also holds. Performing subdivision by Algorithm 6.2 we choose $v^{\prime}=(0.5,0.5,0) \in \overline{v_{0} v_{1}}$ such that we obtain the condition $\mathcal{B} \cap$ $\operatorname{cone}\left(\mathcal{S}_{1}\right) \neq 0$ where $\mathcal{S}_{1}:=\operatorname{co}\left\{v^{\prime}, v_{1}, v_{2}, v_{3}\right\}, \mathcal{S}_{2}:=\operatorname{co}\left\{v_{0}, v^{\prime}, v_{2}, v_{3}\right\}, \mathcal{F}_{0}=\operatorname{co}\left\{v^{\prime}, v_{2}, v_{3}\right\}$ and $h^{\prime}=(-0.5,1.5,0.5)$. The control inputs for $\mathcal{S}_{2}$ are $u_{0}=-1, u^{\prime}=-1, u_{22}=0$ and $u_{23}=0$. The control inputs for $\mathcal{S}_{1}$ are $u^{\prime}=-1, u_{11}=-2, u_{12}=0$ and $u_{13}=0$. This choice of control inputs yields the piecewise affine feedback law

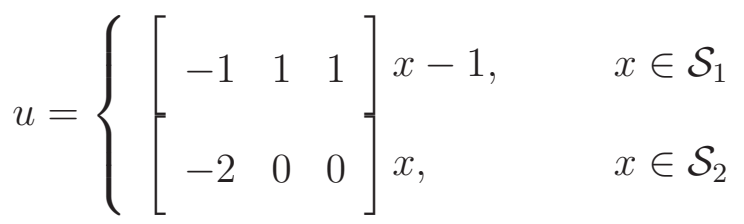

As shown below in Figures 7.11, 7.12 and 7.13, no equilibria occurs in $\mathcal{S}$ and the use of the piecewise feedback law allows all trajectories originating in $\mathcal{S}$ to exit via $\mathcal{F}_{0}$ rendering RCP solved.

The previous examples illustrated the use of piecewise affine feedback for solution of RCP in $\mathbb{R}^{2}$ and $\mathbb{R}^{3}$. In examples 7 - 10 we consider the use of a piecewise affine feedback solution for cases in higher dimensions. Importantly, these higher dimension examples demonstrate more clearly the use of the subdivision algorithm for instances where more than one iteration is required to eliminate the input deficiencies.

\subsubsection{Example 7}

Consider the simplex $\mathcal{S}$ in $\mathbb{R}^{4}$ defined by the following vertices $v_{0}=(0,0,0,0), v_{1}=$ $(1,0,0,0), v_{2}=(0,1,0,0), v_{3}=(0,0,1,0)$ and $v_{4}=(0,0,0,1)$. Consider also the affine 


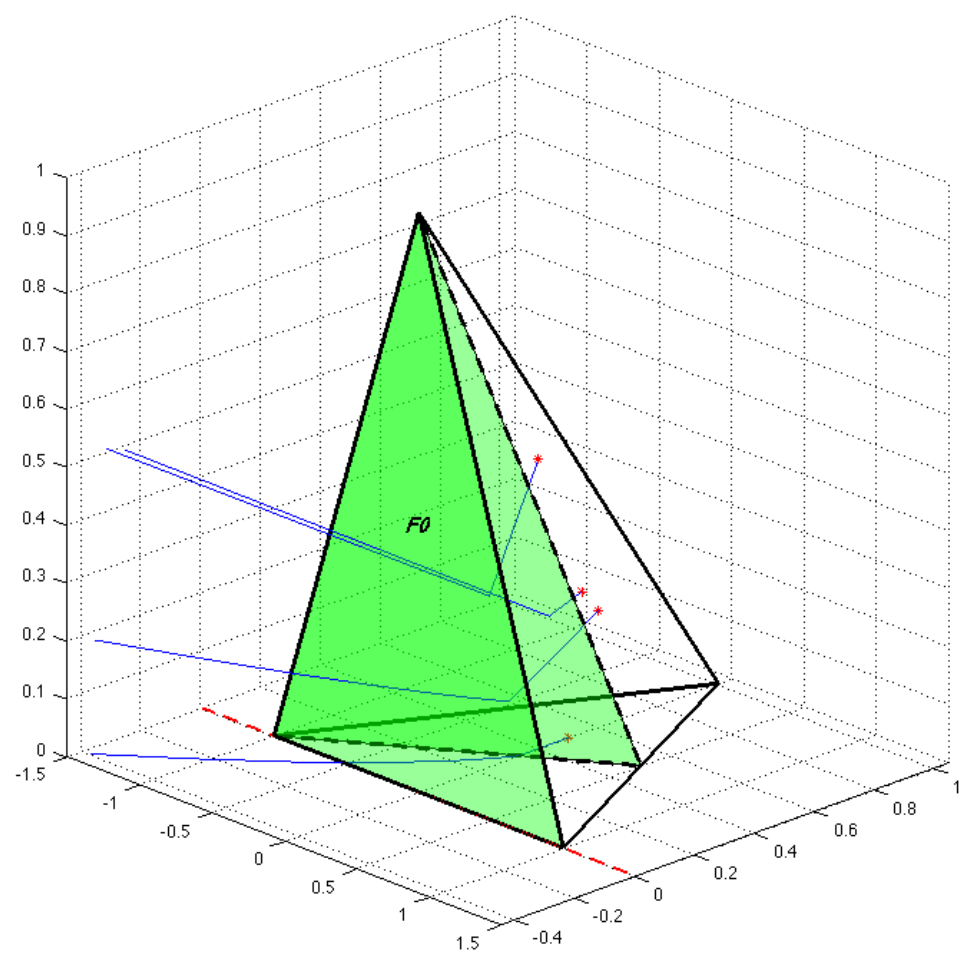

Figure 7.11: Example 6: Trajectories of initial conditions in $\mathcal{S}$ under piecewise affine feedback (view 1).

dynamics on $\mathcal{S}$

$$
\dot{x}=\left[\begin{array}{rrrr}
-3 & -3 & -3 & 1 \\
0 & 0 & 0 & -2 \\
-3 & -3 & -3 & 1 \\
0 & 0 & 0 & -2
\end{array}\right] x+\left[\begin{array}{rr}
0 & -2 \\
0 & 1 \\
-2 & 0 \\
1 & 0
\end{array}\right] u+\left[\begin{array}{l}
1 \\
1 \\
1 \\
1
\end{array}\right] .
$$

We have

$$
\mathcal{O}=\left\{x \mid x_{1}+x_{2}+x_{3}+x_{4}-1=0\right\}
$$

Hence $\mathcal{G}=\mathcal{S} \cap \mathcal{O}=\operatorname{co}\left\{v_{1}, v_{2}, v_{3}, v_{4}\right\}=\mathcal{F}_{0}$, with $\kappa=3$ and $m=2$. It can be easily shown that $\mathcal{B} \cap \operatorname{cone}(\mathcal{S})=\mathbf{0}$. With $\kappa>m$ and $\mathcal{B} \cap \operatorname{cone}(\mathcal{S})=\mathbf{0}$, by Theorem $5.3 \mathrm{RCP}$ is not solvable using a continuous state feedback. A piecewise affine feedback can be used to solve the problem.

We show briefly that the necessary conditions for solution using piecewise affine feed- 


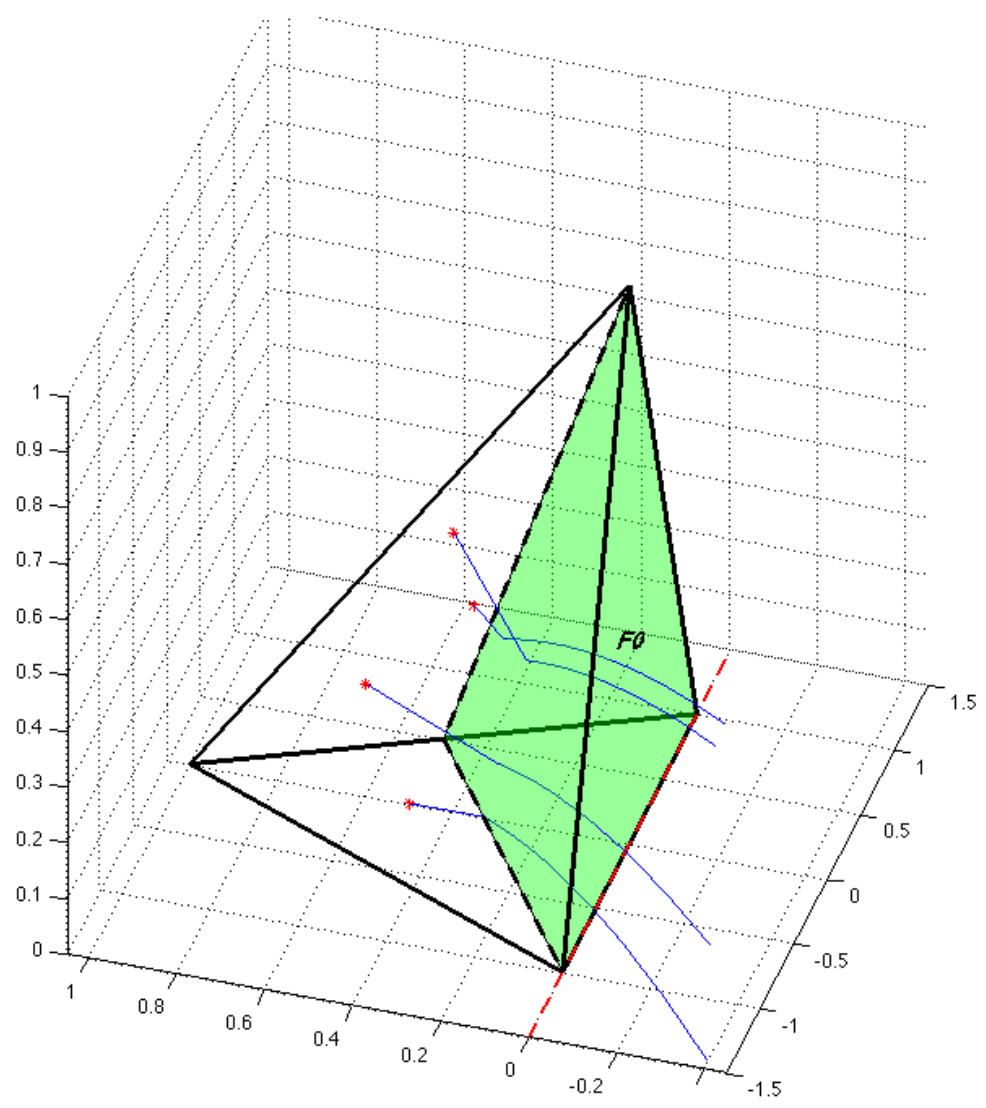

Figure 7.12: Example 6: Trajectories of initial conditions in $\mathcal{S}$ under piecewise affine feedback (view 2).

back hold. By inspection it is revealed $b_{1}:=(-2,1,0,0) \in \mathcal{B} \cap \mathcal{C}_{1}, b_{3}:=(0,0,2,-1) \in$ $\mathcal{B} \cap \mathcal{C}_{3}$ and $\mathcal{B}=\operatorname{span}\left\{b_{1}, b_{3}\right\} . \mathcal{B}$ splits into two dependent cycles with respect to $\mathcal{G}$, with $b_{2}:=-b_{1} \in \mathcal{B} \cap \mathcal{C}_{2}$ and $b_{4}:=-b_{3} \in \mathcal{B} \cap \mathcal{C}_{4}$. This shows that conditions (P3)-(P5) of Assumption 6.1 hold (although the indices have not been re-ordered as is the convention in the associated proofs). It is also immediately clear that (P6) holds.

\section{First Subdivision}

Under the initial iteration $\mathcal{S}$ is subdivided into $\mathcal{S}_{1}$ and $\mathcal{S}^{\prime}$. We find that $b_{2} \cdot h_{0}>0$ and therefore we choose $v^{\prime}=(0,0.75,0,0) \in \overline{v_{0} v_{2}}$ such that we obtain the condition $\mathcal{B} \cap \operatorname{cone}\left(\mathcal{S}_{1}\right) \neq 0$. Hence $\mathcal{S}_{1}:=\operatorname{co}\left\{v^{\prime}, v_{1}, v_{2}, v_{3}, v_{4}\right\}$ and $\mathcal{S}^{\prime}:=\operatorname{co}\left\{v_{0}, v_{1}, v^{\prime}, v_{3}, v_{4}\right\}$. Also $\mathcal{F}_{0}^{\prime}=\operatorname{co}\left\{v_{1}, v^{\prime}, v_{3}, v_{4}\right\}$ and $h^{\prime}=(-1,-1.33,-1,-1)$. It can be verified that $\mathcal{B} \cap$ 


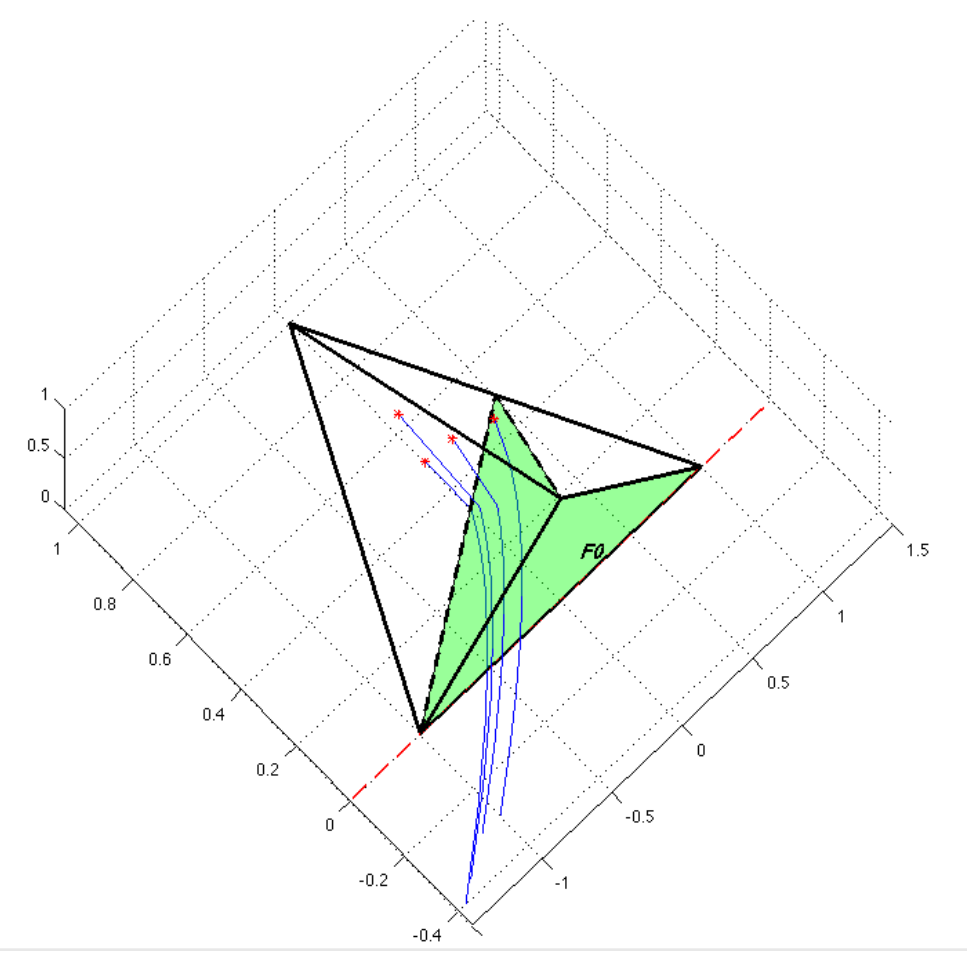

Figure 7.13: Example 6: Trajectories of initial conditions in $\mathcal{S}$ under piecewise affine feedback (view 3).

$\operatorname{cone}\left(\mathcal{S}_{1}\right) \neq 0$.

In order to satisfy the invariance conditions for $\mathcal{S}_{1}$ the control inputs at the vertices can be chosen as $u^{\prime}=(-1,-2), u_{11}=(-1,-2), u_{12}=(-1,-2), u_{13}=(-1,-2)$ and $u_{14}=(1,0)$. The resulting affine feedback law is

$$
u=\left[\begin{array}{llll}
0 & 0 & 0 & 2 \\
0 & 0 & 0 & 2
\end{array}\right] x+\left[\begin{array}{r}
-1 \\
-2
\end{array}\right] \quad x \in \mathcal{S}_{1}
$$

For $\mathcal{S}_{1}$ since the invariance conditions are solvable and $\mathcal{B} \cap \operatorname{cone}\left(\mathcal{S}_{1}\right) \neq \mathbf{0}$, by Theorem 4.2 RCP on $\mathcal{S}_{1}$ is solved. For $\mathcal{S}^{\prime}$ however, $\mathcal{G}^{\prime}=$ co $\left\{v_{1}, v_{3}, v_{4}\right\}$. Since $\kappa^{\prime}=2$ and $m=2$ by Theorem 5.3 RCP is not solvable on $\mathcal{S}^{\prime}$ by a continuous state feedback and further subdivision of $\mathcal{S}^{\prime}$ becomes necessary. 


\section{Second Subdivision}

Consider the sub-simplex $\mathcal{S}^{\prime}:=\operatorname{co}\left\{v_{0}, v_{1}, v^{\prime}, v_{3}, v_{4}\right\}$, where $v^{\prime}=(0,0.75,0,0) \in \overline{v_{0} v_{2}}$ and exit facet $\mathcal{F}_{0}^{\prime}=\operatorname{co}\left\{v_{1}, v^{\prime}, v_{3}, v_{4}\right\}$. Also the outward normal to the exit facet is $-h^{\prime}=(1,1.33,1,1)$. We divide $\mathcal{S}^{\prime}$ into $\mathcal{S}_{2}$ and $\mathcal{S}_{3}$ and solve RCP on $\mathcal{S}^{\prime}$ using a piecewise affine feedback. It is clear that $b_{4} \cdot\left(-h^{\prime}\right)>0$ and therefore we can choose $v^{\prime \prime} \in \overline{v_{0} v_{4}}$ such that we obtain the condition $\mathcal{B} \cap \operatorname{cone}\left(\mathcal{S}_{2}\right) \neq \mathbf{0}$. Let $v^{\prime \prime}=(0,0,0,0.8)$. We have $\mathcal{S}_{2}:=\operatorname{co}\left\{v^{\prime \prime}, v_{1}, v^{\prime}, v_{3}, v_{4}\right\}$ and $\mathcal{S}_{3}:=\operatorname{co}\left\{v_{0}, v_{1}, v^{\prime}, v_{3}, v^{\prime \prime}\right\}$. It can be verified that $\mathcal{B} \cap$ cone $\left(\mathcal{S}_{2}\right) \neq 0$. In order to satisfy the invariance conditions for $\mathcal{S}_{2}$ the control inputs at the vertices of $\mathcal{S}_{2}$ can be chosen as $u^{\prime \prime}=(-4,0.6), u_{21}=(-5,-1), u^{\prime}=(-1,-2)$, $u_{23}=(-5,-1)$ and $u_{24}=(-3,1)$. For $\mathcal{S}_{3}$ the control inputs at the vertices of $\mathcal{S}_{3}$ are chosen as $u_{0}=(0,0), u_{31}=(-1,0), u^{\prime}=(-1,-2), u_{33}=(0,-1)$ and $u^{\prime \prime}=(-4,0.6)$. This yields a piecewise affine feedback law

$$
u=\left\{\begin{array}{cccc}
{\left[\begin{array}{cccc}
3 & 9.33 & 3 & 5 \\
0 & -1.33 & 0 & 2
\end{array}\right] x+\left[\begin{array}{l}
-8 \\
-1
\end{array}\right],} & x \in \mathcal{S}_{2} \\
-1 & -1.33 & 0 & -5 \\
0 & -2.66 & -1 & 0.75
\end{array}\right] x, \quad x \in \mathcal{S}_{3}
$$

Since for $\mathcal{S}_{2}$ the invariance conditions are solvable and $\mathcal{B} \cap \operatorname{cone}\left(\mathcal{S}_{2}\right) \neq 0, \mathrm{RCP}$ on $\mathcal{S}_{2}$ is solved. Also for $\mathcal{S}_{3}$ we have $\mathcal{G}_{3}=\mathcal{S}_{3} \cap \mathcal{O}=$ co $\left\{v_{1}, v_{3}\right\}$. With $\kappa_{3}=1$ and $m=2$ we have a linearly independent set $\left\{b_{1}, b_{3} \mid b_{i} \in \mathcal{B} \cap C_{i}\right\}$ associated with $\mathcal{G}_{3}$. By Theorem 4.3 RCP on $\mathcal{S}_{3}$ is solvable by an affine feedback. Overall RCP on $\mathcal{S}$ is solved using a piecewise affine feedback

\subsubsection{Example 8}

Let $\mathcal{S}$ in $\mathbb{R}^{5}$ be defined by the following vertices $v_{0}=(0,0,0,0,0), v_{1}=(1,0,0,0,0)$, $v_{2}=(0,1,0,0,0)$ and $v_{3}=(0,0,1,0,0), v_{4}=(0,0,0,1,0)$ and $v_{5}=(0,0,0,0,1)$. The 
affine dynamics defined on $\mathcal{S}$ are

$$
\dot{x}=\left[\begin{array}{ccccc}
-1 & -1 & -1 & -1 & -1 \\
-1 & -1 & -1 & -1 & -1 \\
-1 & -1 & -1 & -1 & -1 \\
-1 & -1 & -1 & -1 & -1 \\
-1 & -1 & -1 & -1 & -1
\end{array}\right] x+\left[\begin{array}{ccc}
2 & 0 & 0 \\
1 & 0 & 0 \\
0 & -2 & 0 \\
0 & 1 & -2 \\
0 & 0 & 1
\end{array}\right] u+\left[\begin{array}{l}
1 \\
1 \\
1 \\
1 \\
1
\end{array}\right]
$$

We have

$$
\mathcal{O}=\left\{x \mid x_{1}+x_{2}+x_{3}+x_{4}+x_{5}-1=0\right\}
$$

Now $\mathcal{G}=\mathcal{S} \cap \mathcal{O}=\operatorname{co}\left\{v_{1}, v_{2}, v_{3}, v_{4}, v_{5}\right\}=\mathcal{F}_{0}$. Thus we have $\kappa=4$ and $m=3$. In addition it can be shown that $\mathcal{B} \cap \operatorname{cone}(\mathcal{S})=\mathbf{0}$, so RCP is not solvable using a continuous state feedback. Consider the piecewise affine feedback solution.

We show that the necessary conditions (P3)-(P6) of Assumption 6.1 hold. We find by inspection $b_{1}:=(-2,1,0,0,0) \in \mathcal{B} \cap \mathcal{C}_{1}, b_{3}:=(0,0,-2,1,0) \in \mathcal{B} \cap \mathcal{C}_{3}, b_{5}:=$ $(0,0,0,2,-1) \in \mathcal{B} \cap \mathcal{C}_{5}$ and $\mathcal{B}=\operatorname{span}\left\{b_{1}, b_{3}, b_{5}\right\} . \mathcal{B}$ splits into two dependent cycles with respect to $\mathcal{G}$, where $b_{2}:=-b_{1} \in \mathcal{B} \cap \mathcal{C}_{2}$ and $b_{4}:=-b_{3} \in \mathcal{B} \cap \mathcal{C}_{4}$. This shows that conditions (P3)-(P5) of Assumption 6.1 hold. It is immediately verified that (P6) holds.

\section{First subdivision}

Under the initial iteration $\mathcal{S}$ is subdivided into $\mathcal{S}_{1}$ and $\mathcal{S}^{\prime}$. Since $b_{2} \cdot h_{0}>0$ we choose $v^{\prime}=$ $(0,0.75,0,0,0) \in \overline{v_{0} v_{2}}$ such that we obtain the condition $\mathcal{B} \cap \operatorname{cone}\left(\mathcal{S}_{1}\right) \neq 0$. Hence $\mathcal{S}_{1}:=$ co $\left\{v^{\prime}, v_{1}, v_{2}, v_{3}, v_{4}, v_{5}\right\}$ and $\mathcal{S}^{\prime}:=\operatorname{co}\left\{v_{0}, v_{1}, v^{\prime}, v_{3}, v_{4}, v_{5}\right\}$. Also $\mathcal{F}_{0}^{\prime}=\operatorname{co}\left\{v_{1}, v^{\prime}, v_{3}, v_{4}, v_{5}\right\}$ and $h^{\prime}=(-1,-1.33,-1,-1,-1)$. It can be verified that $\mathcal{B} \cap \operatorname{cone}\left(\mathcal{S}_{1}\right) \neq 0$. In order to satisfy the invariance conditions for $\mathcal{S}_{1}$ the control inputs at the vertices can be chosen as $u^{\prime}=(-1,0,0), u_{11}=(-1,0,0), u_{12}=(-1,0,0), u_{13}=(-1,0,0), u_{14}=(-1,0,0)$ and 
$u_{15}=(-1,0,0)$. The resulting affine feedback law is

$$
u=\left[\begin{array}{r}
-1 \\
0 \\
0
\end{array}\right] \quad x \in \mathcal{S}_{1}
$$

For $\mathcal{S}_{1}$ since the invariance conditions are solvable and $\mathcal{B} \cap$ cone $\left(\mathcal{S}_{1}\right) \neq 0$, by Theorem 4.2 $\mathrm{RCP}$ on $\mathcal{S}_{1}$ is solved. For $\mathcal{S}^{\prime}$ however we have $\mathcal{G}^{\prime}=\operatorname{co}\left\{v_{1}, v_{3}, v_{4}, v_{5}\right\}$ and hence $\kappa^{\prime}=3$, $m=3$. By Theorem 5.3 RCP is not solvable on $\mathcal{S}^{\prime}$ by continuous state feedback. Hence further subdivision of $\mathcal{S}^{\prime}$ is required.

\section{Second Subdivision}

Consider the sub-simplex obtained from the first iteration $\mathcal{S}^{\prime}:=\operatorname{co}\left\{v_{0}, v_{1}, v^{\prime}, v_{3}, v_{4}, v_{5}\right\}$, where $v^{\prime}=(0,0.75,0,0,0) \in \overline{v_{0} v_{2}}$ and exit facet $\mathcal{F}_{0}^{\prime}=\operatorname{co}\left\{v_{1}, v^{\prime}, v_{3}, v_{4}, v_{5}\right\}$. Also the outward normal to the exit facet is $-h^{\prime}=(1,1.33,1,1,1)$. We divide $\mathcal{S}^{\prime}$ into $\mathcal{S}_{2}$ and $\mathcal{S}_{3}$ in order to obtain a piecewise affine feedback for $\mathcal{S}^{\prime}$. It is clear that $b_{4} \cdot\left(-h^{\prime}\right)>0$ and therefore we can choose $v^{\prime \prime} \in \overline{v_{0} v_{4}}=(0,0,0,0.8,0)$ such that we obtain the condition $\mathcal{B} \cap \operatorname{cone}\left(\mathcal{S}_{2}\right) \neq 0$. Thus $\mathcal{S}_{2}:=\operatorname{co}\left\{v^{\prime \prime}, v_{1}, v^{\prime}, v_{3}, v_{4}, v_{5}\right\}$ and $\mathcal{S}_{3}:=\operatorname{co}\left\{v_{0}, v_{1}, v^{\prime}, v_{3}, v^{\prime \prime}, v_{5}\right\}$. It can be verified that $\mathcal{B} \cap \operatorname{cone}\left(\mathcal{S}_{2}\right) \neq 0$. In order to satisfy the invariance conditions for $\mathcal{S}_{2}$ the control inputs at the vertices of $\mathcal{S}_{2}$ can be chosen as $u^{\prime \prime}=(0,-1,0), u_{21}=(0,-1,0)$, $u^{\prime}=(-1,0,0), u_{23}=(0,-1,0), u_{24}=(0,-1,0)$ and $u_{25}=(0,-1,0)$. In order to satisfy the invariance conditions for $\mathcal{S}_{3}$ the control inputs at the vertices of $\mathcal{S}_{3}$ can be chosen as $u_{0}=(0,0,0), u_{31}=(1,0,0), u^{\prime}=(-1,0,0), u_{33}=(0,1,0), u^{\prime \prime}=(0,-1,0)$ and 
$u_{35}=(0,0,-1)$. This yields a piecewise affine feedback law

$$
u=\left\{\begin{array}{ccccc}
0 & -1.33 & 0 & 0 & 0 \\
0 & 1.33 & 0 & 0 & 0 \\
0 & 0 & 0 & 0 & 0
\end{array}\right] x+\left[\begin{array}{r}
0 \\
-1 \\
0
\end{array}\right], \quad x \in \mathcal{S}_{2}
$$

For $\mathcal{S}_{2}$ the invariance conditions are solvable and $\mathcal{B} \cap \operatorname{cone}\left(\mathcal{S}_{2}\right) \neq \mathbf{0}$, so $\mathrm{RCP}$ on $\mathcal{S}_{2}$ is solved. For $\mathcal{S}_{3}$ we have $\mathcal{G}_{3}=\mathcal{S}_{3} \cap \mathcal{O}=$ co $\left\{v_{1}, v_{3}, v_{5}\right\}$. With $\kappa_{3}=2$ and $m=3$ RCP for $\mathcal{S}_{3}$ is solvable using affine feedback since $\left\{b_{1}, b_{3}, b_{5} \mid b_{i} \in \mathcal{B} \cap C_{i}\right\}$ is a linearly independent set associated with $\mathcal{G}_{3}$. Overall RCP on $\mathcal{S}$ is solved using the piecewise affine feedback.

\subsubsection{Example 9}

Define $\mathcal{S}$ in $\mathbb{R}^{7}$ by the following vertices $v_{0}=(0,0,0,0,0,0,0), v_{1}=(1,0,0,0,0,0,0), v_{2}=$ $(0,1,0,0,0,0,0)$ and $v_{3}=(0,0,1,0,0,0,0), v_{4}=(0,0,0,1,0,0,0), v_{5}=(0,0,0,0,1,0,0)$, $v_{6}=(0,0,0,0,0,1,0)$ and $v_{7}=(0,0,0,0,0,0,1)$ Also define the affine dynamics on $\mathcal{S}$ as

$$
\dot{x}=\left[\begin{array}{rrrrrrr}
-1 & -1 & -1 & -1 & -1 & -1 & -1 \\
-1 & -1 & -1 & -1 & -1 & -1 & -1 \\
-1 & -1 & -1 & -1 & -1 & -1 & -1 \\
-1 & -1 & -1 & -1 & -1 & -1 & -1 \\
-1 & -1 & -1 & -1 & -1 & -1 & -1 \\
-1 & -1 & -1 & -1 & -1 & -1 & -1 \\
0 & 0 & 0 & 0 & 0 & 0 & 1
\end{array}\right] x+\left[\begin{array}{rrr}
-2 & 0 & 0 \\
1 & 0 & 0 \\
0 & -2 & 0 \\
0 & 1 & 0 \\
0 & 0 & -2 \\
0 & 0 & 1 \\
0 & 0 & 0
\end{array}\right] u+\left[\begin{array}{l}
1 \\
1 \\
1 \\
1 \\
1 \\
1 \\
0
\end{array}\right]
$$

The set of points where $A x+a \in \mathcal{B}$ is defined as

$$
\mathcal{O}=\left\{x \mid x_{1}+x_{2}+x_{3}+x_{4}+x_{5}+x_{6}+x_{7}-1=0, x_{7}=0\right\}
$$


We have $\mathcal{G}=\mathcal{S} \cap \mathcal{O}=\operatorname{co}\left\{v_{1}, v_{2}, v_{3}, v_{4}, v_{5}, v_{6}\right\}, \kappa=5$ and $m=3$ and $\mathcal{B} \cap \operatorname{cone}(\mathcal{S})=\mathbf{0}$. RCP is not solvable using a continuous state feedback and a piecewise affine feedback using four individual controllers can be applied instead to solve the problem.

We have $b_{1}:=(-2,1,0,0,0,0,0) \in \mathcal{B} \cap \mathcal{C}_{1}, b_{3}:=(0,0,-2,1,0,0,0) \in \mathcal{B} \cap \mathcal{C}_{3}, b_{5}:=$ $(0,0,0,0,-2,1,0) \in \mathcal{B} \cap \mathcal{C}_{5}$ and $\mathcal{B}=\operatorname{span}\left\{b_{1}, b_{3}, b_{5}\right\}$. With three dependent vectors, $\mathcal{B}$ splits into three dependent cycles with respect to $\mathcal{G}$. Specifically we have $b_{2}:=-b_{1} \in$ $\mathcal{B} \cap \mathcal{C}_{2}, b_{4}:=-b_{3} \in \mathcal{B} \cap \mathcal{C}_{4}, b_{6}:=-b_{5} \in \mathcal{B} \cap \mathcal{C}_{6}$ This verifies that conditions (P3)-(P5) of Assumption 6.1 hold while it is immediately verified that (P6) holds.

\section{First subdivision}

Under the first iteration of the algorithm we subdivide $\mathcal{S}$ into $\mathcal{S}_{1}$ and $\mathcal{S}^{\prime}$. Since $b_{2} \cdot h_{0}>0$ we let $v^{\prime}=(0,0.75,0,0,0,0,0) \in \overline{v_{0} v_{2}}$ such that we obtain the condition $\mathcal{B} \cap \operatorname{cone}\left(\mathcal{S}_{1}\right) \neq$ 0 where $\mathcal{S}_{1}=\operatorname{co}\left\{v^{\prime}, v_{1}, v_{2}, v_{3}, v_{4}, v_{5}, v_{6}, v_{7}\right\}, \mathcal{S}^{\prime}=\operatorname{co}\left\{v_{0}, v_{1}, v^{\prime}, v_{3}, v_{4}, v_{5}, v_{6}, v_{7}\right\}, \mathcal{F}_{0}^{\prime}=$ co $\left\{v_{1}, v^{\prime}, v_{3}, v_{4}, v_{5}, v_{6}, v_{7}\right\}$ and $h^{\prime}=(-1,-1.33,-1,-1,-1,-1,-1)$. For satisfying invariance conditions for $\mathcal{S}_{1}$ the control inputs at the vertices can be chosen as $u^{\prime}=$ $(-1,0,0), u_{11}=(-1,0,0), u_{12}=(-1,0,0), u_{13}=(-1,0,0), u_{14}=(-1,0,0), u_{15}=$ $(-1,0,0), u_{16}=(-1,0,0)$ and $u_{17}=(0,0,0)$. The resulting affine feedback law is

$$
u=\left[\begin{array}{lllllll}
0 & 0 & 0 & 0 & 0 & 0 & 1 \\
0 & 0 & 0 & 0 & 0 & 0 & 0 \\
0 & 0 & 0 & 0 & 0 & 0 & 0
\end{array}\right] x+\left[\begin{array}{r}
-1 \\
0 \\
0
\end{array}\right] \quad x \in \mathcal{S}_{1}
$$

For $\mathcal{S}_{1}$ invariance conditions are solvable and $\mathcal{B} \cap \operatorname{cone}\left(\mathcal{S}_{1}\right) \neq \mathbf{0}, \mathrm{RCP}$ on $\mathcal{S}_{1}$ is solved. Since $\mathcal{G}^{\prime}=\operatorname{co}\left\{v_{1}, v_{3}, v_{4}, v_{5}, v_{6}\right\}$ then $\kappa^{\prime}=4$ and $m=3$ and RCP is not solvable on $\mathcal{S}^{\prime}$ by an affine feedback. Further subdivision of $\mathcal{S}^{\prime}$ is required.

\section{Second subdivision}

Consider $\mathcal{S}^{\prime}:=\operatorname{co}\left\{v_{0}, v_{1}, v^{\prime}, v_{3}, v_{4}, v_{5}, v_{6}, v_{7}\right\}$, with an exit facet $\mathcal{F}_{0}^{\prime}=\operatorname{co}\left\{v_{1}, v^{\prime}, v_{3}, v_{4}, v_{5}, v_{6}, v_{7}\right\}$ and corresponding outward normal $-h^{\prime}=(1,1.33,1,1,1,1,1)$. We divide $\mathcal{S}^{\prime}$ again into 
$\mathcal{S}_{2}$ and a new $\mathcal{S}^{\prime}$. Since $b_{4} \cdot\left(-h^{\prime}\right)>0$ we let $v^{\prime \prime} \in \overline{v_{0} v_{4}}=(0,0,0,0.8,0,0,0)$ such that we obtain the condition $\mathcal{B} \cap \operatorname{cone}\left(\mathcal{S}_{2}\right) \neq 0$ where $\mathcal{S}_{2}=\operatorname{co}\left\{v^{\prime \prime}, v_{1}, v^{\prime}, v_{3}, v_{4}, v_{5}, v_{6}, v_{7}\right\}$, $\mathcal{S}^{\prime}=\operatorname{co}\left\{v_{0}, v_{1}, v^{\prime}, v_{3}, v^{\prime \prime}, v_{5}, v_{6}, v_{7}\right\}$ and $h^{\prime}=(-1,-1.33,-1,-1.25,-1,-1,-1)$. In order to satisfy the invariance conditions for $\mathcal{S}_{2}$ the control inputs at the vertices of $\mathcal{S}_{2}$ can be chosen as $u^{\prime \prime}=(0,-1,0), u_{21}=(0,-1,0), u^{\prime}=(-1,0,0), u_{23}=(0,-1,0)$ $, u_{24}=(0,-1,0), u_{25}=(0,-1,0), u_{26}=(0,-1,0)$ and $u_{27}=(0,0,0)$. We obtain an affine feedback law

$$
u=\left[\begin{array}{rrrrrrr}
0 & -1.33 & 0 & 0 & 0 & 0 & 0 \\
0 & 1.33 & 0 & 0 & 0 & 0 & 1 \\
0 & 0 & 0 & 0 & 0 & 0 & 0
\end{array}\right] x+\left[\begin{array}{c}
0 \\
1 \\
0
\end{array}\right] x \in \mathcal{S}_{2}
$$

With $\mathcal{S}_{2}$ we have the invariance conditions holding and $\mathcal{B} \cap$ cone $\left(\mathcal{S}_{2}\right) \neq \mathbf{0}$, guaranteeing solution of RCP. For $\mathcal{S}^{\prime}$ we have $\mathcal{G}^{\prime}=\operatorname{co}\left\{v_{1}, v_{3}, v_{5}, v_{6}\right\}$ and hence $\kappa^{\prime}=3$ and $m=3$ so RCP is not solvable on $\mathcal{S}^{\prime}$ therefore eliciting another subdivision of $\mathcal{S}^{\prime}$.

\section{Third subdivision}

The previous subdivision yields $\mathcal{S}^{\prime}=\operatorname{co}\left\{v_{0}, v_{1}, v^{\prime}, v_{3}, v^{\prime \prime}, v_{5}, v_{6}, v_{7}\right\}$ with $\mathcal{F}_{0}^{\prime}=\operatorname{co}\left\{v_{1}, v^{\prime}, v_{3}, v^{\prime \prime}, v_{5}, v_{6}, v_{7}\right\}$ and $-h^{\prime}=(1,1.33,1,1.25,1,1,1)$. Dividing $\mathcal{S}^{\prime}$ once again into sub-simplices $\mathcal{S}_{3}$ and $\mathcal{S}_{4}$, we have $b_{6} \cdot\left(-h^{\prime}\right)>0$ and therefore we can choose $v^{\prime \prime \prime}=(0,0,0,0,0,0.8,0) \in$ $\overline{v_{0} v_{6}}$. Hence $\mathcal{B} \cap \operatorname{cone}\left(\mathcal{S}_{3}\right) \neq 0$ where $\mathcal{S}_{3}=\operatorname{co}\left\{v^{\prime \prime \prime}, v_{1}, v^{\prime}, v_{3}, v^{\prime \prime}, v_{5}, v_{6}, v_{7}\right\}$ and $\mathcal{S}_{4}=$ co $\left\{v_{0}, v_{1}, v^{\prime}, v_{3}, v^{\prime \prime}, v_{5}, v^{\prime \prime \prime}, v_{7}\right\}$.In order to satisfy the invariance conditions for $\mathcal{S}_{3}$ the control inputs can be chosen as $u^{\prime \prime \prime}=(0,0,-1), u_{31}=(0,0,-1), u^{\prime}=(-1,0,0)$, $u_{33}=(0,0,-1), u^{\prime \prime}=(0,-1,0), u_{35}=(0,0,-1), u_{36}=(0,0,-1)$ and $u_{37}=(0,0,0)$. For $\mathcal{S}_{4}$ we use: $u_{0}=(0,0,0), u_{41}=(1,0,0), u^{\prime}=(-1,0,0), u_{43}=(0,1,0), u^{\prime \prime}=(0,-1,0)$, $u_{45}=(0,0,1), u^{\prime \prime \prime}=(0,0,-1)$ and $u_{47}=(0,0,0)$. Finally the resulting piecewise affine 
feedback law for $\mathcal{S}^{\prime}$ is

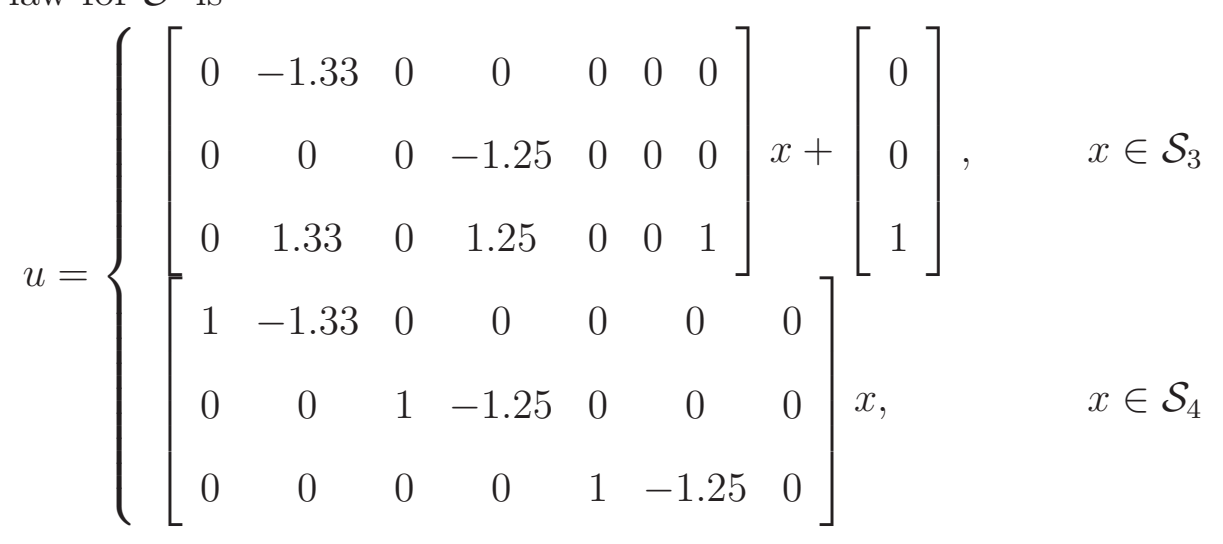

For $\mathcal{S}_{3}$ the invariance conditions are solvable and $\mathcal{B} \cap \operatorname{cone}\left(\mathcal{S}_{3}\right) \neq \mathbf{0}$, so $\mathrm{RCP}$ on $\mathcal{S}_{3}$ is solved. For $\mathcal{S}_{4}$ we have $\mathcal{G}_{4}=\mathcal{S}_{4} \cap \mathcal{O}=$ co $\left\{v_{1}, v_{3}, v_{5}\right\}$. With $\kappa_{3}=2$ and $m=3 \mathrm{RCP}$ for $\mathcal{S}_{3}$ is solvable using affine feedback. We can verify $\left\{b_{1}, b_{3}, b_{5} \mid b_{i} \in \mathcal{B} \cap C_{i}\right\}$ is a linearly independent set associated with $\mathcal{G}_{3}$. With RCP solved for $\mathcal{S}_{1}, \mathcal{S}_{2}, \mathcal{S}_{3}$ and $\mathcal{S}_{4}$ each with an affine feedback, the overall RCP is solved on $\mathcal{S}$ with the piecewise affine law.

\subsubsection{Example 10}

Recall the simplex $\mathcal{S}$ in $\mathbb{R}^{7}$ as in Example 6. Consider however the new affine dynamics

$$
\dot{x}=\left[\begin{array}{rrrrrrr}
-1 & -1 & -1 & -1 & -1 & -1 & -1 \\
-1 & -1 & -1 & -1 & -1 & -1 & -1 \\
-1 & -1 & -1 & -1 & -1 & -1 & -1 \\
-1 & -1 & -1 & -1 & -1 & -1 & -1 \\
-1 & -1 & -1 & -1 & -1 & -1 & -1 \\
0 & 0 & 0 & 0 & 0 & 1 & 0 \\
0 & 0 & 0 & 0 & 0 & 0 & 1
\end{array}\right] x+\left[\begin{array}{rrr}
-2 & 0 & 0 \\
1 & 0 & 0 \\
0 & -2 & 0 \\
0 & 1 & -2 \\
0 & 0 & 1 \\
0 & 0 & 0 \\
0 & 0 & 0
\end{array}\right] u+\left[\begin{array}{l}
1 \\
1 \\
1 \\
1 \\
1 \\
0 \\
0
\end{array}\right] .
$$

With the new dynamics on $\mathcal{S}$ we have

$$
\mathcal{O}=\left\{x \mid x_{1}+x_{2}+x_{3}+x_{4}+x_{5}+x_{6}+x_{7}-1=0, x_{6}=0, x_{7}=0\right\}
$$

So $\mathcal{G}=\operatorname{co}\left\{v_{1}, v_{2}, v_{3}, v_{4}, v_{5}\right\}$ with $\kappa=4$ and $m=3$. Also $\mathcal{B} \cap \operatorname{cone}(\mathcal{S})=\mathbf{0}$. We show that the problem is solvable using a piecewise feedback since RCP is not solvable using continuous state feedback. 
It is clear (P6) of Assumption 6.1 holds. Also, by observation we find $b_{1}:=$ $(-2,1,0,0,0,0,0) \in \mathcal{B} \cap \mathcal{C}_{1}, b_{3}:=(0,0,-2,1,0,0,0) \in \mathcal{B} \cap \mathcal{C}_{3}, b_{4}:=(0,0,0,-2,1,0,0) \in$ $\mathcal{B} \cap \mathcal{C}_{4}$ and $\mathcal{B}=\operatorname{span}\left\{b_{1}, b_{3}, b_{4}\right\}$. Now $\mathcal{B}$ splits into two dependent cycles with respect to $\mathcal{G}$ where $b_{2}:=-b_{1} \in \mathcal{B} \cap \mathcal{C}_{2}$ and $b_{5}:=-b_{3}-b_{4} \in \mathcal{B} \cap \mathcal{C}_{5}$. Thus, conditions (P3)-(P5) of Assumption 6.1 also hold.

\section{First subdivision}

Dividing $\mathcal{S}$ into $\mathcal{S}_{1}$ and $\mathcal{S}^{\prime}$ we choose $v^{\prime} \in \overline{v_{0} v_{2}}=(0,0.75,0,0,0,0,0)$ such that we obtain the condition $\mathcal{B} \cap \operatorname{cone}\left(\mathcal{S}_{1}\right) \neq 0$ where $\mathcal{S}_{1}=$ co $\left\{v^{\prime}, v_{1}, v_{2}, v_{3}, v_{4}, v_{5}, v_{6}, v_{7}\right\}$ and $\mathcal{S}^{\prime}=$ co $\left\{v_{0}, v_{1}, v^{\prime}, v_{3}, v_{4}, v_{5}, v_{6}, v_{7}\right\}$. Note $h^{\prime}=(-1,-1.33,-1,-1,-1,-1,-1)$. For satisfying invariance conditions for $\mathcal{S}_{1}$ the control inputs at the vertices can be chosen as $u^{\prime}=$ $(-1,0,0), u_{11}=(-1,0,0), u_{12}=(-1,0,0), u_{13}=(-1,0,0), u_{14}=(-1,0,0), u_{15}=$ $(-1,0,0), u_{16}=(0,0,0)$ and $u_{17}=(0,0,0)$. The resulting affine feedback law is

$$
u=\left[\begin{array}{lllllll}
0 & 0 & 0 & 0 & 0 & 1 & 1 \\
0 & 0 & 0 & 0 & 0 & 0 & 0 \\
0 & 0 & 0 & 0 & 0 & 0 & 0
\end{array}\right] x+\left[\begin{array}{r}
-1 \\
0 \\
0
\end{array}\right] x \in \mathcal{S}_{1} .
$$

Invariance conditions are solvable and $\mathcal{B} \cap \operatorname{cone}\left(\mathcal{S}_{1}\right) \neq \mathbf{0}$ so $\mathrm{RCP}$ on $\mathcal{S}_{1}$ is solved. $\mathcal{G}^{\prime}=\operatorname{co}\left\{v_{1}, v_{3}, v_{4}, v_{5}\right\}$ and hence $\kappa^{\prime}=3$ and $m=3$ implying RCP is not solvable on $\mathcal{S}^{\prime}$ by an affine feedback.

\section{Second subdivision}

We have $\mathcal{S}^{\prime}:=\operatorname{co}\left\{v_{0}, v_{1}, v^{\prime}, v_{3}, v_{4}, v_{5}, v_{6}, v_{7}\right\}$, with an exit facet $\mathcal{F}_{0}^{\prime}=\operatorname{co}\left\{v_{1}, v^{\prime}, v_{3}, v_{4}, v_{5}, v_{6}, v_{7}\right\}$ and corresponding outward normal $-h^{\prime}=(1,1.33,1,1,1,1,1)$. We divide $\mathcal{S}^{\prime}$ again into $\mathcal{S}_{2}$ and a new $\mathcal{S}_{3}$. Since $b_{5} \cdot\left(-h^{\prime}\right)>0$ we let $v^{\prime \prime} \in \overline{v_{0} v_{5}}=(0,0,0,0,0.8,0,0)$ such that we obtain the condition $\mathcal{B} \cap \operatorname{cone}\left(\mathcal{S}_{2}\right) \neq 0$ where $\mathcal{S}_{2}=\operatorname{co}\left\{v^{\prime \prime}, v_{1}, v^{\prime}, v_{3}, v_{4}, v_{5}, v_{6}, v_{7}\right\}$, $\mathcal{S}_{3}=\operatorname{co}\left\{v_{0}, v_{1}, v^{\prime}, v_{3}, v_{4}, v^{\prime \prime}, v_{6}, v_{7}\right\}$. In order to satisfy the invariance conditions for $\mathcal{S}_{2}$ the control inputs at the vertices of $\mathcal{S}_{2}$ can be chosen as $u^{\prime \prime}=(0,-2,-1), u_{21}=(0,-2,-1)$, 
$u^{\prime}=(-1,0,0), u_{23}=(0,-2,-1), u_{24}=(0,-2,-1), u_{25}=(0,-2,-1), u_{26}=(0,0,0)$ and $u_{27}=(0,0,0)$. For $\mathcal{S}_{3}$ we have $u_{0}=(0,0,0), u_{31}=(1,0,0), u^{\prime}=(-1,0,0), u_{33}=(0,1,0)$ $, u_{34}=(0,0,1), u^{\prime \prime}=(0,-2,-1), u_{36}=(0,0,0)$ and $u_{37}=(0,0,0)$. We obtain

$$
u=\left\{\begin{array}{ccccccc}
0 & -1.33 & 0 & 0 & 0 & 0 & 0 \\
0 & 2.66 & 0 & 0 & 0 & 2 & 2 \\
0 & 1.33 & 0 & 0 & 0 & 1 & 1
\end{array}\right] x+\left[\begin{array}{r}
0 \\
-2 \\
-1
\end{array}\right], \quad x \in \mathcal{S}_{2}
$$

For $\mathcal{S}_{2}$ the invariance conditions are solvable and $\mathcal{B} \cap \operatorname{cone}\left(\mathcal{S}_{2}\right) \neq \mathbf{0}$, so $\mathrm{RCP}$ on $\mathcal{S}_{2}$ is solved. For $\mathcal{S}_{3}$ we have $\mathcal{G}_{3}=\mathcal{S}_{3} \cap \mathcal{O}=$ co $\left\{v_{1}, v_{3}, v_{4}\right\}$. With $\kappa_{3}=2$ and $m=3 \mathrm{RCP}$ for $\mathcal{S}_{3}$ is solvable using affine feedback since and it can be seen $\left\{b_{1}, b_{3}, b_{4} \mid b_{i} \in \mathcal{B} \cap C_{i}\right\}$ is a linearly independent set associated with $\mathcal{G}_{3}$. Overall RCP on $\mathcal{S}$ is solved using the piecewise affine feedback.

\subsection{Further ideas on RCP solution}

The previous section described examples in which RCP was not solvable using a continuous state feedback. In these examples the conditions outlined in Assumption 6.1 which are necessary for solution of RCP using a piecewise affine feedback are shown to hold, and the subdivision algorithm is used to subdivide $\mathcal{S}$ for development of the control law. It is useful to investigate instances whereby continuous feedback cannot be used and the necessary condition (P6) of Assumption 6.1 fails. In such cases a subdivision of $\mathcal{S}$ into full dimensional simplices for a piecewise affine feedback which solves RCP is not possible. We briefly consider how the use of lower dimensional sub-simplices ,may or may not solve RCP on $\mathcal{S}$ in such cases. 


\subsubsection{Example 11}

Consider a simplex $\mathcal{S}$ determined by $v_{0}=(0,0.5,0.5), v_{1}=(1,0,0)$ and $v_{2}=(-1,0,0)$ and $v_{3}=(0,0,1)$ and consider the affine system

$$
\dot{x}=\left[\begin{array}{rrr}
0 & 0 & 0 \\
0 & -2 & -2 \\
0 & 0 & 1
\end{array}\right] x+\left[\begin{array}{l}
1 \\
0 \\
0
\end{array}\right] u+\left[\begin{array}{l}
0 \\
0 \\
0
\end{array}\right] .
$$

We have

$$
\mathcal{O}=\left\{x \mid x_{2}=0, x_{3}=0\right\}
$$

Hence $\mathcal{G}=\operatorname{co}\left\{v_{1}, v_{2}\right\}$ with $\kappa=1$ and $m=1$. Also $\mathcal{B} \cap \operatorname{cone}(\mathcal{S})=\mathbf{0}$ so RCP is not solvable. Moreover, (P6) is immediately shown to not hold and hence Theorem 6.1 cannot be used to gurantee solution using a piecewise affine feedback. Nonetheless we derive an affine feedback law and observe the characteristics of the resultant closed loop vector field. In order to satisfy the invariance conditions of $\mathcal{S}$ we choose the following control inputs at the vertices of $\mathcal{S}: u_{0}=0, u_{1}=-1, u_{2}=1$, and $u_{3}=0$. This results in the affine feedback law

$$
u=\left[\begin{array}{lll}
-1 & 0 & 0
\end{array}\right] x \quad x \in \mathcal{S} .
$$

Due to the orientation of the closed loop vector field within $\mathcal{S}$ we can see as in Figures 7.14 and 7.15 that all trajectories originating in $\mathcal{S}$ but outside of $\mathcal{G}$ will exit via $\mathcal{F}_{0}$. Only trajectories with initial conditions on $\mathcal{G}$ will be exposed to the equilibrium point. We therefore use a lower dimension simplex to mask the equilibrium point on $\mathcal{G}$. Let $\mathcal{S}_{1}=\mathcal{G}=\operatorname{co}\left\{v_{1}, v_{2}\right\}$, and $\mathcal{S}_{2}=\mathcal{S} \backslash \mathcal{G}$. We apply the original control law derived above for $\mathcal{S}$ to $\mathcal{S}_{2}$. For $\mathcal{S}_{1}$ however a control law such as $u=1$ can be used. The effect of using such a control law for $\mathcal{S}_{1}$ ensures that any trajectory starting on $\mathcal{G}$ will exit $\mathcal{F}_{0}$ in the direction of $\mathcal{B}$. All other trajectories originating in $\mathcal{S}_{2}$ will avoid the set $\mathcal{G}$ and exit $\mathcal{F}_{0}$ also, thus solving the problem. We have thus provided an example of how RCP may be solved in the event where continuous state feedback cannot and the condition (P6) does 
not hold. It is important to note however, that this solution does not satisfy condition (iii) of RCP, and the synthesis method outlined is not suitable in a design context where this condition must be strictly enforced.

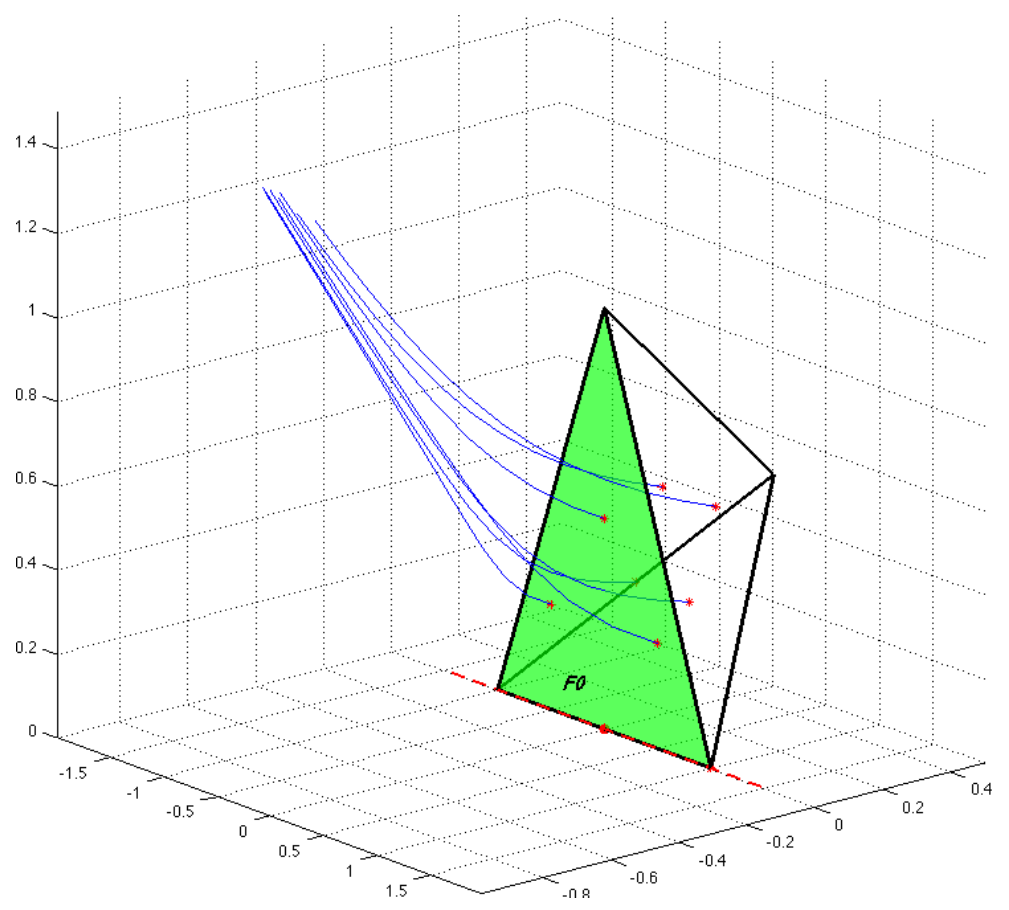

Figure 7.14: Example 11: View 1 showing trajectories with initial conditions not in $\mathcal{G}$ all leave $\mathcal{S}$.

\subsubsection{Example 12}

Example 11 documented how the use of a lower-dimension simplex solved RCP for instances where affine feedback and piecewise affine feedback (using full dimension simplices) cannot be used. We consider a counter example. Let $\mathcal{S}$ be determined by $v_{0}=(0,1), v_{1}=(1,0)$ and $v_{2}=(-1,0)$, and consider the affine system

$$
\dot{x}=\left[\begin{array}{rr}
0 & 0 \\
0 & -1
\end{array}\right] x+\left[\begin{array}{l}
1 \\
0
\end{array}\right] u+\left[\begin{array}{l}
0 \\
0
\end{array}\right] \text {. }
$$

We have

$$
\mathcal{O}=\left\{x \mid x_{2}=0\right\}
$$




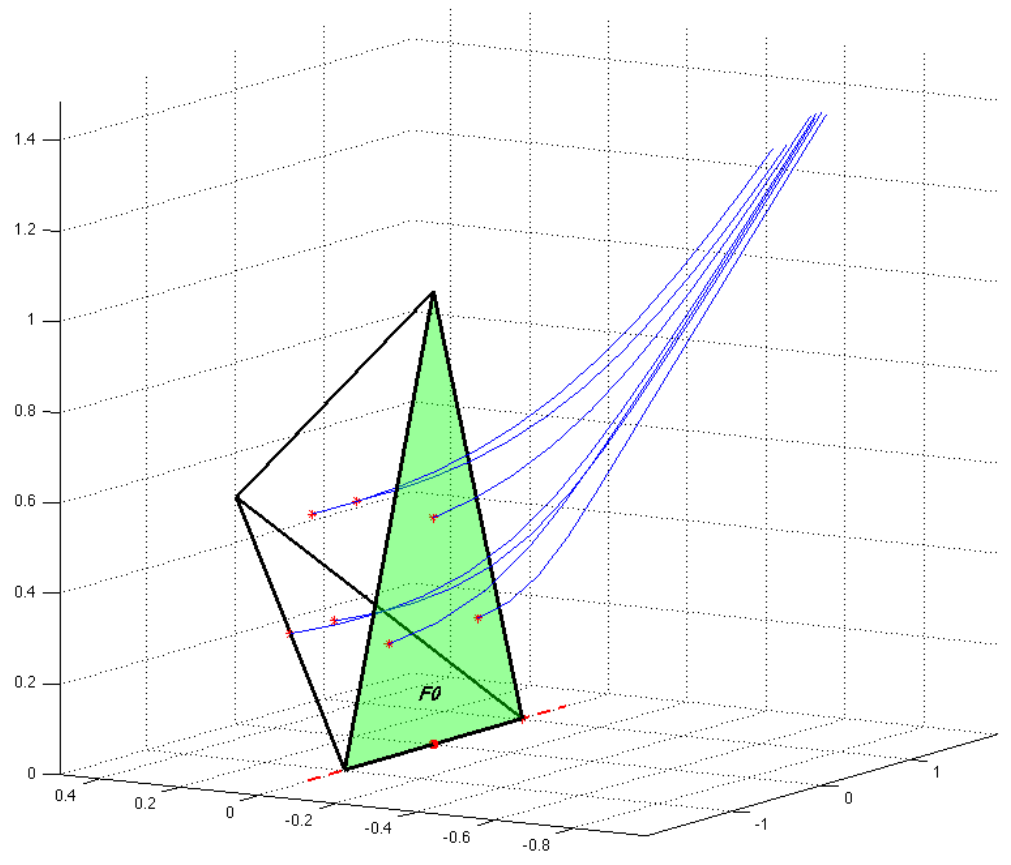

Figure 7.15: Example 11: View 2 showing trajectories with initial conditions not in $\mathcal{G}$ all leave $\mathcal{S}$.

Hence $\mathcal{G}=\operatorname{co}\left\{v_{1}, v_{2}\right\}$ with $\kappa=1, m=1$ and $\mathcal{B} \cap \operatorname{cone}(\mathcal{S})=\mathbf{0}$. By Theorem 5.3 , using continuous state feedback RCP is not solvable due to equilibria formation. Moreover, (P6) is immediately shown to not hold and hence theorem 6.1 cannot be used. We attempt to mask the equilibrium as carried out in Example 11 by using one control law for $\mathcal{G}$ and another for the rest of $\mathcal{S}$. To satisfy the invariance conditions for $\mathcal{S}$ we choose: $u_{0}=0, u_{1}=-1, u_{2}=1$. The affine feedback becomes.

$$
u=\left[\begin{array}{ll}
-1 & 0
\end{array}\right] x
$$

Simulation of the closed-loop system is shown in the following Figure 7.16. The vector field satisfies the invariance conditions however it is clear there exists an equilibrium point on the set $\mathcal{G}$ (the exit facet $\mathcal{F}_{0}$ ). Suppose we set $\mathcal{S}_{1}=\mathcal{G}=$ co $\left\{v_{1}, v_{2}\right\}$, and $\mathcal{S}_{2}=\mathcal{S} \backslash \mathcal{G}$. We apply the affine feedback law derived above for $\mathcal{S}$ to $\mathcal{S}_{2}$. For $\mathcal{S}_{1}$ however we use a control law such as $u=1$ can be used. Close inspection reveals that the vector field on $\mathcal{S}_{2}$ will be such that no closed loop trajectory starting in $\mathcal{S}_{2}$ will reach $\mathcal{S}_{1}$ in finite time. 
Hence this problem is not solvable whatsoever due to failure of (P6).

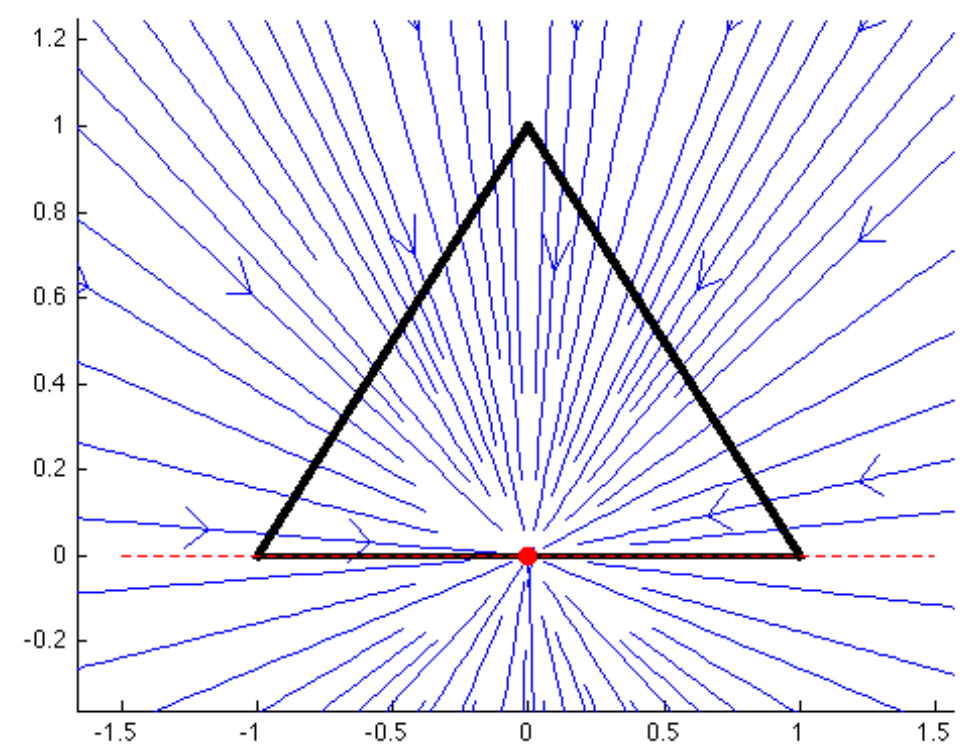

Figure 7.16: Example 12: Closed loop vector field on $\mathcal{S}$

The above two examples suggests that although the use of lower dimensional simplices may solve RCP, the effectiveness of the synthesis method may not be easily characterized, being very specific to the problem and depending highly on the interaction of the affine system and the simplex geometry. 


\section{Chapter 8}

\section{A Biomedical Application}

The thesis presents the development and solution of RCP on simplices, a specific problem which encapsulates the aspects of reachability for control systems. It is useful to illustrate the engineering design context in which the problem may arise, that is, where there exist specific state constraints and a clear requirement for trajectories to evolve in a precise and finite-time manner. We use the automation of anesthesia delivery to human patients for surgical procedures as a realistic platform to inform on the general idea of complex specifications and how the latter can realized by triangulation of the state space and using the reach problem formulated on simplices. Such insight stresses the importance of deep studies into RCP solution as provided in the thesis.

\subsection{Anesthesia Delivery and Control}

In recent years the field of anesthesia has witnessed breakthroughs in the monitoring of the anesthetic state prompting interest from the control community in the automation of clinical anesthesia [29]. Since human physiological processes demonstrate a relatively large degree of complexity, it is expected that an automation problem such as safe delivery of anesthesia for surgical procedures give rise to complex and precise specifications. 
During surgery, the control objective focuses on maintaining a deep level of hypnosis by sufficient infusion of anesthetic drug to the patient. This is known as anesthesia maintenance. However the process of transferring a fully awake patient to the steady state point where hypnosis levels are deep enough to perform surgery (anesthesia induction process) is equally important. During this transient period, it is reasonable to assume that state variables representative of anesthetic drug concentration in the body and/or important physiological variables, obey certain limitations or evolve according to a set of complex rules or specifications. Our focus is on the control design which realizes various examples of these complex control specifications which are not readily encoded in a typical steady-state or regulation-based problem.

We first introduce the typical compartmental modeling paradigm as summarized in [28] which is often used to describe the anesthetic input to physiological patient outputs such as Heart Rate(HR), Mean Arterial Blood Pressure (MAP) and BIS level (an indicator of the level of depth of anesthesia which is explained later). Pharmacokinetic (PK) compartment models are first used to predict the concentration of the drug in the body by modeling the diffusion rate of the drug in tissues and organs, and the flow of drug in the blood. Conceptual compartments represent groupings of body tissues and organs. Drug flows into each compartment from external anesthetic inputs or through transfer from other compartments or both. Drug flows out of each compartment via transfer to other compartments and metabolic elimination. Consider a typical three compartment model, represented by the following state equations:

$$
\begin{aligned}
& \dot{x_{1}}=I+k_{21} x_{2}+k_{31} x_{3}-k_{10} x_{1}-x_{1} k_{12}-x_{1} k_{13} \\
& \dot{x_{2}}=k_{12} x_{1}-k_{21} x_{2} \\
& \dot{x_{3}}=k_{13} x_{1}-k_{31} x_{3}
\end{aligned}
$$

$x_{i}$ represents drug concentration in the $i^{t h}$ compartment, $k_{i, j}$ is the distribution transfer rate from the $i^{\text {th }}$ compartment to the $j^{\text {th }}$ compartment, $k_{10}$ is the clearance transfer rate 
out of the central compartment, and $I$ represents anesthetic infusion rate into the central compartment. For inhaled anesthetic agents, the central compartment refers to the lungs and the central compartment concentration $x_{1}$ is known as the endtidal concentration, referring to alveoli concentrations at the end of a respiratory cycle. The second peripheral compartment may refer to a vessel-rich grouping of tissues and organs such as brain/liver while the third compartment may represent vessel-poor tissues such as bones and fatty tissue. Figure 8.1 describes conceptually this three-compartment pharmacokinetic model.

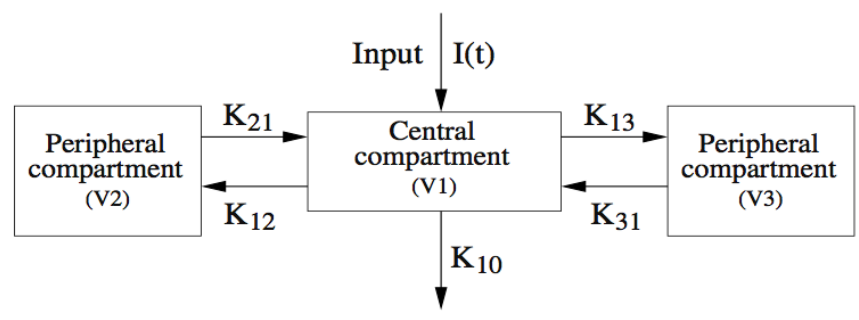

Figure 8.1: A three-compartment PK model

Pharmacodynamic (PD) compartment models on the other hand are used to describe the relationship between drug concentration and the clinical effects on the patient including BIS level, heart rate, blood pressure. The models, given by nonlinear functions, relate the magnitude of the pharmacological effect to the concentration of the drug at its site of action or effect site. In order to accurately construct these models, concentrations at the actual effect site are needed. Since effect site concentrations are not always easily observed, concentrations predicted from the PK model are used in order to determine the response from the input anesthetics to the effect of the drug. Hence an effect compartment is attached to the central compartment of the PK model to capture transport time to the effect site. To model time for drug transfer from the central compartment to the effect compartment we have:

$$
\dot{x_{e}}=k_{e 0}\left(x_{1}-x_{e}\right)
$$


$x_{1}$ represents concentration in the central compartment, $x_{e}$ represents concentrations in the effect compartment and $k_{e 0}$ is the associated rate constant. The most common PD structure used to characterize relationship between concentration and effect of anesthetic drug are functions with the general form :

$$
e=\frac{e_{\max } \cdot x_{e}^{\gamma}}{K_{50}^{\gamma}+x_{e}^{\gamma}}
$$

where $e$ is the measured effect such as heart rate, blood pressure or BIS, $K_{50}$ is the concentration of the drug at which half of the maximum achievable effect is observed in the patient, and $\gamma$ is used to better fit observed data. A block diagram summarizing the overall PK-PD structure is given in Figure 8.2.

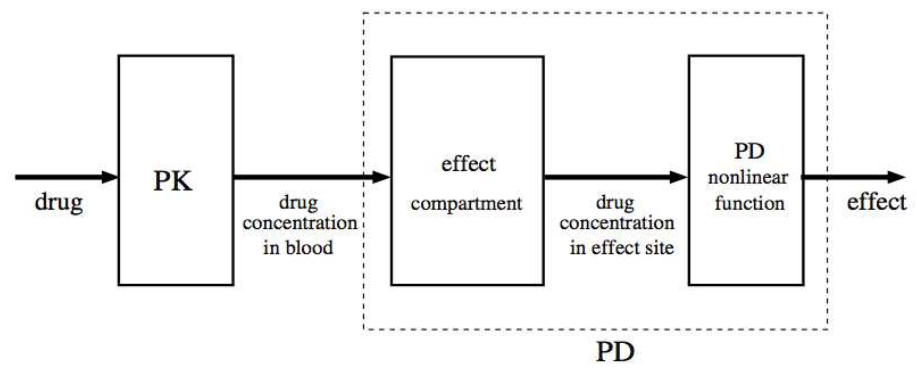

Figure 8.2: Overall PK-PD model

The fact that the brain can or cannot process sensory information can be observed in a machine known as the electroencephalogram (EEG), and an EEG derived parameter known as the bispectral index (BIS) can be used to clinically provide a continuous estimate of the hypnotic state of the patient [30]. BIS predicts accurately levels of consciousness and is well-correlated with depth of anesthesia [30, 32]. BIS values lie in the range $0-100$, where 100 corresponds to a fully conscious subject, while 0 corresponds to no EEG signal output coming from brain activity. A BIS value around 60 corresponds to a moderate hypnotic state, a BIS value around 40 corresponds to a deep hypnotic state, and very low BIS values are referred to as characterizing a profound anesthetic state [28]. In [33] a detailed study of the transient BIS variation during the anesthesia induction 
process is given and the data presented is useful in later determining state constraints for our complex control examples.

\subsection{A Full Model For BIS Control}

In [30] the authors have developed a system to control the anesthesia depth level, with BIS level as the controlled variable and the inhaled anesthetic agent isoflurane as the control input. The system is chosen as a suitable platform for illustrating our ideas on complex control. A complete model is given consisting of 1) a model of the closed circuit respiratory system which delivers the anesthetic agent isoflurane via patient inhalation; 2) the PK model describing the drug distribution within the body and 3) the PD model describing the time course of the BIS.

First consider the model and description of the closed circuit respiratory system. The schematic representation is shown in Figure 8.3. The pump moves the breathing mixture (consisting of air and isoflurane) into the subject's lungs and stays at rest during exhalation. The breathing machine ensures proper circulation of gases in the system and prevent patients from rebreathing exhaled gas. Fresh gas flow entering the machine is denoted by $Q_{0}$ and its anesthetic concentration $C_{0}$ is the manipulated variable in the control system (hereby referred to as the 'vaporizer' setting). The actual anesthetic concentration entering the patient's lungs is denoted as $x_{i n s p}$. We have

$$
V \dot{x}_{i n s p}=Q_{0} C_{0}-\left(Q_{0}-\Delta Q\right) x_{i n s p}-f_{R}\left(V_{T}-\Delta\right)\left(x_{i n s p}-x_{1}\right)
$$

where :

$V[1] \quad$ volume of respiratory system;

$x_{\text {insp }}[\%] \quad$ inspired drug concentration;

$x_{1}[\%] \quad$ end-tidal drug concentration;

$f_{R}[1 / \mathrm{min}] \quad$ respiratory frequency; 
$V_{T}[1] \quad$ tidal volume;

$\Delta[1] \quad$ physiological dead space

$\Delta Q[1 / \min ] \quad$ losses of breathing circuit

$Q_{0}$ and $C_{0}[\%]$ fresh gas flow and 'vaporizer setting' respectively.

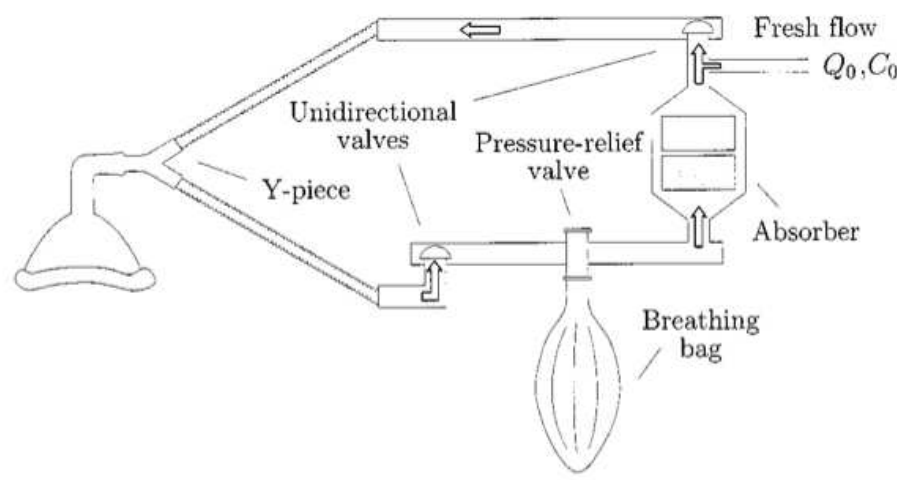

Figure 8.3: Schematic representation of breathing model as shown in [30]

The distribution of isoflurane to internal organs is modeled using the standard mammillary 5 compartment model shown in Figure 8.4. As expected the first compartment represents the lungs. The second compartment represents the heart brain and liver. The third compartment represents muscle tissue and the fourth and fifth compartments represent fatty tissues. Consider the complete pharmacokinetic models:

$$
\dot{x}_{1}=\sum_{j=2}^{5}\left(k_{j 1} x_{j} \frac{V_{j}}{V_{1}}-k_{1 j} x_{1}\right)+\frac{f_{R}\left(V_{T}-\Delta\right)}{V_{1}}\left(x_{i n s p}-x_{1}\right)
$$

For the general $j$ th compartment $(j \neq 2)$ we obtain

$$
\dot{x}_{j}=k_{1 j} x_{1} \frac{V_{1}}{V_{j}}-k_{j 1} x_{j}
$$

and for $\mathrm{j}=2$

$$
\dot{x}_{2}=k_{12} x_{1} \frac{V_{1}}{V_{2}}-k_{21} x_{2}-k_{20} x_{2}
$$




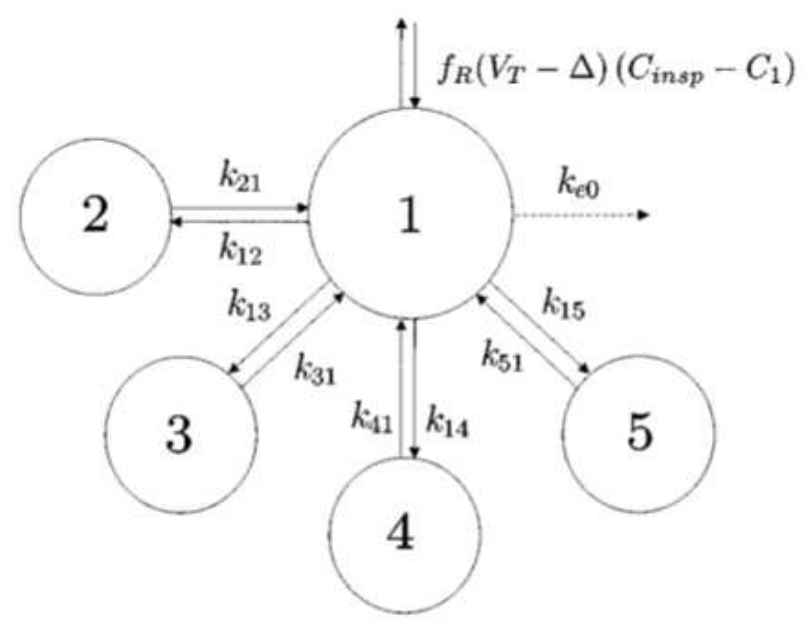

Figure 8.4: Five compartment mamilliary model

Finally, the time delay for drug flow between the central compartment and the effect compartment is given by

$$
\dot{x_{e}}=k_{e 0}\left(x_{1}-x_{e}\right)
$$

The BIS level is then related to the effect compartment concentration $x_{e}$

$$
B I S=B I S_{0}\left(1-\frac{x_{e}^{\gamma}}{x_{e}^{\gamma}+K_{50}^{\gamma}}\right)
$$

$B I S_{0}$ refers to baseline or awake state value, $B I S_{M A X}$ represents BIS with infinite isoflurane.

The overall dynamics representative of the relation between control input ('vaporizer setting') and effect compartment concentration becomes a series connection of two SISO LTI systems. Consider the first LTI system with input $u$ as vaporizer settings and output $y_{2}$ as endtidal concentration denoted by

$$
\begin{aligned}
& \dot{z}_{2}=A_{2} z_{2}+B_{2} u \\
& y_{2}=C_{2} z_{2}
\end{aligned}
$$


with the state vector

$$
z_{2}=\left[\begin{array}{c}
x_{i n s p} \\
x_{1} \\
x_{2} \\
x_{3} \\
x_{4} \\
x_{5}
\end{array}\right]
$$

Combining (8.7), (8.8) and (8.9) we have

$$
\begin{aligned}
& A_{2}=\left[\begin{array}{cccccc}
-k_{R} & k_{1 R} & 0 & 0 & 0 & 0 \\
k_{10} & -k_{1} & k_{21} \frac{V_{2}}{V_{1}} & k_{31} \frac{V_{3}}{V_{1}} & k_{41} \frac{V_{4}}{V_{1}} & k_{51} \frac{V_{5}}{V_{1}} \\
0 & k_{12} \frac{V_{1}}{V_{2}} & -k_{20}-k_{21} & 0 & 0 & 0 \\
0 & k_{13} \frac{V_{1}}{V_{3}} & 0 & -k_{31} & 0 & 0 \\
0 & k_{14} \frac{V_{1}}{V_{4}} & 0 & 0 & -k_{41} & 0 \\
0 & k_{15} \frac{V_{1}}{V_{5}} & 0 & 0 & 0 & -k_{51}
\end{array}\right] \\
& B_{2}=\left[\begin{array}{c}
Q_{0} / V \\
0 \\
0 \\
0 \\
0 \\
0
\end{array}\right] \\
& C_{2}=\left[\begin{array}{llllll}
0 & 1 & 0 & 0 & 0 & 0
\end{array}\right]
\end{aligned}
$$

where

$$
\begin{aligned}
& k_{R}=\left(Q_{0}-\Delta Q+f_{R}\left(V_{T}-\Delta\right)\right) / V \\
& k_{1 R}=f_{R}\left(V_{T}-\Delta\right) / V \\
& k_{10}=f_{R}\left(V_{T}-\Delta\right) / V_{1} \\
& k_{1}=k_{10}+k_{12}+k_{13}+k_{14}+k_{15}
\end{aligned}
$$


and the relevant volume and distribution constants given in the Figures 8.5 and 8.6.

The first LTI system is connected to the second LTI described by (8.10) and (8.11), whose input $y_{2}$ is endtidal concentration and the output $y_{1}$ the BIS. Then

$$
\begin{aligned}
& \dot{z}_{1}=A_{1} z_{1}+B_{1} y_{2} \\
& y_{1}=f\left(z_{1}\right)
\end{aligned}
$$

where $f(\cdot)$ represents the non-linear function for BIS given by $(8.11)$.

\begin{tabular}{c|c}
\hline Parameter & mean \pm SD $\left[\mathrm{min}^{-1}\right]$ \\
\hline$k_{12}$ & $1.26 \pm 0.204$ \\
\hline$k_{13}$ & $0.402 \pm 0.055$ \\
\hline$k_{14}$ & $0.243 \pm 0.072$ \\
\hline$k_{15}$ & $0.0646 \pm 0.0414$ \\
\hline$k_{20}$ & $0.0093 \pm 0.0137$ \\
\hline$k_{21}$ & $0.210 \pm 0.082$ \\
\hline$k_{31}$ & $0.0230 \pm 0.0156$ \\
\hline$k_{41}$ & $0.00304 \pm 0.00169$ \\
\hline$k_{51}$ & $0.000500 \pm 0.000119$ \\
\hline
\end{tabular}

Figure 8.5: Diffusion Constants

\begin{tabular}{c|c}
\hline Compartment & Volumes [1] (mean $\pm S D)$ \\
\hline 1 & $2.31 \pm 0.71$ \\
\hline 2 & $7.1 \pm 2.5$ \\
\hline 3 & $11.3 \pm 5.6$ \\
\hline 4 & $3.0 \pm 0.7$ \\
\hline 5 & $5.1 \pm 4.1$
\end{tabular}

Figure 8.6: Compartment Volumes

\subsection{Case 1: A Simplified model in $\mathbb{R}^{2}$}

Our objective is to demonstrate how state-space triangulation and RCP formulated on simplices can be used to realize complex control specifications. For the first example, we use a simplified 2-dimension version of the full BIS control model detailed above by ignoring some dynamics which do not affect main aspects of the problem. We also make assumptions on the model parameters and the control objectives. 
The full PK distribution model given above utilizes the 5-compartment model. Since the third, fourth and fifth compartments are not vessel-rich tissues, drug distribution within these areas is considerably slower and these compartments can be ignored. We also ignore the dynamics associated with the inspired concentration $z_{\text {insp }}$ and the closed loop breathing system, and simply assume the manipulated variable to be $I$ : anesthetic infusion rate into the central compartment. We have:

$$
\left[\begin{array}{c}
\dot{x_{1}} \\
\dot{x_{2}}
\end{array}\right]=\left[\begin{array}{cc}
-k_{1} & k_{21} \frac{V_{2}}{V_{1}} \\
k_{12} \frac{V_{1}}{V_{2}} & -k_{20}-k_{21}
\end{array}\right]\left[\begin{array}{l}
x_{1} \\
x_{2}
\end{array}\right]+\left[\begin{array}{c}
1 \\
0
\end{array}\right] I
$$

$x_{1}$ is the drug concentration in the first compartment

$x_{2}$ is the drug concentration in the second compartment

$k_{1}=k_{10}+k_{12}$

In [31] , typical ranges for parameters $f_{R}, V_{T}, \Delta, Q_{0}$, and $\Delta Q$ are given. Using $f_{R}=4[1 / \mathrm{min}], V_{T}=0.3[1], \Delta=0.1268$ and equation (8.18) we calculate $k_{10}=0.3$. We assume that the compartmental volume ratio $\frac{V_{1}}{V_{2}}=1$, and ignore drug metabolization in the liver so that $k_{20}=0$. (The choice of parameters and specific assumptions allow for interesting complex specifications for this 2-dimensional system). Obtaining $k_{12}$ and $k_{21}$ from Figures 8.5 and 8.6 we have:

$$
\left[\begin{array}{c}
\dot{x_{1}} \\
\dot{x_{2}}
\end{array}\right]=\left[\begin{array}{cc}
-1.56 & 0.210 \\
1.26 & -0.210
\end{array}\right]\left[\begin{array}{l}
x_{1} \\
x_{2}
\end{array}\right]+\left[\begin{array}{l}
1 \\
0
\end{array}\right] I
$$

Also the set of possible equilibria associated with the dynamics is:

$$
\mathcal{O}=\left\{x \mid 1.26 x_{1}-0.210 x_{2}=0\right\}
$$

In the original model the effect compartment models the transport time of the drug to the effect site. We ignore the transport time so the BIS effect is based on the first compartment concentration $x_{1}$ instead of effect compartment concentration $x_{e}$ and we have

$$
B I S=B I S_{0}\left(1-\frac{x_{1}^{\gamma}}{x_{1}^{\gamma}+K_{50}^{\gamma}}\right)
$$


Using data from [30] we use $K_{50}=0.7495$ and $\gamma=2$. For measuring anesthesia depth we have a maximum BIS value $B I S_{0}=100$, with minimum BIS assumed to be 0 .

\subsubsection{Complex Control Specifications}

The primary control objective of anesthesia induction is to bring the patient from fully conscious (BIS levels around 100), to Stage 3 deep hypnosis (BIS levels below 40), so that surgery can be performed. (After commencement of surgery anesthesia depth is maintained at a relatively constant level until surgery is complete). Consider a summary of the transient stages of anesthesia induction and the corresponding BIS values for each stage as given in [33].

- Stage 1 Stage 1 anesthesia is the period between the initial administration of induction medications and the loss of consciousness. During this stage drowsiness and dizziness are accompanied by auditory and visual hallucinations until loss of consciousness. A BIS level of $75.3 \pm 2.85$ is associated with onset of this stage.

- Stage 2 Stage 2 anesthesia also known as the excitement stage is the period following the loss of consciousness and marked by excited and delirious activity. The BIS level associated with this stage is $93.1 \pm 3.1$, thus indicating that there is an increase in BIS levels soon after Stage 1. This increase is due to excessive muscle activity and excitement. We note that the pharmacodynamic model used in our control design does not take into consideration such effects and increasing the concentration of drug in the body is only associated with a decrease in BIS levels. In other words, the transitory spike in BIS associated with Stage 2 anesthesia will not be visible using the current model.

- Stage 3 This stage resembles natural sleep whereby surgery can begin. Stage 3-plane I, marked by slowing and stopping of eye movements, corresponds to a BIS level of $41.2 \pm 2.32$. Stage 3 -plane II is marked by loss of corneal reflexes and 
associated with a BIS level of $30.8 \pm 1.95$. Stage 3-plane III, marked by intercostal paralysis is associated with a BIS level of $24.5 \pm 1.08$. We assume that surgery can take place only after Stage 3 -plane III is reached.

The BIS variation during these stages of anesthesia as studied in [33] is pictured in Figure 8.7. As mentioned, the temporary increase in BIS values after Stage 1 associated with the onset of Stage 2, is not possible under the current model. Nonetheless, since the objective of the example is to illustrate how complex specifications arise and are implemented, we assume that the required BIS specification is as shown as in Figure 8.7. Since BIS levels are related to central compartment concentration, $x_{1}$ is required to evolve in such a manner that BIS values obey the specified pattern.

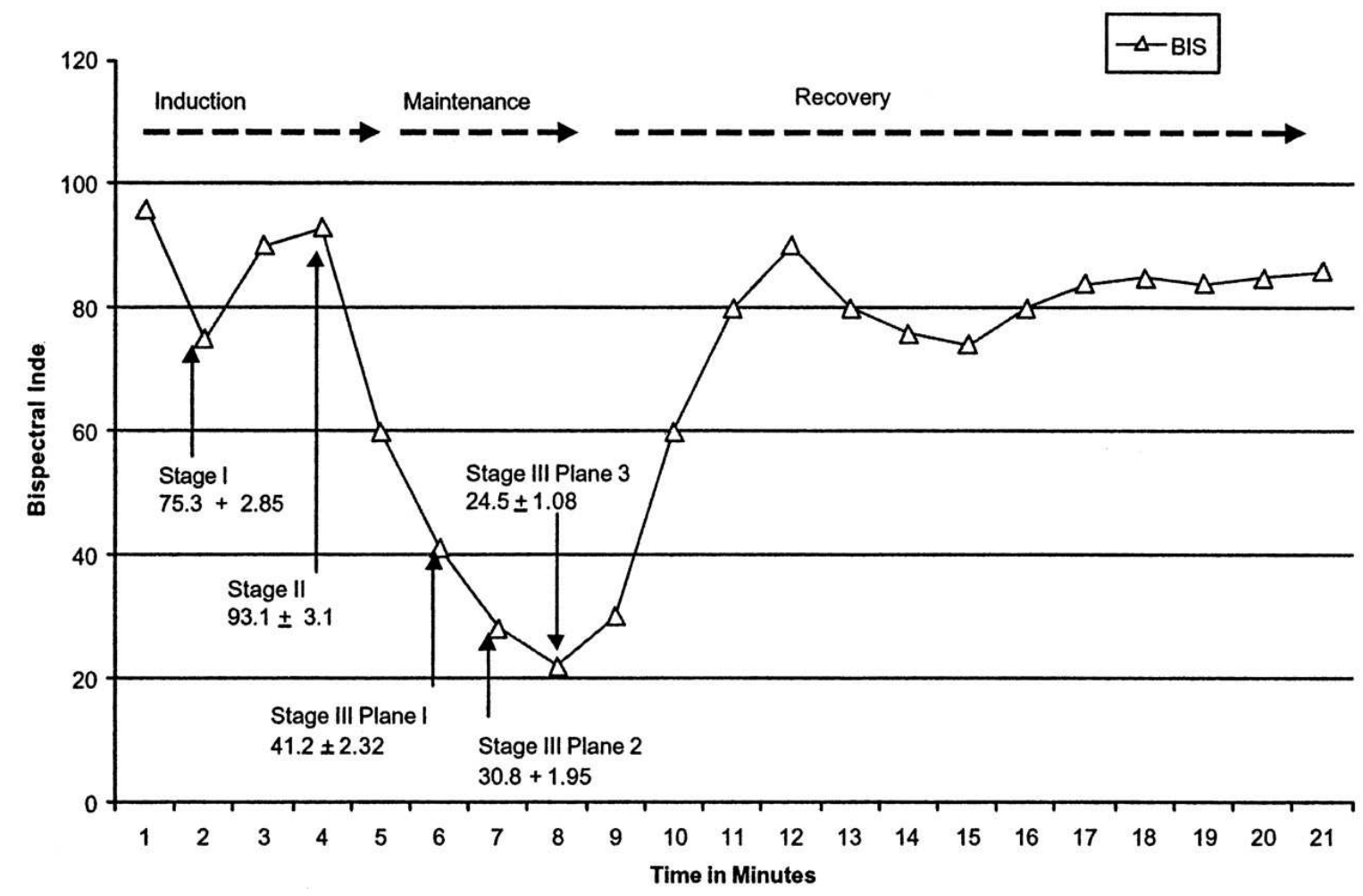

Figure 8.7: BIS variation under various stages of anesthesia as reported in [33]

In addition to requirements on the manner of variation of the BIS value, it is reasonable to assume other requirements may exist which increase the overall complexity of the control specification. We now propose some specifications for the evolution of 
drug concentration in the peripheral compartment. These requirements are not standard and their purpose is to illustrate how complex specification may arise and be realized. Nonetheless sufficient engineering judgment and intuition are used to provide realistic and acceptable scenarios.

(1) From (8.23), it can be seen that as the amount of drug in the central compartment, $x_{1}$ increases, the rate of increase of the drug in the peripheral compartment, $x_{2}$ increases. From the point of initial drug administration to the onset of Stage 3 anesthesia, it may be required to limit the of increase of $x_{2}$. Such a requirement may be necessary to gradually introduce the drug to vital tissues and organs during the induction of unconsciousness to verify whether the patient is sensitive to the inhaled drug and thereby avoid dangerous scenarios. For example the supervising anesthesiologist may want to ensure that the patient's cardiac muscles (the heart is modeled in compartment 2) respond well to isoflurane and hence only allow compartment 2 concentrations to gradually increase as hypnosis deepens, while carefully monitoring cardiac stability signs such as heart rate and blood pressure. The limitations on the rate of increase of $x_{2}$ can be done as follows:

(i) During the transition from fully awake to Stage 1, as well as at the actual onset of Stage 1, $x_{2}$ should be no greater than $0.1 \%$

(ii) During the transition from Stage 1 to Stage 2, as well as at the actual onset of Stage $2, x_{2}$ should be no greater than $0.35 \%$.

(iii) During the transition from Stage 2 to Stage 3 plane I, as well as at the actual onset of Stage 3 plane I, $x_{2}$ should be no greater than $0.6 \%$.

(2) During surgery (assumed to be performed at Stage 3 -plane III) the amount of anesthetic drug circulating in vessel-rich tissues may be required to be in the range $0.7-1 \%$. A requirement to keep the drug levels at a minimum value $(0.7 \%)$ during the surgical stage may exist so as to ensure patients do not drift out of 
this stage without using further large infusions of the drug. The upper limit of $1 \%$ may be enforced to maintain patient safety and reduce the risk of side-effects or complications associated with large drug concentrations in vital organs. The increase in drug concentration to the target zone could be done in the following manner:

(i) At the onset of Stage 3 Plane I, $x_{2}$ should be no less than $0.35 \%$.

(ii) During the transition from Stage 3 Plane I to Plane II, $x_{2}$ should greater than $0.35 \%$ but no greater than $0.7 \%$. At the actual onset of Stage 3 Plane II, $x_{2}$ should be no less than $0.4 \%$ but no greater than $0.7 \%$

(iii) During the transition from Stage 3 plane II to plane III, $x_{2}$ should be greater than $0.4 \%$ but no greater than $1 \%$.

(iv) At the actual onset of Stage 3 Plane III, $x_{2}$ should be in the target zone, i.e. no less than $0.7 \%$ but no greater than $1 \%$.

\subsubsection{Control Implementation}

The control strategy to realize the complex specifications of the anesthesia induction process is modeled as a discrete automaton as shown in Figure 8.8. Each discrete event is representative of the onset of a specific stage of anesthesia in the controlled induction. Each mode is representative of the transition between individual stages of anesthesia. Consider the modes and events illustrated in the automaton in Figure 8.8.

We determine the specifications on each state for every mode and event of the automaton. Since stages of anesthesia are defined by BIS values, we obtain the corresponding ranges and values of $x_{1}$ associated with each mode and event by observing Figure 8.9, a plot of BIS versus $x_{1}$. Combining with the constraints on $x_{2}$, we now separate the statespace into suitable areas which satisfy the constraints. This is shown in Figure 8.10. Note that we assume areas in the state-space which are designated to different modes 


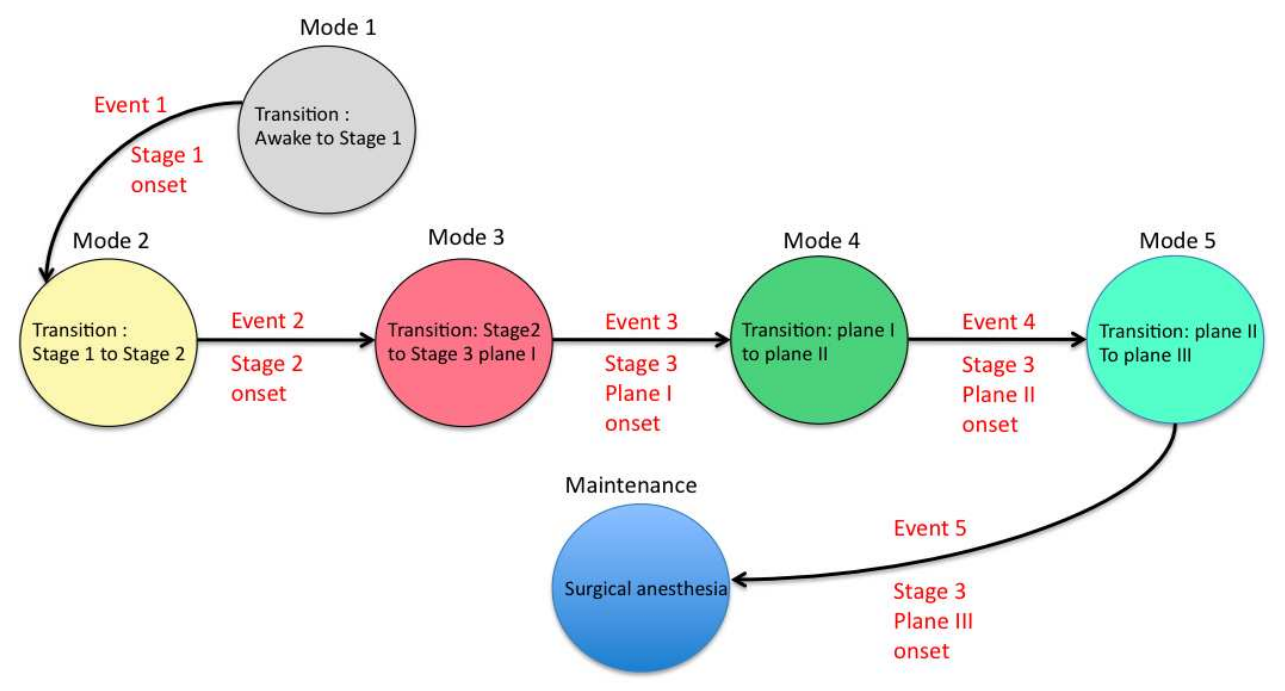

Figure 8.8: Discrete Automaton representing control specification: Case 1

must be unique with no area shared between modes.

Mode 1: Transition from Full consciousness to Stage 1:

$$
\begin{aligned}
& 0 \leq x_{1}<0.5 \\
& 0 \leq x_{2} \leq 0.1
\end{aligned}
$$

Event 1: Onset of Stage 1:

$$
\begin{aligned}
x_{1} & =0.5 \\
0 \leq x_{2} & \leq 0.1
\end{aligned}
$$

Mode 2: Transition from Stage 1 to Stage 2:

$$
\begin{array}{r}
0.5<x_{1} \leq 0.55 \\
0 \leq x_{2} \leq 0.35
\end{array}
$$

and

$$
\begin{gathered}
0.3<x_{1} \leq 0.5 \\
0.1 \leq x_{2} \leq 0.35
\end{gathered}
$$


Event 2: Onset of Stage 2:

$$
\begin{array}{r}
x_{1}=0.3 \\
0.1 \leq x_{2} \leq 0.35
\end{array}
$$

Mode 3: Transition from Stage 2 to Stage 3 Plane I:

$$
\begin{aligned}
& 0.2 \leq x_{1}<0.3 \\
& 0.1 \leq x_{2} \leq 0.6
\end{aligned}
$$

and

$$
\begin{array}{r}
0.2 \leq x_{1}<1 \\
0.35 \leq x_{2} \leq 0.6
\end{array}
$$

Event 3: Onset of Stage 3 Plane I:

$$
\begin{array}{r}
x_{1}=1 \\
0.35 \leq x_{2} \leq 0.6
\end{array}
$$

Mode 4:Transition from Stage 3 Plane I to Stage 3 Plane II:

$$
\begin{array}{r}
1<x_{1}<1.3 \\
0.35<x_{2} \leq 0.7
\end{array}
$$

Event 4: Onset of Stage 3 Plane II:

$$
\begin{aligned}
x_{1} & =1.3 \\
0.4 \leq x_{2} & \leq 0.7
\end{aligned}
$$

Mode 5: Transition from Stage 3 Plane II to Stage 3 Plane III :

$$
\begin{gathered}
1.3<x_{1}<1.5 \\
0.4<x_{2} \leq 1
\end{gathered}
$$


Event 5: Onset of Stage 3 Plane III:

$$
\begin{array}{r}
x_{1}=1.5 \\
0.7 \leq x_{2} \leq 1
\end{array}
$$

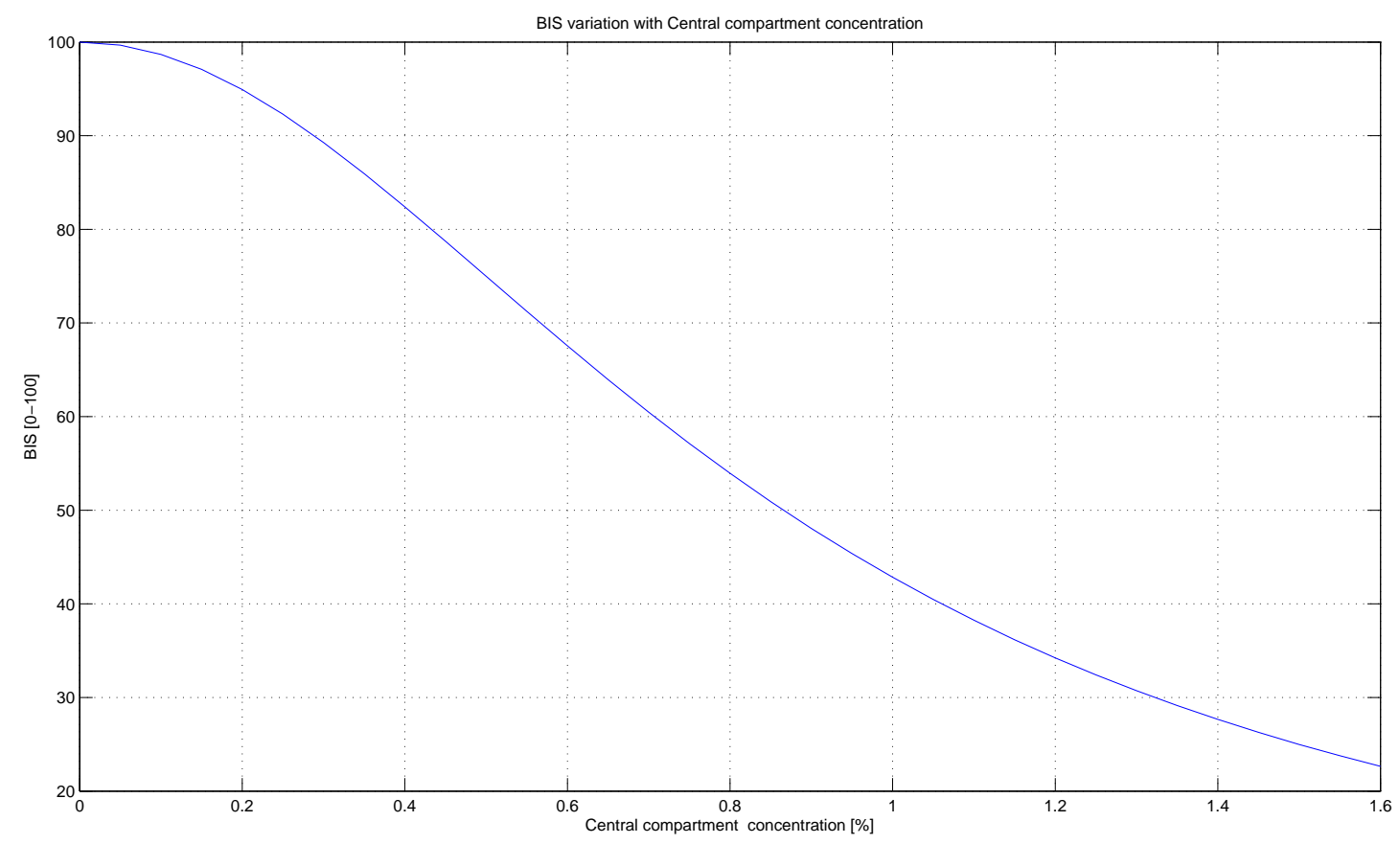

Figure 8.9: BIS variation with central compartment concentration

We now triangulate the state-space within these selected areas into a sequence of simplices through which the state can evolve in order to satisfy the rules of the automaton and realize the complex control specification. The triangulation is performed in such a manner so as to ensure existence of geometric sufficient conditions developed in the theory for solvability of RCP on each simplex. Table 8.1 gives the vertex data set for the 13 simplices of the proposed triangulation. Consider $\mathcal{S}_{2}-\mathcal{S}_{13}$. The set $\mathcal{O}$ is defined in (8.25) and we have $\mathcal{G}_{k}=\mathcal{S}_{k} \cap \mathcal{O}=\emptyset$, for $k=2, \ldots, 13$. Also, the invariance conditions for each $\mathcal{S}_{k}$ can be shown to be solvable, so by Theorem $4.1 \mathrm{RCP}$ is solvable for $\mathcal{S}_{2}-\mathcal{S}_{13}$. Now consider $\mathcal{S}_{1}$. We have $\mathcal{G}_{1}=\mathcal{S}_{1} \cap \mathcal{O}=v_{0}$. However it is easily shown $\mathcal{B} \cap \operatorname{cone}\left(\mathcal{S}_{1}\right) \neq \mathbf{0}$ 


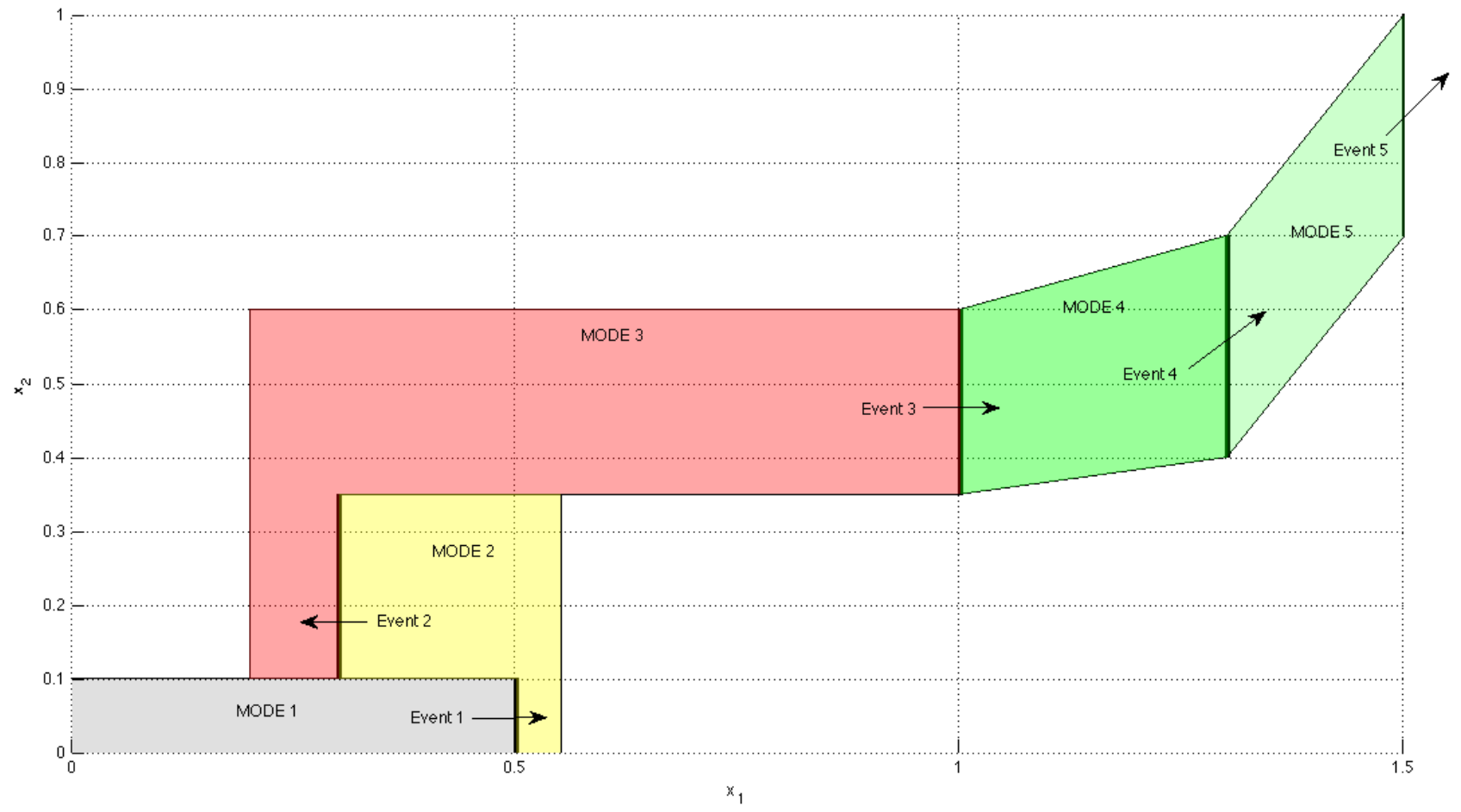

Figure 8.10: State-space areas satisfying constraints for modes/events of control specification (Case 1) 
and the invariance conditions are solvable. Therefore by Theorem 4.2 RCP is solvable for $\mathcal{S}_{1}$.

\begin{tabular}{|c|c|c|c|}
\hline \multicolumn{4}{|c|}{ Simplex Data } \\
\hline $\mathcal{S}_{k}$ & $v_{0}$ & $v_{1}$ & $v_{2}$ \\
\hline $\mathcal{S}_{1}$ & $(0,0)$ & $(0.5,0)$ & $(0.5,0.1)$ \\
\hline $\mathcal{S}_{2}$ & $(0.5,0)$ & $(0.5,0.1)$ & $(0.53,0.06)$ \\
\hline $\mathcal{S}_{3}$ & $(0.53,0.06)$ & $(0.55,0.1)$ & $(0.5,1)$ \\
\hline $\mathcal{S}_{4}$ & $(0.55,0.1)$ & $(0.5,0.1)$ & $(0.3,0.35)$ \\
\hline $\mathcal{S}_{5}$ & $(0.5,0.1)$ & $(0.30,0.15)$ & $(0.30,0.35)$ \\
\hline $\mathcal{S}_{6}$ & $(0.30,0.15)$ & $(0.30,0.35)$ & $(0.20,0.35)$ \\
\hline $\mathcal{S}_{7}$ & $(0.20,0.35)$ & $(0.3,0.35)$ & $(0.3,0.45)$ \\
\hline $\mathcal{S}_{8}$ & $(0.3,0.35)$ & $(1,0.35)$ & $(0.3,0.45)$ \\
\hline $\mathcal{S}_{9}$ & $(0.3,0.45)$ & $(1,0.35)$ & $(1,0.55)$ \\
\hline $\mathcal{S}_{10}$ & $(1,0.35)$ & $(1.3,0.4)$ & $(1,0.55)$ \\
\hline $\mathcal{S}_{11}$ & $(1,0.55)$ & $(1.3,0.7)$ & $(1.3,0.4)$ \\
\hline $\mathcal{S}_{12}$ & $(1.3,0.4)$ & $(1.5,0.7)$ & $((1.3,0.7)$ \\
\hline $\mathcal{S}_{13}$ & $(1.3,0.7)$ & $(1.5,0.7)$ & $(1.5,1)$ \\
\hline
\end{tabular}

Table 8.1: Simplex data (Case 1)

For each simplex we choose control inputs at the vertices to satisfy the relevant invariance conditions and calculate the corresponding affine feedback law which solves 
RCP. The resulting piecewise affine feedback law is as follows:

$$
\begin{aligned}
& u=\left[\begin{array}{ll}
1.4 & 31
\end{array}\right] x+0.2, \quad x \in \mathcal{S}_{1} \\
& u=\left[\begin{array}{ll}
-40.4 & 22.2
\end{array}\right] x+20.98, \quad x \in \mathcal{S}_{2} \\
& u=\left[\begin{array}{ll}
-24 & -13
\end{array}\right] x+14.5, \quad x \in \mathcal{S}_{3} \\
& u=\left[\begin{array}{ll}
0 & 0
\end{array}\right] x, \quad x \in \mathcal{S}_{4} \\
& u=\left[\begin{array}{ll}
0 & 0
\end{array}\right] x, \quad x \in \mathcal{S}_{5} \\
& u=\left[\begin{array}{ll}
-9 & -1.5
\end{array}\right] x+3.325, \quad x \in \mathcal{S}_{6} \\
& u=\left[\begin{array}{ll}
-3 & 15
\end{array}\right] x-3.85, \quad x \in \mathcal{S}_{7} \\
& u=2, \quad x \in \mathcal{S}_{8} \\
& u=\left[\begin{array}{ll}
0.7143 & 45
\end{array}\right] x-15.4634, \quad x \in \mathcal{S}_{9} \\
& u=\left[\begin{array}{ll}
-11.667 & 10
\end{array}\right] x+11.1667, \quad x \in \mathcal{S}_{10} \\
& u=\left[\begin{array}{ll}
-5 & 16.667
\end{array}\right] x-0.1667, \quad x \in \mathcal{S}_{11} \\
& u=\left[\begin{array}{ll}
-5 & 1.667
\end{array}\right] x+8.333, \quad x \in \mathcal{S}_{12} \\
& u=\left[\begin{array}{ll}
-20 & 13.33
\end{array}\right] x+20.667, \quad x \in \mathcal{S}_{13}
\end{aligned}
$$

The control using the piecewise affine feedback law is simulated for a patient initially at full consciousness. The state trajectory evolves from the origin (corresponding to no drug present in the body) through the required path of simplices as shown in Figure 8.11. Figure 8.12 shows that the BIS level evolves according to the specification outlined, moving smoothly from 100 to slightly below 75 , increasing back up to around 90 and then finally decreasing to around 20, which corresponds to Stage 3-Plane III anesthesia depth. Moreover, it is clear that throughout the anesthesia induction, the control strategy simultaneously ensures the precise transient specification for the drug concentration in the 
peripheral compartment is obeyed. This is shown in Figure 8.14. The use of triangulation and piecewise affine feedback is therefore successful in implementing the set of complex specifications.

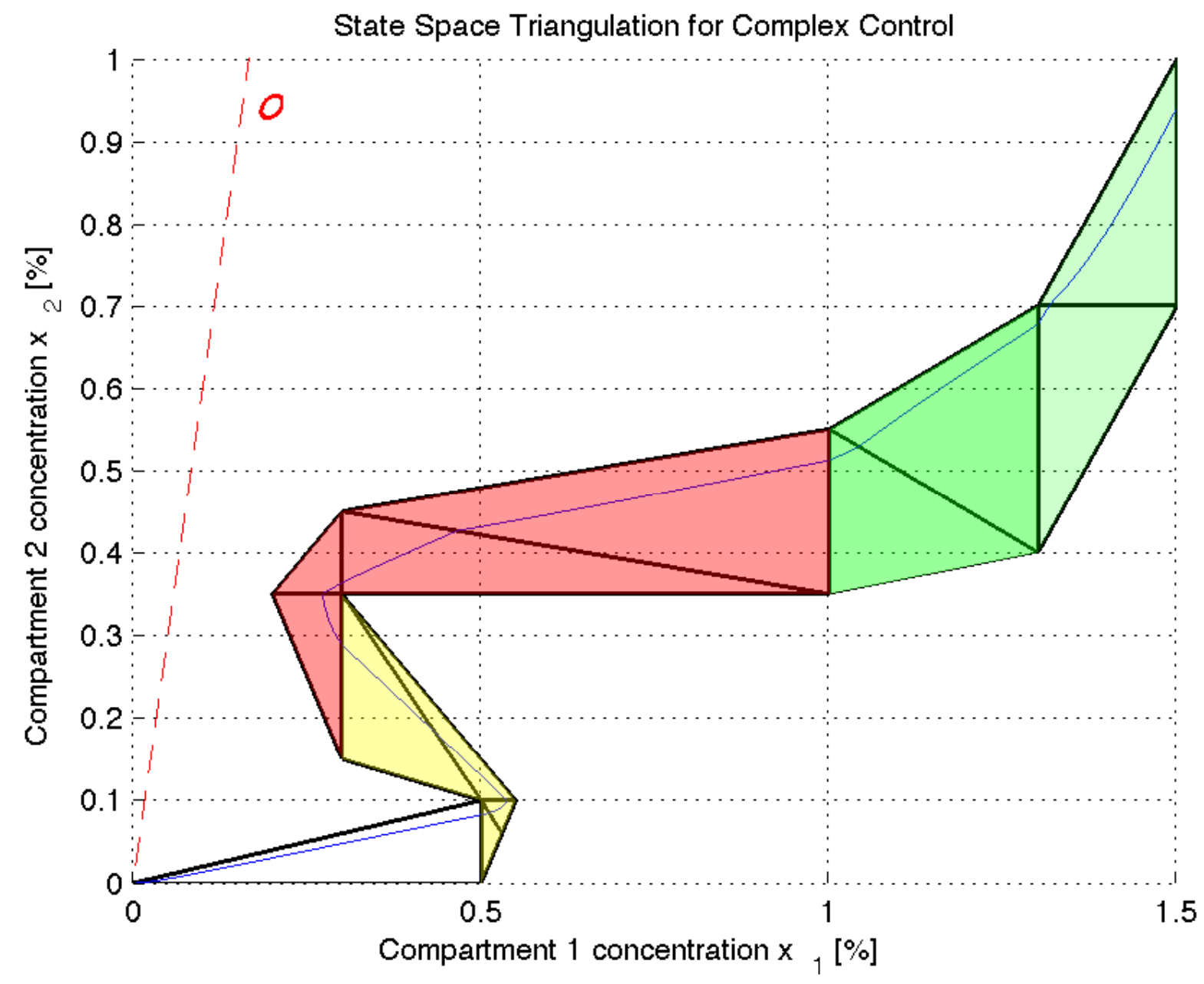

Figure 8.11: Triangulated state-space showing state trajectory under piecewise affine controller: Case 1

\subsection{Case 2: Simplified model in $\mathbb{R}^{3}$}

We now use another simplified model to demonstrate in three-dimensions, the idea of complex specifications and the use of triangulation and RCP formulated on simplices in 


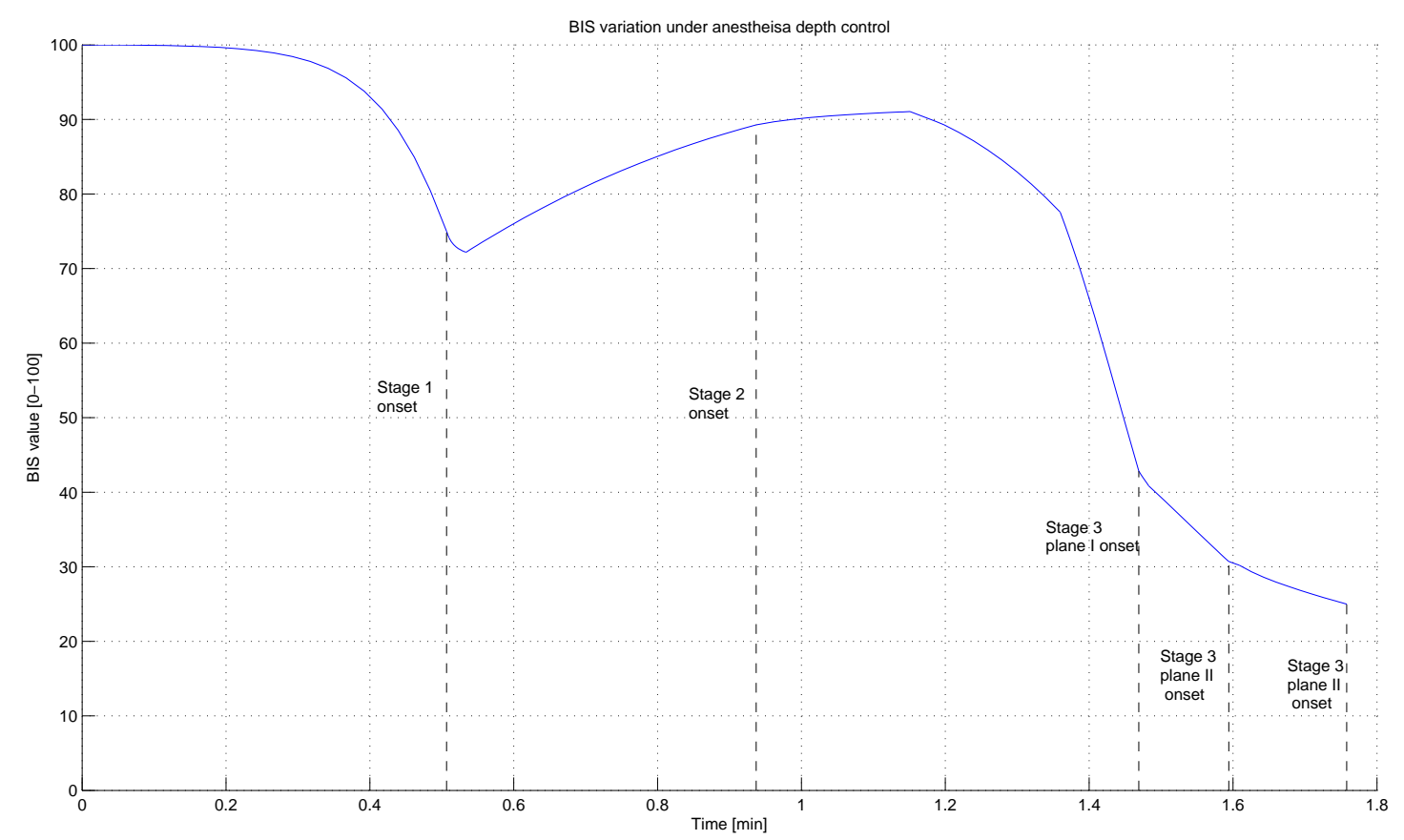

Figure 8.12: BIS variation under piecewise affine controller: Case 1

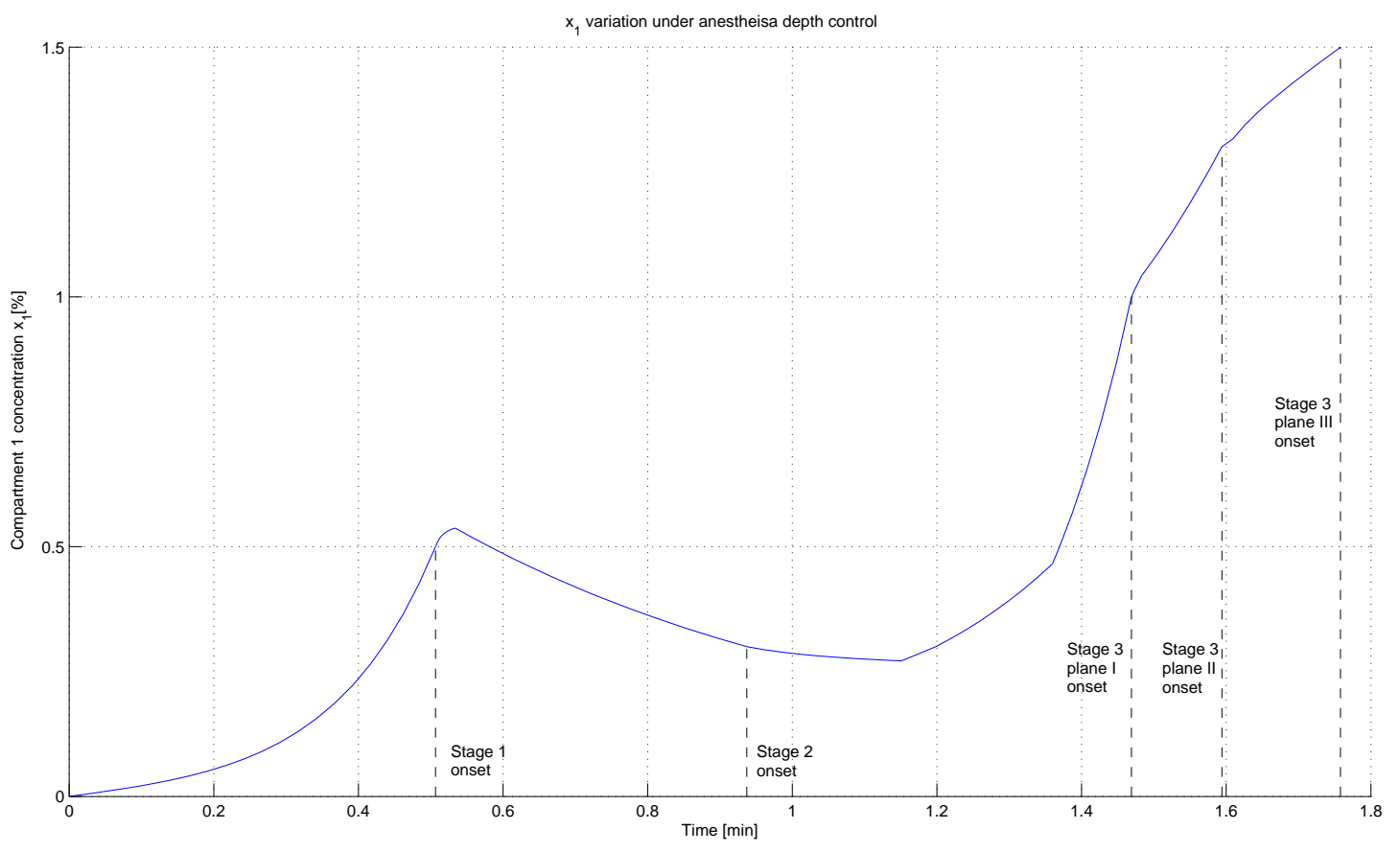

Figure 8.13: $x_{1}$ variation under piecewise affine controller: Case 1 


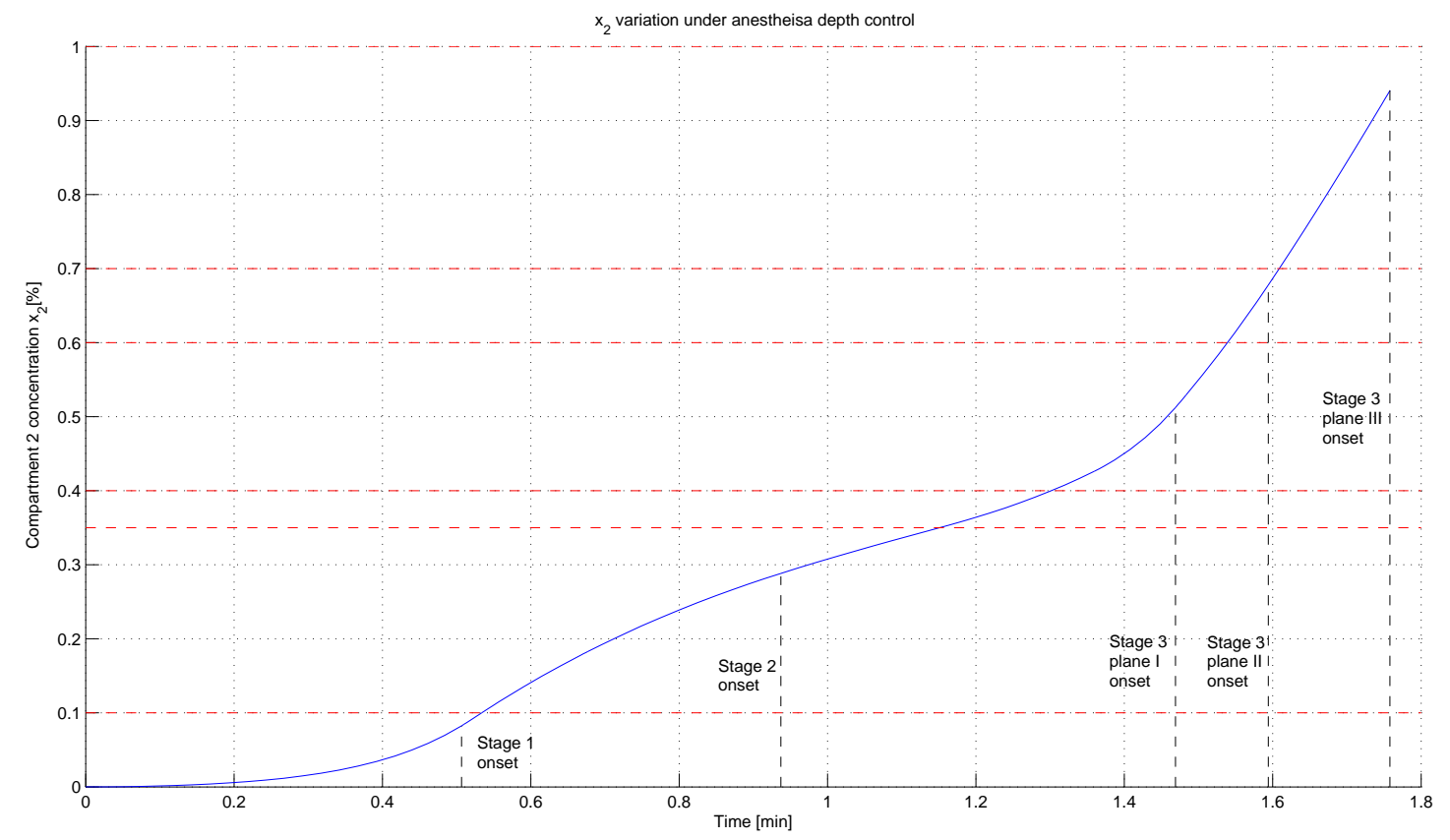

Figure 8.14: $x_{2}$ variation under piecewise affine controller: Case 1

their implementation.

The third, fourth and fifth compartments are not vessel-rich tissues and since drug distribution is considerably slower in these areas we ignore these peripheral compartments. Unlike in Case 1, the dynamics contributed by the breathing machine are retained and the control input is the vaporizer setting, $C_{0}$. We have

$$
\left[\begin{array}{c}
\dot{x_{1}} \\
\dot{x_{2}} \\
\dot{x_{3}}
\end{array}\right]=\left[\begin{array}{ccc}
-k_{R} & k_{1 R} & 0 \\
k_{10} & -k_{1} & k_{21} \frac{V_{2}}{V_{1}} \\
0 & k_{12} \frac{V_{1}}{V_{2}} & -k_{20}-k_{21}
\end{array}\right]\left[\begin{array}{c}
x_{1} \\
x_{2} \\
x_{3}
\end{array}\right]+\left[\begin{array}{c}
Q_{0} / V \\
0 \\
0
\end{array}\right] C_{0}
$$

where

$x_{1}$ is the inspired drug concentration

$x_{2}$ is the drug concentration in the first compartment

$x_{3}$ is the drug concentration in the second compartment

$k_{1}=k_{10}+k_{12}$

To maintain accuracy, we use the parameters for a typical anesthesia procedure as 
given in [30] : $f_{R}=10[1 / \mathrm{min}], V_{T}=0.6[1], \Delta=0.15, Q_{0}=1[1 / \mathrm{min}], \Delta Q=0.2[1 / \mathrm{min}]$. Furthermore, using data in Figures 8.5 and 8.6 we have

$$
\left[\begin{array}{c}
\dot{x_{1}} \\
\dot{x_{2}} \\
\dot{x_{3}}
\end{array}\right]=\left[\begin{array}{ccc}
-1.06 & 0.9 & 0 \\
1.9481 & -3.2081 & 0.6455 \\
0 & 0.4099 & -0.2193
\end{array}\right]\left[\begin{array}{c}
x_{1} \\
x_{2} \\
x_{3}
\end{array}\right]+\left[\begin{array}{c}
0.2 \\
0 \\
0
\end{array}\right] u
$$

Also the set of possible equilibria associated with the dynamics is:

$$
\mathcal{O}=\left\{x \mid 1.9481 x_{1}-3.2081 x_{2}+0.6455 x_{3}=0,0.4099 x_{2}-0.2193 x_{3}=0\right\}
$$

For safety we assume the maximum value of our control input is $15 \%$.

As in Case I, we ignore the transport time. Hence the BIS effect is based on the first compartment concentration $x_{2}$ instead of effect compartment concentration. The non-linear function relating BIS to $x_{2}$ becomes

$$
B I S=B I S_{0}\left(1-\frac{x_{2}^{\gamma}}{x_{2}^{\gamma}+K_{50}^{\gamma}}\right)
$$

As in Case 1 we use $K_{50}=0.7495$ and $\gamma=2$. For measuring anesthesia depth we have a maximum BIS values $B I S_{0}=100$, with minimum BIS assumed to be 0 .

\subsubsection{Complex Control Specifications}

The primary control objective is to bring the patient from fully conscious (BIS levels around 100), to Stage 3 deep hypnosis (BIS levels below 40), so that surgery can be performed. The corresponding BIS values for each stage was reviewed earlier. As discussed also, the onset of Stage 2 anesthesia cannot be recognized using our model, and hence it will be assumed as part of the transition from Stage 1 to Stage 3. Since BIS levels are related to central compartment concentration, $x_{2}$ is required to increase to appropriate levels so as to smoothly bring BIS values from 100 to $24.5 \pm 1.08$. We now propose some specifications for the evolution of the inspired drug concentration and concentration in 
the peripheral compartment during induction. As in the previous example, these requirements are not standard and their purpose is to illustrate how complex specification may arise and be realized.

The 'vaporizer setting' $C_{0}$, is the manipulated variable in the closed loop system and directly controls the inspired drug concentration $x_{1}$, that is, the drug concentration entering the lungs. The inspired concentration is coupled to the dynamics of $x_{2}$ (which determines anesthesia depth), and also the dynamics of the peripheral compartment $x_{3}$. It is therefore reasonable to assume that some requirements on the inspired concentration may exist to optimize the induction process.

(1) The inspired concentration $x_{1}$ should be increased steadily from between $0 \%$ and $2 \%$ before the onset of Stage 1 anesthesia and at the onset of Stage 1 anesthesia, should be in the range $1 \%$ and $2 \%$. The requirement to increase $x_{1}$ to a minimum value of at least $1 \%$ at the onset of Stage 1, may be to ensure inspired concentrations are high enough to continue the fast transition to Stage 3 without requiring too sudden and possibly dangerous increases in vaporizer setting to keep $\dot{x_{2}}$ positive. The drug concentration entering the lungs is limited to $2 \%$ so that any sensitivities and potentially fatal reactions associated with introducing the anesthetic into the respiratory system can be detected before Stage 1 unconsciousness is induced and inspired concentrations are increased further.

(2) The inspired concentration $x_{1}$ should then be increased gradually during the transition from Stage 1 to Stage 3 plane III, so that $x_{1}$ is in the target range $2.6-3.0 \%$ by the onset of Stage 3 plane III. Inspired concentrations may be required to be in this target zone at the onset of Stage 3 plane III so that it is high enough during the surgery and it may be easier to ensure patients do not drift out of this stage without using further large infusions of the drug. The upper limit of 3.0\% may exist so that inspired concentration is kept within physiologically safe limits. Consider a possible evolution requirement on $x_{1}$ during the transition from Stage 1 to Stage 
3 plane III:

(i) During the transition from Stage 1 to Stage 3 plane I, $x_{1}$ should be greater than $1 \%$ and no greater than $2.25 \%$. At the onset of Stage 3 plane I, $x_{1}$ should be in the range $1.75 \%$ to $2.25 \%$.

(ii) During the transition from Stage 3 plane I to Stage 3 plane II, $x_{1}$ should be greater than $1.75 \%$ and no greater than $2.6 \%$. At the onset of Stage 3 plane I, $x_{1}$ should be in the range $2.25 \%$ to $2.6 \%$.

(iii) During the transition from Stage 3 plane II to Stage 3 plane III, $x_{1}$ should be greater than $2.25 \%$ and no greater than $3.0 \%$. At the onset of Stage 3 plane III, $x_{1}$ should be in the target zone $2.6 \%$ to $3.0 \%$.

Since the second compartment is connected to the first, as $x_{2}$ increases we expect an increase in $x_{3}$. The second compartment is representative of vessel rich tissues and vital organs such as heart, brain and liver and it is reasonable to assume that in some patients (for example patients with liver conditions or other problems where tissue may be sensitive to chemicals) it may be beneficial to minimize the amount of drug circulating in these organs. Thus we place the following restrictions on the evolution of $x_{3}$.

(1) At the onset of Stage 1, $x_{3}$ should be limited to a maximum value of $40 \%$ of $x_{2}$. This allows any sensitivities and potentially fatal reactions associated with introducing the anesthetic into vital organs (e.g. heart and central nervous system) to be detected before deep levels of unconsciousness are induced.

(2) At the onset of Stage 3 plane I, $x_{3}$ should be limited to a maximum value of $50 \%$ of $x_{2}$.

(3) At the onset of Stage 3 plane II, $x_{3}$ should be limited to a maximum value of $60 \%$ of $x_{2}$. 
(4) At the onset of Stage 3 plane III, $x_{3}$ should be limited to a maximum value of $70 \%$ of $x_{2}$.

\subsubsection{Control Implementation}

The discrete automaton representative of the proposed control strategy is outlined below in Figure 8.15. Note the 4 main modes.

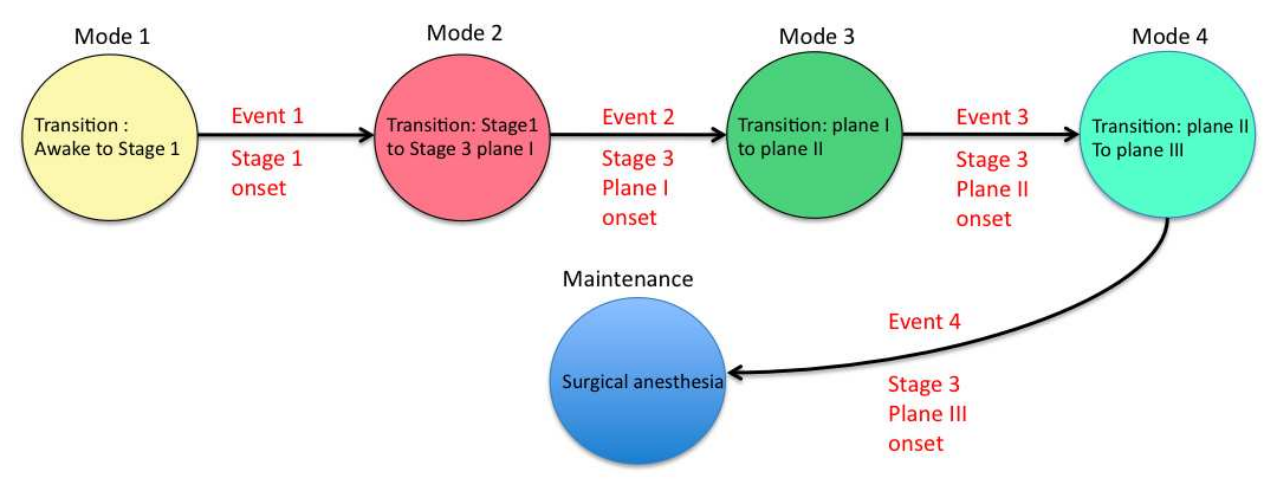

Figure 8.15: Discrete Automaton representing control specification: Case 2

From Figure 8.9 we observe the variation of BIS with central compartment concentration $x_{2}$ and combining with constraints on $x_{1}$ and $x_{3}$, we identify general state-space regions which satisfy the constraints of the modes and events of the automaton. This is shown in Figure 8.16.

Mode 1: Transition from Full consciousness to Stage 1:

$$
\begin{array}{r}
0 \leq x_{1} \leq 2 \\
0 \leq x_{2}<0.5 \\
x_{3} \leq 0.2
\end{array}
$$


Event 1: Onset of Stage 1:

$$
\begin{array}{r}
1 \leq x_{1} \leq 2 \\
x_{2}=0.5 \\
x_{3} \leq 0.2
\end{array}
$$

Mode 2: Transition from Stage 1 to Stage 3 plane I:

$$
\begin{array}{r}
1 \leq x_{1} \leq 2.25 \\
0.5<x_{2}<1 \\
x_{3} \leq 0.5
\end{array}
$$

Event 2: Onset of Stage 3 plane I:

$$
\begin{array}{r}
1.75 \leq x_{1} \leq 2.25 \\
x_{2}=1 \\
x_{3} \leq 0.5
\end{array}
$$

Mode 3: Transition from Stage 3 plane I to II:

$$
\begin{array}{r}
1.75 \leq x_{1} \leq 2.6 \\
1<x_{2}<1.3 \\
x_{3} \leq 0.78
\end{array}
$$

Event 3: Onset of Stage 3 plane II:

$$
\begin{array}{r}
2.25 \leq x_{1} \leq 2.6 \\
x_{2}=1.3 \\
x_{3} \leq 0.78
\end{array}
$$


Mode 4: Transition from Stage 3 plane II to III:

$$
\begin{array}{r}
2.25 \leq x_{1} \leq 3.0 \\
1.3<x_{2}<1.5 \\
x_{3} \leq 1.05
\end{array}
$$

Event 4: Onset of Stage 3 plane III:

$$
\begin{array}{r}
2.6 \leq x_{1} \leq 3.0 \\
x_{2}=1.5 \\
x_{3} \leq 1.05
\end{array}
$$

We now triangulate the state-space within these selected areas into a sequence of simplices through which the state can evolve in order to satisfy the rules of the automaton and realize the complex control specification. As in Case 1, the triangulation is performed in such a manner so as to ensure existence of geometric sufficient conditions on each simplex for solvability of RCP. Table 8.2 gives the vertex data set for the 11 simplices of the proposed triangulation. Consider $\mathcal{S}_{2}-\mathcal{S}_{11}$. The set $\mathcal{O}$ is defined in (8.29) and we have $\mathcal{G}_{k}=\mathcal{S}_{k} \cap \mathcal{O}=\emptyset$, for $k=2, \ldots, 11$. Also, the invariance conditions for each $\mathcal{S}_{k}$ can be shown to be solvable, so by Theorem $4.1 \mathrm{RCP}$ is solvable for $\mathcal{S}_{2}-\mathcal{S}_{11}$. Now consider $\mathcal{S}_{1}$. We have $\mathcal{G}_{1}=\mathcal{S}_{1} \cap \mathcal{O}=v_{0}$. However it is easily shown $\mathcal{B} \cap \operatorname{cone}\left(\mathcal{S}_{1}\right) \neq \mathbf{0}$ and the invariance conditions are solvable. Therefore by Theorem $4.2 \mathrm{RCP}$ is solvable for $\mathcal{S}_{1}$.

For each simplex we choose control inputs at the vertices to satisfy the relevant invariance conditions and calculate the corresponding affine feedback law which solves 


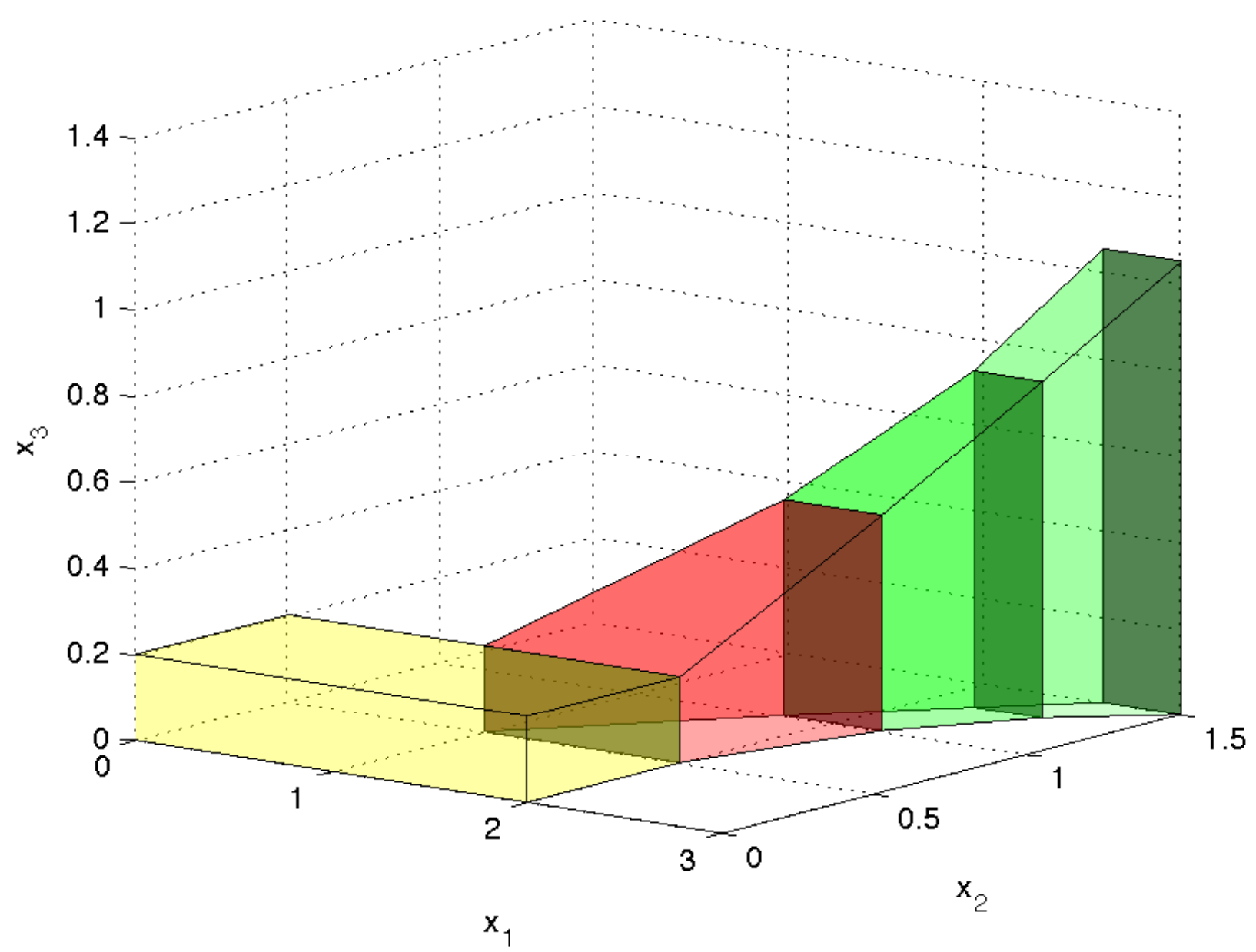

Figure 8.16: State-space areas satisfying constraints for modes/events of control specification (Case 2) 


\begin{tabular}{|c|c|c|c|c|}
\hline \multicolumn{5}{|c|}{ Simplex Data } \\
\hline $\mathcal{S}_{k}$ & $v_{0}$ & $v_{1}$ & $v_{2}$ & $v_{3}$ \\
\hline $\mathcal{S}_{1}$ & $(0,0,0)$ & $(1,0,0)$ & $(1,0.5,0.2)$ & $(1,0.5,0)$ \\
\hline $\mathcal{S}_{2}$ & $(1,0,0)$ & $(2,0.5,0)$ & $(1,0.5,0.2)$ & $(1,0.5,0)$ \\
\hline $\mathcal{S}_{3}$ & $(1,0.5,0)$ & $(1,0.5,0.2)$ & $(1.75,1,0)$ & $(2,0.5,0)$ \\
\hline $\mathcal{S}_{4}$ & $(1,0.5,0.2)$ & $(1.75,1,0.5)$ & $(1.75,1,0)$ & $(2,0.5,0)$ \\
\hline $\mathcal{S}_{5}$ & $(2,0.5,0)$ & $(2.25,1,0)$ & $(1.75,1,0.5)$ & $(1.75,1,0)$ \\
\hline $\mathcal{S}_{6}$ & $(1.75,1,0)$ & $(1.75,1,0.5)$ & $(2.25,1.3,0)$ & $(2.25,1,0)$ \\
\hline $\mathcal{S}_{7}$ & $(1.75,1,0.5)$ & $(2.25,1.3,0.75)$ & $(2.25,1.3,0)$ & $(2.25,1,0)$ \\
\hline $\mathcal{S}_{8}$ & $(2.25,1,0)$ & $(2.6,1.3,0)$ & $(2.25,1.3,0.75)$ & $(2.25,1.3,0)$ \\
\hline $\mathcal{S}_{9}$ & $(2.25,1.3,0)$ & $(2.25,1.3,0.75)$ & $(2.6,1.5,0.1)$ & $(2.6,1.3,0)$ \\
\hline $\mathcal{S}_{10}$ & $(2.25,1.3,0.75)$ & $(2.6,1.5,0.9)$ & $(2.6,1.5,0.1)$ & $(2.6,1.3,0)$ \\
\hline $\mathcal{S}_{11}$ & $(2.6,1.3,0)$ & $(2.6,1.5,0.9)$ & $(2.6,1.5,0.1)$ & $(3,1.5,0.1)$ \\
\hline
\end{tabular}

Table 8.2: Simplex data (Case 2) 
RCP. The resulting piecewise affine feedback law is as follows:

$$
\begin{array}{lll}
u & =\left[\begin{array}{lll}
0 & 19.8 & 0
\end{array}\right] x+0.1, & x \in \mathcal{S}_{1} \\
u & =\left[\begin{array}{lll}
-2 & 4 & -42.5
\end{array}\right] x+12 & x \in \mathcal{S}_{2} \\
u & =\left[\begin{array}{lll}
-3 & 8.5 & 10
\end{array}\right] x+8.75, & x \in \mathcal{S}_{3} \\
u & =\left[\begin{array}{lll}
-1 & 7.5 & -10
\end{array}\right] x+8.25, & x \in \mathcal{S}_{4} \\
u & =\left[\begin{array}{lll}
-14 & -3 & -22
\end{array}\right] x+42.5, & x \in \mathcal{S}_{5} \\
u & =\left[\begin{array}{lll}
-6 & 3.333 & 0
\end{array}\right] x+19.1667, & x \in \mathcal{S}_{6} \\
u & =\left[\begin{array}{lll}
1 & 6.666 & 0
\end{array}\right] x+1.083, & x \in \mathcal{S}_{7} \\
u & =\left[\begin{array}{lll}
-14.2857 & 6.667 & -9.333
\end{array}\right] x+38.47 & x \in \mathcal{S}_{8} \\
u & =\left[\begin{array}{lll}
-5.714 & 9.333 & 1.333
\end{array}\right] x+12.72, & x \in \mathcal{S}_{9} \\
u & =\left[\begin{array}{lll}
8.48 & -5.3125 & 0.625
\end{array}\right] x-0.147, & x \in \mathcal{S}_{10} \\
u & =\left[\begin{array}{lll}
2.5 & 7.5 & 0
\end{array}\right] x-3.75 & x \in \mathcal{S}_{11}
\end{array}
$$

Figure 8.17 shows the state trajectory through the triangulated space. From Figure 8.18 which shows the BIS variation with time, it is clear that the controller decreases the BIS level smoothly and the patient is transfered from fully conscious to Stage 3 plane III. In addition, the piecewise affine feedback law defined over the 11 simplices simultaneously ensures that both the inspired drug concentration as well as the concentration in the peripheral compartment evolve in the correct manner during the transient period between full consciousness and the stage whereby the patient is ready for surgery .

The examples have shown how complex or unconventional control requirements requiring a high degree of expressivity in the specification terminology are achievable using a triangulation of the state space resulting in series of reach problems formulated on simplices solved by a piecewise affine feedback. The deep study into solution of the 


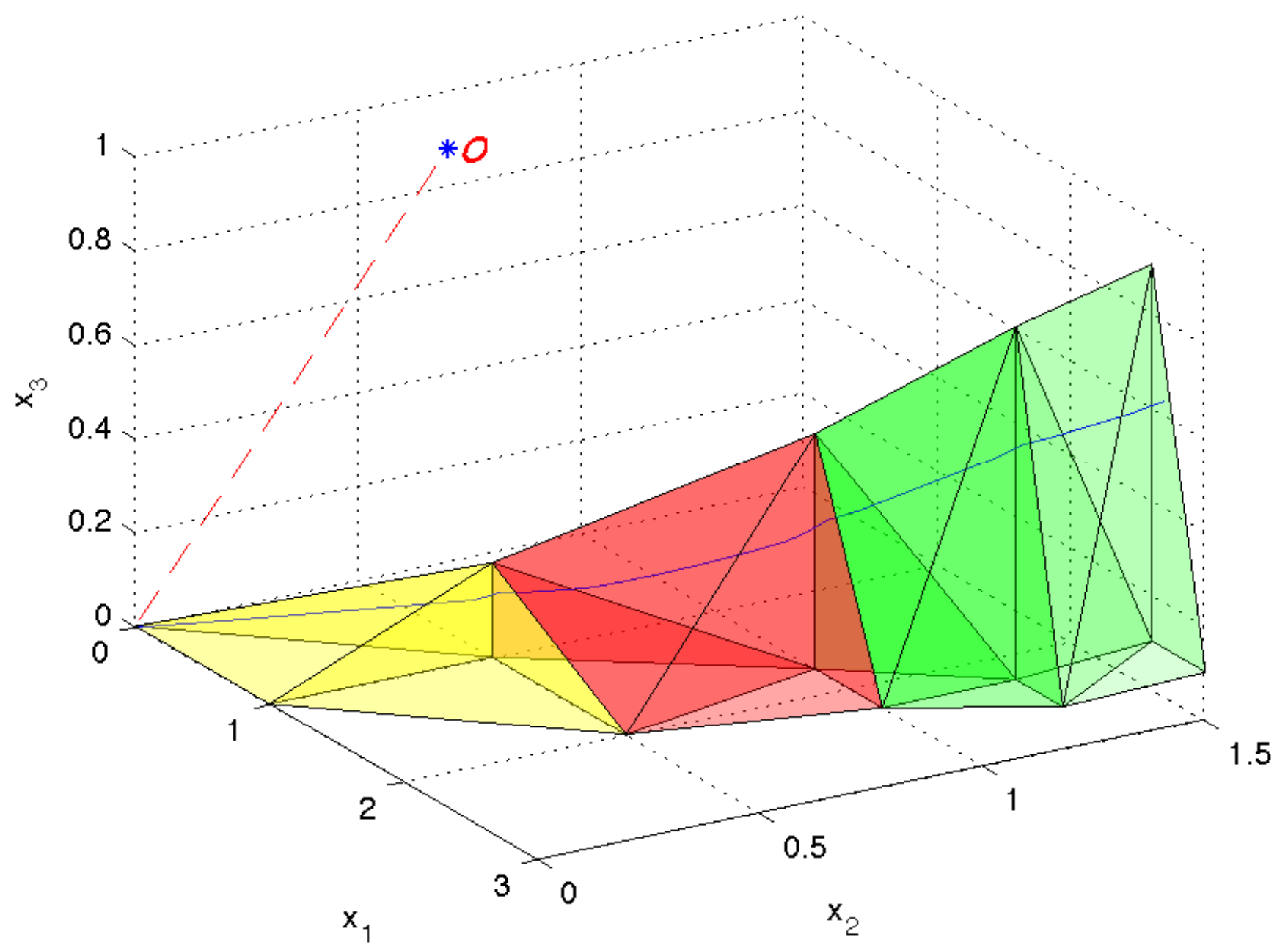

Figure 8.17: Triangulated state-space showing state trajectory under piecewise affine controller: Case 2 


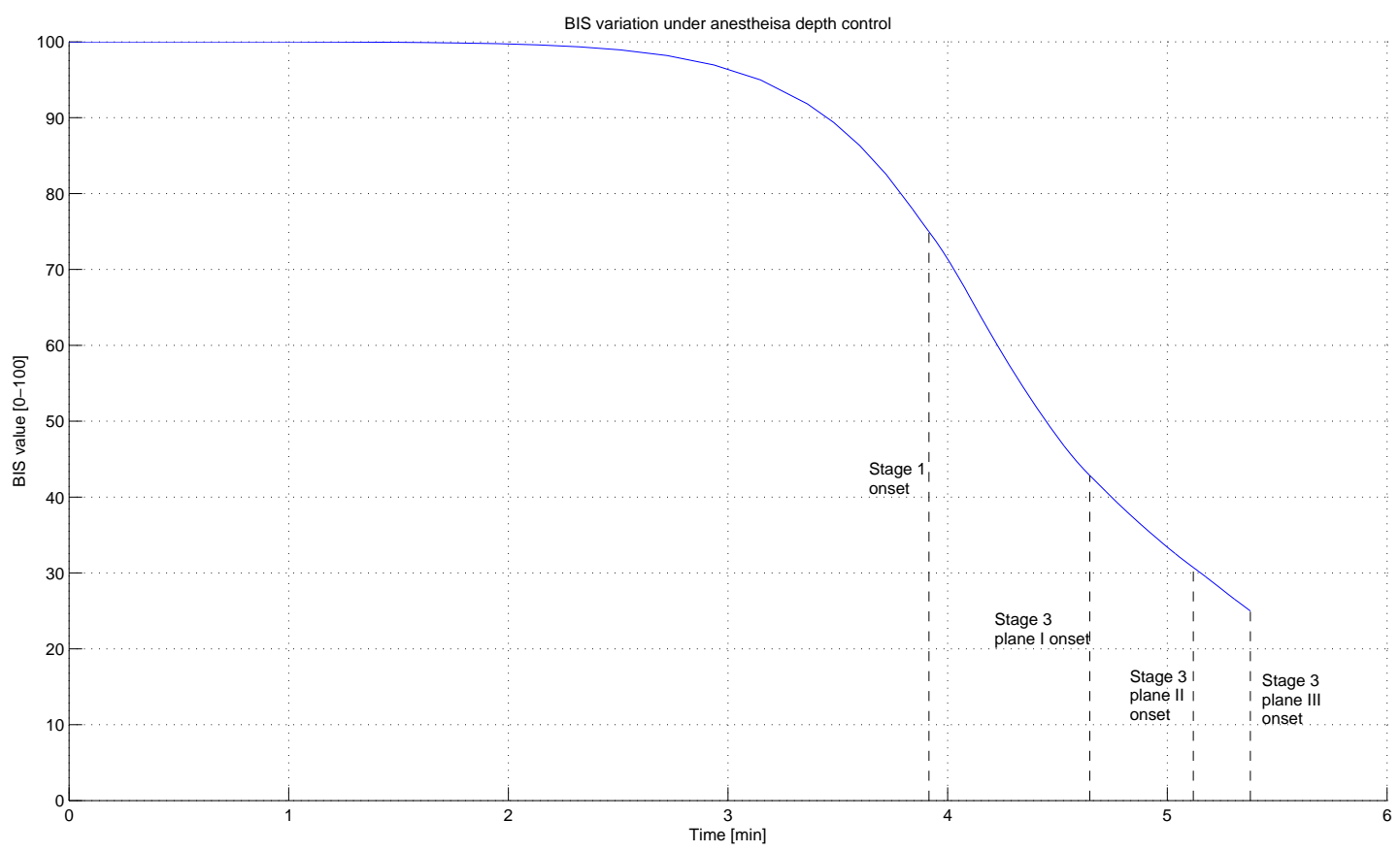

Figure 8.18: BIS variation under piecewise affine controller: Case 2

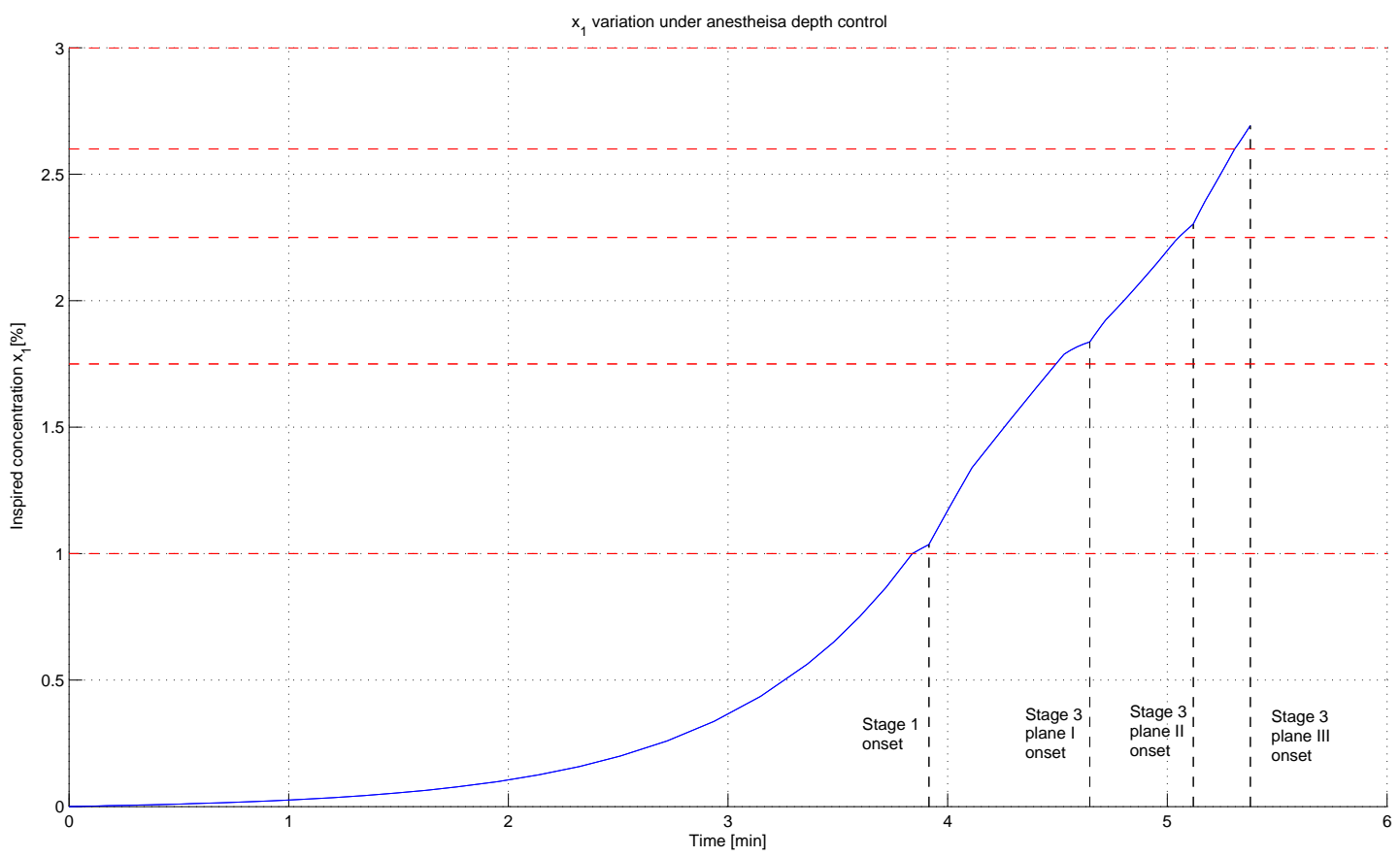

Figure 8.19: Inspired drug concentration under piecewise affine controller: Case 2 


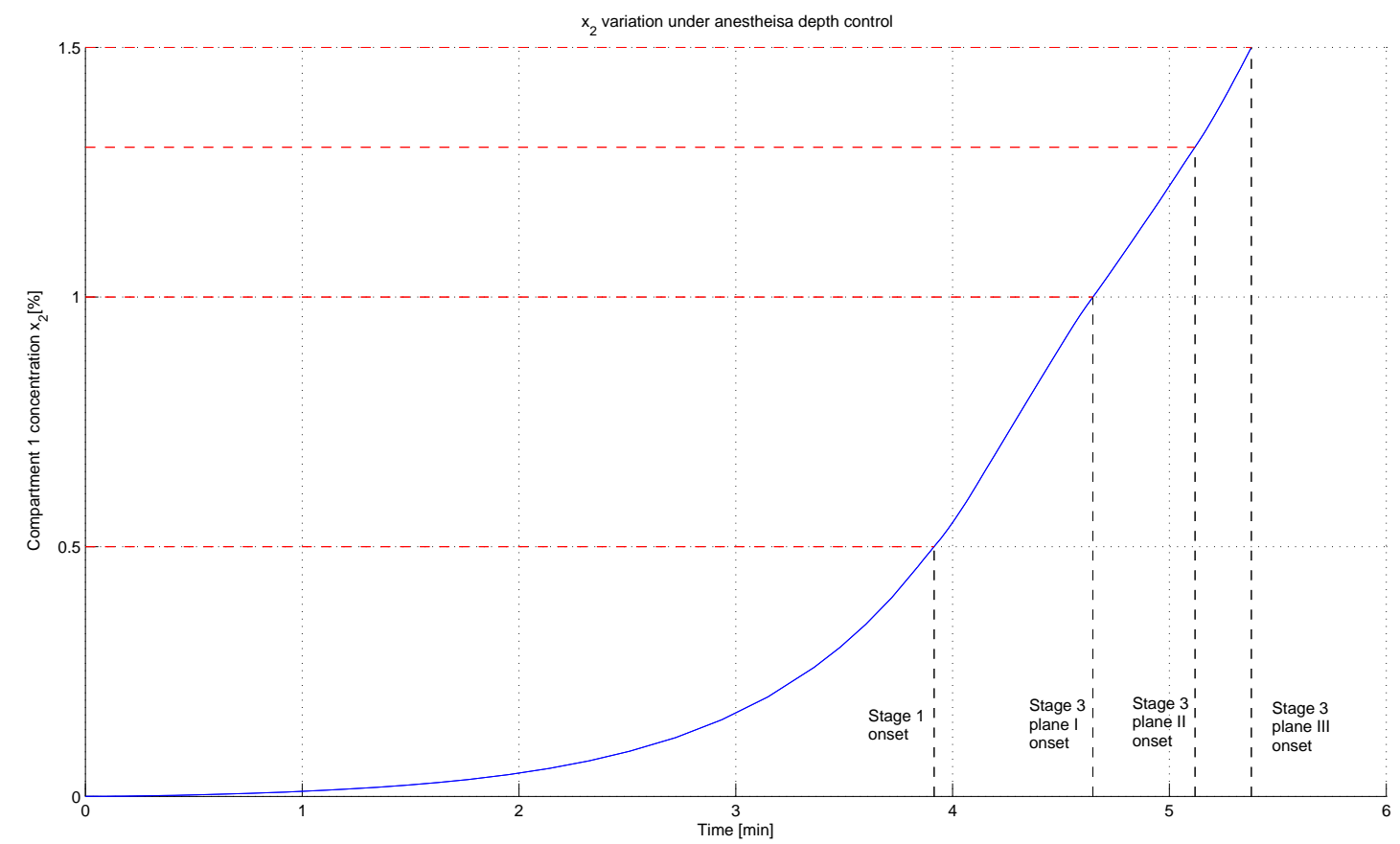

Figure 8.20: Central compartment drug concentration under piecewise affine controller:

Case 2 


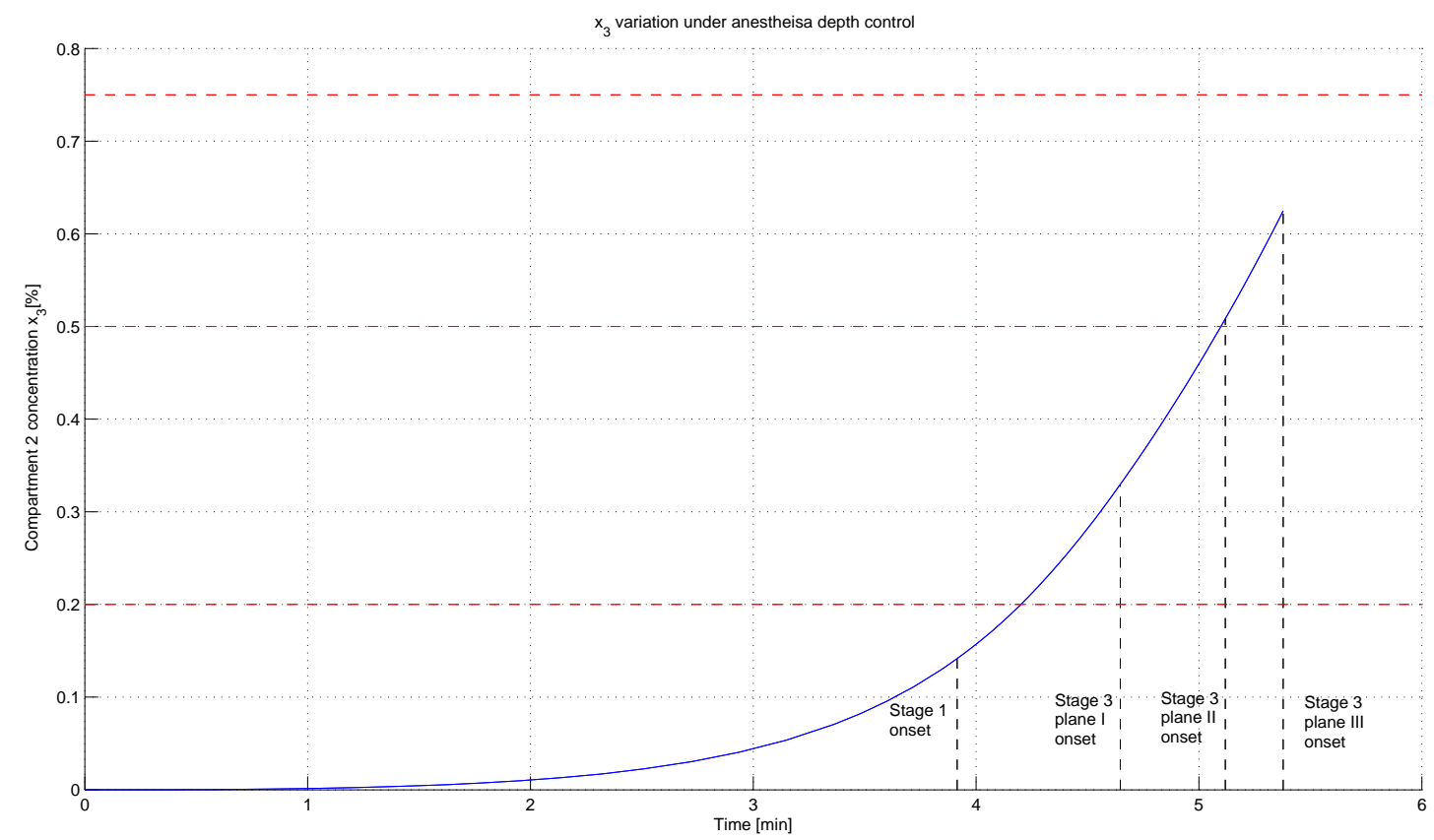

Figure 8.21: Second compartment drug concentration under piecewise affine controller: Case 2

$\mathrm{RCP}$ presented in the thesis is therefore an important feature in such engineering design processes. 


\section{Chapter 9}

\section{Conclusions}

The thesis presents a complete solution to the reach control problem for affine systems on simplices. The objective is to find a control assignment which ensures that all closedloop trajectories with initial conditions in a simplex $\mathcal{S}$ reach and exit a target facet $\mathcal{F}_{0}$, without previously leaving $\mathcal{S}$. The main features of the problem and the solution presented are derived from the geometry of the simplex as well the convexity properties of affine dynamics. We review the existing results for synthesis of an affine feedback law $u(x)=K x+g$. We review a first set of necessary and sufficient conditions for solvability of RCP by affine feedback. These conditions consist of the invariance conditions and a flow condition.

It is computationally difficult to construct an affine law by simultaneously solving the invariance conditions and flow condition. In addition, these necessary and sufficient conditions provide no information into the structural aspects of the system with respect to the simplex which affect solvability of RCP. We therefore examine the problem under a specific assumption on the triangulation of the state-space and present geometric sufficient conditions for solvability by affine feedback. These newer sufficient conditions are verifiable geometric properties of the system which arise from the interaction of simplex and affine dynamics and effectively replace the flow condition. 
It is shown that affine feedback is equivalent to continuous state feedback in terms of solvability of RCP, and that the failure of continuous state feedback to solve the problem is due to the appearance of closed-loop equilibria in $\mathcal{S}$. In such cases, the reach control indices can isolate equilibria and describe the system structural properties which contribute to their formation.

A subdivision algorithm based on the reach control indices is proposed to perform triangulation of the simplex and to obtain a piecewise affine feedback solution for those cases where continuous state feedback fails. It is proven that RCP is solvable by piecewise affine feedback provided it is solvable by open loop controls. Examples illustrating RCP and its solution using both continuous and piecewise affine feedback are included in the thesis to reinforce the theory developed .

As demonstrated using a biomedical engineering application, the design and implementation of complex control specifications can be achieved by performing triangulation of the state-space in conjunction with a piecewise affine control. Such a design approach gives rise to a series of reach control problems formulated on simplices and hence a rigorous study on the solution of RCP as provided in this thesis is therefore essential to the development of this novel control paradigm. 


\section{Bibliography}

[1] M. Baric, P. Grieder, M. Baotic, and M. Morari. An efficient algorithm for optimal control of PWA systems with polyhedral performance indices Automatica. vol. 44, Issue 1, January 2008, pp. 296-301.

[2] A. Bemporad, G. Ferrari-Trecate, and M. Morari. Observability and Controllability of Piecewise Affine and Hybrid Systems. IEEE Transactions on Automatic Control. vol. 45, no. 10, October 2000.

[3] A. Bemporad and M. Morari. Control of systems integrating logic, dynamics, and constraints. Automatica. vol. 35, pp. 407-428, March 1999.

[4] A. Berman and R.J. Plemmons. Nonnegative Matrices in the Mathematical Sciences. Academic Press, New York. 1979.

[5] F. Blanchini and S. Miani. Set-Theoretic Methods in Control. Birkhauser, 2008.

[6] F. Blanchini, A. Megretski. Robust state feedback control of LTV systems: nonlinear is better than linear. IEEE Trans Automatic Control. vol 44, issue 4, April 1999, pp. $802-807$.

[7] F. Blanchini and F.A. Pellegrino. Relatively optimal control: A static piecewise affine solution. SIAM Journal on Control and Optimization vol. 46, issue 2, p585603, 2007. 
[8] K. Border. Fixed Point Theorems with Applications to Economics and Game Theory. Cambridge University Press, 1985.

[9] M.E. Broucke. Reach control on simplices by continuous state feedback. SIAM Journal on Control and Optimization. vol. 48, issue 5, pp. 3482-3500, February 2010.

[10] M.K. Camlibel, W.P.M.H. Heemels, and J.M. Schumacher. A full characterization of stabilizability of bimodal piecewise linear systems with scalar inputs. Automatica. 44(5):1261-1267, 2008.

[11] F. Clarke, Y. Ledyaev, R. Stern, and P. Wolenski. Nonsmooth Analysis and Control Theory. Springer-Verlag, New-York, 1998.

[12] F.H. Clarke, Yu.S. Ledyaev, E.D. Sontag, and A.I. Subbotin. Asymptotic controllability implies feedback stabilization. IEEE Trans. Automat. Control. 42 (1997), pp. 1394-1407.

[13] P.J. Collins, L.C.G.J.M. Habets, A. Kuut, M. Nool, M. Petreczky, and J.H. van Schuppen. ConPAHS - A software package for control of piecewise-affine hybrid systems. Proc. 2006 IEEE Conference on Computer Aided Control System Design (CACSD '06). (Munich, Germany, October 4-6, 2006), IEEE Press, New York, pp. $76-81,2006$.

[14] L.C.G.J.M. Habets and J.H. van Schuppen. Control of piecewise-linear hybrid systems on simplices and rectangles, in: M.D. Di Benedetto and A.L. SangiovanniVincentelli (Eds.) Hybrid Systems: Computation and Control, Lecture Notes in Computer Science. Springer Verlag, vol. 2034, pp. 261-274, 2001.

[15] L.C.G.J.M. Habets and J.H. van Schuppen. A control problem for affine dynamical systems on a full-dimensional polytope. Automatica. no. 40, pp. 21-35, 2004. 
[16] L.C.G.J.M. Habets, P.J. Collins, and J.H. van Schuppen. Reachability and control synthesis for piecewise-affine hybrid systems on simplices. IEEE Trans. Automatic Control. no. 51, pp. 938-948, 2006.

[17] J. Hudson. Piecewise Linear Topology. University of Chicago Lecture Notes. W.A. Benjamin Inc. 1969.

[18] C. W. Lee. Subdivisions and triangulations of polytopes. Handbook of Discrete and Computational Geometry. CRC Press Series Discrete Math. Appl., pp. 271-290, 1997.

[19] Z. Lin and M.E. Broucke. Resolving control to facet problems for affine hypersurface systems on simplices. IEEE Conference on Decision and Control (CDC '06). December 2006.

[20] Z. Lin and M.E. Broucke. Reachability and control of affine hypersurface systems on polytopes. IEEE Conference on Decision and Control (CDC '07). December 2007.

[21] R.T Rockafellar. Convex Analysis. Princeton University,Princeton, New Jersey 1970.

[22] B. Roszak and M. E. Broucke. Necessary and sufficient conditions for reachability on a simplex. Automatica. vol. 42, no. 11, pp. 1913-1918, November 2006.

[23] Y.A. Shashkin. Fixed Points. American Mathematical Society, Providence, R.I., 1991.

[24] O. Toker and H. Özbay. On the $\mathcal{N} P$-hardness of solving bilinear matrix inequalities and simultaneous stabilization with static output feedback. Proceedings of the 1995 American Control Conference, Seattle, Washington, 1995.

[25] M. L. Tyler and M. Morari. Propositional logic in control and monitoring problems. Automatica, vol. 35, no. 4, pp. 565-582, 1999. 
[26] W. Murray Wonham. Linear Multivariable Control: a Geometric Approach. 3rd Ed. Springer-Verlag, 1985.

[27] G. M. Zeigler. Lectures on Polytopes. Graduate Texts in Mathematics. Springer, 1995.

[28] C. Beck, HH Lin and M. Bloom Modeling and Control of Anesthetic Pharmacodynamics, in : Biology and Control Theory: Current Challenges Lecture Notes in Control and Information Sciences Springer Berlin/Heidelberg, vol. 357, pp. 263-289, 2007

[29] Bibian S., Ries, C., Huzmezan, M., Dumont, G. Clinical Anesthesia and Control Engineering:Terminology, Concepts and Issues European Control Conference 2003

[30] C. Frei, A. Gentilini, M. Rossoni-Gerosai, R. Wymann, M. Morari, T. Schnider, and A. Zbinder Modeling and Closed-loop control of hypnosis by means of BIS with Isoflurane IEEE Transactions on Biomedical Engineering, vol.48, No. 8, August 2001.

[31] A.L. Gentilini Feedback Control of Hypnosis and Analgesia in Humans Phd Dissertation, ETH, 2001

[32] J. Bailey, W. Haddad, J. Im, T. Hayakawa, P. Nagel Adaptive and Neural Network Adaptive Control of depth of anesthesia during surgery American Control Conference, June 14-16, 2006

[33] A.K. Bhargava, R. Setlur, and D. Sreevastava Correlation of Bispectral index and Guedel's Stages of Ether Anesthesia Anesthesia and Analgesia, vol. 98, pp 132-134, 2004 